\title{
Ein Gebietszerlegungsverfahren für parabolische Probleme im Zusammenhang mit Finite-Volumen-Diskretisierung
}

\author{
Dissertation \\ zur Erlangung des Doktorgrades \\ der Mathematisch-Naturwissenschaftlichen Fakultäten \\ der Georg-August-Universität zu Göttingen
}

vorgelegt von

Joachim Thaddäus Held

aus Göttingen

Göttingen 2006 
D7

Referent: Prof. Dr. G. Lube

Korreferent: Prof. Dr. R. Schaback

Tag der mündlichen Prüfung: 21.12.2006 


\section{Inhaltsverzeichnis}

$\begin{array}{lc}\text { Einleitung } & 3\end{array}$

\begin{tabular}{llr}
\hline I & Finite-Volumen-Verfahren & 11
\end{tabular}

1 Zeitabhängige Konvektions-Diffusions-Probleme 13

1.1 Kontinuierliches Problem . . . . . . . . . . . . . . . . . 13

1.2 Semidiskretes Problem . . . . . . . . . . . . . . . . . . 20

2 Finite-Volumen-Verfahren $\quad 23$

2.1 Ausgangstriangulierung . . . . . . . . . . . . . . . . . 23

2.2 Finite-Volumen-Räume . . . . . . . . . . . . . . . . . . . . 25

2.3 Finite-Volumen-Diskretisierung . . . . . . . . . . . . . . . 27

2.4 Gleichgewichts- und Regularitätsbedingungen . . . . . . . . . . . . . . 29

2.5 Konstruktion von Donald-Netzen . . . . . . . . . . . . . . . . . 31

3 Fehlerabschätzung zum Finite-Volumen-Verfahren 35

3.1 Konsistenzfehlerabschätzung . . . . . . . . . . . . . . . . 35

3.2 Eindeutige Lösbarkeit und Stabilitätsabschätzungen . . . . . . . . . . . . . 39

3.3 A-priori-Fehlerabschätzungen zum semidiskreten Problem . . . . . . . . . . 41

3.4 A-priori-Fehlerabschätzungen zum volldiskreten Problem . . . . . . . . . . 50

4 Stabilisierte Finite-Volumen-Verfahren $\quad 59$

4.1 Upwind-Modifikation . . . . . . . . . . . . . . . . . . 59

4.2 Stabilität . . . . . . . . . . . . . . . . . . 64

4.3 Konsistenzfehlerabschätzung . . . . . . . . . . . . . . . 66 
4.4 A-priori-Fehlerabschätzung . . . . . . . . . . . . . . . . . 69

\begin{tabular}{lll}
\hline II Gebietszerlegungsverfahren & 71
\end{tabular}

5 Gebietszerlegungsverfahren für parabolische Probleme $\quad 73$

5.1 Zweigebietsformulierung . . . . . . . . . . . . . . 73

5.2 Ein Dirichlet-Robin-Algorithmus . . . . . . . . . . . . . 77

6 Konvergenzanalyse eines Dirichlet-Robin-Verfahrens 85

6.1 Fehlergleichungen . . . . . . . . . . . . . . . . . 85

6.2 Steklov-Poincaré-Operatoren für parabolische Probleme . . . . . . . . . 88

6.3 Ein Konvergenzresultat . . . . . . . . . . . . . . . . . . . . 91

7 Gebietszerlegung und Finite-Volumen-Diskretisierung 99

7.1 Finite-Volumen-Approximation . . . . . . . . . . . . . . . 99

7.2 Der Dirichlet-Robin-Algorithmus für das semidiskretisierte Problem . . . . 103

7.3 Konvergenzanalyse im semidiskretisierten Fall . . . . . . . . . . . . . . 106

7.4 Numerische Ergebnisse . . . . . . . . . . . . . . . . . . . . 110

8 Optimale Austauschrandbedingungen $\quad 125$

8.1 Untersuchung des Dirichlet-Robin-Algorithmus im eindimensionalen Fall . . . 125

8.2 Untersuchung des Dirichlet-Robin-Algorithmus im zweidimensionalen Fall . . 131

8.3 Numerische Ergebnisse . . . . . . . . . . . . . . . . . . . . . . 133

8.4 Ausblick . . . . . . . . . . . . . . . . . . . . . 142

A Anhang $\quad 145$

A.1 Notation . . . . . . . . . . . . . . . . . . . 145

A.2 Funktionenräume ortsabhängiger Funktionen . . . . . . . . . . . . 146

A.3 Funktionenräume zeitabhängiger Funktionen . . . . . . . . . . . . . 151

$\begin{array}{ll}\text { Literaturverzeichnis } & 155\end{array}$ 


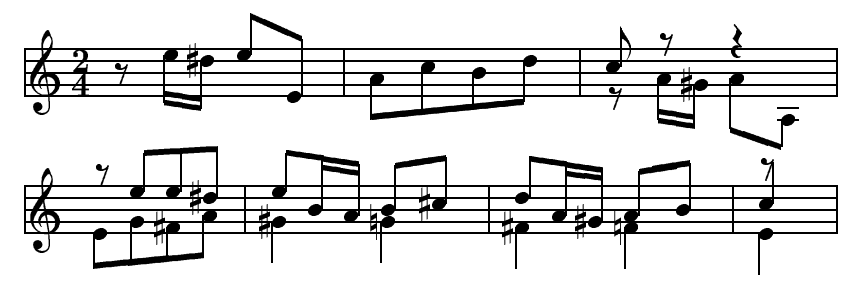

J. S. BACH, Sonata für Violine solo No. 2 a-moll, Fuga

\section{Einleitung}

Den Gegenstand dieser Arbeit bilden ein Finite-Volumen-Verfahren und ein iteratives Gebietszerlegungsverfahren für instationäre Konvektions-Diffusions-Reaktions-Gleichungen der Form

$$
\partial_{t} u-\nabla \cdot(A \nabla u-b u)+c u=f .
$$

In der Strömungsmechanik beschreiben Konvektions-Diffusions-Gleichungen die Verteilung einer physikalischen Größe (etwa Temperatur, Stoffkonzentration, Impuls) unter dem Einfluß eines ortsabhängigen Strömungsfeldes (Konvektion), mikroskopischer Teilchenbewegung (Diffusion) und möglicherweise vorhandener äußerer Quellen. Sie treten unter stark vereinfachenden Annahmen als Teilprobleme der Navier-Stokes-Gleichungen auf (Modellgleichungen, siehe Kapitel 7 in [38]) und weisen im Fall sehr kleiner Diffusion gegenüber der Stärke der Konvektion typischerweise Grenzschichtphänomene auf.

Die vorliegende Arbeit entstand während meiner Tätigkeit am Institut für Aerodynamik und Strömungstechnik des Deutschen Zentrums für Luft- und Raumfahrt. Dort wurden FiniteVolumen-Verfahren zu hoher Anwendungsreife gebracht und bewähren sich mit großem Erfolg. Den Strömungsproblemen liegen in der Regel sehr große Rechengebiete zugrunge, die auf eine sehr große Zahl von Unbekannten führen und die Regionen mit äußerst unterschiedlichen Anforderungen an die Diskretisierung aufweisen. Vor diesem Hintergrund ergibt sich die Themenstellung der Arbeit.

\section{Finite-Volumen-Verfahren}

Finite-Volumen-Verfahren bilden eine Klasse von Diskretisierungsschemata, die sich als sehr geeignet erwiesen haben bei der numerischen Behandlung partieller Differentialgleichungen, die in Divergenzform vorliegen oder zumindest einen Divergenzterm enthalten. Insbesondere eignen sie sich zur Diskretisierung von physikalischen Erhaltungssätzen

$$
\partial_{t} u-\nabla \cdot F(u)=0
$$

mit einem im allgemeinen nichtlinear von $u$ abhängigen Vektorfeld $F$. Damit sind FiniteVolumen-Verfahren in der numerischen Strömungsberechnung von besonderem Interesse und wurden erstmals 1971 zur Lösung der zeitabhängigen zweidimensionalen Euler-Gleichungen eingesetzt $([51,50])$.

Die Finite-Volumen-Diskretisierung baut auf einer Zerlegung des Rechengebietes in endlich viele polygonal berandete, paarweise disjunkte Teilgebiete, die sogenannten Kontrollvolumina 
oder Boxen, auf. Bei der Wahl der Kontrollvolumenform hat man dabei prinzipiell wenig Einschränkungen, woraus sich eine hohe Flexibilität der Diskretisierung ergibt. Abhängig von der Lokalisierung der Unbekannten bezüglich der Kontrollvolumina spricht man von cell-centeredund cell-vertex-Verfahren. Im ersten Fall werden die Unbekannten dem Kontrollvolumen zugeordnet, im zweiten Fall werden sie in den Eckpunkten lokalisiert. Im nächsten Schritt werden die Problemgleichungen über jedem dieser Kontrollvolumina integriert. Dies entspricht einer Variationsformulierung mit bezüglich der Kontrollvolumina konstanten Testfunktionen. Die Divergenzterme der Gleichung werden über jedem Kontrollvolumen in Oberflächenintegrale überführt, die dann auf sehr unterschiedliche Weise approximiert werden können. Eine umfassende Darstellung der Finite-Volumen-Technik bietet [21], eine detaillierte Untersuchung zur Anwendung auf Konvektions-Diffusions-Gleichungen findet man in [41]. Verallgemeierungen von Finite-Volumen-Methoden zu Verfahren höherer Ordnung werden in [8] betrachtet. Zum Einsatz von Finite-Volumen-Methoden speziell in der Strömungsmechanik siehe [38].

Ein enger Zusammenhang zwischen Finite-Volumen- und Finite-Element-Verfahren ergibt sich, wenn man die Kontrollvolumina dual zu einer üblichen Finite-Element-Triangulierung bestimmt, indem man jedem Knoten der Triangulierung genau ein Kontrollvolumen zuordnet. Das Mengesystem der Kontrollvolumina heißt dann duales oder auch sekundäres Boxgitter. Der damit verbundene Isomorphismus zwischen den entsprechenden Ansatzfunktionen erlaubt die Formulierung der Finite-Volumen-Diskretisierung als einen konformen verallgemeinerten Galerkin-Ansatz, einer Galerkin-Methode also, bei dem sich Linear- und Bilinearformen der Diskretisierung von denen des kontinuierlichen Problems unterscheiden. Bei dieser sogenannten Finite-Volumen-Finite-Element-Methode greift die Konvergenztheorie der klassischen Finite-Volumen-Technik nicht (vergl. Abschnitt 3.4.3 in [21]). Jedoch steht durch die Arbeiten [7, 35, 9] im Fall von stationären Konvektions-Diffusions-Reaktions-Problemen eine vollständige (spezifische) Konvergenztheorie zur Verfügung. Wie die genannten Arbeiten darlegen, ergeben sich unter gewissen Gleichgewichtsbedingungen an die Kontrollvolumina im wesentlichen die gleichen Fehlerabschätzungen wie bei einer Finite-Element-Diskretisierung mit stückweise affinen Ansatzfunktionen, für reine Diffusionsprobleme sogar identische Diskretisierungen.

Neben der besonderen Eignung für die Diskretisierung von Erhaltungsproblemen zeichnet Finite-Volumen-Verfahren ein weiterer Aspekt aus. Mit ihnen existieren eine ganze Reihe von finite-volumen-spezifischen Upwind-Schemata zur Stabilisierung in konvektionsdominanten Fällen (siehe etwa [3, 43]), darunter monotonieerhaltende Verfahren (die von erster Ordnung sind). Diese Stabilisierungen beeinflussen die Lösung hauptsächlich in Konvektionsrichtung (Stromlinie), allerdings treten auch quer dazu Effekte auf, die die Lösung "verschmieren“. In [5] wird ein Finite-Volumen-Upwind-Schema vorgestellt, bei dem diese Quereffekte reduziert werden. Man kann also die Finite-Volumen-Finite-Element-Methode als Möglichkeit betrachten, Upwind-Techniken im Finite-Element-Kontext einzusetzen. Daneben lassen sich Finite-Volumen-Verfahren auch mit Stromlinien-Diffusions-Methoden stabilisieren. Numerische Resultate, die auf diese Weise gewonnen werden, sind gleichwertig zu Resultaten, die mit Finite-Element-Verfahren und derselben Stabilisierung produziert werden ([60]).

Ein Schwerpunkt dieser Arbeit ist, das erwähnte, von einer Finite-Element-Triangulierung ausgehende Finite-Volumen-Verfahren auf zeitabhängige Konvektions-Diffusions-ReaktionsProbleme zu erweitern. Vervollständigt wird dies durch die Konstruktion einer UpwindModifikation. Dabei wird im dieser Arbeit die erwähnte Konvergenztheorie auf parabolische 
Probleme, inklusive konvektionsdominierter Fälle, ausgedehnt. Zudem wird damit die Voraussetzung für die Anwendung der Finite-Volumen-Diskretisierung im Zusammenhang mit einem Gebietszerlegungsverfahren vom Waveform-Relaxation-Typ geschaffen, wie es im zweiten Teil entwickelt wird.

\section{Gebietszerlegungsverfahren}

Das Prinzip von Gebietszerlegungsverfahren ist, das ursprüngliche Rechengebiet eines Randwert- oder Anfangsrandwertproblems vollständig in Teilgebiete aufzuteilen und die Problemgleichungen über jedem dieser Teilgebiete zu lösen. Die Kopplung der so entstehenden Teilprobleme geschieht dabei allein durch Transmissionsbedingungen an den inneren Teilgebietsrändern, dem Austauschrand. Zwei Aspekte bei der Diskretisierung von partiellen Differentialgleichungen machen den Einsatz von Gebietszerlegenungsverfahren wegen der Betrachtung lokaler Probleme im besonderen Maße attraktiv. Zum einen kann eine sehr große Anzahl von Unbekannten die Parallelisierung der Rechnung nötig machen oder eine Parallelisierung wird angestrebt, um Performance-Gewinn zu erzielen. Zum anderen können unterschiedliche physikalische Bedingungen zu unterschiedlichen Problemgleichungen führen oder den Bedarf unterschiedlicher Diskretisierungsmethoden innerhalb eines Rechengebiets erzeugen.

Je nachdem, ob die Teilgebiete paarweise disjunkt sind oder nicht, spricht man von nicht überlappenden bzw. überlappenden Verfahren. Die ersten Anwendungen von Gebietszerlegungsverfahren nutzten überlappende Teilgebiete sowie iterativ Dirichlet-Austauschrandbedingungen. Sie gehen zurück auf die Arbeit von H. A. Schwarz, der bereits 1869 diese Methode einsetzte, um zu einem Existenzresultat für das Poisson-Problem über Gebieten mit nicht glatten Rändern zu gelangen. Es hat sich daher auch die Bezeichnung Schwarz-Methode durchgesetzt (zur Geschichte der Gebietszerlegungsmethoden siehe [64]; eine umfassende Darstellung zu etablierten Techniken findet man in den Monographien [58] und [62]). Vor allem jedoch, wenn die Zerlegung des Rechengebietes durch unterschiedliche physikalische Bedingungen oder Diskretisierungsansprüche motiviert ist, sind disjunkte Teilgebiete im Vorteil. Verfahren, die iterativ die Gleichungen über jedem Teilgebiet lösen bei Vorgabe von Randbedingungen, die sich aus den jeweils benachbarten Teilgebietslösungen des vorangegangenen Iterationsschrittes ergeben, heißen Teilgebiets-Iterationstechniken (in der Literatur iteration-by-subdomain methods). Mit Hilfe von bestimmten Operatoren, die für Funktionen über dem Austauschrand definiert sind, genannt Steklov-Poincaré-Operatoren, lassen sich isoliert Iterationsgleichungen für die Randwerte der Teilgebietslösungen formulieren (Schur-Komplement-Gleichungen genannt). Diese Operatoren können als wesentliches Mittel zur Konvergenzanalyse herangezogen werden.

Bei der klassischen Anwendung von Gebietszerlegungsverfahren auf Evolutionsprobleme wird in einem ersten Schritt implizit in der Zeit diskretisiert, um dann in jedem Zeitschritt einen Gebietszerlegungsalgorithmus auf das entstandene stationäre Problem anzuwenden. Beispielsweise findet man in [13, 14] die Anwendung klassischer Schwarz-Methoden, in [18] die Anwendung eines Dirichlet-Neumann-Algorithmus. Ein Robin-Robin-Algorithmus wird in [46] untersucht (bei Zeitdiskretisierung durch ein DG-Verfahren). Diese Reihenfolge von Zeitdiskretisierung und Gebietszerlegung bringt jedoch einen deutlichen Nachteil bei der Zeitdiskretisierung mit sich, denn allen Teilproblemen liegt dieselbe Zeitschrittweite zugrunde. Ein weiterer Nachteil ergibt sich aus der Notwendigkeit, Randwerte am Austauschrand in jedem Zeitschritt auszutauschen. 
Einleitung

In der voliegenden Arbeit wird ein anderer Weg eingeschlagen. Wir gehen im Sinne der Waveform-Relaxation-Methode vor, ein Verfahren, das ursprünglich zur Lösung extrem großer Systeme gewöhnlicher Differentialgleichungen in der Zeit Anwendung fand (siehe [44, 53]). Man beginnt mit der Zerlegung des Rechengebiets und formuliert den Gebietszerlegungsalgorithmus für das zeitkontinuierliche Problem. Über jedem der Teilgebiete wird somit wiederum ein Evolutionsproblem gelöst. Ausgehend von einem Initialwert $u_{1}^{0}$ entsteht für Probleme der Form

$$
\partial_{t} u+L u=f \text { in }(0, T) \times \Omega
$$

mit einem räumlichen Differentialoperator $L$ sowie Anfangs- und Randbedingung auf $(0, T) \times$ $\partial \Omega$ im Fall eines iterativen Gebietszerlegungsalgorithmus hier vereinfachend für zwei Teilgebiete $\Omega_{1}, \Omega_{2}$ das folgende, schematisch dargestellte Verfahren: Für $k=1,2, \ldots$ löse

$$
\left\{\begin{array}{l}
\partial_{t} u_{1}^{k}+L u_{1}^{k}=f \text { in }(0, T) \times \Omega_{1} \text {, ergänzt durch } \\
\text { Anfangs- und Randbedingung auf }(0, T) \times \partial \Omega_{1} \cap \partial \Omega \text { für } u_{1}^{k} \text { sowie } \\
\text { Austauschrandbedingung } \Phi_{1}\left(u_{1}^{k}\right)=\Psi_{1}\left(u_{1}^{k-1}, u_{2}^{k-1}\right) \text { auf }(0, T) \times \partial \Omega_{1} \cap \partial \Omega_{2},
\end{array}\right.
$$

$$
\left\{\begin{array}{l}
\partial_{t} u_{2}^{k}+L u_{2}^{k}=f \text { in }(0, T) \times \Omega_{2} \text {, ergänzt durch } \\
\text { Anfangs- und Randbedingung auf }(0, T) \times \partial \Omega_{2} \cap \partial \Omega \text { für } u_{2}^{k} \text { sowie } \\
\text { Austauschrandbedingung } \Phi_{2}\left(u_{2}^{k}\right)=\Psi_{2}\left(u_{1}^{k}, u_{2}^{k-1}\right) \text { auf }(0, T) \times \partial \Omega_{1} \cap \partial \Omega_{2} .
\end{array}\right.
$$

Dabei repräsentieren die Randfunktionen $\Phi_{i}$ und $\Psi_{i}, i=1,2$, zu bestimmende Austauschrandbedingungen. Eine der wesentlichen Möglichkeiten der Gebietszerlegung, nämlich die Teilgebiete der Zerlegung numerisch unterschiedlich zu behandeln, wird auf diese Weise auch für die Zeitdiskretisierung verwirklicht. Insbesondere im Zusammenhang mit expliziter Zeitdiskretisierung behält man so die Freiheit, die Zeitschrittweiten von Teilgebiet zu Teilgebiet unterschiedlich zu wählen. Durch geeignete Wahl der Austauschrandbedingungen wird gewährleistet, daß das Verfahren eine Folge $\left(\left(u_{1}^{k}, u_{2}^{k}\right)\right)_{k \in \mathbb{N}}$ generiert, die komponentenweise über dem entsprechenden Teilgebiet gegen die Lösung des globalen Problems konvergiert. Die Randfunktion $\Psi_{i}$ kann von den Lösungen beider Teilgebiete abhängen.

Das oben schematisch angegebene Verfahren ist multiplikativ. Die Teilprobleme werden nacheinander gelöst, wobei zur Bildung der Randwerte für das zweite Teilproblem die Lösung des ersten Teilproblems aus dem aktuellen Iterationsschritt herangezogen wird. Daneben kann es additiv formuliert werden. Dann werden sämtliche Teilprobleme simultan gelöst ausschließlich mit Randwerten, die mit Hilfe von Lösungen aus dem vorangegangenen Iterationsschritt gebildet werden.

Die Anwendung der Waveform-Relaxation-Methode im Zusammenhang mit Gebietszerlegungsalgorithmen für Evolutionsprobleme ist in den letzten Jahren ausgiebig zum Gegenstand der Forschung geworden. In der klassischen Variante werden überlappende Teilgebiete genutzt und an den künstlichen Rändern Dirichlet-Werte ausgetauscht (in der Literatur 
Schwarz waveform relaxation method, siehe [40, 25, 30, 31]). Für zeitabhängige KonvektionsDiffusions-Probleme ergeben sich dabei superlineare Konvergenzraten ([31]), was Resultaten für die Anwendung von Waveform-Relaxation-Algorithmen auf Systeme gewöhnlicher Differentialgleichungen entspricht ([55], [10]). Es zeigt sich jedoch, daß der Austausch von ausschließlich Dirichlet-Werten in bestimmten Fällen den Informationsaustausch zwischen den Teilgebieten hemmt und folglich die Konvergenzgeschwindigkeit verringert. Speziell für die Wellengleichung und für Konvektions-Diffusions-Reaktions-Gleichungen wird in der Arbeit [26] demonstriert, wie im ersten Fall an künstlichen Rändern Wellen fälschlich reflektiert und im zweiten Fall die Konvektion und Diffusion von Information durch die künstlichen Ränder gehemmt wird. Die Konvergenzgeschwindigkeit kann deutlich gesteigert werden, indem man die sogenannte optimierte Schwarz-Methode ([54, 39]), bei der absorbierende Randbedingungen an den künstlichen Rändern genutzt werden, auf Evolutionsprobleme erweitert. Man spricht dann von optimierten Schwarz-Waveform-Relaxations-Methoden. Als besonders effektiv hat sich die Approximation der absorbierenden Randbedingungen auf Grundlage einer Konvergenzratenoptimierung erwiesen, eingeführt ebenfalls in [26]. Weitere Anwendung findet man etwa in [27] für die Wellengleichung und in [29, 48, 49, 28] für KonvektionsDiffusions-Reaktions-Gleichungen.

Im Rahmen dieser Arbeit führen wir in Anlehnung an Verfahren für stationäre Probleme (siehe [2]) ein nicht überlappendes Dirichlet-Robin-Iterationsverfahren vom WaveformRelaxation-Typ für zeitabhängige Probleme ein. Die Untersuchung eines Dirichlet-RobinVerfahrens in diesem Kontext ist uns aus der Literatur nicht bekannt. Auch scheint uns in diesem Zusammenhang die Nutzung von Finite-Volumen-Verfahren neu. Wir führen die Betrachtung für zwei Teilgebiete, auf einem Teilgebiet werden somit Dirichlet-Randwerte und auf dem anderen Robin-Randbedingungen vorgegeben. Das konstruierte Verfahren ist ein relaxierter Algorithmus, bei dem $u_{1}^{k}=\Phi_{1}\left(u_{1}^{k}\right)=\Psi_{1}\left(u_{1}^{k-1}, u_{2}^{k-1}\right)=\sigma u_{2}^{k-1}+(1-\sigma) u_{1}^{k-1}$ mit $\sigma \in(0,1]$ auf dem Austauschrand gilt. Für das zweite Teilgebiet beschreibt $\Phi_{2}$ in unserem Fall eine Robin-Bedingung. Teil dieser Bedingung ist ein Term mit einer frei wählbaren Randfunktion $\gamma$, die generell die Konvergenz des Verfahrens sicherstellen soll. Entsprechende Untersuchungen führen wir in dieser Arbeit. Darüber hinaus beeinflußt $\gamma$ entscheidend die Konvergenzrate. Untersuchungen dazu werden ebenfalls Inhalt dieser Arbeit sein. Bei den in der Literatur präsenten Fällen wird die optimierte Schwarz-Methode stets für sämtliche Teilgebiete herangezogen. In der vorliegenden Arbeit nutzen wir absorbierende Randbedingungen jedoch nur für ein Teilgebiet, nämlich um die Austauschrandfunktion $\gamma$ der Robin-Bedingung optimal bezüglich der Konvergenzrate zu bestimmen. Optimierte Schwarz-Methoden bieten sich dabei an, da die einfachste Approximation absorbierender Randbedingungen, bei der nur konstante Glieder berücksichtigt werden, gerade auf Robin-Bedingungen führen. Dieses Vorgehen im Zusammenhang mit einem Dirichlet-Robin-Algorithmus und einem relaxierten Verfahren erscheint uns neu, insbesondere die Berücksichtigung des Relaxationsparameters und der Größenverhählnisse der Teilgebiete bei der Optimierung. Wie in den Arbeiten [48, 49] übertragen wir die Techniken aus [26] auf zweidimensionale Probleme.

\section{Aufbau der Arbeit}

Die vorliegende Arbeit gliedert sich in zwei Teile, einem zu Finite-Volumen-Verfahren und einem zu Gebietszerlegungstechniken unter Einbeziehung von Finite-Volumen-Verfahren. Die Gliederung der beiden Teile in Kapitel ist wie folgt. Kapitel 1 dient der Darstellung der untersuchten Problemklasse von zeitabhängigen Konvektions-Diffusions-Reaktions-Gleichungen, 
Einleitung

des verallgemeinerten mathematischen Kontextes, in dem diese behandelt werden, sowie der entsprechenden semidiskreten Probleme bei zunächst nur räumlicher Diskretisierung.

In Kapitel $\mathbf{2}$ geben wir ein Finite-Volumen-Verfahren für zeitabhängige KonvektionsDiffusions-Reaktions-Probleme an, indem wir Finite-Volumen-Räume auf Grundlage einer Finite-Element-Ausgangstriangulierung konstruieren. Es werden Regularitäts- und Gleichgewichtsbedingungen an die Kontrollvolumina sowie abschließend die Konstruktion eines speziellen Boxgitter-Typs, des Donald-Diagramms, angegeben.

Wie bereits erwähnt, läßt sich die Finite-Volumen-Diskretisierung als ein konformer verallgemeinerter Galerkin-Ansatz formulieren. Wir nutzen in Kapitel 3 die bekannten Techniken von Anwendungen auf elliptische Probleme zur Abschätzung der Konsistenzfehler, die aus den unterschiedlichen Linerar- und Bilinearformen resultieren. Wir leiten a-prioriFehlerabschätzungen sowohl für die nur räumlich diskretisierten Gleichungen (semidiskretes Problem) als auch für die mit Hilfe des $\theta$-Schemas volldiskretisierten Gleichungen her. Dabei können die aus der Literatur bekannten Ordnungen für Abschätzungen im Fall von FiniteElement-Diskretisierung mit stückweise affinen Ansatzfunktionen reproduziert werden.

Kapitel 4 schließt mit einer Upwind-Modifikation des Verfahrens zur Stabilisierung in konvektionsdominanten Fällen an. Die freie Wahl der Upwind-Funktion, die in diese Modifikation eingeht, sorgt dabei für Kontrolle über das Maß des „Upwindings“. Wir zeigen, daß die Stabilität des Verfahrens unabhängig von der Stärke der Diffusion gewährleistet ist, und leiten analog zu Kapitel 3 a-priori-Fehlerabschätzungen ab. Es ergibt sich eine Fehlerordnung von $O(h)$ bezüglich der $L^{2}$-Norm.

In Kapitel 5 entwickeln wir ein Gebietszerlegungsverfahren vom Dirichlet-Robin-Typ bezüglich nichtüberlappender Gebiete für zeitabhängige Probleme. Aus einer Formulierung von Teilproblemen für zwei Teilgebiete, die durch Randbedingungen gerade so miteinander gekoppelt sind, daß sich Äquivalenz zum ursprünglichen Problem ergibt (Zweigebietsformulierung) leiten wir den eigentlichen iterativen Gebietszerlegungsalgorithmus ab.

Kapitel 6 ist dann der Konvergenzanalyse des Verfahrens gewidmet. Wir geben Fehlergleichungen auf dem Austauschrandrand in Bezug auf die Lösung des Zweigebietsformulierung des Ausgangsproblems an und konstruieren geeignete Steklov-Poincaré-Operatoren speziell für den hier betrachteten zeitabhängingen Fall. Mit Hilfe einer bekannten abstrakten Konvergenzaussage können wir so lineare Konvergenz ableiten, allerdings nicht ohne eine Annahme bezüglich der Steklov-Poincaré-Operatoren, die sinnvoll erscheint, jedoch offen bleibt. Eine Grundlage für eine bezüglich der Konvergenzrate optimierten Wahl der freien Austauschrandfunktion $\gamma$ wird mit diesen Erbebnissen jedoch nicht gelegt.

Den Inhalt von Kapitel 7 bildet die Konvergenzanalyse auf diskretem Level. Zur Anwendung kommt dabei die Finite-Volumen-Technik, wie sie im ersten Teil der Arbeit entwickelt wurde. Bei der Übertragung der Konvergenzuntersuchung des vorausgegangenen Kapitels auf den semidiskretisierten Fall kommen erneut die Konsistenzfehlerabschätzungen des Finite-VolumenVerfahrens zum Tragen. Numerische Ergebnisse schließen das Kapitel ab und stützen das unter einer Annahme erzielte theoretische Konvergenzresultat. Dabei wurden sämtliche numerischen Beispiele (und auch die des nächsten Kapitels) jedoch mit Hilfe einer verifizierten Finite-Element-Diskretisierung auf Grundlage der Programmpakete MATLAB/FEMLAB statt einer Finite-Volumen-Diskretisierung berechnet. 
In Kapitel 8 untersuchen wir eine Strategie zur optimierten Wahl von $\gamma$, zunächst für eindimensionale, anschließend für zweidimensionale Probleme. Betrachtet werden dazu Konvektions-Diffusions-Reaktions-Probleme mit konstanten Koeffizienten. Es lassen sich für jeden Iterationsschritt exakte Lösungen der Teilprobleme angeben und daraus ein optimaler Randoperator ableiten, der jedoch einen nicht lokalen Operator darstellt. Durch Approximation dieses Operators gelangen wir mittels eines Min-max-Problems zu optimierten RobinBedingungen, in Abhängigkeit vom Relaxationsparameter des Gebietszerlegungsalgorithmus. Abschließend vergleichen wir auf diese Weise bestimmte Austauschrandparameter $\gamma$ mit Ergebnissen aus numerischen Tests. 


\section{Teil I}

Finite-Volumen-Verfahren 



\section{Kapitel 1}

\section{Zeitabhängige Konvektions-Diffusions-Probleme}

Im ersten Kapitel stellen wir zeitabhängige Konvektions-Diffusions-Reaktions-Probleme und den mathematischen Kontext, in dem diese im verallgemeinerten Sinne behandelt werden können, vor. Wir gehen auf charakteristische Eigenschaften der zugrunde liegenden Gleichungen ein und zeichnen unser Vorgehen bei deren Diskretisierung vor.

\subsection{Kontinuierliches Problem}

Bereits für elliptische Probleme ist der klassische Lösungsbegriff (Lösung punktweise formulierter Probleme) sehr restriktiv bezüglich der Voraussetzungen an die Problemdaten. Beispielsweise versagt dieser für das Poisson-Problem mit unstetigem Quellterm oder unstetiger Dirichletscher Randbedingung. Abhilfe schafft ein verallgemeinerter Lösungsbegriff mit angepassten Funktionenräumen.

Auf parabolische Probleme in punktweiser Formulierung übertragen sich die genannten Einschränkungen. Hinzu treten Unzulänglichkeiten im Falle von unstetigen Anfangsbedingungen oder unstetigem Übergang zwischen Anfangs- und Randbedingung. Es ist also auch bezüglich der Zeit eine Betrachtung im verallgemeinerten Sinne nötig.

Den verallgemeinerten Rahmen zur Behandlung zeitabhängiger Probleme stellen wir hier kurz für Evolutionsgleichungen 1. Ordnung dar. Einzelheiten zu den betrachteten Funktionenräumen finden sich im Anhang (siehe A.2 und A.3).

Es sei $\Omega \subseteq \mathbb{R}^{d}$ ein beschränktes Lipschitz-Gebiet und $T \in(0, \infty)$. Unter den Voraussetzungen:

(E1) $V$ ist ein Hilbertraum, stetig und dicht eingebettet in $L^{2}(\Omega)$,

(E2) $a$ ist eine stetige und koerzive Bilinearform auf $V \times V$, es gibt also Konstanten $M, \alpha>0$ mit

$$
|a(u, v)| \leqslant M\|u\|_{V}\|v\|_{V}, \quad a(v, v) \geqslant \alpha\|v\|_{V}^{2} \quad \text { für alle } u, v \in V,
$$


(E3) $F \in L^{2}\left(0, T ; V^{*}\right)$

betrachten wir Evolutionsgleichungen 1. Ordnung der Form:

Finde $u \in H^{1}(0, T ; V)$ mit

$$
\left\{\begin{aligned}
\frac{d}{d t}(u(t), v)+a(u(t), v) & =\langle F(t), v\rangle \quad \text { für fast alle } t \in(0, T) \text { und alle } v \in V \\
u(0) & =u_{0} \in L^{2}(\Omega)
\end{aligned}\right.
$$

Die Anfangbedingung $u(0)=u_{0}$ ist sinnvoll wegen der stetigen Einbettung von $H^{1}(0, T ; V)$ in $C\left([0, T] ; L^{2}(\Omega)\right.$ ) (siehe A.3.3). Der folgende Satz gibt Antwort auf die Frage nach der Lösbarkeit von (1.1) (vergl. [67], Theorem 23 A).

Satz 1.1.1 Sind die Voraussetzungen (E1) - (E3) erfüllt, so besitzt das Evolutionsproblem (1.1) genau eine Lösung $u \in H^{1}(0, T ; V)$. Diese hängt stetig von den Daten ab, $d$. $h$.

$$
\|u\|_{H^{1}(0, T ; V)} \leqslant C\left(\left\|u_{0}\right\|_{0}+\|F\|_{L^{2}\left(0, T ; V^{*}\right)}\right)
$$

mit einer Konstante $C>0$.

Bemerkung 1.1.2 Verschärft man die Voraussetzung (E3) zu

$(\mathrm{E} 3)^{\infty} F \in L^{2}\left(0, t ; V^{*}\right)$ für alle $t \geqslant 0$,

und ist $u$ dann Lösung von (1.1), so läßt sich für jedes $\lambda \in\left[0, \frac{\alpha}{C_{C I}^{2}}\right)$ die Abschätzung

$$
\|u(t)\|_{0}^{2}+\int_{0}^{t} e^{2 \lambda(s-t)}\|u(s)\|_{V}^{2} d s \leqslant C\left(e^{-2 \lambda t}\left\|u_{0}\right\|_{0}^{2}+\int_{0}^{t} e^{2 \lambda(s-t)}\|F(s)\|_{V^{*}}^{2} d s\right), \quad t \geqslant 0
$$

herleiten, wobei $C_{C I}$ die Konstante der in (E1) geforderten stetigen Einbettung bezeichnet. Dies zeigt, daß der Einfluß der Anfangsbedingung exponientiell abklingt, die Glättungseigenschaft parabolischer Probleme. Sie wird bei den a-priori-Abschätzungen im Abschnitt 3.3 wieder hervortreten.

Insbesondere gilt für je zwei Lösungen $u, \tilde{u}$ zu identischem Quellterm $F$ und Anfangsbedingung $u_{0}$ bzw. $\tilde{u}_{0}$

$$
\|u(t)-\tilde{u}(t)\|_{0}^{2} \leqslant C e^{-2 \lambda t}\left\|u_{0}-\tilde{u}_{0}\right\|_{0}^{2} .
$$

Dies macht man sich zunutze, wenn man die Lösung $u_{\infty}$ eines elliptischen Problems

$$
a\left(u_{\infty}, v\right)=\langle F, v\rangle, \quad \text { für alle } v \in V,
$$

$F \in V^{*}$, durch Langzeitintegration des entsprechenden parabolischen Problems

$$
\frac{d}{d t}(u(t), v)+a(u(t), v)=\langle F, v\rangle, \quad \text { für alle } v \in V
$$

zu beliebiger Anfangsbedingung $u_{0}$ berechnet. Denn mit obiger Argumentation gilt dann

$$
\left\|u(t)-u_{\infty}\right\|_{0}^{2} \leqslant C e^{-2 \lambda t}\left\|u_{0}-u_{\infty}\right\|_{0}^{2} .
$$


Als wesentliches Beispiel betrachten wir Konvektions-Diffusions-Reaktions-Probleme über dem Raum $V=H_{0}^{1}(\Omega)$ :

Finde $u \in H^{1}(0, T ; V)$, so daß

$$
\left\{\begin{aligned}
\frac{d}{d t}(u(t), v)+a(u(t), v) & =(f(t), v) \quad \text { für fast alle } t \in(0, T) \text { und alle } v \in V \\
u(0) & =u_{0} \in L^{2}(\Omega)
\end{aligned}\right.
$$

mit

$$
a(u, v):=\int_{\Omega} \nabla v \cdot(A \nabla u-u b)+c u v d x
$$

und den Anforderungen an die Daten

$$
a_{i j}, b_{j}, \nabla \cdot b, c \in L^{\infty}(\Omega) \text { und } f \in L^{2}\left(Q_{T}\right) \text { sowie } a_{i j}=a_{j i} \text { für jedes } i, j \in \underline{d} \text {. }
$$

Dabei bezeichnet $Q_{T}$ den Raum-Zeit-Zylinder $(0, T) \times \Omega$.

Für eine Lösung $u \in H^{1}(0, T ; V)$ von (1.2) gilt

$$
\int_{\Omega} \nabla v \cdot(A \nabla u-u b) d x=\int_{\Omega}\left(f-u^{\prime}-c u\right) v d x \text { für jedes } v \in C_{0}^{\infty}(\Omega)
$$

fast überall in $(0, T)$. Also ist $f-u^{\prime}-c u$ die schwache Divergenz von $-A \nabla u+u b$ fast überall in $(0, T)$, d. h.

$$
L u:=-\nabla \cdot(A \nabla u-u b)+c u=f-u^{\prime} \quad \text { in } L^{2}(0, T ; V) .
$$

Somit ist (1.2) - (1.4) eine verallgemeinerte Aufgabenstellung zum Anfangsrandwertproblem

$$
\begin{cases}u^{\prime}(t)+L u(t)=f(t) & \text { in } Q_{T} \\ u(t)=0 & \text { auf } \partial \Omega \\ u(0)=u_{0} & \text { in } \Omega\end{cases}
$$

mit Diffusionsmatrix $A$, Konvektionsvektor $b$ und Reaktionskoeffizient $c$.

Ist $\Omega^{\prime}$ ein Teilgebiet von $\Omega$, so sei

$$
a_{\Omega^{\prime}}(u, v):=\int_{\Omega^{\prime}} \nabla v \cdot A \nabla u+\left(\frac{1}{2} \nabla \cdot b+c\right) u v d x+\frac{1}{2} \int_{\Omega^{\prime}} v b \cdot \nabla u-u b \cdot \nabla v d x
$$

für alle $u, v \in H^{1}\left(\Omega^{\prime}\right)$. Wegen (1.4) ergibt sich mit Produktregel und partieller Integration

$$
\int_{\Omega} v \nabla \cdot(u b) d x=\frac{1}{2} \int_{\Omega} u v \nabla \cdot b+v b \cdot \nabla u-u b \cdot \nabla v d x
$$

für $u, v \in H_{0}^{1}(\Omega)$ und mithin die Darstellung

$$
a(u, v)=a_{\Omega}(u, v) .
$$


Die Koerzivität der Bilinearform $a_{\Omega^{\prime}}$, insbesondere also auch die von $a$, können wir gewährleisten durch die zusätzlichen Bedingungen

$$
\text { (i) } \eta:=\underset{\Omega}{\operatorname{essinf}} q>0 \text { mit } q:=\frac{1}{2} \nabla \cdot b+c, \quad(i i) \varepsilon:=\underset{\Omega}{\operatorname{essinf}}\left\{\frac{y \cdot A y}{|y|^{2}} \mid y \in \mathbb{R}^{d} \backslash\{0\}\right\}>0 .
$$

Wir erhalten das

Lemma 1.1.3 Es sei $\Omega^{\prime} \subseteq \Omega$ ein Gebiet. Unter den Voraussetzungen (1.4) und (1.8) genügt dann die Bilinearform $a_{\Omega^{\prime}}$ den Relationen

$$
\left|a_{\Omega^{\prime}}(u, v)\right| \leqslant M_{\Omega^{\prime}}\|u\|_{1, \Omega^{\prime}}\|v\|_{1, \Omega^{\prime}}, \quad a_{\Omega^{\prime}}(v, v) \geqslant \alpha_{\Omega^{\prime}}\|v\|_{1, \Omega^{\prime}}^{2}
$$

für alle $u, v \in H^{1}\left(\Omega^{\prime}\right)$ mit

$$
\begin{aligned}
M_{\Omega^{\prime}} & =\max _{i, j \in \underline{d}}\left\|a_{i j}\right\|_{\infty, \Omega^{\prime}}+\|q\|_{\infty, \Omega^{\prime}}+\|b\|_{\infty, \Omega^{\prime}}, \\
\alpha_{\Omega^{\prime}} & =\min \{\varepsilon, \eta\} .
\end{aligned}
$$

Bemerkung 1.1.4 Im Fall eines elliptischen Operators $L$ bedeutet die Koerzivitätsforderung aus (E2) an die Bilinearform a keine entscheidende Einschränkung. Die Voraussetzungen (1.4) und (1.8) (ii) sichern eine Gårding-Ungleichung (schwache Koerzivität)

$$
a(v, v)+\lambda\|v\|_{0}^{2} \geqslant \alpha\|v\|_{1}^{2}, \quad \lambda \geqslant 0, \alpha>0 .
$$

Denn es ist dann wegen $a(u, v)=a_{\Omega}(u, v)$ und mit der Poincaré-Friedrich-Ungleichung (Satz A.2.6)

$$
\begin{aligned}
a(v, v) & \geqslant \varepsilon|v|_{1}^{2}+\int_{\Omega}\left(\frac{1}{2} \nabla \cdot b+c\right) v^{2} d x \\
& \geqslant \frac{\varepsilon}{1+C_{P F}}\|v\|_{1}^{2}-\|q\|_{\infty}\|v\|_{0}^{2} .
\end{aligned}
$$

Offensichtlich löst $u$ genau dann das Problem (1.2), wenn $u_{\lambda}$ Lösung ist von

$$
\left\{\begin{aligned}
\frac{d}{d t}\left(u_{\lambda}(t), v\right)+a_{\lambda}(u(t), v) & =\left(e^{-\lambda t} f(t), v\right) \quad \text { für fast alle } t \in(0, T) \text { und alle } v \in V \\
u_{\lambda}(0) & =u_{0} \in L^{2}(\Omega)
\end{aligned}\right.
$$

mit $u_{\lambda}(t):=e^{-\lambda t} u(t)$ fast überall in $(0, T)$ und $a_{\lambda}(u, v):=a(u, v)+\lambda(u, v)$. Wegen

$a$ schwach koerziv $\Longleftrightarrow a_{\lambda}$ koerziv

kann man sich daher stets auf ein Problem zurückziehen, welches der Bedingung (E2) genügt.

Im weiteren Verlauf der Arbeit werden wir häufig eine problemangepaßte Norm nutzen, die den Einfluß der Stärke von Diffusion und Konvektion erkennen läßt. Dazu gelte die Voraussetzung (1.8). Zudem ist die Diffusionsmatrix $A$ nach (1.4) symmetrisch. Mit der Bezeichnung 


$$
\left\|A^{\frac{1}{2}} \nabla v\right\|_{0, \Omega}^{2}:=\int_{\Omega} \nabla v \cdot A \nabla v d x
$$

gilt daher die Cauchy-Schwarzsche Ungleichung

$$
\left|\int_{\Omega} \nabla v \cdot A \nabla u d x\right| \leqslant\left\|A^{\frac{1}{2}} \nabla u\right\|_{0, \Omega}\left\|A^{\frac{1}{2}} \nabla v\right\|_{0, \Omega},
$$

und durch

$$
\|v\|_{\varepsilon, \Omega}^{2}:=\left\|A^{\frac{1}{2}} \nabla v\right\|_{0, \Omega}^{2}+\|\sqrt{q} v\|_{0, \Omega}^{2}
$$

wird eine Norm auf $H^{1}(\Omega)$ definiert. Wird aus dem Zusammenhang klar, welches Gebiet der Norm zugrunde liegt, schreiben wir abkürzend $\|v\|_{\varepsilon}$ statt $\|v\|_{\varepsilon, \Omega}$.

Wir passen zwei übliche a-priori-Abschätzungen für Konvektions-Diffusions-ReaktionsProbleme an diese Norm $\|\cdot\|_{\varepsilon}$ an. Dies dient insbesondere als Vorbereitung zu den Fehlerabschätzungen im späteren Abschnitt 3.3.

Lemma 1.1.5 Es gelte (1.8). Dann genügt die Lösung u des Problems (1.2)-(1.4) für $t \in$ $(0, T)$ den a-priori-Abschätzungen

$$
\|u(t)\|_{0}^{2}+\int_{0}^{t}\|u(s)\|_{\varepsilon}^{2} d s \leqslant\left\|u_{0}\right\|_{0}^{2}+\frac{1}{\eta} \int_{0}^{t}\|f(s)\|_{0}^{2} d s
$$

und

$$
\begin{aligned}
\left\|A^{\frac{1}{2}} \nabla u(t)\right\|_{0}^{2}+\int_{0}^{t}\left\|u^{\prime}(s)\right\|_{0}^{2} d s \leqslant & \left\|A^{\frac{1}{2}} \nabla u_{0}\right\|_{0}^{2}+2 \int_{0}^{t}\|f(s)\|_{0}^{2} d s \\
& +4 \max \left\{\frac{1}{\varepsilon}, \frac{1}{\eta}\right\}\left(\|\nabla \cdot b\|_{\infty}^{2}+\|b\|_{\infty}^{2}+\|c\|_{\infty}^{2}\right) \int_{0}^{t}\|u(s)\|_{\varepsilon}^{2} d s .
\end{aligned}
$$

Beweis. $\quad$ Mit partieller Integration bezüglich der Zeit (Satz A.3.4) erhält man

$$
\frac{1}{2} \frac{d}{d t}\|u(t)\|_{0}^{2}=\frac{1}{2} \frac{d}{d t}(u(t), u(t))=\left\langle u^{\prime}(t), u(t)\right\rangle .
$$

Daher folgt aus (1.2) mit der Wahl $v=u$ sowie wegen $a(u, u)=a_{\Omega}(u, u)$ und (1.8)

$$
\begin{aligned}
\frac{1}{2} \frac{d}{d t}\|u(t)\|_{0}^{2}+\|u(t)\|_{\varepsilon}^{2} & =(f(t), u(t)) \leqslant \frac{1}{\sqrt{\eta}}\|f(t)\|_{0}\|u(t)\|_{\varepsilon} \\
& \leqslant \frac{1}{2}\left(\frac{1}{\eta}\|f(t)\|_{0}^{2}+\|u(t)\|_{\varepsilon}^{2}\right) .
\end{aligned}
$$

Integration über $(0, t)$ liefert

$$
\|u(t)\|_{0}^{2}-\left\|u_{0}\right\|_{0}^{2}+\int_{0}^{t}\|u(s)\|_{\varepsilon}^{2} d s \leqslant \frac{1}{\eta} \int_{0}^{t}\|f(s)\|_{0}^{2} d s,
$$


und dies ist die erste Behauptung.

Für die zweite Abschätzung ziehen wir Proposition 11.1.1 in [57] und den zugehörigen Beweis heran. Mit partieller Integration bezüglich der Zeit ergibt sich (siehe Gl. (11.1.16) im genannten Beweis)

$$
\begin{aligned}
& \left\|u^{\prime}(t)\right\|_{0}^{2}+\frac{1}{2} \frac{d}{d t}\left\|A^{\frac{1}{2}} \nabla u(t)\right\|_{0}^{2} \\
& \quad \leqslant\|f(t)\|_{0}\left\|u^{\prime}(t)\right\|_{0}+\|\nabla \cdot(u(t) b)\|_{0}\left\|u^{\prime}(t)\right\|_{0}+\|c\|_{\infty}\|u(t)\|_{0}\left\|u^{\prime}(t)\right\|_{0}
\end{aligned}
$$

Wegen $\nabla \cdot b \in L^{\infty}(\Omega)$ gilt mit der Produktregel sowie $\beta_{\infty}:=\left(\|\nabla \cdot b\|_{\infty}^{2}+\|b\|_{\infty}^{2}\right)^{\frac{1}{2}}$ und $C_{\varepsilon, \eta}:=\max \left\{\frac{1}{\varepsilon}, \frac{1}{\eta}\right\}$

$$
\begin{aligned}
\|\nabla \cdot(u b)\|_{0} & \leqslant\|\nabla \cdot b\|_{\infty}\|u\|_{0}+\|b\|_{\infty}|u|_{1} \\
& \leqslant\left(\|\nabla \cdot b\|_{\infty}^{2}+\|b\|_{\infty}^{2}\right)^{\frac{1}{2}}\left(\frac{1}{\eta}\|\sqrt{q} u\|_{0}^{2}+\frac{1}{\varepsilon}\left\|A^{\frac{1}{2}} \nabla u\right\|_{0}^{2}\right)^{\frac{1}{2}} \\
& \leqslant \beta_{\infty} \sqrt{C_{\varepsilon, \eta}}\|u\|_{\varepsilon}
\end{aligned}
$$

fast überall in $(0, T)$. Somit folgt unter Anwendung der Youngschen Ungleichung

$$
\begin{aligned}
\left\|u^{\prime}(t)\right\|_{0}^{2}+\frac{1}{2} \frac{d}{d t}\left\|A^{\frac{1}{2}} \nabla u(t)\right\|_{0}^{2} \leqslant & \|f(t)\|_{0}\left\|u^{\prime}(t)\right\|_{0} \\
& +\left(\beta_{\infty} \sqrt{C_{\varepsilon, \eta}}\|u(t)\|_{\varepsilon}+\|c\|_{\infty}\|u(t)\|_{0}\right)\left\|u^{\prime}(t)\right\|_{0} \\
\leqslant & \|f(t)\|_{0}\left\|u^{\prime}(t)\right\|_{0}+\sqrt{C_{\varepsilon, \eta}}\left(\beta_{\infty}+\|c\|_{\infty}\right)\|u(t)\|\left\|_{\varepsilon}\right\| u^{\prime}(t) \|_{0} \\
\leqslant & \|f(t)\|_{0}^{2}+\frac{1}{4}\left\|u^{\prime}(t)\right\|_{0} \\
& +C_{\varepsilon, \eta}\left(\beta_{\infty}+\|c\|_{\infty}\right)^{2}\|u(t)\|_{\varepsilon}^{2}+\frac{1}{4}\left\|u^{\prime}(t)\right\|_{0}^{2} .
\end{aligned}
$$

Nach Integration über $(0, t)$ erhält man

$$
\begin{aligned}
\int_{0}^{t}\left\|u^{\prime}(s)\right\|_{0}^{2} d s & +\left\|A^{\frac{1}{2}} \nabla u(t)\right\|_{0}^{2}-\left\|A^{\frac{1}{2}} \nabla u(0)\right\|_{0}^{2} \\
& \leqslant 2\left(\int_{0}^{t}\|f(s)\|_{0}^{2} d s+2 C_{\varepsilon, \eta}\left(\|\nabla \cdot b\|_{\infty}^{2}+\|b\|_{\infty}^{2}+\|c\|_{\infty}^{2}\right) \int_{0}^{t}\|u(s)\|_{\varepsilon}^{2} d s\right)
\end{aligned}
$$

also die Abschätzung (1.13).

Besondere Aufmerksamkeit verlangen solche Fälle von (1.2) - (1.4), bei denen der Diffusionsterm klein gegenüber dem Konvektionsterm wird, bei denen also $\varepsilon \ll\|b\|_{\infty}$ gilt. Es treten schmale Regionen in $\Omega$ auf, in denen sich die Lösung oder deren Gradient abrupt ändert. Im Grenzwert $\varepsilon \rightarrow 0$ entsteht ein hyperbolisches Problem 1. Ordnung, bei dem die ursprüngliche Randbedingung nur noch auf einer Teilmenge $(0, T) \times \Gamma, \Gamma \subset \partial \Omega$, dem Einströmrand, erfüllt werden kann. 
In solchen Fällen, bei denen abhängig vom einem Parameter völlig unterschiedliche Charaktere der Problemgleichungen zu Tage treten, spricht man bekanntermaßen von singulär gestörten Problemen. Durch Standard Galerkin-Verfahren wird hier im Gegensatz zu diffusionsdominierten Problemen die Lösung nicht angemessen approximiert (vergl. [59], II.4 und III.3).

Abschließend geben wir ein Beispiel, das sowohl die Glättungseigenschaft als auch das Grenzschichtverhalten illustriert.

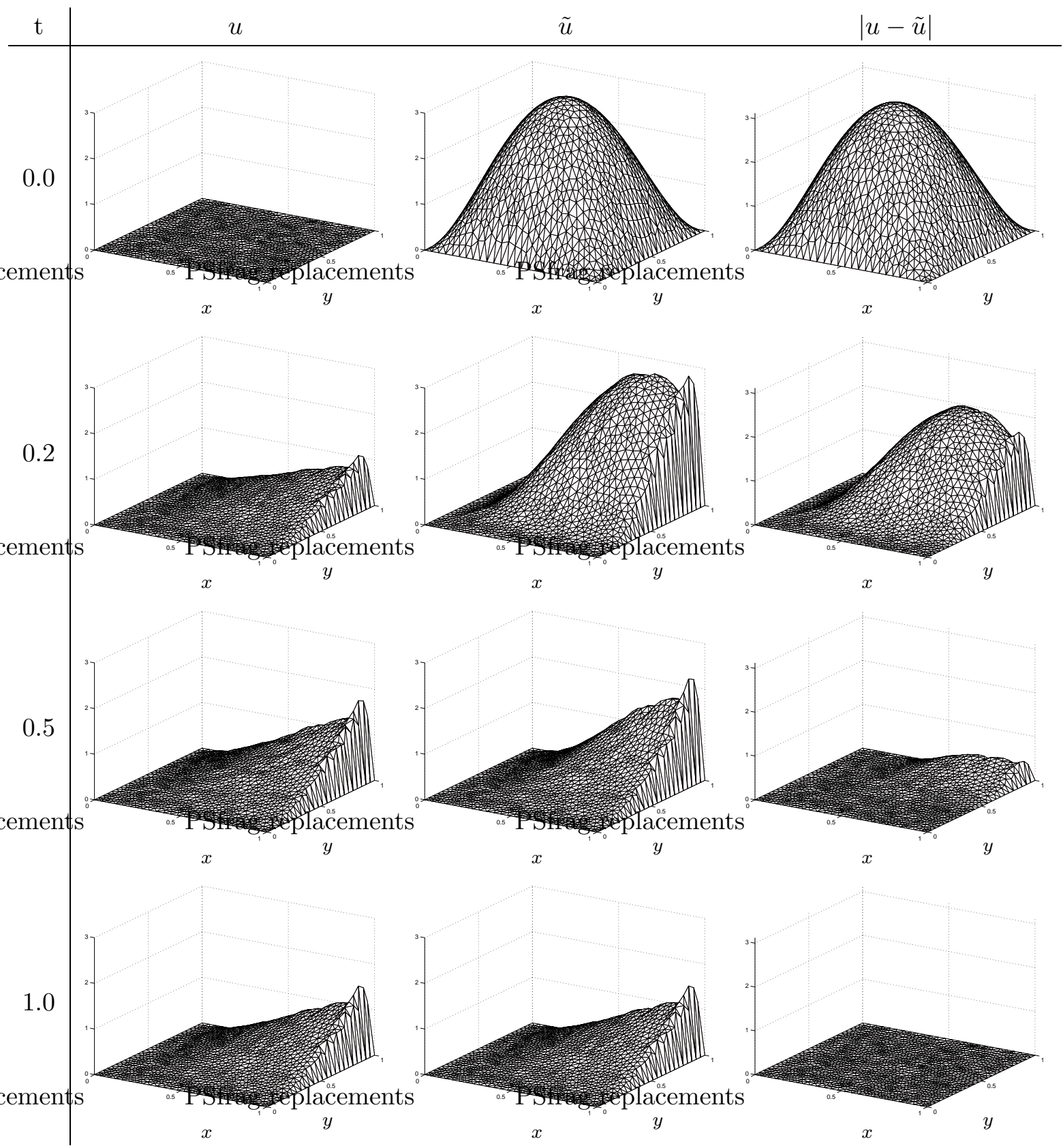

Abbildung 1.1: Numerische Approxmimationen zweier Lösungen von (1.14) zu unterschiedlichen Anfangswerten und deren Abstand zueinander. 
Beispiel 1.1.6 In diesem Beispiel sei $\Omega=(0,1)^{2} \cdot u$ und $\tilde{u}$ seien die Lösungen von

$$
\left\{\begin{array}{l}
u^{\prime}(t)-\varepsilon \Delta u(t)-(1,2)^{T} \cdot \nabla u(t)+u(t)=20 \sin ^{2}(5 \pi t) x y \quad \text { in } Q_{T} \\
u(t)=0 \quad \text { auf } \partial \Omega
\end{array}\right.
$$

mit $\varepsilon=10^{-4} \mathrm{zu}$ den Anfangsbedingungen

$$
u_{0}(x, y)=0 \quad \text { bzw. } \quad \tilde{u}_{0}(x, y)=50\left(x-x^{2}\right)\left(y-y^{2}\right) \quad \text { in } \Omega .
$$

Die Abbildung 1.1 zeigt numerische Lösung zu $u_{0}$ und $\tilde{u}_{0}$ sowie den Abstand dieser Lösungen voneinander. Bereits bei $t=1.0$ ist der Unterschied im Einfluß der Anfangsbedingungen abgeklungen. An den Rändern $x=1$ und $y=1$ zeigt sich deutlich eine Grenzschicht.

\subsection{Semidiskretes Problem}

Eine Möglichkeit, die Lösung des Evolutionsproblems (1.1) zu approximieren, besteht darin, durch zunächst nur räumliche Diskretisierung ein System gewöhnlicher Differentialgleichungen aufzustellen. Dieser Weg, häufig vertikale Linienmethode genannt, wird in dieser Arbeit eingeschlagen.

Genauer, zu einem endlichdimensionalen Unterraum $V_{h} \subseteq V$ und einer geeigneten Bilinearform $a_{h}$ auf $V_{h} \times V_{h}$ wird das sogenannte semidiskrete Problem zu (1.1) betrachtet:

Finde $u_{h} \in H^{1}\left(0, T ; V_{h}\right)=\left\{u \in L^{2}\left(0, T ; V_{h}\right) \mid u^{\prime} \in L^{2}\left(0, T ; V_{h}^{*}\right)\right\}$ mit

$$
\left\{\begin{aligned}
\frac{d}{d t}\left(u_{h}(t), v\right)+a_{h}\left(u_{h}(t), v\right) & =\langle F(t), v\rangle \quad \text { für fast alle } t \in(0, T) \text { und alle } v \in V_{h} \\
u_{h}(0) & =u_{0, h} .
\end{aligned}\right.
$$

Dabei stellt $u_{0, h} \in V_{h}$ eine geeignete Approximation des Anfangswertes $u_{0}$ dar, etwa die $L^{2}$-Orthogonalprojektion von $u_{0}$ auf $V_{h}$

$$
\left(u_{0, h}-u_{0}, v\right)=0 \quad \text { für alle } v \in V_{h}
$$

oder die Ritz-Projektion von $u_{0}$ auf $V_{h}$ (siehe Lemma 3.3.1).

Die Wahl des Unterraums $V_{h}$ und der Bilinearform $a_{h}$ bestimmt grundsätzlich die Art der räumlichen Diskretisierung. Im Fall von $a_{h} \neq a$ spricht man von einem verallgemeinerten Galerkin-Verfahren.

In dieser Arbeit werden wir sowohl Finite-Element-Diskretisierungen als auch FiniteVolumen-Diskretisierungen heranziehen. Der entsprechende Unterraum $V_{h}$ wird im ersten Fall von global stetigen, elementweise polynomialen Funktionen eines bestimmten maximalen Polynomgrades gebildet, speziell elementweise affinen Funktionen.

Im zweiten Fall liegen zunächst bezüglich der Volumina der Diskretisierung stückweise konstante (global unstetige) Testfunktionen zugrunde. Jedoch kann auch dieser Fall auf den 
Raum der stetigen, elementweise affinen Funktionen zurückgeführt werden, siehe nachfolgendes Kapitel. Somit kann auch die in dieser Arbeit verwendete Finite-Volumen-Diskretisierung auf die Form (1.15) gebracht werden mit einem $V_{h} \subseteq V$.

Das semidiskrete Problem (1.15) ist gleichwertig zu einem System gewöhnlicher Differentialgleichungen in der Zeit. Denn ist $\left\{\psi_{1}, \ldots, \psi_{N_{h}}\right\}$ eine Basis von $V_{h}$, so ist $u_{h} \in L^{2}\left(0, T ; V_{h}\right)$ mit

$$
u_{h}(t)=\sum_{j \in \underline{N_{h}}} x_{j}(t) \psi_{j}
$$

Lösung von (1.15) zum Anfangswert $u_{0, h}=\sum_{j \in \underline{N_{h}}} x_{0, j} \psi_{j}$, genau dann wenn $\mathbf{x} \in \mathbb{R}^{(0, T)}$ mit $\mathbf{x}(t)=\left(x_{j}(t)\right)_{j=1}^{N_{h}}$ das System

$$
\left\{\begin{aligned}
M \mathbf{x}^{\prime}(t)+A \mathbf{x}(t) & =\mathbf{F}(t) \\
\mathbf{x}(0) & =\mathbf{x}_{0}
\end{aligned}\right.
$$

löst. Dabei ist

$$
\begin{aligned}
& M:=\left(\left(\psi_{j}, \psi_{i}\right)\right)_{i, j=1}^{N_{h}}, \quad A:=\left(a_{h}\left(\psi_{j}, \psi_{i}\right)\right)_{i, j=1}^{N_{h}}, \\
& \mathbf{F}(t):=\left(\left\langle F(t), \psi_{i}\right\rangle_{V_{h}}\right)_{i=1}^{N_{h}}, \quad \mathbf{x}_{0}:=\left(x_{0, j}\right)_{j=1}^{N_{h}} .
\end{aligned}
$$

Die Existenz einer eindeutig bestimmten Lösung von (1.15) liefert der

Satz 1.2.1 Der Raum $V$ erfülle Bedingung (E1), und es sei $V_{h}$ ein endlich dimensionaler Unterraum von $V$ sowie $F \in L^{2}\left(0, T ; V^{*}\right)$. Weiterhin sei $a_{h}$ eine koerzive Biliniearform auf $V_{h} \times V_{h}$. Dann hat das semidiskrete Problem (1.15) genau eine Lösung $u_{h} \in H^{1}\left(0, T ; V_{h}\right)$.

Beweis. $\quad$ Vergl. (I) im Beweis von Korollar 23.26 in [67]. 


\section{Kapitel 2}

\section{Finite-Volumen-Verfahren}

In diesem Kapitel führen wir ein Finite-Volumen-Verfahren für Konvektions-DiffusionsReaktions-Probleme ein, indem wir Finite-Volumen-Räume auf Grundlage einer (FiniteElement-)Ausgangstriangulierung konstruieren und anschließend unsere Finite-VolumenDiskretisierung für Probleme der Form (1.2) - (1.4) damit bilden.

Der Vorteil dieser Vorgehensweise ist, daß sich besonders enge Zusammenhänge zur FiniteElement-Diskretisierung mit $P_{1}$-Elementen herausstellen.

Im Rest des Kapitels nennen wir Regularitäts- und Gleichgewichtsbedingungen an die Volumina der Diskretisierung zur Vorbereitung der Konsistenzfehlerabschätzung des nächsten Kapitels und schließen mit der Konstruktion von Volumina eines speziellen Typs, des DonaldDiagramms.

\subsection{Ausgangstriangulierung}

Die Finite-Volumen-Diskretisierung kann im Prinzip auf beliebigen endlichen Zerlegungen aufbauen, also etwa auch auf den Volumina einer vorgegebenen Triangulierung. Grundlage für das in dieser Arbeit verwendete Finite-Volumen-Verfahren sind jedoch Kontrollvolumina, die „dual“" zu einer Triangulierung definiert werden.

Die Terminologie zur Ausgangstriangulierung und einige Bedingungen an deren Triangulierungsmengen werden an dieser Stelle besonders ausführlich aufgeführt, weil sie unmittelbar danach, nämlich in den Abschnitten 2.4 und 2.5, benötigt werden.

Es sei $\Omega \subseteq \mathbb{R}^{d}$ ein polyedrisches Lipschitz-Gebiet. Im folgenden betrachten wir ausschließlich simpliziale Triangulierungen von $\Omega$, d. h. jede Triangulierung auf $\Omega$ bestehe aus regulären $d$-Simplizes. Dabei heißt eine Menge $S \subseteq \mathbb{R}^{d} k$-Simplex, falls sie die konvexe Hülle $\operatorname{conv}\left(\left\{x_{S}^{(0)}, \ldots, x_{S}^{(k)}\right\}\right)$ von $k+1$ Punkten $x_{S}^{(0)}, \ldots, x_{S}^{(k)} \in \mathbb{R}^{d}$, genannt Eckpunkte von $S$, ist und $k \in d+1$ gilt. $S$ heißt regulär, falls die Menge $\left\{x_{S}^{(j)}-x_{S}^{(0)} \mid j \in \underline{d}\right\}$ linear unabhängig ist. Ein $d$-Simplex nennen wir kürzer auch Simplex.

Ist $R$ die konvexe Hülle von $l+1$ der Eckpunkte eines $k$-Simplex $S, l \in k$, so bezeichnen wir $R$ als ein $l$-Randsimplex oder einfach als ein Randsimplex von $S$. 1-Randsimplizes heißen 


\section{Kanten.}

Ist $S=\operatorname{conv}\left(\left\{x_{S}^{(0)}, \ldots, x_{S}^{(d)}\right\}\right) \subseteq \mathbb{R}^{d}$ ein reguläres Simplex, so existieren zu jedem Punkt $x \in \mathbb{R}^{d}$ eindeutig bestimmte Zahlen $\lambda_{0}=\lambda_{0}(x), \ldots, \lambda_{d}=\lambda_{d}(x)$ mit

$$
x=\sum_{j \in d+1} \lambda_{j} x_{S}^{(j)}, \quad \sum_{j \in d+1} \lambda_{j}=1,
$$

genannt die baryzentrische Koordinaten von $x$ bezüglich $S$. Mit diesen gilt

$$
x \in S \Longleftrightarrow \lambda_{j} \geqslant 0 \text { für jedes } j \in d+1 \text {. }
$$

Als Schwerpunkt von $S$ bezeichnet man

$$
\sigma(S):=\frac{1}{k+1} \sum_{j \in k+1} x_{S}^{(j)} .
$$

Unter den geometrischen Größen eines Simplex $T$ kennzeichnen wir den Durchmesser von $T$ mit $h_{T}$ und das Supremum der Radien aller $T$ einbeschriebenen Kugeln mit $\rho_{T}$. Jeder Triangulierung $\mathcal{T}$ läßt sich mit $h:=\max _{T \in \mathcal{T}} h_{T}$ ein Parameter zuordnen. Man schreibt daher auch $\mathcal{T}_{h}$ statt $\mathcal{T}$.

Die Eckpunkte (0-Randsimplizes) der Elemente einer Triangulierung $\mathcal{T}_{h}$ werden Knoten von $\mathcal{T}_{h}$ genannt, deren Anzahl sei $N_{h}$. Abhängig von den Randbedingungen eines Randwertproblems betrachtet man gegebenenfalls die Menge aller Knoten von $\mathcal{T}_{h}$, die im Innern von $\Omega$ liegen. Die Mächtigkeit dieser Menge werde mit $N_{h}^{0}$ bezeichnet. Die Numerierung $i \mapsto a_{i}$ der Knoten $\left\{a_{1}, \ldots, a_{N_{h}}\right\}$ sei stets so gewählt, daß $a_{i} \in \Omega^{\circ}$ genau für $i \in \underline{N_{h}^{0}}$ gilt.

Als weitere Bedingung an die Triangulierung setzen wir voraus, daß der Schnitt je zweier Triangulierungsmengen $T, T^{\prime} \in \mathcal{T}_{h}$ entweder leer oder ein gemeinsames Randsimplex von $T$ und $T^{\prime}$ ist. Genügt eine Triangulierung dieser Bedingung, wird sie zulässige Triangulierung auf $\Omega$ genannt. Sogenannte „hängende Knoten“ sind dann ausgeschlossen. Zudem sei die Triangulierung exakt, also $\Omega$ die Vereinigung aller Triangulierungsmengen aus $\mathcal{T}_{h}$.

Auf $\mathcal{T}_{h}$ definieren wir die Finite-Element-Räume

$$
\begin{aligned}
& P^{k}\left(\mathcal{T}_{h}\right):=\left\{u \in C(\bar{\Omega})|u|_{T} \in P_{k} \text { für jedes } T \in \mathcal{T}_{h}\right\} \\
& P_{0}^{k}\left(\mathcal{T}_{h}\right):=\left\{u \in P^{k}\left(\mathcal{T}_{h}\right) \mid u=0 \text { auf } \partial \Omega\right\}
\end{aligned}
$$

wobei $P_{k}$ den Raum der Polynome über $\mathbb{R}$ maximal vom Grad $k \in \mathbb{N}_{0}$ bezeichnet. Wegen $P^{1}\left(\mathcal{T}_{h}\right) \cong \mathbb{R}^{N_{h}}$ und $P_{0}^{1}\left(\mathcal{T}_{h}\right) \cong \mathbb{R}^{N_{h}^{0}}$ ist $N_{h}=\operatorname{dim} P^{1}\left(\mathcal{T}_{h}\right)$ und $N_{h, 0}=\operatorname{dim} P_{0}^{1}\left(\mathcal{T}_{h}\right) .\left\{\varphi_{1}, \ldots, \varphi_{N_{h}}\right\}$ mit $\varphi_{j}\left(a_{i}\right)=\delta_{i j}$ bezeichnet die nodale Basis von $P^{1}\left(\mathcal{T}_{h}\right)$.

Eine Familie $\left(\mathcal{T}_{h}\right)_{h>0}$ von Triangulierungen auf $\Omega$ heißt regulär, falls ein $\rho>0$ gibt, so daß

$$
\max _{T \in \mathcal{T}_{h}} \frac{h_{T}}{\rho_{T}} \leqslant \rho
$$

für alle $h>0$ gilt.

Schließlich sammeln wir einige Voraussetzungen, auf die nachfolgend häufig zurückgegriffen wird, unter dem Kürzel 
(TR) Es sei $\Omega \subseteq \mathbb{R}^{d}$ ein polyedrisches Lipschitz-Gebiet und $\left(\mathcal{T}_{h}\right)_{h>0}$ eine reguläre Familie zulässiger Triangulierungen darauf, die durch affine Transformation von einem Referenzelement erzeugt wird.

\subsection{Finite-Volumen-Räume}

Die vorgelegte Triangulierung dient nun zur Konstruktion einer Zerlegung von $\Omega$ durch ein endliches „duales“ Mengensystem. Dabei orientieren wir uns am Vorgehen in [9]. Dort werden die dual festgelegten Volumina zunächst allgemeiner gehalten als in [7], [35] und [15], für Konsistenzfehlerabschätzungen nachträglich freilich gewisse Regularitäts- und Gleichgewichtsbedingungen gefordert.

Ein Mengensystem $\mathcal{B}_{h}=\left\{B_{1}, \ldots, B_{N_{h}}\right\} \subseteq \wp(\bar{\Omega})$ heißt duales Boxgitter zu $\mathcal{T}_{h}$, falls gilt

(V1) $\mathcal{B}_{h}$ ist eine Zerlegung von $\Omega$ in abgeschlossene Mengen,

(V2) $B_{j}^{\circ}$ ist ein Lipschitz-Gebiet mit $B_{j} \subseteq \overline{B_{j}^{\circ}}$ für jedes $j \in \underline{N_{h}}$,

(V3) $a_{j} \in B_{j}$ für jedes $j \in \underline{N_{h}}$,

(V4) $B_{j} \subseteq \bigcup\left\{T \in \mathcal{T}_{h} \mid a_{j} \in T\right\}$ für jedes $j \in \underline{N_{h}}$.

Ein Element aus $\mathcal{B}_{h}$ heißt dann Kontrollvolumen, finites Volumen oder auch Box. Abbildung 2.1 zeigt schematisch ein duales Boxgitter.

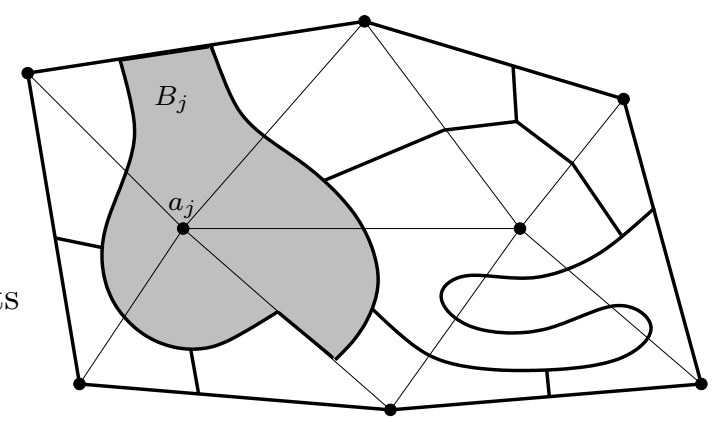

Abbildung 2.1: Duales Boxgitter

Allgemein heißt eine Menge $A$, die Bedingung (V2) erfüllt, d. h. deren Inneres ein LipschitzGebiet und die im Abschluß ihres Inneren enthalten ist, Lipschitz-Menge. Wegen $A \subseteq \overline{A^{\circ}}$ gilt für diese

$$
\partial A=\bar{A} \cap \overline{\Omega \backslash A} \subseteq \overline{A^{\circ}} \cap \overline{\Omega \backslash A^{\circ}}=\partial A^{\circ},
$$

also $\partial A=\partial A^{\circ}$. Dies rechtfertigt den Namen und erlaubt partielle Integration. 
Ist $\mathcal{B}_{h}=\left\{B_{1}, \ldots, B_{N_{h}}\right\}$ ein duales Boxgitter zu $\mathcal{T}_{h}$, so definieren wir die Finite-VolumenRäume

$$
\begin{aligned}
& P^{0}\left(\mathcal{B}_{h}\right):=\left\{v \in L^{2}(\Omega)|v|_{B} \in P_{0}(B) \text { für jedes } B \in \mathcal{B}_{h}\right\}, \\
& P_{0}^{0}\left(\mathcal{B}_{h}\right):=\left\{v \in P^{0}\left(\mathcal{B}_{h}\right)|v|_{B_{i}}=0 \text { für } i=N_{h, 0}+1, \ldots, N_{h}\right\} .
\end{aligned}
$$

Die charakteristischen Funktionen $\chi_{i}:=\chi_{B_{i}}$ (genauer: deren Klassen in $L^{2}(\Omega)$ ) bilden für $i=1, \ldots, N_{h}$, eine Basis von $P^{0}\left(\mathcal{B}_{h}\right)$ und für $i=1, \ldots, N_{h, 0}$ eine Basis von $P_{0}^{0}\left(\mathcal{B}_{h}\right)$, genannt die charakteristische Basis von $P^{0}\left(\mathcal{B}_{h}\right)$ bzw. $P_{0}^{0}\left(\mathcal{B}_{h}\right)$.

Bezeichnet $\left\{a_{1}, \ldots, a_{N_{h}}\right\}$ die Menge der Knoten der Triangulierung $\mathcal{T}_{h}$, so ist die Restriktion $\Psi:=\left.L_{h}\right|_{P^{1}\left(\mathcal{T}_{h}\right)}$ des Lumping-Operators

$$
L_{h}:\left\{\begin{aligned}
C(\bar{\Omega}) & \rightarrow P^{0}\left(\mathcal{B}_{h}\right) \\
v & \mapsto \sum_{j=1}^{N_{h}} v\left(a_{j}\right) \chi_{j}
\end{aligned}\right.
$$

ein (Vektorraum-)Isomorphismus zwischen $P^{1}\left(\mathcal{T}_{h}\right)$ und $P^{0}\left(\mathcal{B}_{h}\right)$ sowie $\left.\Psi\right|_{P_{0}^{1}\left(\mathcal{T}_{h}\right)}$ ein Isomorphismus zwischen $P_{0}^{1}\left(\mathcal{T}_{h}\right)$ und $P_{0}^{0}\left(\mathcal{B}_{h}\right)$ mit $\Psi\left(\varphi_{j}\right)=\chi_{j}$. Alternativ schreiben wir $\hat{v}$ anstatt $\Psi(v)$. Diese Abbildung $\Psi$ wird es uns später erlauben, eine Finite-Volumen-Diskretisierung als einen konformen verallgemeinerten Galerkin-Ansatz zu formulieren.

Für den Abstand eines Elements $v \in P^{1}\left(\mathcal{T}_{h}\right)$ zu seinem Bild $\hat{v} \in P^{0}\left(\mathcal{B}_{h}\right)$ in der $L^{2}$-Norm gilt (siehe [9], Lemma 4.2.15, und [35]):

Lemma 2.2.1 Es sei $\Omega \subseteq \mathbb{R}^{d}$ ein polyedrisches Lipschitz-Gebiet mit zulässiger Triangulierung $\mathcal{T}_{h}$. Ist $\mathcal{B}_{h}$ ein duales Boxgitter zu $\mathcal{T}_{h}$, so gilt

$$
\|v-\hat{v}\|_{0} \leqslant h|v|_{1}
$$

für jedes $v \in P^{1}\left(\mathcal{T}_{h}\right)$.

Bei der späteren Abschätzung der Konvektions-, Reaktions- und Quellterme in der FinitenVolumen-Diskretisierung gegen diese Terme in der Finiten-Element-Diskretisierung wird die Differenz $v-\hat{v}$ wesentlich eingehen.

Außerdem wird sich die folgende Abschätzung zwischen $\|\hat{v}\|_{0}$ und $\|v\|_{0}$ als nützlich erweisen, insbesondere bei den Abschätzungen zum stabilisierten Finiten-Volumen-Verfahren im Abschnitt 4.3.

Lemma 2.2.2 Es sei $\Omega \subseteq \mathbb{R}^{d}$ ein polyedrisches Lipschitz-Gebiet und $\mathcal{T}_{h}$ eine zulässige Triangulierungen darauf, die durch affine Transformation von einem Referenzelement erzeugt wird. Für jede Funktion $v \in P^{1}\left(\mathcal{T}_{h}\right)$ gilt dann

$$
\|\hat{v}\|_{0} \leqslant \sqrt{(d+1)(d+2)}\|v\|_{0} .
$$


Beweis. $\quad$ Es gilt einerseits

$$
\int_{\Omega} \hat{v}^{2} d x=\sum_{i \in N_{h, 0}} v_{i}^{2} \sum_{T \in \mathcal{T}_{h}}\left|B_{i} \cap T\right|=\sum_{T \in \mathcal{T}_{h}} \sum_{a_{i} \in T} v_{i}^{2}\left|B_{i} \cap T\right| \leqslant \sum_{T \in \mathcal{T}_{h}} \sum_{a_{i} \in T} v_{i}^{2}|T|
$$

und andererseits

$$
\int_{\Omega} v^{2} d x=\frac{1}{(d+1)(d+2)} \sum_{T \in \mathcal{T}_{h}}|T|\left(\sum_{a_{i} \in T} v_{i}^{2}+\left(\sum_{a_{i} \in T} v_{i}\right)^{2}\right)
$$

für Funktionen $v \in P^{1}\left(\mathcal{T}_{h}\right)$.

\subsection{Finite-Volumen-Diskretisierung}

Ein duales Boxgitter zerlegt das betrachtete Gebiet $\Omega$ in endlich viele Volumina. Es zeigt sich, daß im kontinuierlichen Fall jede solche Zerlegung auf eine Formulierung führt, die gleichwertig zur schwachen Formulierung (1.2) ist. Aus dieser äquivalenten Formulierung leiten wir schließlich unsere eigentliche Finite-Volumen-Diskretisierung ab. Dabei orientieren wir uns an der Technik für elliptische Probleme aus den Arbeiten [35, 9]. Für zeitabhängige nichtlineare Konvektions-Diffusions-Probleme siehe auch [22, 23].

Es sei $\mathcal{B}$ eine Zerlegung von $\Omega$ in endlich viele abgeschlossene Lipschitz-Mengen. Wegen der Voraussetzungen (1.4) existiert laut Produktregel (Satz A.2.1) für jedes $u \in V=H_{0}^{1}(\Omega)$ und $B \in \mathcal{B}$ die schwache Divergenz $\nabla \cdot(u b)$ in $L^{2}(B)$. Nach dem Spursatz A.2.4 existiert somit

$$
\int_{\partial B} u v b \cdot d s:=\left\langle\operatorname{ntr}_{\partial B} u b, \operatorname{tr}_{\partial B} v\right\rangle
$$

für alle $v \in V$.

Analog ist die Existenz von

$$
\int_{\partial B} v A \nabla u \cdot d s:=\left\langle\operatorname{ntr}_{\partial B} A \nabla u, \operatorname{tr}_{\partial B} v\right\rangle
$$

gewährleistet für

$$
u \in H_{0}^{1}(\Omega ; A \nabla):=\{u \in V \mid A \nabla u \in H(\operatorname{div} ; \Omega)\},
$$

$v \in V$ und $B \in \mathcal{B}$. Daher ist die Bilinearform

$$
a_{\mathcal{B}}(u, v):=\sum_{B \in \mathcal{B}}\left(\int_{B} \nabla v \cdot(A \nabla u-u b)+c u v d x-\int_{\partial B} v(A \nabla u-u b) \cdot d s\right)
$$

für alle $(u, v) \in H_{0}^{1}(\Omega ; A \nabla) \times V$ wohldefiniert. Das damit gebildete Variationsproblem:

Finde $u \in L^{2}\left(0, T ; H_{0}^{1}(\Omega ; A \nabla)\right)$ mit $u^{\prime} \in L^{2}\left(0, T ; V^{*}\right)$ und

$$
\left\{\begin{aligned}
\frac{d}{d t}(u(t), v)+a_{\mathcal{B}}(u(t), v) & =(f(t), v) \quad \text { für fast alle } t \in(0, T) \text { und alle } v \in V \\
u(0) & =u_{0} \in L^{2}(\Omega)
\end{aligned}\right.
$$


heißt schwache Formulierung von (1.2) bezüglich $\mathcal{B}$. Die Existenz einer eindeutig bestimmten Lösung wird durch die Äquivalenz zu (1.2) gewährleistet.

Lemma 2.3.1 Es sei $\Omega \subseteq \mathbb{R}^{d}$ ein beschränktes Lipschitz-Gebiet und $\mathcal{B}$ eine Zerlegung von $\Omega$ in endlich viele abgeschlossene Lipschitz-Mengen. Unter den Voraussetzungen (1.4) ist $u \in H^{1}(0, T ; V)$ genau dann eine Lösung von (1.2), wenn u Element von $L^{2}\left(0, T ; H_{0}^{1}(\Omega ; A \nabla)\right)$ ist und Lösung von (2.2) ist.

Beweis. $\quad$ Siehe Satz 4.1.2 in [9].

Wir werden nun noch die Konsistenzfehlerabschätzungen des nächsten Abschnitts durch eine angepaßte Bilinearform vorbereiten. Denn nutzt man die Bilinearform (2.1) zur räumlichen Finite-Volumen-Diskretisierung, so gelangt man zu Fehlerabschätzungen in der $L^{2}$-Norm, die um eine Ordnung schlechter sind als die bekannten Fehlerabschätzungen bei Finite-ElementDiskretisierungen mit $P_{1}$-Elementen, siehe [9], Abschnitt 4.3.4. Gleichwertige Fehlerabschätzungen ergeben sich jedoch, wenn man den Diffusionsterm von (2.1) modifiziert (vergl. ebenfalls [9], die Abschätzungen dort basieren auf [35]). Dazu werden die Komponenten von $A$ durch ihre Mittelwerte bezüglich $\mathcal{T}_{h}$ ersetzt.

Es sei $v \mapsto \bar{v}$ die orthogonale Projektion von $L^{2}(\Omega)$ auf $P^{0}\left(\mathcal{T}_{h}\right)$, d. h.

$$
(v, w)=(\bar{v}, w) \quad \text { für jedes } w \in P^{0}\left(\mathcal{T}_{h}\right) .
$$

Dann gilt für jedes $T \in \mathcal{T}_{h}$

$$
\left.\bar{v}\right|_{T^{\circ}}=p_{T}(v):=\frac{1}{\operatorname{vol}(T)} \int_{T} v(x) d x .
$$

$P_{T}$ ist Projektionsoperator $H^{1}(T) \rightarrow P_{0}(T)$, daher folgt für den Projektionsfehler (siehe etwa $[17])$

$$
\|v-\bar{v}\|_{p} \leqslant C_{P} h|v|_{1, p} \quad v \in H^{1}(\Omega), \quad p \in[1, \infty] .
$$

Nach diesen Anpassungen der Bilinearform lautet unsere Finite-Volumen-Diskretisierung zu $\mathcal{T}_{h}$ und $\mathcal{B}_{h}$ schließlich:

Finde $u_{h} \in H^{1}\left(0, T ; V_{h}\right), V_{h}=P_{0}^{1}\left(\mathcal{T}_{h}\right)$, so daß

$$
\left\{\begin{aligned}
\frac{d}{d t}\left(u_{h}(t), v\right)+a_{h}\left(u_{h}(t), v\right) & =\left\langle F_{h}(t), v\right\rangle \quad \text { für fast alle } t \in(0, T) \text { und alle } v \in V_{h} \\
u_{h}(0) & =u_{0, h} \in V_{h}
\end{aligned}\right.
$$

mit Bilinearform

$$
a_{h}(u, v):=\sum_{B \in \mathcal{B}_{h}}\left(\int_{B} c u \hat{v} d x-\int_{\partial B \backslash \partial \Omega} \hat{v} \bar{A} \nabla u \cdot d s+\int_{\partial B} u \hat{v} b \cdot d s\right)
$$

und Linearform

$$
\left\langle F_{h}(t), v\right\rangle:=\int_{\Omega} f(t) \hat{v} d x
$$

für $u, v \in P^{1}\left(\mathcal{T}_{h}\right)$. Dabei ist $\hat{v}=\Psi(v)$ und $\bar{A}=\left(\overline{a_{i j}}\right)_{i, j \in \underline{d}}$. 
Es wird sich zeigen, daß die $V_{h}$-Elliptizität und Stetigkeit von $a_{h}$ zumindest für hinreichend kleine $h>0$ aus der $V$-Elliptizität bzw. der Stetigkeit von $a$ laut (1.3) und der noch zu zeigenden Konsistenzfehlerabschätzung folgt.

Bemerkung 2.3.2 Um den Zusammenhang mit der klassischen Finite-Volumen-Technik im Fall von zweidimensionalen Problemen, also $\Omega \subset \mathbb{R}^{2}$, aufzuzeigen, betrachten wir zum einen die Bilinearform (2.5) für die Basisfunktionen $\left\{\chi_{B} \mid B \in \mathcal{B}_{h}\right\}$ von $P^{0}\left(\mathcal{B}_{h}\right)$ (vergl. Abschnitt 2.2). Für jedes $B \in \mathcal{B}_{h}$ und $\hat{v}=\chi_{B}$ reduziert sich die Bilinearform (2.5) auf ein Problem auf $B$ :

$$
a_{h}(u, v)=\int_{B} c u d x-\int_{\partial B \backslash \partial \Omega} \bar{A} \nabla u \cdot d s+\int_{\partial B} u b \cdot d s, \quad u \in P^{1}\left(\mathcal{T}_{h}\right) .
$$

Ferner sei die Diffusionsmatrix von der Form $A=\left(\varepsilon \delta_{i j}\right)_{i, j \in \underline{d}}$, und die Kontrollvolumina polygonal berandet. Sind $a_{i}$ und $a_{j}$ zwei benachbarte Knoten, $B_{i}$ und $B_{j}$ die zugehörigen Kontrollvolumina sowie $K$ eine Kante des gemeinsamen Randes $B_{i} \cap B_{j}$, so läßt sich der Diffusionsterm zum Kontrollvolumen $B_{i}$ durch

$$
\varepsilon \int_{K} \nabla u \cdot d s=\varepsilon \int_{K} \frac{\partial u}{\partial n_{i}} d s \leadsto \varepsilon \frac{u\left(a_{j}\right)-u\left(a_{i}\right)}{|K|}
$$

approximieren, wobei $n_{i}$ die äußere Normale an $B_{i}$ darstellt.

\subsection{Gleichgewichts- und Regularitätsbedingungen}

Weiterhin sei $\Omega \subseteq \mathbb{R}^{d}$ ein polyedrisches Lipschitz-Gebiet und $\mathcal{T}_{h}$ eine zulässige, exakte Triangulierung darauf. Ist $T=\operatorname{conv}\left(\left\{x_{T}^{(0)}, \ldots, x_{T}^{(d)}\right\}\right)$ ein reguläres Simplex aus $\mathcal{T}_{h}$ und $k \in d+1$, so bezeichne

$$
S_{k}(T):=\operatorname{conv}\left(\left\{x_{T}^{(j)} \mid j \in d+1 \backslash\{k\}\right\}\right),
$$

also das dem Eckpunkt $x_{T}^{(k)}$ gegenüberliegende $d$-1-Randsimplex von $T$. Abbildung 2.2 zeigt beispielhaft die Zuordnung von Randsimplizes und Eckpunkten.

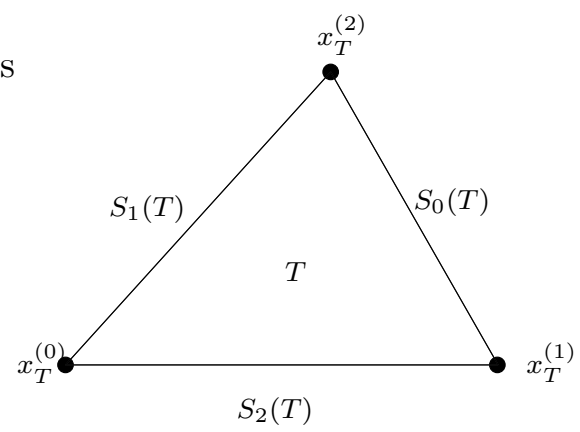

Abbildung 2.2: Die Randsimplizes $S_{k}(T)$ von $T$ 
$\mathrm{Zu}$ jedem Knoten $a_{j} \in T$ gibt es genau einen Index $i \in d+1$ mit $a_{j}=x_{T}^{(i)}$. Diesen bezeichnen wir mit $i(j, T)$. Desweiteren sei

$$
\begin{aligned}
S_{j, k}(T) & :=\quad B_{j} \cap S_{k}(T), \\
R_{j}(T) & :=\quad S_{j, i(j, T)}(T) \cup \overline{\left(\partial B_{j} \cap T^{\circ}\right)},
\end{aligned}
$$

falls $\mathcal{B}_{h}=\left\{B_{1}, \ldots, B_{N_{h}}\right\}$ ein duales Boxgitter zu $\mathcal{T}_{h}$ ist. In Abbildung 2.3 sind diese Teilmengen des Randes von $B_{j} \cap T$ exemplarisch dargestellt.

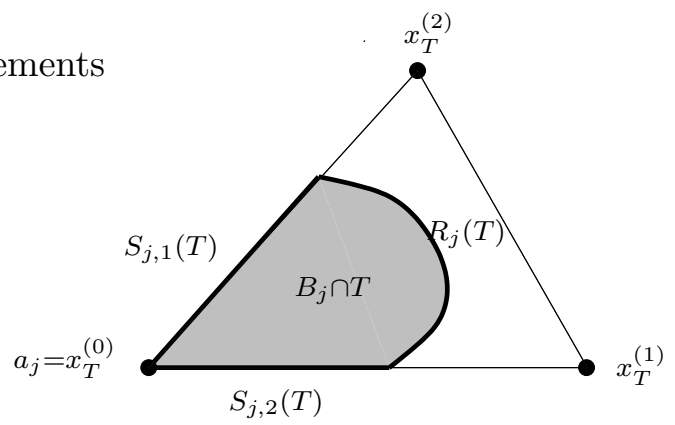

Abbildung 2.3: Der Rand von $B_{j} \cap T$

Wir kommen nun zu den angekündigten und durch obige Notation vorbereiteten Gleichgewichts- und Regularitätsbedingungen für ein duales Boxgitter $\mathcal{B}_{h} \mathrm{zu} \mathcal{T}_{h}$.

(G1) $\mathcal{B}_{h}$ erfüllt die erste Gleichgewichtsbedingung, falls

$$
\operatorname{vol}\left(B_{j} \cap T\right)=\frac{\operatorname{vol}(T)}{d+1}
$$

für jedes $j \in \underline{N_{h}}$ und jedes $T \in \mathcal{T}_{h}$ mit $a_{j} \in T$ gilt.

(G2) $\mathcal{B}_{h}$ erfüllt die zweite Gleichgewichtsbedingung, falls

$$
\operatorname{vol}\left(B_{j} \cap S_{k}(T)\right)=\frac{\operatorname{vol}\left(S_{k}(T)\right)}{d}
$$

für jedes $j \in \underline{N_{h}}$, jedes $T \in \mathcal{T}_{h}$ mit $a_{j} \in T$ und jedes $k \in d+1 \backslash i(j, T)$ gilt.

(R1) $\mathcal{B}_{h}$ erfüllt die erste Regularitätsbedingung, wenn $B_{j} \cap T$ eine Lipschitz-Menge ist für jedes $j \in N_{h}$ und jedes $T \in \mathcal{T}_{h}$ mit $a_{j} \in T$.

(R2) $\mathcal{B}_{h}$ erfüllt die zweite Regularitätsbedingung, wenn

$$
R_{j}(T) \cap S_{i(j, T)}=\emptyset
$$

für jedes $j \in \underline{N_{h}}$ und jedes $T \in \mathcal{T}_{h}$ mit $a_{j} \in T$ gilt. 
Die Bedingung (R2) fordert, daß der Rand des Volumens $B_{j}$ um einen Knoten $a_{j}$ keine der Kanten bzw. Randflächen schneidet, die in einer der $a_{j}$ umgebenden Simplizes diesem Knoten gegenüberliegen. Abgeschwächt kann man fordern, daß ein entsprechender Schnitt vom $(d-1)-\mathrm{Maß}$ Null ist.

Die Gleichgewichtsbedingung (G1) hat für Funktionen $v \in P^{1}\left(\mathcal{T}_{h}\right)$ die $L^{2}$-Orthogonalität der Differenz $v-\hat{v}$ zu $P^{0}\left(\mathcal{T}_{h}\right)$ zur Folge:

Lemma 2.4.1 Erfüllt $\mathcal{B}_{h}$ die Gleichgewichtsbedingung (G1), so gilt für je zwei Funktionen $u \in P^{0}\left(\mathcal{T}_{h}\right)$ und $v \in P^{1}\left(\mathcal{T}_{h}\right)$

$$
(u, v-\hat{v})=0 .
$$

Beweis. Wir zeigen die Behauptung für eine beliebige nodale Basisfunktion $\varphi_{j}$ von $P^{1}\left(\mathcal{T}_{h}\right)$. Bezeichnet $a_{j}$ den zugehörigen Knoten der Triangulierung, also $\varphi_{j}\left(a_{i}\right)=\delta_{i j}$, so gilt

$$
\int_{\Omega} u \varphi_{j} d x=\left.\sum_{\substack{T \in \mathcal{T}_{h} \\ a_{j} \in T}} u\right|_{T} \int_{T} \varphi_{j} d x=\left.\sum_{\substack{T \in \mathcal{T}_{h} \\ a_{j} \in T}} u\right|_{T} \frac{\operatorname{vol}(T)}{d+1}
$$

für jedes $u \in P^{0}\left(\mathcal{T}_{h}\right)$. Mit der ersten Gleichgewichtsbedingung (G1) erhält man daher

$$
\int_{\Omega} u \varphi_{j} d x=\left.\sum_{\substack{T \in \mathcal{T}_{h} \\ a_{j} \in T}} u\right|_{T} \operatorname{vol}\left(B_{j} \cap T\right)=\sum_{\substack{T \in \mathcal{T}_{h} \\ a_{j} \in T}} \int_{B_{j} \cap T} u d x=\int_{B_{j}} u d x=\int_{\Omega} u \chi_{j} d x
$$

Damit folgt die Behauptung für $\varphi_{j}$ wegen $\hat{\varphi}_{j}=\chi_{j}$.

Wie bereits erwähnt, finden in der Literatur nur sehr spezielle Boxgitter Anwendung. Und in der Tat, fordert man von einem Boxgitter mit polygonal berandeten Volumina die Erfüllung von (G1), (G2), (R1) und (R2), so gelangt man gerade zu einem Donald-Netz (siehe 2.5). Jedoch wird durch die Trennung der Definition des dualen Boxgitters von den Gleichgewichtsund Regularitätsbedingungen der Einfluß dieser Bedingungen auf die Konvergenzanalyse des Finite-Volumen-Verfahrens besonders deutlich. Eine polygonale Berandung ist dabei nicht entscheidend.

\subsection{Konstruktion von Donald-Netzen}

Die Abschätzungen des Abschnitts 3.1 werden auf Grundlage von Gleichgewichts- und Regularitätsanforderungen an das duale Boxgitter erzielt werden. An die Berandung der Kontrollvolumina wird dabei außer der Lipschitz-Stetigkeit keine weitere Voraussetzung gestellt werden. Für numerische Anwendungen jedoch möchte man möglichst einfach (d. h. polygonal) berandete Boxen haben.

In diesem Abschnitt werden wir eine Konstruktion von dualen Boxgittern angeben, die gerade durch Erfüllung jeder der genannten Anforderungen charakterisiert sind, also aus polygonal 
berandeten Boxen bestehen sowie den Bedingungen (G1), (G2), (R1) und (R3) genügen. Kurz gesprochen wird dazu jedem Knoten der Ausgangstriangulierung die Vereinigung der konvexen Hüllen der Schwerpunkte aller umliegenden Simplizes und Randsimplizes als Kontrollvolumen zugeordnet.

Für jedes $T=\operatorname{conv}\left(\left\{x_{T}^{(0)}, \ldots, x_{T}^{(d)}\right\}\right) \in \mathcal{T}_{h}$ einer zulässigen Triangulierung $\mathcal{T}_{h}$ bezeichne

$$
B_{i}(T):=\operatorname{conv}\left(\left\{\sigma(S) \mid S=T \text { oder } S \text { ist Randsimplex von } T \text { mit } x_{T}^{(i)} \in S\right\}\right) .
$$

Die Abbildung 2.4 zeigt ein solches Teilvolumen $B_{i}(T)$ und die Schwerpunkte, die dieses bestimmen.
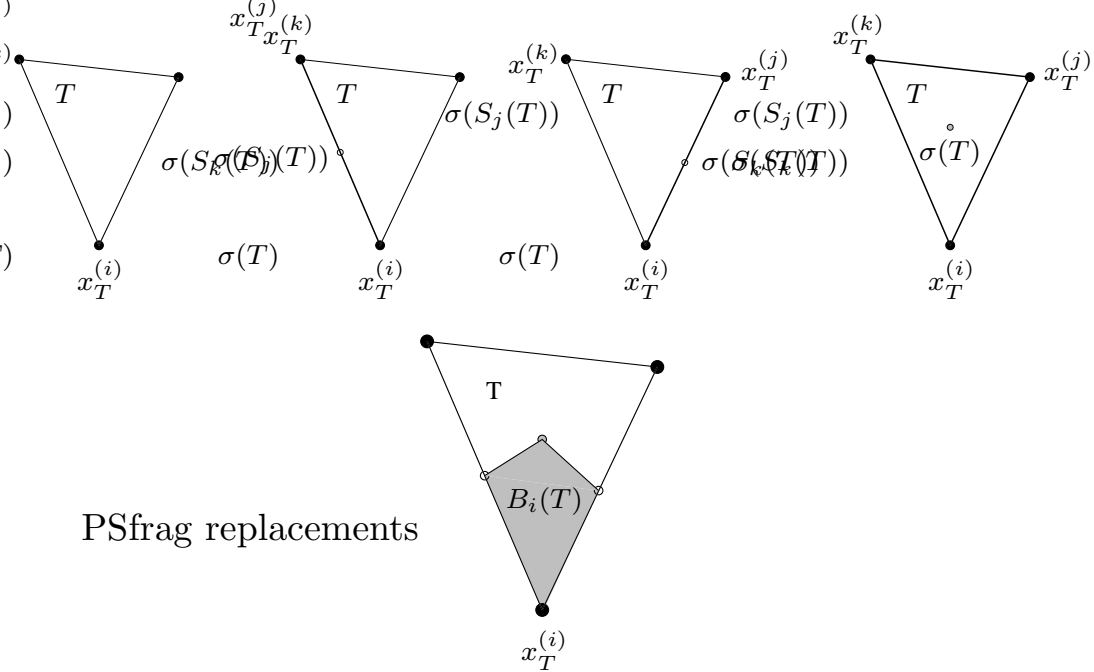

Abbildung 2.4: Schwerpunkte des Simplex T und der Randsimplizes, die $x_{T}^{(i)}$ enthalten (oben), Teilvolumen $B_{i}(T)$ (unten)

Aus diesen „Teilvolumina“ werden die eigentlichen Kontrollvolumina dual zu $\mathcal{T}_{h}$ im Sinne von (V1) - (V2) zusammengesetzt: Es sei $\mathcal{B}_{h}^{D}=\left\{B_{1}, \ldots, B_{N_{h}}\right\}$ das Mengensystem mit

$$
B_{j}:=\bigcup\left\{B_{i(j, T)}(T) \mid T \in \mathcal{T}_{h} \text { mit } a_{j} \in T\right\}, \quad j \in \underline{N_{h}} .
$$

$\mathcal{B}_{h}^{D}$ heißt dann Donald-Diagramm (oder auch Donald-Netz) zur Triangulierung $\mathcal{T}_{h}$. Abbildung 2.5 zeigt ein Beispiel.

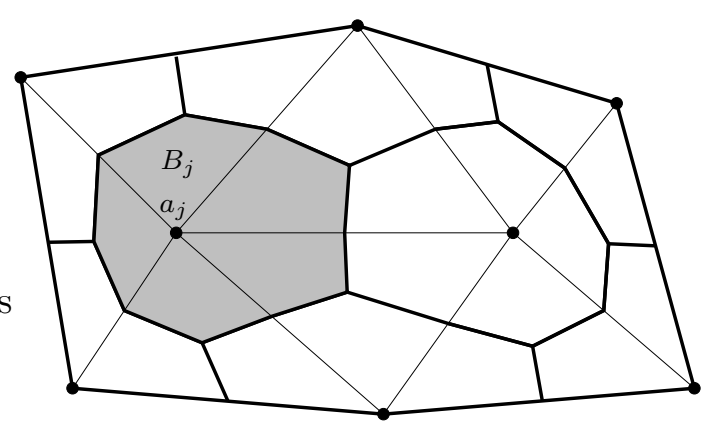

Abbildung 2.5: Donald Diagramm 
Für den Nachweis, daß $\mathcal{B}_{h}^{D}$ die Eigenschaften (G1), (G2), (R1) und (R2) trägt, erweist sich die folgende Darstellung durch baryzentrische Koordinaten als besonders günstig (zu Einzelheiten siehe [9], Abschnitt 4.4):

$$
B_{i}(T)=\left\{x=\sum_{j \in d+1} \lambda_{j} x_{T}^{(j)} \mid \sum \lambda_{j}=1, \lambda_{i} \geqslant \lambda_{j} \geqslant 0 \text { für } j \in d+1\right\} .
$$

$B_{i}(T)$ besteht also aus den Punkten $x \in \mathbb{R}^{d}$, für die $\lambda_{i}=\lambda_{i}(x)$ maxmimale baryzentrische Koordinate bezüglich $T$ ist.

In [59] wird für ein duales Boxgitter die Bezeichnung sekundäres Gitter benutzt. Die Boxen eines Donald-Diagramms werden dort wegen der Darstellung (2.9) baryzentrische duale Gebiete genannt.

Man beachte, daß die so konstruierten Kontrollvolumina im Gegensatz zu einigen anderen gebräuchlichen dualen Boxen (etwa eines Voronoi-Diagramms, also mit Hilfe von Mittelsenkrechten konstruierten Boxen) im allgemeinen nicht konvex sind. Man kommt allerdings völlig ohne Bedingungen an die Innenwinkel der Simplizes der Ausgangstriangulierung aus.

Schließlich bleiben für das Donald-Diagramm die gewünschten Gleichgewichts- und Regularitätsbedingungen nachzuweisen.

Satz 2.5.1 Es sei $\Omega \in \mathbb{R}^{d}$ ein polyedrisches Lipschitz-Gebiet und $\mathcal{T}_{h}$ eine zulässige Triangulierungen darauf. Dann ist das Donald-Diagramm ein duales Boxgitter zu $\mathcal{T}_{h}$, welches die Gleichgewichtsbedingungen (G1) und (G2) sowie die Regularitätsbedingungen (R1) und (R2) erfüllt.

Beweis. $\quad$ Satz 4.4.7 und Satz 4.4.9 in [9]. 


\section{Kapitel 3}

\section{Fehlerabschätzung zum Finite-Volumen-Verfahren}

Gegenstand dieses Kapitels sind Stabilitäts- und a-priori-Fehlerabschätzungen für das im vorausgegangenen Kapitel eingeführte Finite-Volumen-Verfahren. Es werden Abschätzungen sowohl für den semidiskreten als auch für den volldiskretisierten Fall gezeigt.

Dabei gehen wir so vor, daß wir im ersten Abschnitt eine Konsistenzfehlerabschätzung des Finite-Volumen-Verfahrens herleiten, die dann grundlegend in sämtliche Fehlerabschätzungen einfließt. Die aus der Literatur bekannten Ordnungen für a-priori-Fehlerabschätzungen im Fall von Finite-Element-Diskretisierung mit $P_{1}$-Elementen können auf diesem Wege erreicht werden.

\subsection{Konsistenzfehlerabschätzung}

Die im Abschnitt 2.3 eingeführte Finite-Volumen-Diskretisierung

Finde $u_{h} \in H^{1}\left(0, T ; V_{h}\right)$ mit

$$
\left\{\begin{aligned}
\frac{d}{d t}\left(u_{h}(t), v\right)+a_{h}\left(u_{h}(t), v\right) & =\left\langle F_{h}(t), v\right\rangle \quad \text { für fast alle } t \in(0, T) \text { und alle } v \in V_{h} \\
u_{h}(0) & =u_{0, h} \in V_{h}
\end{aligned}\right.
$$

wobei $V_{h}=P_{0}^{1}\left(\mathcal{T}_{h}\right)$,

$$
\begin{aligned}
a_{h}(u, v) & =\sum_{B \in \mathcal{B}_{h}}\left(\int_{B} c u \hat{v} d x-\int_{\partial B \backslash \partial \Omega} \hat{v} \bar{A} \nabla u \cdot d s+\int_{\partial B} u b \hat{v} \cdot d s\right), \\
\left\langle F_{h}(t), v\right\rangle & =\int_{\Omega} f(t) \hat{v} d x
\end{aligned}
$$

und $\hat{v}=\Psi(v)$ 
stellt dank des Isomorphismus $\Psi: V_{h}=P_{0}^{1}\left(\mathcal{T}_{h}\right) \rightarrow P_{0}^{0}\left(\mathcal{B}_{h}\right)$ (siehe Abschnitt 2.2) einen konformen verallgemeinerten Galerkin-Ansatz zum Problem (1.2) - (1.4) dar. Für die Differenz $e_{h}:=u-u_{h}$ zweier Lösungen $u$ und $u_{h}$ von (1.2) - (1.4) bzw. (3.1) - (3.3) läßt sich daher die Fehlergleichung

$$
\left\langle e_{h}^{\prime}(t), v\right\rangle+a\left(e_{h}(t), v\right)=F_{h}(t, v)-A_{h}(t, v)
$$

für jedes $v \in V_{h}$ formulieren, wobei

$$
\begin{aligned}
A_{h}(t, v) & :=a\left(u_{h}(t), v\right)-a_{h}\left(u_{h}(t), v\right), \\
F_{h}(t, v) & :=(f(t), v)-\left\langle F_{h}(t), v\right\rangle=(f(t), v-\hat{v}) .
\end{aligned}
$$

Die Differenz der beiden zugehörigen Bilinearformen $a$ und $a_{h}$ läßt sich wegen $\nabla\left(\left.\tilde{v}\right|_{B}\right)=0$ für jedes $B \in \mathcal{B}_{h}$ mit Hilfe der Greenschen Formel (siehe Folgerung A.2.5) schreiben als

$$
\begin{aligned}
a(u, v)-a_{h}(u, v)= & \int_{\Omega} \nabla v \cdot A \nabla u d x+\sum_{B \in \mathcal{B}_{h}} \int_{\partial B \backslash \partial \Omega} \hat{v} \bar{A} \nabla u \cdot d s \\
& +\int_{\Omega}(v-\hat{v}) \nabla \cdot(u b) d x+\int_{\Omega} c u(v-\hat{v}) d x, \quad u, v \in P^{1}\left(\mathcal{T}_{h}\right) .
\end{aligned}
$$

Um die Konsistenzfehler

$$
\left|a(u, v)-a_{h}(u, v)\right| \quad \text { und } \quad|(f(t), v-\hat{v})|
$$

abzuschätzen, greifen wir auf Abschätzungen der einzelnen auftretenden Terme zurück, wie sie in [9] gezeigt werden. Wir blicken zunächst auf die Differenz der Diffusionsterme.

Lemma 3.1.1 Es sei $\Omega \subseteq \mathbb{R}^{d}$ ein polyedrisches Lipschitz-Gebiet und $\mathcal{T}_{h}$ eine zulässige Triangulierung darauf. Ist $\mathcal{B}_{h}$ ein duales Boxgitter zu $\mathcal{T}_{h}$, das der Gleichgewichtsbedingung (G2) sowie den Regularitätsbedingungen (R1) und (R2) genügt, so gilt

$$
\int_{\Omega} \nabla v \cdot A \nabla u d x+\sum_{B \in \mathcal{B}_{h}} \int_{\partial B \backslash \partial \Omega} \hat{v} \bar{A} \nabla u \cdot d s=0
$$

für je zwei Funktionen $u, v \in P^{1}\left(\mathcal{T}_{h}\right)$. Insbesondere gilt

$$
\int_{\Omega} \nabla v \cdot A \nabla u d x+\sum_{B \in \mathcal{B}_{h}} \int_{\partial B} \hat{v} \bar{A} \nabla u \cdot d s=0
$$

für je zwei Funktionen $u, v \in P_{0}^{1}\left(\mathcal{T}_{h}\right)$.

Beweis. $\quad$ Vergl. Beweis zu Lemma 4.2.19 in [9].

Insbesondere zeigt dies, daß für reine Diffusionsprobleme die Finite-Volumen-Diskretisierung unter bestimmten Anforderungen an das duale Boxgitter mit der Finite-ElementDiskretisierung mit $P_{1}$-Elementen übereinstimmt. 
In der obigen Differenz zwischen $a$ und $a_{h}$ verbleiben für Funktionen $u, v \in P_{0}^{1}\left(\mathcal{T}_{h}\right)$ also nur die Beiträge der Konvektions- und Reaktionsterme. Mit $\nabla \cdot b \in L^{\infty}(\Omega)$ folgt daher

$$
\begin{aligned}
a(u, v)-a_{h}(u, v) & =\int_{\Omega}(v-\hat{v}) \nabla \cdot(u b) d x+\int_{\Omega} c u(v-\hat{v}) d x \\
& =\int_{\Omega}(v-\hat{v}) b \cdot \nabla u d x+\int_{\Omega}(\nabla \cdot b+c) u(v-\hat{v}) d x, \quad u, v \in P_{0}^{1}\left(\mathcal{T}_{h}\right) .
\end{aligned}
$$

Nun gilt aufgrund von Lemma 2.2.1

$$
|(u, v-\hat{v})| \leqslant h|| u \|_{0}|v|_{1}
$$

für beliebige Funktionen $u \in L^{2}(\Omega), v \in P^{1}\left(\mathcal{T}_{h}\right)$. Ist $u$ zudem $H^{1}(\Omega)$-regulär und genügt das duale Boxgitter $\mathcal{B}_{h}$ der Gleichgewichtsbedingung (G1), so folgt wegen (2.8) und (2.3) sogar die $O\left(h^{2}\right)$-Ungleichung

$$
|(u, v-\hat{v})| \leqslant h|| u-\bar{u} \|_{0}|v|_{1} \leqslant C_{P} h^{2}|u|_{1}|v|_{1} .
$$

Diese Abschätzungen bilden die Grundlage für die Abschätzungen der Konvektions-, Reaktions- und Quellterme.

Lemma 3.1.2 Es sei $\Omega \subseteq \mathbb{R}^{d}$ ein polyedrisches Lipschitz-Gebiet und $\mathcal{T}_{h}$ eine zulässige Triangulierung darauf sowie $\mathcal{B}_{h}$ ein duales Boxgitter $z u \mathcal{T}_{h}$. Für Funktionen $b \in L^{\infty}(\Omega)^{d}$ mit schwacher Divergenz $\nabla \cdot b \in L^{\infty}(\Omega), c \in L^{\infty}(\Omega), f \in L^{2}(\Omega)$ gelten dann die Abschätzungen

$$
\begin{aligned}
\left|\int_{\Omega}(v-\hat{v}) b \cdot \nabla u d x\right| & \leqslant h\|b\|_{\infty}|u|_{1}|v|_{1}, \\
\left|\int_{\Omega}(\nabla \cdot b+c) u(v-\hat{v}) d x\right| & \leqslant h\|\nabla \cdot b+c\|_{\infty}\|u\|_{0}|v|_{1}, \\
\left|\int_{\Omega} f(v-\hat{v}) d x\right| & \leqslant h\|f\|_{0}|v|_{1}
\end{aligned}
$$

für je zwei Funktionen $u, v \in P^{1}\left(\mathcal{T}_{h}\right)$. Erfüllt $\mathcal{B}_{h}$ zudem die Gleichgewichtsbedingung (G1), so erhält man für je zwei Funktionen $u, v \in P^{1}\left(\mathcal{T}_{h}\right)$

$$
\left|\int_{\Omega}(v-\hat{v}) b \cdot \nabla u d x\right| \leqslant C_{P} h^{2} \max _{i \in \underline{d}}\left\|b_{i}\right\|_{1, \infty}|u|_{1}|v|_{1},
$$

falls $b \in H^{1, \infty}(\Omega)^{d}$,

$$
\left|\int_{\Omega}(\nabla \cdot b+c) u(v-\hat{v}) d x\right| \leqslant C_{P} h^{2}\|\nabla \cdot b+c\|_{1, \infty}\|u\|_{1}|v|_{1},
$$

falls $\nabla \cdot b \in H^{1, \infty}(\Omega)$ und $c \in H^{1, \infty}(\Omega)$,

$$
\left|\int_{\Omega} f(v-\hat{v}) d x\right| \leqslant C_{P} h^{2}\|f\|_{1}|v|_{1}
$$

falls $f \in H^{1}(\Omega)$. Dabei ist $C_{P}>0$ eine Konstante, die weder von $h$ noch von $b, c$ oder $f$ abhängt. 
Beweis. $\quad$ Vergl. Beweise der Lemmata 4.2.21 - 4.2.23 in [9].

Zusammenfassend erhält man für den Fehler beim Übergang von der Bilinearform a zur Bilinearform $a_{h}$ die Abschätzung

$$
\left|a(u, v)-a_{h}(u, v)\right| \leqslant h\left(\|b\|_{\infty}|u|_{1}+\|\nabla \cdot b+c\|_{\infty}\|u\|_{0}\right)|v|_{1}
$$

und unter den jeweiligen stärkeren Bedingungen an $b, c$ und $f$ sowie $\mathcal{B}_{h}$

$$
\left|a(u, v)-a_{h}(u, v)\right| \leqslant C_{P} h^{2}\left(\max _{i \in \underline{d}}\left\|b_{i}\right\|_{1, \infty}+\|\nabla \cdot b+c\|_{1, \infty}\right)|u|_{1}|v|_{1}
$$

für je zwei Funktionen $u, v \in P^{1}\left(\mathcal{T}_{h}\right)$.

Im Abschnitt 1.1 wurde die Norm $\|\cdot\|_{\varepsilon}$ mit

$$
\|v\|_{\varepsilon}^{2}=\left\|A^{\frac{1}{2}} \nabla v\right\|_{0}^{2}+\|\sqrt{q} v\|_{0}^{2}
$$

für Funktionen $v \in H^{1}(\Omega)$ eingeführt, wobei

$$
\left\|A^{\frac{1}{2}} \nabla v\right\|_{0}^{2}:=\int_{\Omega} \nabla v \cdot A \nabla v d x, \quad q:=\frac{1}{2} \nabla \cdot b+c
$$

gesetzt und (1.8) angenommen wurde. Als Resultate bezüglich dieser problemangepaßten Norm halten wir fest

Lemma 3.1.3 Es seien die Voraussetzungen des Lemmas 3.1 .1 erfüllt. Für die Koeffizienten der Bilinearform a gemäß (1.3) gelte (1.4) und (1.8). Dann erhält man für das FiniteVolumen-Problem (3.1) - (3.3) die Konsistenzfehlerabschätzungen

$$
\left|a(u, v)-a_{h}(u, v)\right| \leqslant h C_{K}\|u\|_{\varepsilon}\|v\|_{\varepsilon}
$$

mit der Konstante $C_{K}:=\frac{\|b\|_{\infty}}{\varepsilon}+\frac{\|\nabla \cdot b+c\|_{\infty}}{\sqrt{\varepsilon \eta}}$ sowie

$$
|(f(t), v-\hat{v})| \leqslant h \frac{\|f(t)\|_{0}}{\sqrt{\varepsilon}}\|v\|_{\varepsilon}
$$

für je zwei Funktionen $u, v \in P^{1}\left(\mathcal{T}_{h}\right)$ und fast jedes $t \in(0, T)$. Sind zudem die verschärften Voraussetzungen von Lemma 3.1.2 erfüllt, so gilt

$$
\left|a(u, v)-a_{h}(u, v)\right| \leqslant h^{2} \tilde{C}_{K}\|u\|_{\varepsilon}\|v\|_{\varepsilon}
$$

mit der Konstante $\tilde{C}_{K}:=\frac{C_{P}}{\varepsilon}\left(\max _{i \in \underline{d}}\left\|b_{i}\right\|_{1, \infty}+\|\nabla \cdot b+c\|_{1, \infty}\right)$ sowie

$$
|(f(t), v-\hat{v})| \leqslant h^{2} \frac{C_{P}}{\sqrt{\varepsilon}}\|f(t)\|_{1}\|v\|_{\varepsilon}
$$


Beweis. $\quad$ Die Abschätzung (3.5) läßt sich mit Blick auf (1.8) wie folgt weiterführen

$$
\begin{aligned}
\left|a(u, v)-a_{h}(u, v)\right| & \leqslant h\left(\|b\|_{\infty}|u|_{1}+\|\nabla \cdot b+c\|_{\infty}\|u\|_{0}\right)|v|_{1} \\
& \leqslant h\left(\frac{\|b\|_{\infty}}{\varepsilon}\left\|A^{\frac{1}{2}} \nabla u\right\|_{0}+\frac{\|\nabla \cdot b+c\|_{\infty}}{\sqrt{\varepsilon \eta}}\|\sqrt{q} u\|_{0}\right)\left\|A^{\frac{1}{2}} \nabla v\right\|_{0} \\
& \leqslant h C_{K}\|u\|_{\varepsilon}\left\|A^{\frac{1}{2}} \nabla v\right\|_{0} .
\end{aligned}
$$

Für $|(f(t), v-\hat{v})|$ erhält man mit Lemma 3.1.2 und mit (1.8)

$$
|(f(t), v-\hat{v})| \leqslant h\|f(t)\|_{0}|v|_{1} \leqslant h \frac{\|f(t)\|_{0}}{\sqrt{\varepsilon}}\left\|A^{\frac{1}{2}} \nabla v\right\|_{0} .
$$

Analog ergeben sich unter den verschärften Voraussetzungen von Lemma 3.1.2 mit der Abschätzung (3.6) die letzten beiden Ungleichungen des Lemmas.

Bemerkung 3.1.4 Bisher wurden ausschließlich Probleme mit homogenen DirichletRandbedingungen betrachtet. Randintegrale über Gebietsrandabschnitte $\Gamma \subseteq \partial \Omega$ spielten daher keine Rolle. Es lassen sich jedoch die Abschätzungen

$$
\left|\int_{\Gamma} g(v-\hat{v}) d s\right| \leqslant C_{d} h^{\frac{3}{2}}\|g\|_{1, \Gamma}\|v\|_{1}, \quad\left|\int_{\Gamma} g u(v-\hat{v}) d s\right| \leqslant C_{d} h\|g\|_{1, \infty, \Gamma}\|u\|_{1}\|v\|_{1}
$$

und

$$
\left|\int_{\Gamma} g(u-\hat{u})(v-\hat{v}) d s\right| \leqslant C_{d} h\|g\|_{0, \infty, \Gamma}\|u\|_{1}\|v\|_{1}
$$

zeigen für Funktionen $u, v \in P^{1}\left(\mathcal{T}_{h}\right)$ und hinreichend glatte Funktion $g$ auf $\Gamma$ (siehe [9], Abschnitt 4.2.6). Dabei ist $C_{d}$ eine Konstante, die von der Raumdimension $d$, nicht jedoch von $h$ abhängt. Randintegrale der ersten Form treten beispielsweise bei Finite-VolumenDiskretisierungen entsprechend (3.1)-(3.3) mit inhomogenen Neumann-Randbedingungen auf, etwa $\operatorname{ntr}_{\Gamma}(A \nabla u(t)-u(t) b)=g$ mit hinreichend glatter Funktion $g$ auf dem Randabschnitt $\Gamma$. In solchen Fällen läßt sich bei Konsistenzfehlerabschätzung analog zu denen dieses Abschnitts daher teilweise nur die Ordnung $O\left(h^{\frac{3}{2}}\right)$ erzielen.

\subsection{Eindeutige Lösbarkeit und Stabilitätsabschätzungen}

Wir sind jetzt in der Lage, Aussagen zur Lösbarkeit des Problems (3.1) - (3.3) zu machen und Stabilitätsabschätzungen für die Bilinearform $a_{h}$ anzugeben, jeweils in Abhängigkeit von der Bilinearform $a$ gemäß (1.3).

Bei gegebener Stetigkeit und Koerzivität von $a$ bezüglich $H^{1}(\Omega)$ mit Konstanten $M>0$ bzw. $\alpha>0$ ergibt sich als Folgerung aus (3.5) sofort zum einen

$$
\left|a_{h}(u, v)\right| \leqslant|a(u, v)|+\left|a(u, v)-a_{h}(u, v)\right| \leqslant\left(M+C_{\infty} h\right)\|u\|_{1}\|v\|_{1}
$$


und zum anderen

$$
a_{h}(v, v) \geqslant a(v, v)-\left|a(v, v)-a_{h}(v, v)\right| \geqslant\left(\alpha-C_{\infty} h\right)\|v\|_{1}^{2}
$$

für alle $u, v \in P^{1}\left(\mathcal{T}_{h}\right)$, wobei abkürzend $C_{\infty}:=\|b\|_{\infty}+\|\nabla \cdot b+c\|_{\infty}$ benutzt wurde. Die Bilinearform (3.2) der Finite-Volumen-Diskretisierung ist also stetig und zumindest für $h<\frac{\alpha}{C_{\infty}}$ auch $P^{1}\left(\mathcal{T}_{h}\right)$-koerziv.

Damit ist Satz 1.2.1 anwendbar, und man erhält die folgende Aussage zur eindeutigen Lösbarkeit des Finite-Volumen-Problems.

Satz 3.2.1 Die durch (1.3) definierte Bilinearform erfülle (E2) mit $V=H_{0}^{1}(\Omega)$, und für $A$, $b, c, f$ gelte (1.4). Zudem sei (TR) vorausgesetzt, und zu jeder Triangulierung $\mathcal{T}_{h}, h>0$, sei $\mathcal{B}_{h}$ ein duales Boxgitter, welches den Bedingungen (G2), (R1) und (R2) genügt. Dann gibt es ein $h_{0}>0$, so daß für jedes $h \in\left(0, h_{0}\right)$ das semidiskrete Problem (3.1)-(3.3) genau eine Lösung $u_{h} \in H^{1}\left(0, T ; P_{0}^{1}\left(\mathcal{T}_{h}\right)\right)$ besitzt.

Wir geben nun noch Abschätzungen zur Stetigkeit und Koerzivität der Finite-VolumenBilinearform $a_{h}$ bezüglich der Norm $\|\cdot\|_{\varepsilon}$ an. Dazu sei (1.8) vorausgesetzt.

Zunächst gilt

$$
a(u, v)=\int_{\Omega} \nabla v \cdot A \nabla u+\left(\frac{1}{2} \nabla \cdot b+c\right) u v d x+\frac{1}{2} \int_{\Omega} v b \cdot \nabla u-u b \cdot \nabla v d x
$$

für $u, v \in H_{0}^{1}(\Omega)$ nach Gleichung (1.7). Daher ist die Koerzivität von $a$, nämlich

$$
a(v, v) \geqslant\|v\|_{\varepsilon}^{2} \quad \text { für alle } v \in H_{0}^{1}(\Omega),
$$

unmittelbar ablesbar. Die Stetigkeit von $a$ bezüglich der Norm $\|\cdot\|_{\varepsilon}$ folgt unter Ausnutzung von (1.10) und (1.8), denn es ist

$$
\begin{aligned}
|a(u, v)| \leqslant & \left|\int_{\Omega} \nabla v \cdot A \nabla u d x\right|+\left|\int_{\Omega} q u v d x\right|+\frac{1}{2}\left|\int_{\Omega} v b \cdot \nabla u d x\right|+\frac{1}{2}\left|\int_{\Omega} u b \cdot \nabla v d x\right| \\
\leqslant & \left\|A^{\frac{1}{2}} \nabla u\right\|_{0}\left\|A^{\frac{1}{2}} \nabla v\right\|_{0}+\|\sqrt{q} u\|_{0}\|\sqrt{q} v\|_{0} \\
& +\frac{1}{2}\|b\|_{\infty}\left(\|v\|_{0}\|\nabla u\|_{0}+\|u\|_{0}\|\nabla v\|_{0}\right) \\
\leqslant & \left\|A^{\frac{1}{2}} \nabla u\right\|_{0}\left\|A^{\frac{1}{2}} \nabla v\right\|_{0}+\|\sqrt{q} u\|_{0}\|\sqrt{q} v\|_{0} \\
& +\frac{1}{2} \frac{\|b\|_{\infty}}{\sqrt{\varepsilon \eta}}\left(\|\sqrt{q} v\|_{0}\left\|A^{\frac{1}{2}} \nabla u\right\|_{0}+\|\sqrt{q} u\|_{0}\left\|A^{\frac{1}{2}} \nabla v\right\|_{0}\right) \\
\leqslant & M_{\|\cdot\|_{\varepsilon}}\|u\|_{\varepsilon}\|v\|_{\varepsilon}
\end{aligned}
$$

mit $M_{\|\cdot\|_{\varepsilon}}:=1+\frac{\|b\|_{\infty}}{2 \sqrt{\varepsilon \eta}}$. Für die Bilinearform $a_{h}$ läßt sich daher mit Lemma 3.1.3 ableiten

$$
a_{h}(v, v) \geqslant a(v, v)-\left|a(v, v)-a_{h}(v, v)\right| \geqslant\left(1-C_{K} h\right)\|v\|_{\varepsilon}^{2}
$$

sowie

$$
\left|a_{h}(u, v)\right| \leqslant|a(u, v)|+\left|a(u, v)-a_{h}(u, v)\right| \leqslant\left(M_{\|\cdot\|_{\varepsilon}}+C_{K} h\right)\|u\|_{\varepsilon}\|v\|_{\varepsilon}
$$

für alle $u, v \in P_{0}^{1}\left(\mathcal{T}_{h}\right)$.

Wir fassen zusammen: 
Lemma 3.2.2 Unter den Voraussetzungen des Lemmas 3.1.1 sowie den Voraussetzungen (1.4) und (1.8) genügt die Finite-Volumen-Bilinearform (3.2) den Abschätzungen

$$
\left|a_{h}(u, v)\right| \leqslant\left(M_{\|\cdot\|_{\varepsilon}}+C_{K} h\right)\|u\|_{\varepsilon}\|v\|_{\varepsilon}
$$

und

$$
a_{h}(v, v) \geqslant\left(1-C_{K} h\right)\|v\|_{\varepsilon}^{2}
$$

für alle $u, v \in P_{0}^{1}\left(\mathcal{T}_{h}\right)$ mit den Konstanten $C_{K}=\frac{\|b\|_{\infty}}{\varepsilon}+\frac{\|\nabla \cdot b+c\|_{\infty}}{\sqrt{\varepsilon \eta}}$ und $M_{\|\cdot\|_{\varepsilon}}=1+\frac{\|b\|_{\infty}}{2 \sqrt{\varepsilon \eta}}$.

Die Koerzivität von $a_{h}$ bezüglich der Norm $\|\cdot\|_{\varepsilon}$ ist bei diesem Vorgehen nur unterhalb von $h_{0}:=\frac{1}{C_{K}}=\left(\frac{\|b\|_{\infty}}{\varepsilon}+\frac{\|\nabla \cdot b+c\|_{\infty}}{\sqrt{\varepsilon \eta}}\right)^{-1}$ gewährleistet, also in Abhängigkeit von der Stärke der Diffusion $\varepsilon$. Im Kapitel 4 werden wir hingegen eine stabilisierte Finite-Volumen-Diskretisierung einführen, bei der die Koerzivität der zugehörigen Bilinearform unabhängig von $\varepsilon$ gegeben ist.

\subsection{A-priori-Fehlerabschätzungen zum semidiskreten Pro- blem}

Die Existenz einer eindeutigen Lösung zum finite-volumen-diskretisierten Problem (3.1) (3.3) wurde durch Satz 3.2.1 für hinreichend kleines $h>0$ gezeigt. Da (3.1) - (3.3) eine Diskretisierung nur im Raum darstellt, ist eine solche Lösung kontinuierlich in der Zeit. Ziel dieses Abschnitts ist der Nachweis, daß diese Diskretisierung die Lösung des Ausgangsproblems $(1.2)$ - (1.4) bei geeignetem $h$ stabil bzw. beliebig gut approximiert.

In den nachfolgenden Beweisen wird vom Ritz-Operator Gebrauch gemacht. Deshalb seien hier einige grundlegende Eigenschaften aufgeführt (vergl. etwa [61] und [47]).

Lemma 3.3.1 Es sei a eine Bilinearform gemäß (1.3), deren Koeffizienten den Bedingungen (1.4) und (1.8) genügen. Zudem sei $\left(V_{h}\right)_{h>0}$ eine abzählbare Familie endlich-dimensionaler Unterräume von $V=H_{0}^{1}(\Omega)$. Dann gibt es zu jedem $u \in V$ und jedem $h>0$ ein eindeutig bestimmtes $u_{h} \in V_{h}$ mit

$$
a\left(u_{h}, v\right)=a(u, v) \quad \text { für jedes } v \in V_{h} .
$$

Für die damit definierte Abbildung $R:(h, u) \mapsto u_{h}=: R_{h} u$, genannt Ritz-Operator, gilt:

- Zu jedem $h>0$ ist $R(h, \cdot)=R_{h}$ bezüglich $\|\cdot\|_{\varepsilon}$ stetiger Projektionsoperator auf $V$, der mit dem Zeitableitungsoperator $\frac{d}{d t}$ kommutiert.

- Es existiert eine von $h$ unabhängige Konstante $\tilde{M}>0$, so daß

$\left\|u-R_{h} u\right\|_{\varepsilon} \leqslant \tilde{M} \inf _{v \in V_{h}}\|u-v\|_{1}$

für jedes $u \in V$. 
Ist zudem für jedes $r \in L^{2}(\Omega)$ die Lösung $u^{*}$ des adjungierten Problems

$$
a\left(v, u^{*}\right)=(r, v), \quad v \in V,
$$

$H^{2}(\Omega)$-regulär, und ist (TR) vorausgesetzt, so gilt mit $V_{h}=P^{k}\left(\mathcal{T}_{h}\right)$

$$
\left\|u-R_{h} u\right\|_{0}+h\left\|u-R_{h} u\right\|_{\varepsilon} \leqslant C_{R} h^{k+1}|u|_{k+1} \quad \text { für alle } u \in H^{k+1}(\Omega)
$$

mit einer von $h$ unabhängigen Konstante $C_{R}>0$.

Beweis. Aufgrund der Koerzivität und Stetigkeit von $a$ bezüglich der Norm $\|\cdot\|_{\varepsilon}$ bzw. (3.13) folgt

$$
\left\|R_{h} u\right\|_{\varepsilon}^{2} \leqslant a\left(R_{h} u, R_{h} u\right)=a\left(u, R_{h} u\right) \leqslant M_{\|\cdot\|_{\varepsilon}}\left\|R_{h} u\right\|_{\varepsilon}\|u\|_{\varepsilon},
$$

also die Stetigkeit von $R_{h}$ bezüglich $\|\cdot\|_{\varepsilon}$.

Desweiteren folgt aus der Ungleichung (3.12) die Beschränktheit von $a$ bezüglich $\|\cdot\|_{\varepsilon}$ in der ersten und bezüglich $\|\cdot\|_{1}$ in der zweiten Komponente.

$$
\begin{aligned}
|a(u, v)| \leqslant & \left\|A^{\frac{1}{2}} \nabla u\right\|_{0} C_{A}|v|_{1}+\|\sqrt{q} u\|_{0}\|\sqrt{q}\|_{\infty}\|v\|_{0} \\
& +\frac{1}{2}\|b\|_{\infty}\left(\frac{1}{\sqrt{\varepsilon}}\|v\|_{0}\left\|A^{\frac{1}{2}} \nabla u\right\|_{0}+\frac{1}{\sqrt{\eta}}\|\sqrt{q} u\|_{0}|v|_{1}\right) \\
\leqslant & \left(\left\|A^{\frac{1}{2}} \nabla u\right\|_{0}^{2}+\|\sqrt{q} u\|_{0}^{2}\right)^{\frac{1}{2}}\left(C_{A}|v|_{1}^{2}+\|\sqrt{q}\|_{\infty}\|v\|_{0}^{2}\right)^{\frac{1}{2}} \\
& +\frac{1}{2}\|b\|_{\infty}\left(\frac{1}{\varepsilon}\left\|A^{\frac{1}{2}} \nabla u\right\|_{0}^{2}+\frac{1}{\eta}\|\sqrt{q} u\|_{0}^{2}\right)^{\frac{1}{2}}\left(|v|_{1}^{2}+\|v\|_{0}^{2}\right)^{\frac{1}{2}} \\
\leqslant & \tilde{M}\|u\|_{\varepsilon}\|v\|_{1}
\end{aligned}
$$

$\operatorname{mit} \tilde{M}:=\max \left\{\sqrt{C_{A}}, \sqrt{\|\sqrt{q}\|_{\infty}}\right\}+\frac{1}{2}\|b\|_{\infty} \max \left\{\frac{1}{\sqrt{\varepsilon}}, \frac{1}{\sqrt{\eta}}\right\}, C_{A}:=d \max _{i, j \in \underline{d}}\left\|a_{i j}\right\|_{\infty}$. Daher ist

$$
\left\|u-R_{h} u\right\|_{\varepsilon}^{2} \leqslant a\left(u-R_{h} u, u-R_{h} u\right)=a\left(u-R_{h} u, u-v\right) \leqslant \tilde{M}\left\|u-R_{h} u\right\|_{\varepsilon}\|u-v\|_{1},
$$

d. h. $\left\|u-R_{h} u\right\|_{\varepsilon} \leqslant \tilde{M} \inf _{v \in V_{h}}\|u-v\|_{1}$ für $u \in V$. Nun gilt für jedes $u \in V, T \in \mathcal{T}_{h}$

$$
\inf _{v \in V_{h}}\|u-v\|_{1} \leqslant C h^{k}|u|_{k+1} \quad \text { für alle } u \in H^{k+1}(\Omega)
$$

mit einer Konstante $C>0$, die vom gewählten Refenrenzelement der Triangulierung und von der Dimension $d$ abhängt, nicht jedoch von $h$ (siehe etwa [47]). Damit erhält man

$$
\left\|u-R_{h} u\right\|_{\varepsilon} \leqslant \tilde{M} C h^{k}|u|_{k+1} \quad \text { für alle } u \in H^{k+1}(\Omega) .
$$

Für die noch fehlende Abschätzung von $\left\|u-R_{h} u\right\|_{0}$ siehe [57], Abschnitt 11.2. An diesem Punkt wird die $H^{2}(\Omega)$-Regularität von $u^{*}$ benötigt.

Desweiteren geben wir eine exponentiell gewichtete a-priori-Abschätzung für die Lösung $u_{h}$ zum Finite-Volumen-Problem (3.1) - (3.3) und eine a-priori-Abschätzung für deren Zeitableitung $u_{h}^{\prime}$ an. 
Lemma 3.3.2 Es seien die Voraussetzungen der Lemmata 2.2.2 und 3.1.1 sowie (1.4) und (1.8) erfüllt. Ferner gelte $h<\frac{1}{C_{K}}$. Dann genügt eine Lösung $u_{h} \in H^{1}\left(0, T ; V_{h}\right)$ des Problems (3.1) - (3.3) für fast alle $t \in(0, T)$ und alle $\lambda \in\left[0, \eta\left(1-C_{K} h\right)\right)$ den Abschätzungen

$$
\begin{aligned}
\left\|u_{h}(t)\right\|_{0}^{2}+\left(1-C_{K} h-\frac{\lambda}{\eta}\right) & \int_{0}^{t} e^{2 \lambda(s-t)}\left\|u_{h}(s)\right\|_{\varepsilon}^{2} d s \\
& \leqslant e^{-2 \lambda t}\left\|u_{0, h}\right\|_{0}^{2}+\frac{C_{d}}{\eta\left(1-C_{K} h\right)-\lambda} \int_{0}^{t} e^{2 \lambda(s-t)}\|f(s)\|_{0}^{2} d s
\end{aligned}
$$

und

$$
\begin{aligned}
\left\|A^{\frac{1}{2}} \nabla u_{h}(t)\right\|_{0}^{2}+\int_{0}^{t}\left\|u_{h}^{\prime}(s)\right\|_{0}^{2} d s \leqslant & \left\|A^{\frac{1}{2}} \nabla u_{0, h}\right\|_{0}^{2}+2 C_{d} \int_{0}^{t}\|f(s)\|_{0}^{2} d s \\
& +4 \max \left\{\frac{1}{\varepsilon}, \frac{1}{\eta}\right\} C_{d}\left(\|\nabla \cdot b\|_{\infty}^{2}+\|b\|_{\infty}^{2}+\|c\|_{\infty}^{2}\right) \int_{0}^{t}\left\|u_{h}(s)\right\|_{\varepsilon}^{2} d s .
\end{aligned}
$$

mit $C_{d}:=(d+1)(d+2)$.

Beweis. Wir machen uns wie in der Bemerkung 1.1.4 ein transformiertes Problem zu Nutze. Die Funktion $u_{h, \lambda}$ mit $u_{h, \lambda}(t):=e^{\lambda t} u_{h}(t)$ löst das Problem

$$
\left\{\begin{aligned}
\frac{d}{d t}\left(u_{h, \lambda}(t), v\right)+a_{h, \lambda}\left(u_{h, \lambda}(t), v\right) & =e^{\lambda t}\left\langle F_{h}(t), v\right\rangle \quad \text { für fast alle } t \in(0, T) \text { und alle } v \in V_{h} \\
u_{h, \lambda}(0) & =u_{0, h},
\end{aligned}\right.
$$

wobei $a_{h, \lambda}(u, v):=a_{h}(u, v)+\lambda(u, v)$.

Nach Lemma 3.2.2 und (1.8) gilt

$$
a_{h, \lambda}(v, v) \geqslant\left(1-C_{K} h\right)\|v\|_{\varepsilon}^{2}-\lambda\|v\|_{0}^{2} \geqslant\left(1-C_{K} h-\frac{\lambda}{\eta}\right)\|v\|_{\varepsilon}^{2} .
$$

Zudem erhält man mit Lemma 2.2.2, (1.8) und der Youngschen Ungleichung

$$
\begin{aligned}
e^{\lambda t}\left\langle F_{h}(t), v\right\rangle & \leqslant e^{\lambda t}\|f(t)\|_{0}\|\hat{v}\|_{0} \leqslant e^{\lambda t} \frac{\sqrt{C_{d}}}{\sqrt{\eta}}\|f(t)\|_{0}\|v\|_{\varepsilon} \\
& \leqslant \frac{1}{2}\left(e^{2 \lambda t} \frac{C_{d}}{\delta \eta}\|f(t)\|_{0}^{2}+\delta\|v\|_{\varepsilon}^{2}\right)
\end{aligned}
$$

für jedes $v \in V_{h}=P_{0}^{1}\left(\mathcal{T}_{h}\right)$ und $\delta>0$.

Sei nun $0 \leqslant \lambda<\eta\left(1-C_{K} h\right)$ und $\delta=1-C_{K} h-\frac{\lambda}{\eta}$. Analog zum Beweis des Lemmas 1.1.5 ergibt sich dann mit der Wahl $v=u_{h, \lambda}$ in (3.19) sowie mit (3.20) und (3.21)

$$
\frac{d}{d t}\left\|u_{h, \lambda}(t)\right\|_{0}^{2}+\left(1-C_{K} h-\frac{\lambda}{\eta}\right)\left\|u_{h, \lambda}(t)\right\|_{\varepsilon}^{2} \leqslant \frac{C_{d}}{\eta\left(1-C_{K} h\right)-\lambda} e^{2 \lambda t}\|f(t)\|_{0}^{2} .
$$


Anschließende Integration über $(0, t)$ und Multiplikation mit $e^{-2 \lambda t}$ vervollständigen den Beweis der ersten Abschätzung.

Für die zweite Abschätzung beachte man, daß für die Bilinearform $a_{h}$ wegen Lemma 3.1.1

$$
a_{h}(u, v)=\int_{\Omega} \nabla v \cdot A \nabla u d x+\sum_{B \in \mathcal{B}_{h}}\left(\int_{B} c u \hat{v} d x+\int_{\partial B} b u \hat{v} \cdot d s\right)
$$

gilt. Analog zu Lemma 1.1.5 ergibt sich

$$
\begin{aligned}
\left\|u_{h}^{\prime}(t)\right\|_{0}^{2}+\frac{1}{2} \frac{d}{d t}\left\|A^{\frac{1}{2}} \nabla u_{h}(t)\right\|_{0}^{2} & \\
& \leqslant\|f(t)\|_{0}\left\|\widehat{u_{h}^{\prime}}(t)\right\|_{0}+\left\|\nabla \cdot\left(u_{h}(t) b\right)\right\|_{0}\left\|\widehat{u_{h}^{\prime}}(t)\right\|_{0}+\|c\|_{\infty}\left\|u_{h}(t)\right\|\left\|_{0}\right\| \widehat{u_{h}^{\prime}}(t) \|_{0} .
\end{aligned}
$$

Mit Lemma 2.2.2 ist daher

$$
\begin{aligned}
\left\|u_{h}^{\prime}(t)\right\|_{0}^{2}+\frac{1}{2} \frac{d}{d t}\left\|A^{\frac{1}{2}} \nabla u_{h}(t)\right\|_{0}^{2} & \\
& \leqslant \sqrt{C_{d}}\left(\|f(t)\|_{0}+\left\|\nabla \cdot\left(u_{h}(t) b\right)\right\|_{0}+\|c\|_{\infty}\left\|u_{h}(t)\right\|_{0}\right)\left\|u_{h}^{\prime}(t)\right\|_{0} .
\end{aligned}
$$

Die weitere Argumentation verläuft wie in Lemma 1.1.5.

Für $\lambda=0$ lautet (3.17)

$$
\left\|u_{h}(t)\right\|_{0}^{2}+\left(1-C_{K} h\right) \int_{0}^{t}\left\|u_{h}(s)\right\|_{\varepsilon}^{2} d s \leqslant\left\|u_{0, h}\right\|_{0}^{2}+\frac{C_{d}}{\eta\left(1-C_{K} h\right)} \int_{0}^{t}\|f(s)\|_{0}^{2} d s,
$$

stellt also im Grenzwert $h \rightarrow 0$ bis auf die Konstante $C_{d}$ gerade die Abschätzung (1.12) dar. Nach diesen Vorbereitungen sind wir nun in der Lage, unser erstes a-priori-Resultat zu formulieren unter Ausnutzung der Konsistenzfehlerabschätzung aus Abschnitt 3.1.

Satz 3.3.3 Es sei (TR) vorausgesetzt und $u \in H^{1}(0, T ; V)$ Lösung von (1.2)-(1.4) sowie $u_{h} \in H^{1}\left(0, T ; V_{h}\right)$ mit $V_{h}=P_{0}^{1}\left(\mathcal{T}_{h}\right)$ Lösung der zugehörigen Finite-Volumen-Diskretisierung (3.1)-(3.3) zum Donald-Diagramm $\mathcal{B}_{h}^{D}$ zu $\mathcal{T}_{h}$. Ferner sei Bedingung (1.8) und die Regularitätsforderung aus Lemma 3.3 .1 an das adjungierte Problem (3.15) erfüllt. Dann gilt fast überall in $(0, T)$ die Abschätzung

$$
\begin{aligned}
\left\|e_{h}(t)\right\|_{0}^{2}+\int_{0}^{t}\left\|e_{h}(s)\right\|_{\varepsilon}^{2} d s \leqslant & \left\|e_{h}(0)\right\|_{0}^{2}+\frac{h^{2}}{1-C_{K} h} C_{K}^{2} C_{1}\left\|u_{0, h}\right\|_{0}^{2} \\
& +\left(\frac{1}{\varepsilon}+\frac{C_{K} C_{d}}{\eta\left(1-C_{K} h\right)^{2}}\right) h^{2} C_{1} \int_{0}^{t}\|f(s)\|_{0}^{2} d s \\
& +h^{2} C_{2} \int_{0}^{t}\|u(s)\|_{2}^{2} d s+h^{2} \int_{0}^{t}\left\|u^{\prime}(s)\right\|_{0}^{2}+\left\|u_{h}^{\prime}(s)\right\|_{0}^{2} d s
\end{aligned}
$$

mit $C_{1}:=4 M_{\|\cdot\|_{\varepsilon}}^{2}, C_{2}:=2 C_{R}^{2}\left(1+M_{\|\cdot\|_{\varepsilon}}^{2}\right)$, sofern $h<\frac{1}{C_{K}}$ und $u(t) H^{2}(\Omega)$-regulär ist für fast alle $t \in(0, T)$. 
Beweis. $\quad$ Es sei $u \in H^{1}(0, T ; V)$ Lösung von $(1.2)$ und $u_{h} \in L^{2}\left(0, T ; V_{h}\right)$ Lösung von (2.4). Dann gilt die Fehlergleichung (3.4) insbesondere für $v=R_{h} e_{h}(t)$ für fast jedes $t \in(0, T)$. Mit der Koerzivität und der Stetigkeit von $a$ (siehe (3.11) bzw. (3.13)) sowie

$$
\frac{1}{2} \frac{d}{d t}\left\|e_{h}\right\|_{0}^{2}=\frac{1}{2} \frac{d}{d t}\left(e_{h}, e_{h}\right)=\left\langle e_{h}^{\prime}, e_{h}\right\rangle
$$

(Satz A.3.4) folgt daher

$$
\begin{aligned}
& \frac{1}{2} \frac{d}{d t}\left\|e_{h}(t)\right\|_{0}^{2}+\left\|e_{h}(t)\right\|_{\varepsilon}^{2} \leqslant \frac{1}{2} \frac{d}{d t}\left\|e_{h}(t)\right\|_{0}^{2}+a\left(e_{h}(t), e_{h}(t)\right) \\
&=\quad\left(e_{h}^{\prime}(t), e_{h}(t)\right)+a\left(e_{h}(t), e_{h}(t)\right) \\
&-\left(\left(e_{h}^{\prime}(t), R_{h} e_{h}(t)\right)+a\left(e_{h}(t), R_{h} e_{h}(t)\right)+A_{h}\left(t, R_{h} e_{h}(t)\right)-F_{h}\left(t, R_{h} e_{h}(t)\right)\right. \\
&=\left(e_{h}^{\prime}(t),\left(R_{h}-1\right) u(t)\right)+a\left(e_{h}(t),\left(R_{h}-1\right) u(t)\right)-A_{h}\left(t, R_{h} e_{h}(t)\right)+F_{h}\left(t, R_{h} e_{h}(t)\right) \\
& \leqslant\left\|e_{h}^{\prime}(t)\right\|_{0}\left\|\left(R_{h}-1\right) u(t)\right\|_{0}+M_{\|\cdot\|_{\varepsilon}}\left\|e_{h}(t)\right\|_{\varepsilon}\left\|\left(R_{h}-1\right) u(t)\right\|_{\varepsilon} \\
&+\left|A_{h}\left(t, R_{h} e_{h}(t)\right)\right|+\left|F_{h}\left(t, R_{h} e_{h}(t)\right)\right|
\end{aligned}
$$

mit den durch die Fehlergleichung (3.4) eingeführten Größen $A_{h}(t, v)=a\left(u_{h}(t), v\right)-$ $a_{h}\left(u_{h}(t), v\right)$ und $F_{h}(t, v)=(f(t), v)-\left\langle F_{h}(t), v\right\rangle$.

Wegen der Konsistenzfehlerabschätzungen (3.7) und (3.8) aus Lemma 3.1.3 gilt

$$
\left|A_{h}(t, v)\right|+\left|F_{h}(t, v)\right| \leqslant h\left(C_{K}\left\|u_{h}(t)\right\|_{\varepsilon}+\frac{\|f(t)\|_{0}}{\sqrt{\varepsilon}}\right)\|v\|_{\varepsilon}
$$

für jedes $v \in V$, dabei ist $C_{K}=\frac{\|b\|_{\infty}}{\varepsilon}+\frac{\|\nabla \cdot b+c\|_{\infty}}{\sqrt{\varepsilon \eta}}$. Es ergibt sich

$$
\begin{aligned}
\frac{1}{2} \frac{d}{d t}\left\|e_{h}(t)\right\|_{0}^{2}+\left\|e_{h}(t)\right\|_{\varepsilon}^{2} \leqslant & \left\|e_{h}^{\prime}(t)\right\|_{0}\left\|\left(R_{h}-1\right) u(t)\right\|_{0}+M_{\|\cdot\|_{\varepsilon}}\left\|e_{h}(t)\right\|_{\varepsilon}\left\|\left(R_{h}-1\right) u(t)\right\|_{\varepsilon} \\
& +M_{\|\cdot\|_{\varepsilon}} h\left(C_{K}\left\|u_{h}(t)\right\|_{\varepsilon}+\frac{\|f(t)\|_{0}}{\sqrt{\varepsilon}}\right)\left\|e_{h}(t)\right\|_{\varepsilon},
\end{aligned}
$$

indem man $\left\|R_{h} v\right\|_{\varepsilon} \leqslant M_{\|\cdot\|_{\varepsilon}}\|v\|_{\varepsilon}$ für Funktionen $v \in V$ ausnutzt (siehe Lemma 3.3.1).

Nutzt man zudem die $H^{2}$-Regularitätsbedingung an das adjungierte Problem (3.15) und an $u(t)$ für fast alle $t \in(0, T)$, so liefern die Abschätzungen (3.16)

$$
\begin{aligned}
\frac{1}{2} \frac{d}{d t}\left\|e_{h}(t)\right\|_{0}^{2}+\left\|e_{h}(t)\right\|_{\varepsilon}^{2} \leqslant & C_{R} h^{2}\left\|e_{h}^{\prime}(t)\right\|_{0}\|u(t)\|_{2}+M_{\|\cdot\|_{\varepsilon}} C_{R} h\left\|e_{h}(t)\right\|_{\varepsilon}\|u(t)\|_{2} \\
& +M_{\|\cdot\|_{\varepsilon}} h\left(C_{K}\left\|u_{h}(t)\right\|_{\varepsilon}+\frac{\|f(t)\|_{0}}{\sqrt{\varepsilon}}\right)\left\|e_{h}(t)\right\|_{\varepsilon} .
\end{aligned}
$$

Wir schätzen die drei Terme auf der rechten Seite dieser Ungleichung mit Hilfe der Dreiecksungleichung für $\left\|e_{h}^{\prime}(t)\right\|_{0}$ und der Youngschen Ungleichung einzeln ab:

$$
\begin{aligned}
C_{R} h^{2}\left\|e_{h}^{\prime}(t)\right\|_{0}\|u(t)\|_{2} & \leqslant C_{R}^{2} h^{2}\|u(t)\|_{2}^{2}+\frac{1}{4} h^{2}\left(\left\|u^{\prime}(t)\right\|_{0}+\left\|u_{h}^{\prime}(t)\right\|_{0}\right)^{2} \\
& \leqslant C_{R}^{2} h^{2}\|u(t)\|_{2}^{2}+\frac{1}{2} h^{2}\left(\left\|u^{\prime}(t)\right\|_{0}^{2}+\left\|u_{h}^{\prime}(t)\right\|_{0}^{2}\right),
\end{aligned}
$$




$$
\begin{gathered}
M_{\|\cdot\|_{\varepsilon}} C_{R} h\left\|e_{h}(t)\right\|_{\varepsilon}\|u(t)\|_{2} \leqslant M_{\|\cdot\|_{\varepsilon}}^{2} C_{R}^{2} h^{2}\|u(t)\|_{2}^{2}+\frac{1}{4}\left\|e_{h}(t)\right\|_{\varepsilon}^{2}, \\
M_{\|\cdot\|_{\varepsilon}} h\left(C_{K}\left\|u_{h}(t)\right\|_{\varepsilon}+\frac{\|f(t)\|_{0}}{\sqrt{\varepsilon}}\right)\left\|e_{h}(t)\right\|_{\varepsilon} \\
\leqslant M_{\|\cdot\|_{\varepsilon}}^{2} h^{2}\left(C_{K}\left\|u_{h}(t)\right\|_{\varepsilon}+\frac{\|f(t)\|_{0}}{\sqrt{\varepsilon}}\right)^{2}+\frac{1}{4}\left\|e_{h}(t)\right\|_{\varepsilon}^{2} \\
\leqslant 2 M_{\|\cdot\|_{\varepsilon}}^{2} h^{2}\left(C_{K}^{2}\left\|u_{h}(t)\right\|_{\varepsilon}^{2}+\frac{\|f(t)\|_{0}^{2}}{\varepsilon}\right)+\frac{1}{4}\left\|e_{h}(t)\right\|_{\varepsilon}^{2} .
\end{gathered}
$$

Insgesamt erhält man

$$
\begin{gathered}
\frac{d}{d t}\left\|e_{h}(t)\right\|_{0}^{2}+\left\|e_{h}(t)\right\|_{\varepsilon}^{2} \leqslant h^{2}\left(4 M_{\|\cdot\|_{\varepsilon}}^{2} \frac{\|f(t)\|_{0}^{2}}{\varepsilon}+\left\|u^{\prime}(t)\right\|_{0}^{2}+2 C_{R}^{2}\left(1+M_{\|\cdot\|_{\varepsilon}}^{2}\right)\|u(t)\|_{2}^{2}\right. \\
\left.+\left\|u_{h}^{\prime}(t)\right\|_{0}^{2}+4 C_{K}^{2} M_{\|\cdot\|_{\varepsilon}}^{2}\left\|u_{h}(t)\right\|_{\varepsilon}^{2}\right)
\end{gathered}
$$

und nach Integration über $(0, t)$ und Anwendung von Lemma 3.3 .2 für $\lambda=0$ somit die behauptete Abschätzung.

Bemerkung 3.3.4 Unter weiteren Voraussetzungen kann die folgende a-prioriAbschätzungen Anwendung finden, um den Term $\int_{0}^{t}\|u(s)\|_{2}^{2} d s$ auf der rechten Seite von (3.22) durch die Daten $u_{0}$ und $f$ abzuschätzen.

Zumindest falls $\partial \Omega \in C^{2}$ oder $\Omega$ das Innere eines konvexen Polygons ist, gilt $u(t) \in H^{2}(\Omega)$ und die Abschätzung

$$
\|u(t)\|_{2}^{2} \leqslant C\left(\|L u(t)\|_{0}^{2}+\|u(t)\|_{\varepsilon}^{2}\right)
$$

für fast alle $t \in(0, T)$ (vergl. [42] im ersten, [33] im zweiten Fall). Gegebenenfalls folgt mit $L u=f-u^{\prime}$ sowie den Ungleichungen (1.13) und (1.12)

$$
\begin{aligned}
\int_{0}^{t}\|u(s)\|_{2}^{2} d s \leqslant & C \int_{0}^{t}\|f(s)\|_{0}^{2}+\left\|u^{\prime}(s)\right\|_{0}^{2}+\|u(s)\|_{\varepsilon}^{2} d s \\
\leqslant & C\left[3 \int_{0}^{t}\|f(s)\|_{0}^{2} d s+\left\|A^{\frac{1}{2}} \nabla u_{0}\right\|_{0}^{2}\right. \\
& \left.+\left(4 C_{\varepsilon, \eta}\left(\|\nabla \cdot b\|_{\infty}^{2}+\|b\|_{\infty}^{2}+\|c\|_{\infty}^{2}\right)+1\right) \int_{0}^{t}\|u(s)\|_{\varepsilon}^{2} d s\right] \\
\leqslant & C\left[\left(3+\frac{4}{\eta} C_{\varepsilon, \eta}\left(\|\nabla \cdot b\|_{\infty}^{2}+\|b\|_{\infty}^{2}+\|c\|_{\infty}^{2}\right)+\frac{1}{\eta}\right) \int_{0}^{t}\|f(s)\|_{0}^{2} d s\right. \\
& \left.+\left(4 C_{\varepsilon, \eta}\left(\|\nabla \cdot b\|_{\infty}^{2}+\|b\|_{\infty}^{2}+\|c\|_{\infty}^{2}\right)+1\right)\left\|u_{0}\right\|_{0}^{2}+\left\|A^{\frac{1}{2}} \nabla u_{0}\right\|_{0}^{2}\right]
\end{aligned}
$$

$\operatorname{mit} C_{\varepsilon, \eta}:=\max \left\{\frac{1}{\varepsilon}, \frac{1}{\eta}\right\}$. 
Bezüglich der $L^{2}$-Norm läßt sich - wie bei der Anwendung verallgemeinerter GalerkinVerfahren auf parabolische Probleme zu erwarten - eine Abschätzung herleiten, die zeigt, daß der Einfluß der Anfangswerte exponentiell in der Zeit abklingt (vergl. Bemerkung 1.1.2 zur Glättungseigenschaft parabolischer Probleme). Unter geeigneten Regularitätsanforderungen ist diese sogar von der Ordnung $O\left(h^{2}\right)$, da für das Finite-Volumen-Verfahren ein Konsistenzfehler dieser Güte vorliegt (siehe Abschnitt 3.1). Damit werden die bekannten Fehlerabschätzungen im Fall einer Finite-Element-Diskretisierung mit $P_{1}$-Elementen qualitativ erreicht.

Satz 3.3.5 Es sei (TR) vorausgesetzt und $u \in H^{1}(0, T ; V)$ Lösung von (1.2)-(1.4) sowie $u_{h} \in H^{1}\left(0, T ; V_{h}\right)$ mit $V_{h}=P_{0}^{1}\left(\mathcal{T}_{h}\right)$ Lösung der zugehörigen Finite-Volumen-Diskretisierung (3.1)-(3.3) zum Donald-Diagramm $\mathcal{B}_{h}^{D} z u \mathcal{T}_{h}, h<\frac{1}{2 C_{K}}$. Ferner sei Bedingung (1.8) und die Regularitätsforderung aus Lemma 3.3.1 an das adjungierte Problem (3.15) erfüllt. Dann gilt fast überall in $(0, T)$ die Abschätzung

$$
\begin{aligned}
\left\|u(t)-u_{h}(t)\right\|_{0}^{2} \leqslant & 4 e^{-\eta t}\left\|u_{0}-u_{0, h}\right\|_{0}^{2}+2 h^{2}\left(2 C_{R}^{2} e^{-\eta t}\left|u_{0}\right|_{1}^{2}+\frac{C_{1} e^{-\eta t}}{1-C_{2} h}\left\|u_{0, h}\right\|_{0}^{2}\right. \\
& +C_{R}^{2}|u(t)|_{1}^{2}+\frac{C_{R}^{2}}{\eta} \int_{0}^{t} e^{-\eta(t-s)}\left|u^{\prime}(s)\right|_{1}^{2} d s \\
& \left.+\left(\frac{1}{\varepsilon}+\frac{C_{1} C_{d}}{\left(1-C_{2} h\right) \eta}\right) \int_{0}^{t} e^{-\eta(t-s)}\|f(s)\|_{0}^{2} d s\right),
\end{aligned}
$$

falls $u^{\prime} \in L^{2}\left(0, T ; H^{1}(\Omega)\right)$, und die Abschätzung

$$
\begin{aligned}
\left\|u(t)-u_{h}(t)\right\|_{0}^{2} \leqslant & 4 e^{-\eta t}\left\|u_{0}-u_{0, h}\right\|_{0}^{2}+2 h^{4}\left(2 C_{R}^{2} e^{-\eta t}\left|u_{0}\right|_{2}^{2}+\frac{\tilde{C}_{1} e^{-\eta t}}{1-C_{2} h}\left\|u_{0, h}\right\|_{0}^{2}\right. \\
& +C_{R}^{2}|u(t)|_{2}^{2}+\frac{C_{R}^{2}}{\eta} \int_{0}^{t} e^{-\eta(t-s)}\left|u^{\prime}(s)\right|_{2}^{2} d s \\
& \left.+\left(\frac{C_{P}^{2}}{\varepsilon}+\frac{\tilde{C}_{1} C_{d}}{\left(1-C_{2} h\right) \eta}\right) \int_{0}^{t} e^{-\eta(t-s)}\|f(s)\|_{0}^{2} d s\right),
\end{aligned}
$$

falls sogar $u, u^{\prime} \in L^{2}\left(0, T ; H^{2}(\Omega)\right)$ und die Daten den verschärften Regularitätsbedingungen aus Lemma 3.1.2 genügen. Dabei ist $C_{1}:=2 C_{K}^{2}, \tilde{C}_{1}:=2 \tilde{C}_{K}^{2}$ und $C_{2}:=2 C_{K}$.

Beweis. Wesentlich für den Beweis ist die Zerlegung $e_{h}=u-u_{h}=\rho+\sigma$ mit $\rho:=(1-$ $\left.R_{h}\right) u, \sigma:=R_{h} u-u_{h}$. Für jedes $v \in V_{h}$ gilt dann mit der Fehlergleichung (3.4)

$$
\begin{aligned}
\left(\sigma^{\prime}(t), v\right)+a(\sigma(t), v) & =\left(\left(R_{h} u\right)^{\prime}(t), v\right)+a\left(R_{h} u(t), v\right)-\left(u_{h}^{\prime}(t), v\right)-a\left(u_{h}(t), v\right) \\
& =\left(\left(R_{h} u\right)^{\prime}(t), v\right)+\left(e_{h}^{\prime}(t), v\right)+a\left(e_{h}(t), v\right)-\left(u^{\prime}(t), v\right) \\
& =\left(\left(\left(R_{h}-1\right) u\right)^{\prime}(t), v\right)-A_{h}(t, v)+F_{h}(t, v) \\
& \leqslant\left\|\rho^{\prime}(t)\right\|_{0}\|v\|_{0}+\left|A_{h}(t, v)\right|+\left|F_{h}(t, v)\right| .
\end{aligned}
$$

Insbesondere folgt, da $\sigma(t) \in V_{h}$, mit der Koerzivität (3.11) von $a$ und partieller Integration bezüglich der Zeit (Satz A.3.4)

$$
\frac{1}{2} \frac{d}{d t}\|\sigma(t)\|_{0}^{2}+\|\sigma(t)\|_{\varepsilon}^{2} \leqslant\left\|\rho^{\prime}(t)\right\|_{0}\|\sigma(t)\|_{0}+\left|A_{h}(t, \sigma(t))\right|+\left|F_{h}(t, \sigma(t))\right| .
$$


Im Beweis des Lemmas 3.1.3 wurden die Konsistenzfehlerabschätzungen

$$
\left|a(u, v)-a_{h}(u, v)\right| \leqslant h C_{K}\|u\|_{\varepsilon}\left\|A^{\frac{1}{2}} \nabla v\right\|_{0} \quad \text { und } \quad|f(v-\hat{v})| \leqslant h \frac{\|f\|_{0}}{\sqrt{\varepsilon}}\left\|A^{\frac{1}{2}} \nabla v\right\|_{0}
$$

mit $C_{K}=\frac{\|b\|_{\infty}}{\varepsilon}+\frac{\|\nabla \cdot b+c\|_{\infty}}{\sqrt{\varepsilon \eta}}$ hergeleitet, also ist

$$
\left|A_{h}(t, v)\right|+\left|F_{h}(t, v)\right| \leqslant h\left(C_{K}\left\|u_{h}(t)\right\|_{\varepsilon}+\frac{\|f(t)\|_{0}}{\sqrt{\varepsilon}}\right)\left\|A^{\frac{1}{2}} \nabla v\right\|_{0} .
$$

Mit Youngscher Ungleichung folgt deshalb für jedes $\delta>0$

$$
\begin{aligned}
\frac{1}{2} \frac{d}{d t}\|\sigma(t)\|_{0}^{2}+\|\sigma(t)\|_{\varepsilon}^{2} \leqslant & \left\|\rho^{\prime}(t)\right\|\left\|_{0}\right\| \sigma(t)\left\|_{0}+h\left(C_{K}\left\|u_{h}(t)\right\|_{\varepsilon}+\frac{\|f(t)\|_{0}}{\sqrt{\varepsilon}}\right)\right\| A^{\frac{1}{2}} \nabla \sigma(t) \|_{0} \\
\leqslant & \frac{1}{4 \delta}\left\|\rho^{\prime}(t)\right\|_{0}^{2}+\delta\|\sigma(t)\|_{0}^{2}+\frac{1}{4} h^{2}\left(C_{K}\left\|u_{h}(t)\right\|_{\varepsilon}+\frac{\|f(t)\|_{0}}{\sqrt{\varepsilon}}\right)^{2} \\
& +\left\|A^{\frac{1}{2}} \nabla \sigma(t)\right\|_{0}^{2} \\
\leqslant & \frac{1}{4 \delta}\left\|\rho^{\prime}(t)\right\|_{0}^{2}+\delta\|\sigma(t)\|_{0}^{2}+\frac{1}{2} h^{2}\left(C_{K}^{2}\left\|u_{h}(t)\right\|_{\varepsilon}^{2}+\frac{\|f(t)\|_{0}^{2}}{\varepsilon}\right) \\
& +\left\|A^{\frac{1}{2}} \nabla \sigma(t)\right\|_{0}^{2} .
\end{aligned}
$$

Nach (1.8) ist

$$
\eta\|\sigma(t)\|_{0} \leqslant\|\sqrt{q} \sigma(t)\|^{2}=\|\sigma(t)\|_{\varepsilon}^{2}-\left\|A^{\frac{1}{2}} \nabla \sigma(t)\right\|_{0}^{2}
$$

Dies führt zusammen mit der Wahl $\delta=\frac{\eta}{2}$ in der vorausgegangenen Ungleichung auf

$$
\frac{d}{d t}\|\sigma(t)\|_{0}^{2}+\eta\|\sigma(t)\|_{0}^{2} \leqslant \frac{1}{\eta}\left\|\rho^{\prime}(t)\right\|_{0}^{2}+h^{2}\left(C_{K}^{2}\left\|u_{h}(t)\right\|_{\varepsilon}^{2}+\frac{\|f(t)\|_{0}^{2}}{\varepsilon}\right)
$$

Wegen $\frac{d}{d t} e^{\eta t}\|v(t)\|^{2}=e^{\eta t}\left(\frac{d}{d t}\|v(t)\|^{2}+\eta\|v(t)\|^{2}\right)$ ist damit

$$
\frac{d}{d t}\left(e^{\eta t}\|\sigma(t)\|_{0}^{2}\right) \leqslant e^{\eta t}\left\|\left(1-R_{h}\right) u^{\prime}(t)\right\|_{0}^{2}+h^{2} e^{\eta t}\left(C_{K}^{2}\left\|u_{h}(t)\right\|_{\varepsilon}^{2}+\frac{\|f(t)\|_{0}^{2}}{\varepsilon}\right),
$$

denn $R_{h}$ kommutiert mit dem Zeitableitungsoperator. Integration über $(0, t)$ liefert

$$
\begin{aligned}
\|\sigma(t)\|_{0}^{2} \leqslant & e^{-\eta t}\|\sigma(0)\|_{0}^{2}+\frac{1}{\eta} \int_{0}^{t} e^{-\eta(t-s)}\left\|\left(1-R_{h}\right) u^{\prime}(s)\right\|_{0}^{2} d s \\
& +h^{2} C_{K}^{2} \int_{0}^{t} e^{-\eta(t-s)}\left\|u_{h}(s)\right\|_{\varepsilon}^{2} d s+\frac{h^{2}}{\varepsilon} \int_{0}^{t} e^{-\eta(t-s)}\|f(s)\|_{0}^{2} d s .
\end{aligned}
$$


Mit der Dreiecksungleichung für $\|\rho+\sigma\|_{0}=\left\|u-u_{h}\right\|_{0}$ ist folglich

$$
\begin{aligned}
\left\|u(t)-u_{h}(t)\right\|_{0}^{2} \leqslant 2 & \left(\|\rho(t)\|_{0}^{2}+\|\sigma(t)\|_{0}^{2}\right) \\
\leqslant 2( & \left\|\left(1-R_{h}\right) u(t)\right\|_{0}^{2}+e^{-\eta t}\left\|R_{h} u_{0}-u_{0, h}\right\|_{0}^{2} \\
& +\frac{1}{\eta} \int_{0}^{t} e^{-\eta(t-s)}\left\|\left(1-R_{h}\right) u^{\prime}(t)\right\|_{0}^{2} d s \\
& +h^{2} C_{K}^{2} \int_{0}^{t} e^{-\eta(t-s)}\left\|u_{h}(s)\right\|_{\varepsilon}^{2} d s \\
& \left.+\frac{h^{2}}{\varepsilon} \int_{0}^{t} e^{-\eta(t-s)}\|f(s)\|_{0}^{2} d s\right)
\end{aligned}
$$

fast überall in $(0, T)$.

Nach Voraussetzung ist $\frac{1}{2}<1-C_{K} h$. Es kann daher in Lemma $3.3 .2 \lambda=\frac{\eta}{2}$ gewählt werden, womit man

$$
\int_{0}^{t} e^{-\eta(t-s)}\left\|u_{h}(s)\right\|_{\varepsilon}^{2} d s \leqslant \frac{2 e^{-\eta t}}{1-2 C_{K} h}\left\|u_{0, h}\right\|_{0}^{2}+\frac{2 C_{d}}{\left(1-2 C_{K} h\right) \eta} \int_{0}^{t} e^{-\eta(t-s)}\|f(s)\|_{0}^{2} d s
$$

erhält. Nutzt man zudem

$$
\left\|R_{h} u_{0}-u_{0, h}\right\|_{0}^{2} \leqslant 2\left(\left\|u_{0}-u_{0, h}\right\|_{0}^{2}+\left\|\left(R_{h}-1\right) u_{0}\right\|_{0}^{2}\right)
$$

und aufgrund der Regularitätsforderung an das adjungierte Problem (3.15) die Abschätzungen (3.16) angewendet auf $u, u^{\prime} \in L^{2}\left(0, T ; H^{1}(\Omega)\right)$, ergibt sich somit (3.23).

Unter den verschärften Bedingungen an die Problemdaten,

$$
b \in H^{1, \infty}(\Omega)^{d}, \quad \nabla \cdot b \in H^{1, \infty}(\Omega), \quad c \in H^{1, \infty}(\Omega), \quad f \in L^{2}\left(0, T ; H^{1}(\Omega)\right),
$$

folgt mit (3.9) und (3.10)

$$
\left|A_{h}(t, v)\right|+\left|F_{h}(t, v)\right| \leqslant h^{2}\left(\tilde{C}_{K}\left\|A^{\frac{1}{2}} \nabla u_{h}(t)\right\|_{0}+\frac{C_{P}}{\sqrt{\varepsilon}}\|f(t)\|_{1}\right)\left\|A^{\frac{1}{2}} \nabla v\right\|_{0},
$$

mithin ergibt sich analog zum ersten Teil des Beweises

$$
\begin{aligned}
\|\sigma(t)\|_{0}^{2} \leqslant & e^{-\eta t}\|\sigma(0)\|_{0}^{2}+\frac{1}{\eta} \int_{0}^{t} e^{-\eta(t-s)}\left\|\left(1-R_{h}\right) u^{\prime}(s)\right\|_{0}^{2} d s \\
& +h^{4} \tilde{C}_{K}^{2} \int_{0}^{t} e^{-\eta(t-s)}\left\|A^{\frac{1}{2}} \nabla u_{h}(s)\right\|_{0}^{2} d s+h^{4} \frac{C_{P}^{2}}{\varepsilon} \int_{0}^{t} e^{-\eta(t-s)}\|f(s)\|_{1}^{2} d s .
\end{aligned}
$$

Nimmt man zusätzlich die Lösung $u$ und deren Ableitung $u^{\prime}$ in $L^{2}\left(0, T ; H^{2}(\Omega)\right)$ an, so erhält die Fehlerabschätzung infolge der Abschätzung (3.16) für den Ritz-Operator mit $k=1$ die Gestalt (3.24).

Die Terme $e^{-\eta t}|| u_{0}-\left.u_{0, h}\right|_{0} ^{2}$ und $e^{-\eta t}\left|u_{0}\right|_{1}^{2}$ zeigen die erwähnte Glättungseigenschaft in Bezug auf die Anfangswerte. Man beachte, daß der mit der Zeit exponentiell dämpfende Faktor $e^{-\eta t}$, der hier und an weiteren Stellen in den Fehlerabschätzungen (3.23) und (3.24) auftritt, unabhängig von der Kleinheit der Diffusion $\varepsilon$ ist. 


\subsection{A-priori-Fehlerabschätzungen zum volldiskreten Problem}

In diesem Abschnitt gelangen wir zu einer vollständigen Diskretisierung des kontinuierlichen Problems (1.2), indem wir an dessen Finite-Volumen-Diskretisierung eine Zeitdiskretisierung durch das $\theta$-Schema anschließen. Das so entstehende Verfahren untersuchen wir auf Stabilität und Konvergenz.

$\mathrm{Zu}$ diesem Zweck betrachten wir Probleme mit einem Diffusionsterm der einfachen Form $\nabla v \cdot A \nabla u=\varepsilon \nabla v \cdot \nabla u, \varepsilon>0$, also mit Bilinearform

$$
a(u, v)=\int_{\Omega} \varepsilon \nabla v \cdot \nabla u+\left(\frac{1}{2} \nabla \cdot b+c\right) u v d x+\frac{1}{2} \int_{\Omega} v b \cdot \nabla u-u b \cdot \nabla v d x .
$$

Wie zuvor gelte

$$
\eta:=\underset{\Omega}{\operatorname{essinf}} q>0 \text { mit } q:=\frac{1}{2} \nabla \cdot b+c .
$$

Bereits im Abschnitt 1.2 wurde darauf hingewiesen, das die Semidiskretisierung von Evolutionsproblemen (1.1) in Systeme der Form

$$
M \mathbf{x}^{\prime}(t)+A \mathbf{x}(t)=\mathbf{F}(t)
$$

mit entsprechenden Anfangsbedingungen mündet. Hieran kann ein geeignetes Verfahren zur Lösung von gewöhnlichen Differentialgleichungssytemen anschließen, um in der Zeit zu diskretisieren, etwa ein Runge-Kutta- oder diskontinuierliches Galerkin-Verfahren.

Wir wählen das $\theta$-Schema, da hier durch eine einfache Stabilitäts- und Konvergenzanalyse der Gesamtdiskretisierungsfehler direkt angegeben werden kann. Im Allgemeinen kann eine Abschätzung des Gesamtdiskretisierungsfehlers aus dem Fehler zwischen semidiskreter und volldiskreter Lösung sowie den im vorangegangenen Abschnitt bewiesenen Abschätzungen zum semidiskreten Problem zusammengesetzt werden.

Das $\theta$-Schema, $\theta \in[0,1]$, zur Approximation eines Anfangswertproblems

$$
\begin{aligned}
y^{\prime}(t) & =g(t, y(t)), \quad t \in(0, T) \\
y(0) & =y_{0}
\end{aligned}
$$

und mit der Zeitschrittweite $\Delta t$ lautet

$$
\begin{aligned}
t_{0} & =0, \quad t_{n+1}=t_{n}+\Delta t \\
y_{n+1} & =y_{n}+\Delta t\left(\theta g\left(t_{n+1}, y_{n+1}\right)+(1-\theta) g\left(t_{n}, y_{n}\right)\right)
\end{aligned}
$$

Dies ist ein lineares Einschrittverfahren, das bekanntlich für $\theta=0$ das explizite EulerVerfahren, für $\theta=\frac{1}{2}$ das Crank-Nicolson-Verfahren und für $\theta=1$ das implizite EulerVerfahren darstellt.

Es sei $\theta \in[0,1], N \in \mathbb{N}$ sowie $t_{n}:=n \Delta t$ für $n=0,1, \ldots, N$ mit $\Delta t \in\left(0, \frac{T}{N}\right]$. Dann ergibt das $\theta$-Schema angewendet auf (3.1) das volldiskrete Problem: 
$\mathrm{Zu} n=0,1, \ldots, N-1$ finde $u_{h}^{n+1} \in V_{h}$ mit

$$
\left\{\begin{aligned}
\frac{1}{\Delta t}\left(u_{h}^{n+1}-u_{h}^{n}, v\right)+a_{h}\left(\theta u_{h}^{n+1}+(1-\theta) u_{h}^{n}, v\right) & =\left\langle\theta F_{h}^{n+1}+(1-\theta) F_{h}^{n}, v\right\rangle \\
u_{h}^{0} & =u_{0, h}
\end{aligned}\right.
$$

für alle $v \in V_{h}=P_{0}^{1}\left(\mathcal{T}_{h}\right)$. Dabei sei (TR) vorausgesetzt, $a_{h}$ die Bilinearform (3.2) zum DonaldDiagramm $\mathcal{B}_{h}^{D}$ zu $\mathcal{T}_{h}$ und $\left\langle F_{h}^{n}, v\right\rangle:=\left\langle F_{h}\left(t_{n}\right), v\right\rangle$ gemäß (3.3).

Dies entspricht in jedem Zeitschritt $n \rightarrow n+1$ der Lösung des elliptischen Problems

$$
a_{\theta}\left(u_{h}^{n+1}, v\right)=\left\langle G^{n}, v\right\rangle
$$

für alle $v \in V_{h}$, wobei

$$
\begin{aligned}
a_{\theta}(u, v) & :=\theta a_{h}(u, v)+\frac{1}{\Delta t}(u, v), \\
\left\langle G^{n}, v\right\rangle & :=\left\langle\theta F_{h}^{n+1}+(1-\theta) F_{h}^{n}, v\right\rangle+\frac{1}{\Delta t}\left(u_{h}^{n}, v\right)-(1-\theta) a_{h}\left(u_{h}^{n}, v\right) .
\end{aligned}
$$

Die Existenz einer eindeutig bestimmten Lösung folgt daher mit dem Lax-Milgram-Satz aus der Koerzivität für hinreichend kleine $h$ sowie der Stetigkeit von $a_{h}$ (siehe Abschnitt 3.1).

Für $\theta \in\left[\frac{1}{2}, 1\right]$ ist das Verfahren (3.26), wie sich zeigen wird, unbedingt stabil. Die Stabilität und Konvergenz im Fall $\theta \in\left[0, \frac{1}{2}\right.$ ) (insbesondere also für ein explizites Euler-Verfahren) sichern wir durch die Annahmen

$(\theta 1)\left(\mathcal{T}_{h}\right)_{h>0}$ sei eine quasi-uniforme Familie von Triangulierungen, folglich gilt mit einer Konstante $C_{I}>0$ die inverse Ungleichung

$$
|v|_{1} \leqslant \frac{C_{I}}{h}\|v\|_{0} \quad \text { für jedes } v \in V_{h},
$$

( $\theta 2)$ für $\Delta t$ gelte die Zeitschrittweitenbeschränkung

$$
\begin{aligned}
\Delta t & \leqslant \frac{h^{2}}{3(1-2 \theta) C_{I}^{2}} \min \left\{\frac{1}{3 \varepsilon}, \frac{\eta}{S_{h}^{2}}\right\} . \\
\text { mit } S_{h} & :=\|b\|_{\infty}+h\left(\frac{\|q\|_{\infty}}{C_{I}}+\|\nabla \cdot b+c\|_{\infty}\right) .
\end{aligned}
$$

Wir zeigen zunächst die Stabilität des Verfahrens. Abkürzend schreiben wir $v^{n+\theta}:=\theta v^{n+1}+$ $(1-\theta) v^{n}$.

Satz 3.4.1 Es gelte (TR) mit $h \leqslant h_{0}:=\frac{\min \{\varepsilon, \eta\}}{2\|b\|_{\infty}+\|\nabla \cdot b+c\|_{\infty}}$. Zudem sei $\theta \in[0,1]$, und im Fall $\theta<\frac{1}{2}$ seien $(\theta 1)$ und $(\theta 2)$ vorausgesetzt. Dann gilt für eine Lösung $\left(u_{h}^{n}\right)_{n \in \underline{N}}, u_{h}^{n} \in V_{h}$, von (3.26)

$$
\left\|u_{h}^{n}\right\|_{0}^{2} \leqslant\left\|u_{0, h}\right\|_{0}^{2}+C\left(1+h_{0}^{2}\right) \frac{\Delta t}{\varepsilon} \sum_{j \in n}\left\|\theta f^{j+1}+(1-\theta) f^{j}\right\|_{0}^{2}, \quad n=1, \ldots, N,
$$

mit einer Konstante $C>0$, die weder von $h$, von $\Delta t$ noch von den Daten des Problems (3.26) abhängt. 
Beweis. $\quad$ Die Wahl $v=u_{h}^{j+\theta}$ in $(3.26)_{1}$ ergibt

$$
\begin{array}{r}
\frac{1}{2}\left\|u_{h}^{j+1}\right\|_{0}^{2}-\frac{1}{2}\left\|u_{h}^{j}\right\|_{0}^{2}+\left(\theta-\frac{1}{2}\right) \| u_{h}^{j+1}- \\
-u_{h}^{j} \|_{0}^{2}+\Delta t\left(a\left(u_{h}^{j+\theta}, u_{h}^{j+\theta}\right)-A_{h}\left(u_{h}^{j+\theta}, u_{h}^{j+\theta}\right)\right) \\
=\Delta t\left(\left(f^{j+\theta}, u_{h}^{j+\theta}\right)-\left(f^{j+\theta}, u_{h}^{j+\theta}-\hat{u}_{h}^{j+\theta}\right)\right)
\end{array}
$$

für jedes $m \in N$ mit $A_{h}=a-a_{h}$ auf $V_{h} \times V_{h}$. Mit der Poincaré-Friedrich-Ungleichung (A.2.6), dem Lemma 2.2.1 und Youngscher Ungleichung folgt

$$
\left(f^{j+\theta}, u_{h}^{j+\theta}\right) \leqslant \sqrt{C_{P F}}\left\|f^{j+\theta}\right\|_{0}\left|u_{h}^{j+\theta}\right|_{1} \leqslant 2 \frac{C_{P F}}{\varepsilon}\left\|f^{j+\theta}\right\|_{0}^{2}+\frac{\varepsilon}{8}\left|u_{h}^{j+\theta}\right|_{1}^{2}
$$

sowie

$$
\left|\left(f^{j+\theta}, u_{h}^{j+\theta}-\hat{u}_{h}^{j+\theta}\right)\right| \leqslant h\left\|f^{j+\theta}\right\|_{0}\left|u_{h}^{j+\theta}\right|_{1} \leqslant 2 \frac{h^{2}}{\varepsilon}\left\|f^{j+\theta}\right\|_{0}^{2}+\frac{\varepsilon}{8}\left|u_{h}^{j+\theta}\right|_{1}^{2} .
$$

Andererseits ist wegen (3.25) und (3.5)

$$
\begin{aligned}
a\left(u_{h}^{j+\theta}, u_{h}^{j+\theta}\right)-A_{h}\left(u_{h}^{j+\theta}, u_{h}^{j+\theta}\right) \geqslant & \left.\left(\varepsilon-h\left(\|b\|_{\infty}+\frac{1}{2}\|\nabla \cdot b+c\|_{\infty}\right)\right)\right)\left|u_{h}^{j+\theta}\right|_{1}^{2} \\
& +\left(\eta-h \frac{1}{2}\|\nabla \cdot b+c\|_{\infty}\right)\left\|u_{h}^{j+\theta}\right\|_{0}^{2} \\
\geqslant & \frac{1}{2}\left(\varepsilon\left|u_{h}^{j+\theta}\right|_{1}^{2}+\eta\left\|u_{h}^{j+\theta}\right\|_{0}^{2}\right)
\end{aligned}
$$

für $h \leqslant h_{0}$. Mit diesen Abschätzungen auf der linken und rechten Seite von (3.29) gelangt man zu

$$
\left\|u_{h}^{j+1}\right\|_{0}^{2}-\left\|u_{h}^{j}\right\|_{0}^{2}+(2 \theta-1)\left\|u_{h}^{j+1}-u_{h}^{j}\right\|_{0}^{2}+\Delta t\left(\frac{\varepsilon}{2}\left|u_{h}^{j+\theta}\right|_{1}^{2}+\eta\left\|u_{h}^{j+\theta}\right\|_{0}^{2}\right) \leqslant 4 \Delta t \frac{C_{P F}+h^{2}}{\varepsilon}\left\|f^{j+\theta}\right\|_{0}^{2} .
$$

Wir unterscheiden nun nach Werten für $\theta$. Im Fall $\theta \in\left[\frac{1}{2}, 1\right]$ ist die linke Seite der vorstehenden Ungleichung größer als $\left\|u_{h}^{j+1}\right\|_{0}^{2}-\left\|u_{h}^{j}\right\|_{0}^{2}$. Im Fall $\theta \in\left[0, \frac{1}{2}\right)$ schätzen wir $\left\|u_{h}^{j+1}-u_{h}^{j}\right\|_{0}^{2}$ ab. Nach $(3.26)_{1}$ gilt nämlich

$$
\frac{1}{\Delta t}\left(u_{h}^{j+1}-u_{h}^{j}, v\right)+a\left(u_{h}^{j+\theta}, v\right) \leqslant\left(f^{j+\theta}, v\right)+\left|A_{h}\left(u_{h}^{j+\theta}, v\right)\right|+\left|\left(f^{j+\theta}, v-\hat{v}\right)\right|
$$

für alle $v \in V_{h}$. Eine Betrachtung der einzelnen Summanden von $a$ und (3.27) liefern

$$
\begin{aligned}
-a\left(u_{h}^{j+\theta}, v\right) \leqslant & \varepsilon\left|u_{h}^{j+\theta}\right|_{1}|v|_{1}+\|q\|_{\infty}\left\|u_{h}^{j+\theta}\right\|\left\|_{0}\right\| v \|_{0} \\
& +\frac{1}{2}\|b\|_{\infty}\left(\left|u_{h}^{j+\theta}\right|_{1}\|v\|_{0}+\left\|u_{h}^{j+\theta}\right\|_{0}|v|_{1}\right) \\
\leqslant & \left(\varepsilon \frac{C_{I}}{h}\left|u_{h}^{j+\theta}\right|_{1}+\left(\|q\|_{\infty}+\frac{C_{I}}{h}\|b\|_{\infty}\right)\left\|u_{h}^{j+\theta}\right\|_{0}\right)\|v\|_{0} .
\end{aligned}
$$


Mit (3.5) und Lemma 2.2.1 sowie wiederum (3.27) ist

$$
\left|A_{h}\left(u_{h}^{j+\theta}, v\right)\right|+\left|\left(f^{j+\theta}, v-\hat{v}\right)\right| \leqslant C_{I}\left(\|b\|_{\infty}\left|u_{h}^{j+\theta}\right|_{1}+\|\nabla \cdot b+c\|_{\infty}\left\|u_{h}^{j+\theta}\right\|_{0}+\left\|f^{j+\theta}\right\|_{0}\right)\|v\|_{0} .
$$

Wählt man nun $v=u_{h}^{j+1}-u_{h}^{j}$, so gelangt man schließlich zu

$$
\begin{aligned}
\left\|u_{h}^{j+1}-u_{h}^{j}\right\|_{0}^{2} \leqslant & 3(\Delta t)^{2} \frac{C_{I}^{2}}{h^{2}}\left[3 \varepsilon^{2}\left|u_{h}^{j+\theta}\right|_{1}^{2}+\left(\|b\|_{\infty}+h\left(\|q\|_{\infty} C_{I}^{-1}+\|\nabla \cdot b+c\|_{\infty}\right)\right)^{2}\left\|u_{h}^{j+\theta}\right\|_{0}^{2}\right. \\
& \left.+h^{2}\left(1+C_{I}^{-1}\right)^{2}\left\|f^{j+\theta}\right\|_{0}^{2}\right]
\end{aligned}
$$

wobei nach Voraussetzung $h \leqslant \frac{\varepsilon}{2\|b\|_{\infty}}$ ausgenutzt wurde. Da in diesem Fall die Zeitschrittweitenbeschränkung $(\theta 2)$ vorausgesetzt wurde, gilt zusammenfassend in beiden Fällen

$$
\left\|u_{h}^{j+1}\right\|_{0}^{2}-\left\|u_{h}^{j}\right\|_{0}^{2} \leqslant \frac{\Delta t}{\varepsilon}\left(C_{1}+h^{2} C_{2}\right)\left\|f^{j+\theta}\right\|_{0}^{2}
$$

für jedes $j \in N$ mit $C_{1}:=4 C_{P F}$ und $C_{2}:=4$ bzw. $C_{2}:=4+\left(1+C_{I}^{-1}\right)^{2}$. Die Behauptung folgt somit nach Summation über $j=0, \ldots, n-1$ und mit $C=\max \left\{C_{1}, C_{2}\right\}$.

Wie dem vorausgegangenen Beweis zu entnehmen ist, geht der Faktor $1+h_{0}^{2}$ in der Stabilitätsabschätzung von Satz 3.4.1 auf die Verwendung der Konsistenzfehlerabschätzung für den Term $(f, v-\hat{v})$ zurück.

Bemerkung 3.4.2 Im Fall $\theta \geqslant \frac{1}{2}$ kann man eine verschärfte Stabilitätsabschätzung gewinnen, bei der die Terme mit Anfangsbedingung $u_{0}$ und Quellfunktion $f$ mit einem dämpfenden Faktor gewichtet sind und die insofern als diskrete Entsprechung der Abschätzung (3.17) angesehen werden kann.

Es gilt nämlich

$$
C_{\theta}:=\frac{1+(1-\theta)(2 \theta-1) \lambda}{1+\theta(2 \theta-1) \lambda} \leqslant 1
$$

und

$$
\begin{array}{r}
\left\|u_{h}^{N}\right\|_{0}^{2}+\sum_{j \in N} \frac{C_{\theta}^{N-j}}{1+\theta(2 \theta-1) \lambda}\left((2 \theta-1)\left\|u_{h}^{j+1}-u_{h}^{j}\right\|_{0}^{2}+\Delta t\left(\frac{\varepsilon}{4}\left|u_{h}^{j+\theta}\right|_{1}^{2}+\frac{\eta}{2}\left\|u_{h}^{j+\theta}\right\|_{0}^{2}\right)\right) \\
\leqslant C_{\theta}^{N}\left\|u_{0, h}\right\|_{0}^{2}+\sum_{j \in N} \frac{C_{\theta}^{N-j} \Delta t}{2 \lambda(1+\theta(2 \theta-1) \lambda)}\left\|f^{j+\theta}\right\|_{0}^{2},
\end{array}
$$

sofern $\theta \leqslant \frac{1}{2}$, wobei $\lambda:=\frac{\varepsilon}{8\left(C_{P F}+h_{0}^{2}\right)}$. Für das Crank-Nicolson-Verfahren, d. h. für $\theta=\frac{1}{2}$, ist jedoch $C_{\theta}=1$ und damit die Dämpfung aufgehoben.

Im Rest des Abschnitts leiten wir eine Konvergenzaussage für das betrachtete Verfahren ab. Es sei $t \in(0, T)$ und $\Delta t=\Delta t(N)=\frac{t}{N}$ zu jedem $N \in \mathbb{N}$, also $t=t_{N}$. Der nächste Satz zeigt 
dann die Konvergenz

$$
u_{h}^{N} \stackrel{N \rightarrow \infty}{\longrightarrow} u(t)
$$

bezüglich der $L^{2}(\Omega)$-Norm, wobei $u_{h}^{N}$ Lösung von (3.26) für $n=N-1$ und $u$ Lösung des kontinuierlichen Problems (1.2) - (1.4) ist.

Satz 3.4.3 Es seien die Voraussetzungen von Satz 3.4 .1 und die Regularitätsforderung aus Lemma 3.3 .1 an das adjungierte Problem (3.15) erfüllt. Ferner sei u eine Lösung von (1.2) - (1.4) und $\left(u_{h}^{n}\right)_{n \in \underline{N}}, u_{h}^{n} \in V_{h}$, eine Lösung von (3.26). Dann gilt für jedes $n \in \underline{N}$ die Abschätzung

$$
\begin{aligned}
\left\|u\left(t_{n}\right)-u_{h}^{n}\right\|_{0}^{2} \leqslant & h^{2} C\left(\left|u_{0}\right|_{1}^{2}+\left|u\left(t_{n}\right)\right|_{1}^{2}+\frac{\Delta t}{\varepsilon} \sum_{j \in n}\left\|f^{j+\theta}\right\|_{0}^{2}\right) \\
& +C \frac{T}{\varepsilon}\left(1+h_{0}^{2}\right)\left(h^{2} \sup _{[0, T]}\left|u^{\prime}(t)\right|_{1}^{2}+(\Delta t)^{2} \sup _{[0, T]}\left\|u^{\prime \prime}(t)\right\|_{0}^{2}\right),
\end{aligned}
$$

falls $u^{\prime} \in L^{\infty}\left(0, T ; H^{1}(\Omega)\right), u^{\prime \prime} \in L^{\infty}\left(0, T ; L^{2}(\Omega)\right)$, und die Abschätzung

$$
\begin{aligned}
\left\|u\left(t_{n}\right)-u_{h}^{n}\right\|_{0}^{2} \leqslant & h^{4} C\left(\left|u_{0}\right|_{2}^{2}+\left|u\left(t_{n}\right)\right|_{2}^{2}\right)+\frac{\Delta t}{\varepsilon} h^{2} C \sum_{j \in n}\left\|f^{j+\theta}\right\|_{0}^{2} \\
& +C \frac{T}{\varepsilon}\left(1+h_{0}^{2}\right)\left(h^{4} \sup _{[0, T]}\left|u^{\prime}(t)\right|_{2}^{2}+(\Delta t)^{2} \sup _{[0, T]}\left\|u^{\prime \prime}(t)\right\|_{0}^{2}\right),
\end{aligned}
$$

falls darüber hinaus sogar $u, u^{\prime} \in L^{\infty}\left(0, T ; H^{2}(\Omega)\right)$. Dabei ist $C>0$ eine Konstante, die weder von $h$, von $\Delta t$ noch von den Daten des Problems (3.26) abhängt.

Beweis. $\quad$ Da die Lösung $u \in H^{1}(0, T, V)$ von $(1.2)$ in $C(0, T ; V)$ liegt, ist die Bezeichnung $u^{j}:=u\left(t_{j}\right)$ sinnvoll. Wiederum zerlegen wir die Differenz der Lösungen, hier in $u^{j}-u_{h}^{j}=\rho^{j}+$ $\sigma^{j}$ mit $\rho^{j}:=\left(1-R_{h}\right) u^{j}, \sigma^{j}:=R_{h} u^{j}-u_{h}^{j}$, um zunächst eine Abschätzung für $\sigma^{j}$ herzuleiten.

Für jedes $v \in V$ gilt nach Definition von $R_{h}$ sowie mit $(3.26)_{1}$ und $(1.2)_{1}$

$$
\begin{aligned}
\frac{1}{\Delta t}\left(\sigma^{j+1}-\sigma^{j}, v\right)+a\left(\sigma^{j+\theta}, v\right)= & \frac{1}{\Delta t}\left(R_{h} u^{j+1}-R_{h} u^{j}, v\right)+a\left(\theta R_{h} u^{j+1}+(1-\theta) R_{h} u^{j}, v\right) \\
& -\frac{1}{\Delta t}\left(u_{h}^{j+1}-u_{h}^{j}, v\right)-a\left(u_{h}^{j+\theta}, v\right)+A_{h}\left(u_{h}^{j+\theta}, v\right) \\
= & \frac{1}{\Delta t}\left(R_{h} u^{j+1}-R_{h} u^{j}, v\right)+a\left(u^{j+\theta}, v\right)-\left(f^{j+\theta}, v\right) \\
& +A_{h}\left(u_{h}^{j+\theta}, v\right)+\left(f^{j+\theta}, v-\hat{v}\right) \\
= & \left(w^{j}, v\right)+A_{h}\left(u_{h}^{j+\theta}, v\right)+\left(f^{j+\theta}, v-\hat{v}\right),
\end{aligned}
$$

wobei $A_{h}:=a-a_{h}$ auf $V_{h} \times V_{h}$ und

$$
\begin{aligned}
w^{j} & :=\frac{1}{\Delta t}\left(R_{h} u^{j+1}-R_{h} u^{j}\right)-\theta\left(u^{\prime}\right)^{j+1}-(1-\theta)\left(u^{\prime}\right)^{j} \\
& =\frac{1}{\Delta t}\left(\left(R_{h}-1\right) u^{j+1}-\left(R_{h}-1\right) u^{j}\right)+\frac{u^{j+1}-u^{j}}{\Delta t}-\theta\left(u^{\prime}\right)^{j+1}-(1-\theta)\left(u^{\prime}\right)^{j} .
\end{aligned}
$$


Mit der Wahl $v=\sigma^{j+\theta}$ folgt dann wie im vorausgegangenen Beweis zur Stabilität

$$
\begin{aligned}
\left\|\sigma^{j+1}\right\|_{0}^{2}-\left\|\sigma^{j}\right\|_{0}^{2}+(2 \theta-1)\left\|\sigma^{j+1}-\sigma^{j}\right\|_{0}^{2}+ & \Delta t\left(\varepsilon\left|\sigma^{j+\theta}\right|_{1}+\eta\left\|\sigma^{j+\theta}\right\|_{0}\right) \\
& \leqslant 2 \Delta t\left(\left(w^{j}, \sigma^{j+\theta}\right)+h\left\|f^{j+\theta}\right\|_{0}\left|\sigma^{j+\theta}\right|_{1}\right)
\end{aligned}
$$

aufgrund der Voraussetzung an $h$. Durch Taylor-Darstellungen (siehe (A.1)) der Summanden von $w^{j}$ und wegen der Eigenschaften des Ritz-Operators $R_{h}$ (siehe Lemma 3.3.1) erhält man für jedes $v \in V_{h}$

$$
\begin{aligned}
\left(\left(R_{h}-1\right) u^{j+1}-\left(R_{h}-1\right) u^{j}, v\right) & =\left(\int_{t_{j}}^{t_{j+1}}\left(R_{h}-1\right) u^{\prime}(t) d t, v\right) \\
& \leqslant \int_{t_{j}}^{t_{j+1}}\left\|\left(R_{h}-1\right) u^{\prime}(t)\right\|_{0} d t\|v\|_{0} \\
& \leqslant \Delta t \sup _{\left[t_{j}, t_{j+1}\right]}\left\|\left(R_{h}-1\right) u^{\prime}(t)\right\|_{0}\|v\|_{0}
\end{aligned}
$$

sowie

$$
\begin{aligned}
\left(u^{j+1}-u^{j}-\Delta t\left(u^{\prime}\right)^{j+\theta}, v\right) & =\left(\int_{t_{j}}^{t_{j+1}}\left(\theta t_{j}+(1-\theta) t_{j+1}-t\right) u^{\prime \prime}(t) d t, v\right) \\
& \leqslant \Delta t \int_{t_{j}}^{t_{j+1}}\left\|u^{\prime \prime}(t)\right\|_{0} d t\|v\|_{0} \\
& \leqslant(\Delta t)^{2} \sup _{\left[t_{j}, t_{j+1}\right]}\left\|u^{\prime \prime}(t)\right\|_{0}\|v\|_{0} .
\end{aligned}
$$

Zieht man (3.35) und (3.36) die Poincaré-Friedrich-Ungleichung (A.2.6) sowie die Youngsche Ungleichung für $\varepsilon$ heran, so gilt infolgedessen

$$
\begin{aligned}
2 \Delta t\left(\left(w^{j}, \sigma^{j+\theta}\right)+h\left\|f^{j+\theta}\right\|_{0}\left|\sigma^{j+\theta}\right|_{1}\right) & \\
\leqslant & 2 \frac{\Delta t}{\varepsilon}\left[h\left\|f^{j+\theta}\right\|_{0}+C_{P F}\left(\sup _{\left[t_{j}, t_{j+1}\right]}\left\|\left(R_{h}-1\right) u^{\prime}(t)\right\|_{0}+\Delta t \sup _{\left[t_{j}, t_{j+1}\right]}\left\|u^{\prime \prime}(t)\right\|_{0}\right)\right]^{2} \\
& +\frac{\Delta t}{2} \varepsilon\left|\sigma^{j+\theta}\right|_{1}^{2} .
\end{aligned}
$$

Zusammen mit (3.34) und der Abkürzung $S_{j}:=\sup _{\left[t_{j}, t_{j+1}\right]}\left\|\left(R_{h}-1\right) u^{\prime}(t)\right\|_{0}+$ $\Delta t \sup _{\left[t_{j}, t_{j+1}\right]}\left\|u^{\prime \prime}(t)\right\|_{0}$ ergibt sich daher

$$
\begin{aligned}
\left\|\sigma^{j+1}\right\|_{0}^{2}-\left\|\sigma^{j}\right\|_{0}^{2}+(2 \theta-1)\left\|\sigma^{j+1}-\sigma^{j}\right\|_{0}^{2}+\Delta t\left(\frac{\varepsilon}{2}\left|\sigma^{j+\theta}\right|_{1}\right. & \left.+\eta\left\|\sigma^{j+\theta}\right\|_{0}\right) \\
& \leqslant 4 \frac{\Delta t}{\varepsilon}\left[h\left\|f^{j+\theta}\right\|_{0}+C_{P F} S_{j}\right]^{2} .
\end{aligned}
$$


Für den Fall $\theta \in\left[0, \frac{1}{2}\right)$ schätzen wir $\left\|\sigma^{j+1}-\sigma^{j}\right\|_{0}^{2}$ ab. Analog zum Beweis zur Stabilität gewinnt man aus (3.33) sowie unter Berücksichtigung von (3.35) und (3.36)

$$
\begin{aligned}
\left\|\sigma^{j+1}-\sigma^{j}\right\|_{0}^{2} \leqslant & 3(\Delta t)^{2} \frac{C_{I}^{2}}{h^{2}}\left[3 \varepsilon^{2}\left|\sigma^{j+\theta}\right|_{1}^{2}+\left(\|b\|_{\infty}+h\left(\|q\|_{\infty} C_{I}^{-1}+\|\nabla \cdot b+c\|_{\infty}\right)\right)^{2}\left\|\sigma^{j+\theta}\right\|_{0}^{2}\right. \\
& \left.+\left(h\left\|f^{j+\theta}\right\|_{0}+\frac{h}{C_{I}} S_{j}\right)^{2}\right]
\end{aligned}
$$

mit der Wahl $v=\sigma^{j+1}-\sigma^{j}$. Da laut Zeitschrittweitenbeschränkung $(\theta 2) \Delta t \leqslant \frac{h^{2}}{3(1-2 \theta) C_{I}^{2}} \frac{1}{\varepsilon}$ gilt, folgt somit

$$
\left\|\sigma^{j+1}\right\|_{0}^{2}-\left\|\sigma^{j}\right\|_{0}^{2} \leqslant 4 \frac{\Delta t}{\varepsilon}\left[h\left\|f^{j+\theta}\right\|_{0}+C_{P F} S_{j}\right]^{2}+\frac{\Delta t}{\varepsilon}\left[h\left\|f^{j+\theta}\right\|_{0}+\frac{h}{C_{I}} S_{j}\right]^{2}
$$

Durch Summation über $j=0, \ldots, n-1$ erhählt man wegen $\Delta t \leqslant \frac{T}{n}$ zusammenfassend für beide Fälle

$$
\begin{aligned}
\left\|\sigma^{n}\right\|_{0}^{2} \leqslant & \left\|\sigma^{0}\right\|_{0}^{2}+C \frac{\Delta t}{\varepsilon} h^{2} \sum_{j \in n}\left\|f^{j+\theta}\right\|_{0}^{2} \\
& +C \frac{\Delta t}{\varepsilon}\left(1+h^{2}\right)\left(\sum_{j \in n} \sup _{\left[t_{j}, t_{j+1}\right]}\left\|\left(R_{h}-1\right) u^{\prime}(t)\right\|_{0}^{2}+\sum_{j \in n}(\Delta t)^{2} \sup _{\left[t_{j}, t_{j+1}\right]}\left\|u^{\prime \prime}(t)\right\|_{0}^{2}\right) \\
\leqslant & \left\|\sigma^{0}\right\|_{0}^{2}+C \frac{\Delta t}{\varepsilon} h^{2} \sum_{j \in n}\left\|f^{j+\theta}\right\|_{0}^{2} \\
& +C \frac{T}{\varepsilon}\left(1+h^{2}\right)\left(\sup _{[0, T]}\left\|\left(R_{h}-1\right) u^{\prime}(t)\right\|_{0}^{2}+(\Delta t)^{2} \sup _{[0, T]}\left\|u^{\prime \prime}(t)\right\|_{0}^{2}\right)
\end{aligned}
$$

mit einer Konstante, die weder von $h$, von $\Delta t$ noch von den Daten des Problems (3.26) abhängt. Anwendung der Dreieckungleichung auf $u^{n}-u_{h}^{n}=\rho^{n}+\sigma^{n}$ führt auf die Ungleichung

$$
\begin{aligned}
\left\|u\left(t_{n}\right)-u_{h}^{n}\right\|_{0}^{2} \leqslant & C\left(\left\|\left(R_{h}-1\right) u_{0}\right\|_{0}^{2}+\left\|\left(R_{h}-1\right) u\left(t_{n}\right)\right\|_{0}^{2}+\frac{\Delta t}{\varepsilon} h^{2} \sum_{j \in n}\left\|f^{j+\theta}\right\|_{0}^{2}\right) \\
& +C \frac{T}{\varepsilon}\left(1+h^{2}\right)\left(\sup _{[0, T]}\left\|\left(R_{h}-1\right) u^{\prime}(t)\right\|_{0}^{2}+(\Delta t)^{2} \sup _{[0, T]}\left\|u^{\prime \prime}(t)\right\|_{0}^{2}\right) .
\end{aligned}
$$

Der Beweis wird komplettiert durch Abschätzung der Terme, die $R_{h}-1$ enthalten, mit Hilfe von Lemma 3.3.1 unter Berücksichtigung der Regularitätsforderungen an das adjungierte Problem (3.15) sowie an $u, u^{\prime}$ und $u^{\prime \prime}$.

Bemerkung 3.4.4 (i) Für das Crank-Nicolson-Verfahren, d. h. für $\theta=\frac{1}{2}$, läßt sich eine Abschätzung der Gestalt (3.31) bzw. (3.32) beweisen, die statt des Terms $(\Delta t)^{2} \sup _{[0, T]}\left\|u^{\prime \prime}(t)\right\|_{0}^{2}$ 
den Term $(\Delta t)^{4} \sup _{[0, T]}\left\|u^{\prime \prime \prime}(t)\right\|_{0}^{2}$ aufweist bei entsprechender Regularität von $u$. Dies bedeutet die Konvergenzordnung $O\left((\Delta t)^{2}\right)$ bezüglich der zeitlichen Diskretisierung.

(ii) Analog zu Bemerkung 3.4.2 gelangt man für $\theta \geqslant \frac{1}{2} \mathrm{zu}$ einer verbesserten, mit einem dämpfenden Faktor gewichteten Konvergenzabschätzung. 


\section{Kapitel 4}

\section{Stabilisierte Finite-Volumen-Verfahren}

Bei der bisher vorgestellten Finite-Volumen-Diskretisierung, einer „unstabilisierten“ Methode, treten für $\varepsilon \rightarrow 0$ die im Abschnitt 1.1 im Zusammenhang mit singulär gestörten Problemen geschilderten Unzulänglichkeiten auf. Dies ergibt sich schon aus dem Umstand, daß sich diese Diskretisierung als verallgemeinertes Galerkin-Verfahren interpretieren läßt. Wir werden deshalb in diesem Kapitel ein stabilisiertes Finite-Volumen-Verfahren beschreiben und untersuchen.

Nach der Konstruktion eines Upwind-Finite-Volumen-Verfahrens zu Beginn zeigen wir dessen Stabilität und zwar unabhängig von der Stärke der Diffusion. Anschließend untersuchen wir den Konsistenzfehlers des Verfahrens und leiten daraus a-priori-Fehlerabschätzungen ab. Dabei greifen wir auf Ergebnissen zurück, die in Kapitel 3 erzielt wurden.

Der Einfachheit halber beschränken wir uns in diesem Kapitel auf Probleme mit $\Omega \subseteq \mathbb{R}^{2}$, sämtliche Ergebnisse sind jedoch problemlos auf Fälle im $\mathbb{R}^{3}$ übertragbar. Als duale Volumina der Diskretisierung werden die des Donald-Diagramms dienen.

\subsection{Upwind-Modifikation}

Zunächst erinnern wir an die betrachtete Klasse von zeitabhängigen Konvektions-DiffusionsReaktions-Problemen:

Finde $u \in H^{1}(0, T ; V), V=H_{0}^{1}(\Omega)$, mit

$$
\left\{\begin{aligned}
\frac{d}{d t}(u(t), v)+a(u(t), v) & =(f(t), v) \quad \text { für fast alle } t \in(0, T) \text { und alle } v \in V \\
u(0) & =u_{0} \in L^{2}(\Omega)
\end{aligned}\right.
$$

wobei die Bilinearform die Gestalt

$$
a(u, v)=\int_{\Omega} \nabla v \cdot(A \nabla u-u b)+c u v d x
$$


hat und die Daten den Bedingungen

$$
a_{i j}, b_{j}, c, \nabla \cdot b \in L^{\infty}(\Omega) \text { und } a_{i j}=a_{j i} \text { für jedes } i, j \in \underline{d}, f \in L^{2}\left(Q_{T}\right)
$$

sowie

$$
\eta:=\underset{\Omega}{\operatorname{essinf}} q>0 \text { mit } q:=\frac{1}{2} \nabla \cdot b+c, \quad \varepsilon:=\underset{\Omega}{\operatorname{essinf}}\left\{\frac{y \cdot A y}{|y|^{2}} \mid y \in \mathbb{R}^{d} \backslash\{0\}\right\}>0
$$

genügen.

Prinzipiell orientieren wir uns bei der Konstruktion eines upwind-stabilisierten FiniteVolumen-Verfahrens und dessen Analyse am Vorgehen in [4] sowie in [41], Kapitel 6. Dazu sei $\left(\mathcal{T}_{h}\right)_{h>0}$ eine Familie von Triangulierungen auf $\Omega$ gemäß (TR) (siehe Abschnitt 2.1). Sofern aus dem Kontext die Zugehörigkeit zu einer Triangulierung $\mathcal{T}_{h}$ ersichtlich ist, bezeichnet $\left\{a_{1}, \ldots, a_{N_{h}}\right\}$ die Knoten und $\mathcal{B}_{h}^{D}=\left\{B_{1}, \ldots, B_{N_{h}}\right\}$ das Donald-Diagramm zu $\mathcal{T}_{h}$. Wie im Abschnitt 2.1 vereinbart, besteht $\left\{a_{1}, \ldots, a_{N_{h, 0}}\right\}$ dann aus den inneren Knoten.

Die Bilinearform der daraus resultierenden Finite-Volumen-Diskretisierung (vergl. (2.4)(2.6)) besitzt die Darstellung

$$
a_{h}(u, v)=d_{h}(u, v)+c_{h}(u, v)+r_{h}(u, v), \quad u, v \in P^{1}\left(\mathcal{T}_{h}\right),
$$

mit

$$
\begin{aligned}
d_{h}(u, v) & :=-\sum_{i \in \underline{N_{h}}} v\left(a_{i}\right) \int_{\partial B_{i} \backslash \partial \Omega} \bar{A} \nabla u \cdot d s, \\
c_{h}(u, v) & :=\sum_{i \in \underline{N_{h}}} v\left(a_{i}\right) \int_{\partial B_{i}} b u \cdot d s, \\
r_{h}(u, v) & :=\sum_{i \in \underline{N_{h}}} v\left(a_{i}\right) \int_{B_{i}} c u d x .
\end{aligned}
$$

Zu $i \in N_{h}$ bezeichne $I_{i}$ die Menge der Indizes aller benachbarten Knoten

$$
I_{i}:=\left\{j \in N_{h} \backslash\{i\} \mid B_{i} \cap B_{j} \neq \emptyset\right\} .
$$

Sind $a_{i}, a_{j}$ zwei benachbarte Knoten der Triangulierung $\mathcal{T}_{h}$, so sei $\Gamma_{i j}$ der gemeinsame Rand der zugehörigen dualen Volumina, und für jeden Randknoten $a_{i} \in \Gamma$ sei $\Gamma_{i}$ der Randabschnitt des zugehörigen dualen Volumens auf $\partial \Omega$, also

$$
\Gamma_{i j}:=B_{i} \cap B_{j}, \quad \Gamma_{i}:=B_{i} \cap \partial \Omega .
$$

Im Fall einer stetigen Funktion $w$ verwenden wir außerdem die abkürzende Schreibweise $w_{i}$ anstatt des Wertes $w\left(a_{i}\right)$ am Knoten $a_{i}$.

Für den Konvektionsterm $c_{h}(u, v)$ gilt mit den soeben eingeführten Bezeichnungen offensichtlich

$$
c_{h}(u, v)=\sum_{i \in \underline{N_{h}}} v_{i} \int_{\partial B_{i}} b u \cdot d s=\sum_{i \in \underline{N_{h}}} v_{i} \sum_{j \in I_{i}} \int_{\Gamma_{i j}} b u \cdot d s+\sum_{i>N_{h, 0}} v_{i} \int_{\Gamma_{i}} b u \cdot d s .
$$

Zur upwind-Modifikation ersetzen wir $u$ auf jedem Randstück $\Gamma_{i j}$ durch eine Konvexkombination der Knotenwerte $u_{i}$ und $u_{j}$. Die Koeffizienten werden dabei durch eine upwind-Funktion bestimmt, d. h. durch eine Abbildung $\lambda: \mathbb{R} \rightarrow[0,1]$, die die Eigenschaften 
( $\lambda 1) \quad \lambda(x)=1-\lambda(-x) \quad$ für alle $x>0$,

$(\lambda 2) \lambda(x) \geqslant \frac{1}{2} \quad$ für alle $x \geqslant 0$

trägt. Beispiele, die in der Literatur Anwendung finden, und deren Bezeichnungen sind etwa (siehe [41] und [59] sowie die dort zitierte Literatur)

$$
\begin{aligned}
& \lambda(x)=\lambda_{f}(x)=\frac{1}{2}(\operatorname{sign}(x)+1) \quad \text { volles Upwinding } \\
& \lambda(x)=\lambda_{s}(x)=\frac{1}{2}\left(\frac{x}{1+|x|}+1\right) \quad \text { Samarskij Upwinding } \\
& \lambda(x)=\lambda_{e}(x)=\left\{\begin{array}{ll}
1-\frac{1}{x}\left(1-\frac{x}{e^{x}-1}\right), & \text { falls } x \neq 0, \\
\frac{1}{2}, & \text { falls } x=0
\end{array} \quad\right. \text { exponentielles Upwinding }
\end{aligned}
$$

Abbildung 4.1 zeigt die drei genannten Funktionen. Die Wahl der upwind-Funktion bietet Kontrolle über die numerische Viskosität, die durch die Stärke des Upwindings, also des dominierenden Einfluß' der Werte in stromaufwärts liegen Punkten gegenüber stromabwärts liegenden, bestimmt wird.

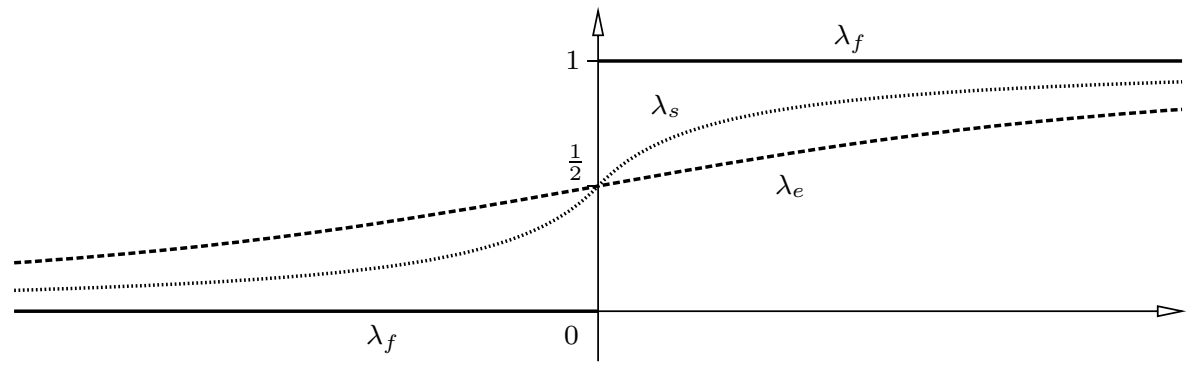

Abbildung 4.1: Die upwind-Funktionen $\lambda_{f}, \lambda_{s}$ und $\lambda_{e}$

Daneben sei $\beta_{i j} \in \mathbb{R}$ zu jedem Indexpaar $(i, j) \in \underline{N_{h}} \times \underline{N_{h}}$ mit $B_{i} \cap B_{j} \neq \emptyset$ eine Approximation von $\int_{\Gamma_{i j}} b \cdot d s$ derart, daß

$$
\begin{aligned}
& \left|\sum_{j \in I_{i}}\left(\int_{\Gamma_{i j}} b \cdot d s-\beta_{i j}\right)\right| \leqslant C h\left|B_{i}\right|, \quad i \in N_{h}, \\
& \beta_{i j}+\beta_{j i}=0
\end{aligned}
$$

mit einer von $h$ (und $b$ ) unabhängigen Konstante $C>0$ erfüllt ist.

Wir ersetzen nun $\left.u\right|_{\Gamma_{i j}} \operatorname{durch} \lambda_{i j} u_{i}+\left(1-\lambda_{i j}\right) u_{j}$ mit

$$
\lambda_{i j}:=\lambda\left(\frac{\beta_{i j}}{\varepsilon}\right)
$$

sowie $\left.u\right|_{\Gamma_{i}}$ durch $u_{i}$ und gelangen insgesamt so zu 


$$
c_{h}^{u p}(u, v):=\sum_{i \in \underline{N_{h}}} v_{i} \sum_{j \in I_{i}}\left(u_{i} \lambda_{i j}+\left(1-\lambda_{i j}\right) u_{j}\right) \beta_{i j}+\sum_{i>N_{h, 0}} v_{i} u_{i} \int_{\Gamma_{i}} b \cdot d s
$$

als upwind-Modifikation von $c_{h}(u, v)$.

Man beachte, wegen der Bedingungen $(\lambda 1)$ und $(\lambda 2)$ folgt $\lambda(x) \leqslant \frac{1}{2}$ für alle $x<0$, berücksichtigt man zudem (4.6), so folgt $\lambda_{i j}=1-\lambda_{j i}$. Anhand dieser Eigenschaften von $\lambda$ bzw. $\lambda_{i j}$ und mit (4.6) zeigt man die folgende Abschätzung für $c_{h}^{u p}(v, v)$, die wesentlich die Stabilität des Verfahrens begründen wird.

Lemma 4.1.1 Für jede Funktion $v \in P^{1}\left(\mathcal{T}_{h}\right)$ gilt

$$
c_{h}^{u p}(v, v) \geqslant \frac{1}{2} \sum_{i \in N_{h}} \sum_{j \in I_{i}} v_{i}^{2} \beta_{i j}+\sum_{i>N_{h, 0}} v_{i} u_{i} \int_{\Gamma_{i}} b \cdot d s .
$$

Beweis. $\quad$ Vergl. Beweis zu Lemma 6.12 in [41] und nutze $\left(\lambda_{i j}-\frac{1}{2}\right) \beta_{i j} \geqslant 0$.

Den Reaktionsterm $r_{h}(u, v)$ ändern wir ab zu

$$
r_{h}^{u p}(u, v):=\sum_{i \in \underline{N_{h}}} \gamma_{i} u_{i} v_{i}
$$

d. h. die Ansatzfunktion $u$ wird ebenfalls auf $P^{0}\left(\mathcal{T}_{h}\right)$ projeziert (mass lumping), wodurch der Anteil der Reaktion an der Steifigkeitsmatrix ausschließlich Diagonaleinträge liefert. Außerdem wird jedes der Integrale $\int_{B_{i}} c d x$ durch $\gamma_{i} \in \mathbb{R}$ approximiert und zwar von der Güte

$$
\left|\int_{B_{i}} c d x-\gamma_{i}\right| \leqslant C h\left|B_{i}\right|, \quad i \in N_{h} .
$$

mit einer von $h$ (und $c$ ) unabhängigen Konstante $C>0$.

Auf diese Weise gelangt man schließlich $\mathrm{zu}$ einem upwind-stabilisierten Finite-VolumenVerfahren zum Problem (4.1)-(4.4) bezüglich der Triangulierung $\mathcal{T}_{h}$ und deren DonaldDiagramms $\mathcal{B}_{h}^{D}$ :

Finde $u_{h} \in H^{1}\left(0, T ; V_{h}\right), V_{h}=P_{0}^{1}\left(\mathcal{T}_{h}\right)$, so daß

$$
\left\{\begin{aligned}
\frac{d}{d t}\left(u_{h}(t), v\right)+a_{h}^{u p}\left(u_{h}(t), v\right) & =\left\langle F_{h}(t), v\right\rangle \quad \text { für fast alle } t \in(0, T) \text { und alle } v \in V_{h} \\
u_{h}(0) & =u_{0, h} \in V_{h}
\end{aligned}\right.
$$

mit der Bilinearform

$$
a_{h}^{u p}(u, v):=d_{h}(u, v)+c_{h}^{u p}(u, v)+r_{h}^{u p}(u, v)
$$

und der Linearform gemäß (2.6)

$$
\left\langle F_{h}(t), v\right\rangle=\int_{\Omega} f(t) \hat{v} d x=\sum_{i \in \underline{N_{h}}} v\left(a_{i}\right) \int_{B_{i}} f(t) d x .
$$


Wir geben noch eine geeignete Realisierung der approximierenden Größen $\beta_{i j}$ und $\gamma_{i}$ an. Zu diesem Zweck führen wir einige weitere Bezeichnungen ein. Es sei $a_{\Gamma_{i j}}$ der Mittelpunkt der Kante zwischen zwei Knoten $a_{i}, a_{j}$. Für jedes Element $T \in \mathcal{T}_{h}$ mit $a_{i}, a_{j} \in T$ sei außerdem $\Gamma_{i j}(T):=\Gamma_{i j} \cap T$ und

$$
B_{i j}(T):=\operatorname{conv}\left(\left\{a_{i}, a_{\Gamma_{i j}}, \sigma(T)\right\}\right),
$$

also das Dreieck mit den Eckpunkten $a_{i}, a_{\Gamma_{i j}}$ und $\sigma(T)$. Nach Konstruktion des DonaldDiagramms ist $a_{\Gamma_{i j}} \in \Gamma_{i j}$ und $\Gamma_{i j}(T)$ eine Kante von $B_{i j}(T)$.

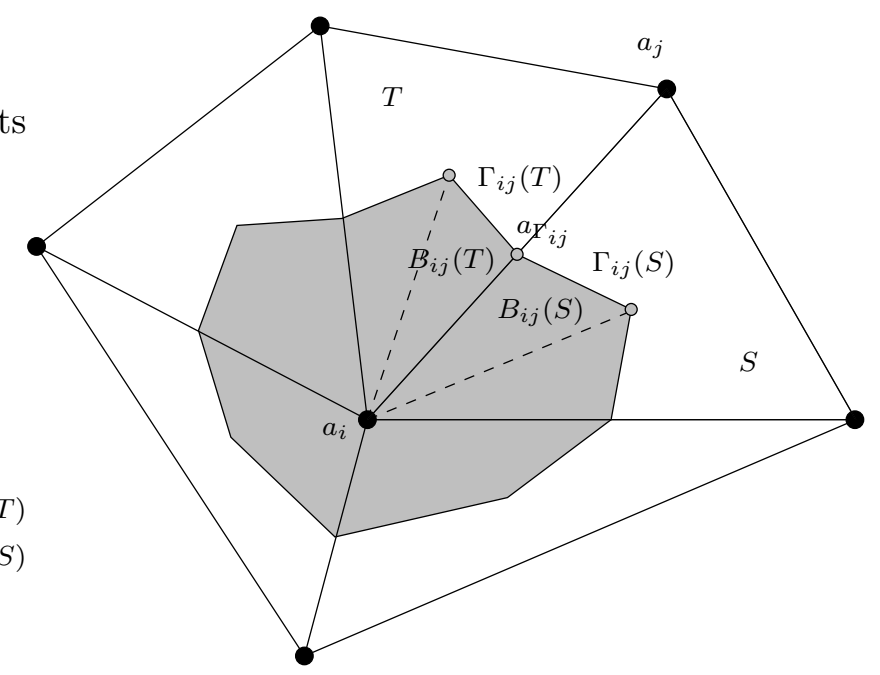

Abbildung 4.2: Zur Definition von $a_{\Gamma_{i j}}, \Gamma_{i j}(T)$ und $B_{i j}(T)$

Ist $c \in H^{1, \infty}(\Omega)$ und $b \in H^{2, \infty}(\Omega)$, so sind nach Satz A.2.2 $c$ und $b$ stetig, und es kann ersetzt werden

$$
\int_{\Gamma_{i j}} b \cdot d s \quad \leadsto \quad \beta_{i j}=\int_{\Gamma_{i j}} b\left(a_{\Gamma_{i j}}\right) \cdot d s
$$

und

$$
\int_{B_{i}} c d x \quad \leadsto \quad \gamma_{i}=\int_{B_{i}} L_{h} c d x=c_{i}\left|B_{i}\right| .
$$

Offensichtlich ist dann $\beta_{i j}=-\beta_{j i}$, und nach Lemma 3 in [4] gilt für $T \in \mathcal{T}_{h}$ mit $a_{i}, a_{j} \in T$

$$
\left|\int_{\Gamma_{i j}(T)} b \cdot d s-\beta_{i j}\right| \leqslant C h_{B_{i j}(T)}^{2}|b|_{2, \infty}
$$

mit einer von $h$ und $b$ unabhängigen Konstante $C>0$. Dabei bezeichnet $h_{B_{i j}(T)}$ den Durchmesser von $B_{i j}(T)$.

Wegen der Regularität der Triangulierungen gilt eine Minimalwinkelbedingung (siehe etwa Abschnitt 3.4.1 in [41], beachte $\Omega \subseteq \mathbb{R}^{2}$ ). Eine solche gilt dann auch für die Teilwinkel, die 
beim Donald-Diagramm auftreten, also die Innenwinkel eines beliebigen $B_{i j}(T)$. Es sei $\psi_{\text {sup }}$ Supremum dieser Innenwinkel gleichmäßig in $h$. Dann gilt

$$
\left|\Gamma_{i j}(T)\right| h_{B_{i j}(T)} \leqslant 4 \cot \psi_{\text {sup }}\left|B_{i j}(T)\right|,
$$

also ist die Bedingung (4.5) erfüllt.

Ferner gilt nach Lemma 14 in [6]

$$
\left\|c-L_{h} c\right\|_{\infty} \leqslant C h|c|_{1, \infty}
$$

mit einer von $h$ und $c$ unabhängigen Konstante $C>0$. Somit folgt

$$
\left|\int_{B_{i}} c-L_{h} c d x\right| \leqslant\left\|c-L_{h} c\right\|_{\infty} \int_{B_{i}} d x \leqslant C h|c|_{1, \infty}\left|B_{i}\right|
$$

für jedes $i \in \underline{N_{h}}$, wodurch die Bedingung (4.9) befriedigt wird.

\subsection{Stabilität}

Im Abschnitt 3.2 wurde die $V_{h}$-Koerzivität, $V_{h}=P_{0}^{1}\left(\mathcal{T}_{h}\right)$, von $a_{h}$ nachgewiesen (bezüglich der Norm $\|\cdot\|_{\varepsilon}$ ) für hinreichend kleine $h>0$. Die Beschränkung für $h$ hing jedoch ungünstig von $\varepsilon$ ab. Im Gegensatz dazu kann hier die $V_{h}$-Koerzivität der Bilinearform $a_{h}^{u p}$ unterhalb eines $h_{0}$ unabängig von $\varepsilon$ nachgewiesen werden.

Weiterhin sei $\left(\mathcal{T}_{h}\right)_{h>0}$ eine Familie von Triangulierungen auf $\Omega$ gemäß (TR) und $\mathcal{B}_{h}^{D}=$ $\left\{B_{1}, \ldots, B_{N_{h}}\right\}$ das Donald-Diagramm zu $\mathcal{T}_{h}$. Zudem finden die im letzten Abschnitt eingeführten Bezeichnungen Verwendung. Wir zitieren zunächst das folgende Lemma, um anschließend die Koerzivitätsaussage zeigen können.

Lemma 4.2.1 Es gibt eine von $h$ unabhängige Konstante $C_{0}>0$, so daß

$$
\sum_{i \in \underline{N_{h}}} v_{i}^{2}\left|B_{i}\right| \geqslant C_{0}\|v\|_{0}^{2}
$$

für jedes $v \in P^{1}\left(\mathcal{T}_{h}\right)$ gilt.

Beweis. $\quad$ Vergl. Bemerkung 6.16 in [41].

Satz 4.2.2 Es seien die Bedingungen (4.3) sowie (4.4) vorausgesetzt. Für $\beta_{i j}$ und $\gamma_{i}$ gelte (4.5)-(4.6) bzw. (4.9). Ferner seien die Koeffizienten $\lambda_{i j}$ laut (4.7) gewählt mit einer Funktion $\lambda \in[0,1]^{\mathbb{R}}$, die den Bedingungen $(\lambda 1)$ und $(\lambda 2)$ genügt. Dann existiert eine von $\varepsilon$ unabhängige Konstante $h_{0}>0$, so daß für alle $h \in\left(0, h_{0}\right]$ gilt

$$
a_{h}^{u p}(v, v) \geqslant \alpha_{u p}\|v\|_{\varepsilon}^{2} \quad \text { für jedes } v \in V_{h}
$$

mit der Koerzivitätskonstante $\alpha_{u p}:=\min \left\{1, \frac{\eta}{2\|q\|_{\infty}} C_{0}\right\}$, die weder von $h$ noch von $\varepsilon$ abhängt. 
Beweis. $\quad$ Es sei $v \in V_{h}$. Dann ist (siehe Lemma 3.1.1)

$$
d_{h}(v, v)=\left\|A^{\frac{1}{2}} \nabla v\right\|_{0}^{2}
$$

und wegen (4.8), (4.4) sowie $\int_{B_{i}} \nabla \cdot b d x=\sum_{j \in I_{i}} \int_{\Gamma_{i j}} b \cdot d s$ folgt

$$
\begin{aligned}
c_{h}^{u p}(v, v)+r_{h}^{u p}(v, v) \geqslant & \frac{1}{2} \sum_{i \in N_{h, 0}} \sum_{j \in I_{i}} v_{i}^{2} \beta_{i j}+\sum_{i \in N_{h, 0}} \gamma_{i} v_{i}^{2} \\
= & \sum_{i \in N_{h, 0}} v_{i}^{2}\left(\int_{B_{i}} \frac{1}{2} \nabla \cdot b+c d x-\frac{1}{2} \sum_{j \in I_{i}}\left(\int_{\Gamma_{i j}} b \cdot d s-\beta_{i j}\right)\right. \\
& \left.\quad-\int_{B_{i}} c d x+\gamma_{i}\right) \\
\geqslant & \sum_{i \in \underline{N_{h, 0}}} v_{i}^{2}\left(\left|B_{i}\right| \eta-\frac{1}{2}\left|\sum_{j \in I_{i}}\left(\int_{\Gamma_{i j}} b \cdot d s-\beta_{i j}\right)\right|-\left|\int_{B_{i}} c d x-\gamma_{i}\right|\right) .
\end{aligned}
$$

Mit Hilfe von (4.5) und (4.9) folgert man daraus

$$
\begin{aligned}
c_{h}^{u p}(v, v)+r_{h}^{u p}(v, v) & \geqslant \sum_{i \in N_{h, 0}} v_{i}^{2}\left|B_{i}\right|(\eta-C h) \\
& \geqslant \frac{1}{2} \eta \underline{\sum_{i \in N_{h, 0}}} v_{i}^{2}\left|B_{i}\right|
\end{aligned}
$$

für $h \leqslant h_{0}=\frac{\eta}{2 C}$. Zieht man Lemma 4.2.1 heran, so ergibt sich daher mit dieser Beschränkung an $h$

$$
a_{h}^{u p}(v, v) \geqslant\left\|A^{\frac{1}{2}} \nabla v\right\|_{0}^{2}+\frac{1}{2} \eta C_{0}\|v\|_{0}^{2} \geqslant\left\|A^{\frac{1}{2}} \nabla v\right\|_{0}^{2}+\frac{\eta}{2\|q\|_{\infty}} C_{0}\|\sqrt{q} v\|_{0}^{2},
$$

wobei wieder $q=\frac{1}{2} \nabla \cdot b+c$. Dies liefert mit $\alpha_{u p}:=\min \left\{1, \frac{\eta}{2\|q\|_{\infty}} C_{0}\right\}$ die Behauptung.

Der Beweis kommt ohne Konsistenzfehlerabschätzung für $a_{h}-a_{h}^{u p}$ aus, die Gegenstand des nächsten Abschnitts sein wird.

Bemerkung 4.2.3 Die zusätzliche Einschränkung an $h$ durch ein $h_{0}$ geht auf die „Quadraturen" $\beta_{i j}$ und $\gamma_{i}$ zurück und nicht auf die eigentliche upwind-Modifikation. Trivialerweise erhält man nämlich mit der Wahl $\beta_{i j}=\frac{1}{\left|\Gamma_{i j}\right|} \int_{\Gamma_{i j}} b \cdot d s$ und $\gamma_{i}=\frac{1}{\left|B_{i}\right|} \int_{B_{i}} c d x$ exakt die Integrale $\int_{\Gamma_{i j}} b \cdot d s$ bzw. $\int_{B_{i}} c d x$ zurück. In diesem Fall gilt die Aussage von Satz 4.2 .2 ohne Beschränkung an $h$ nach oben.

Völlig analog zum Beweis von (3.17) in Lemma 3.3.2 zeigt man jetzt die folgende exponentiell gewichtete a-priori-Abschätzung. 
Lemma 4.2.4 Es seien die Voraussetzungen von Lemma 2.2.2 und Satz 4.2.2 erfüllt. Dann genügt eine Lösung $u_{h} \in H^{1}\left(0, T ; V_{h}\right)$ des Problems (4.10) - (4.12) für fast alle $t \in(0, T)$ und alle $\lambda \in\left[0, \eta \alpha_{u p}\right)$ der Abschätzung

$$
\left\|u_{h}(t)\right\|_{0}^{2}+\left(\alpha_{u p}-\frac{\lambda}{\eta}\right) \int_{0}^{t} e^{2 \lambda(s-t)}\left\|u_{h}(s)\right\|_{\varepsilon}^{2} d s \leqslant e^{-2 \lambda t}\left\|u_{0, h}\right\|_{0}^{2}+\frac{C_{d}}{\eta \alpha_{u p}-\lambda} \int_{0}^{t} e^{2 \lambda(s-t)}\|f(s)\|_{0}^{2} d s
$$

mit $C_{d}:=(d+1)(d+2)$.

Man beachte, daß im Gegensatz zu Lemma 3.3.2 hier keine Beschränkung an $h$ in Abhängigkeit von $\varepsilon$ benötigt wird.

\subsection{Konsistenzfehlerabschätzung}

Für das vorgestellte upwind-stabilisierte Finite-Volumen-Verfahren leiten wir nun eine Abschätzung des Konsistenzfehlers her. Nach den Ergebnissen aus dem Abschnitt 2.3 wird dazu nur noch der Fehler, der durch die Stabilisierung entsteht, zu betrachten sein.

Das folgende Lemma liefert Abschätzungen für die Differenz der Konvektionsterme $c_{h}(u, v)$ und $c_{h}^{u p}(u, v)$ sowie der Reaktionsterme $r_{h}(u, v)$ und $r_{h}^{u p}(u, v)$. Sowohl in der Bilinearform des Finite-Volumen-Verfahrens als auch in der des stabilisierten Verfahrens tritt die Testfunktion $v \in P^{1}\left(\mathcal{T}_{h}\right)$ als Transformierte $\hat{v}$ auf. Bei der Abschätzung der Differenzen wird daher die Abschätzung zwischen $\|\hat{v}\|_{0}$ und $\|v\|_{0}$ durch Lemma 2.2 .2 nützlich sein.

Lemma 4.3.1 Es sei $\Omega \subseteq \mathbb{R}^{2}$ ein polyedrisches Lipschitz-Gebiet und $\mathcal{T}_{h}$ eine zulässige durch affine Transformation von einem Referenzelement erzeugte Triangulierungen darauf sowie $\mathcal{B}_{h}^{D}$ das Donald-Diagramm zu $\mathcal{T}_{h}$. Für je zwei Funktionen $u, v \in P^{1}\left(\mathcal{T}_{h}\right)$ gilt dann

$$
\left|c_{h}(u, v)-c_{h}^{u p}(u, v)\right| \leqslant C h\left(\|u\|_{0}\|v\|_{0}+\|b\|_{1, \infty}|u|_{1}\left(|v|_{1}+\|v\|_{0}\right)\right),
$$

falls $b \in\left(H^{1, \infty}(\Omega)\right)^{2}$ und

$$
\left|r_{h}(u, v)-r_{h}^{u p}(u, v)\right| \leqslant C h\left(\|c\|_{\infty}|u|_{1}+|c|_{1, \infty}\|u\|_{0}\right)\|v\|_{0}
$$

falls $c \in H^{1, \infty}(\Omega)$, jeweils mit einer Konstante $C>0$, die weder von $h$ noch von $b$ oder $c$ abängt.

Beweis. Um auf die Fehlerabschätzungen aus [4] und [6] zurückgreifen zu können, zerlegen wir $c_{h}^{u p}$ zunächst wie folgt.

$$
\begin{aligned}
c_{h}^{u p}(u, v) & =\sum_{i \in \underline{N_{h}}} v_{i} \sum_{j \in I_{i}}\left(1-\lambda_{i j}\right)\left(u_{j}-u_{i}\right) \beta_{i j}+\sum_{i \in \underline{N_{h}}} v_{i} \sum_{j \in I_{i}} u_{i} \beta_{i j}+\sum_{i>N_{h, 0}} v_{i} \int_{\Gamma_{i}} b u \cdot d s \\
& =S^{(0)}+\frac{1}{2} \sum_{i \in \underline{N_{h}}} v_{i} \sum_{j \in I_{i}}\left(u_{j}-u_{i}\right) \beta_{i j}+\underline{\sum_{i \in \underline{N_{h}}}} v_{i} \sum_{j \in I_{i}} u_{i} \beta_{i j}+\sum_{i>N_{h, 0}} v_{i} \int_{\Gamma_{i}} b u \cdot d s
\end{aligned}
$$


mit

$$
S^{(0)}:=\sum_{i \in \underline{N_{h}}} v_{i} \sum_{j \in I_{i}}\left(\frac{1}{2}-\lambda_{i j}\right)\left(u_{j}-u_{i}\right) \beta_{i j} .
$$

Für die zweite Doppelsumme der rechten Seite von (4.19) ergibt sich darüber hinaus

$$
\begin{aligned}
\frac{1}{2} \sum_{i \in \underline{N_{h}}} v_{i} \sum_{j \in I_{i}}\left(u_{j}-u_{i}\right) \beta_{i j}= & S^{(1)}+\frac{1}{2} \sum_{i \in \underline{N_{h}}} v_{i} \sum_{j \in I_{i}}\left(u_{j}-u_{i}\right) \int_{\Gamma_{i j}} b \cdot d s \\
= & S^{(1)}+\sum_{i \in \underline{N_{h}}} v_{i} \sum_{j \in I_{i}} \int_{\Gamma_{i j}}\left(\frac{1}{2}\left(u_{j}+u_{i}\right)-u\right) b \cdot d s \\
& +\sum_{i \in \underline{N_{h}}} v_{i} \sum_{j \in I_{i}} \int_{\Gamma_{i j}}\left(u-u_{i}\right) b \cdot d s
\end{aligned}
$$

mit

$$
S^{(1)}:=\frac{1}{2} \sum_{i \in \underline{N_{h}}} v_{i} \sum_{j \in I_{i}}\left(u_{j}-u_{i}\right)\left(\beta_{i j}-\int_{\Gamma_{i j}} b \cdot d s\right) .
$$

Setzt man zudem

$$
S^{(2)}:=\sum_{i \in \underline{N_{h}}} v_{i} \sum_{j \in I_{i}} \int_{\Gamma_{i j}}\left(\frac{1}{2}\left(u_{j}+u_{i}\right)-u\right) b \cdot d s
$$

so erhält man wegen

$$
\sum_{j \in I_{i}} \int_{\Gamma_{i j}}\left(u-u_{i}\right) b \cdot d s=\int_{B_{i}} \nabla \cdot(u b) d x-\int_{\Gamma_{i}} b u \cdot d s-u_{i} \sum_{j \in I_{i}} \int_{\Gamma_{i j}} b \cdot d s
$$

insgesamt für $c_{h}^{u p}$ daher die Darstellung

$$
c_{h}^{u p}(u, v)=\int_{\Omega} \hat{v} \nabla \cdot(u b) d x+\sum_{i \in \underline{N_{h}}} u_{i} v_{i} \sum_{j \in I_{i}}\left(\beta_{i j}-\int_{\Gamma_{i j}} b \cdot d s\right)+S^{(0)}+S^{(1)}+S^{(2)} .
$$

Für den Fehler beim Übergang von $c_{h}(u, v)$ zu $c_{h}^{u p}(u, v)$ bedeutet dies

$$
\left|c_{h}(u, v)-c_{h}^{u p}(u, v)\right| \leqslant \sum_{i \in \underline{N_{h}}}\left|u_{i}\right|\left|v_{i}\right|\left|\sum_{j \in I_{i}}\left(\beta_{i j}-\int_{\Gamma_{i j}} b \cdot d s\right)\right|+\sum_{k \in 3}\left|S^{(k)}\right| .
$$

Unter Ausnutzung von (4.5) und der Cauchy-Schwarzschen Ungleichung folgert man zum einen

$$
\begin{aligned}
\sum_{i \in N_{h}}\left|u_{i}\right|\left|v_{i}\right|\left|\sum_{j \in I_{i}}\left(\beta_{i j}-\int_{\Gamma_{i j}} b \cdot d s\right)\right| & \leqslant C h \sum_{i \in \underline{N_{h}}}\left|u_{i}\right|\left|v_{i}\right|\left|B_{i}\right| \\
& \leqslant C h\left(\sum_{i \in N_{h}} u_{i}^{2}\left|B_{i}\right|\right)^{\frac{1}{2}}\left(\sum_{i \in N_{h}} v_{i}^{2}\left|B_{i}\right|\right)^{\frac{1}{2}} \\
& =C h\|\hat{u}\|_{0}\|\hat{v}\|_{0} .
\end{aligned}
$$


Zum anderen entnimmt man dem Beweis von Lemma 24 in [6] (siehe auch Beweis zu Lemma 7 in [4] im Fall eines Voronoi-Diagramms) zur Abschätzung der Summanden $S^{(k)}, k \in 3$,

$$
\begin{aligned}
& \left|S^{(0)}\right| \leqslant C h\|b\|_{1, \infty}|u|_{1}|v|_{1}, \\
& \left|S^{(1)}\right| \leqslant C h\|\nabla \cdot b\|_{\infty}|u|_{1}\|\hat{v}\|_{0}, \\
& \left|S^{(2)}\right| \leqslant C h\|b\|_{1, \infty}|u|_{1}|v|_{1}
\end{aligned}
$$

für je zwei Funktionen $u, v \in P^{1}\left(\mathcal{T}_{h}\right)$, jeweils mit einer Konstante $C>0$, die weder von $h$ noch von $b$ abhängt. Zusammenfassend gilt also

$$
\left|c_{h}(u, v)-c_{h}^{u p}(u, v)\right| \leqslant C h\left(\|\hat{u}\|_{0}\|\hat{v}\|_{0}+\|b\|_{1, \infty}|u|_{1}|v|_{1}+\|\nabla \cdot b\|_{\infty}|u|_{1}\|\hat{v}\|_{0}\right) .
$$

Als nächstes gehen wir auf die Differenz $r_{h}(u, v)-r_{h}^{u p}(u, v)$ ein. Es ist

$$
\int_{\Omega} c u \hat{v} d x-\sum_{j \in \underline{N_{h}}} c_{i} \hat{u}_{i} \hat{v}_{i} d x=\int_{\Omega} \hat{c}(u-\hat{u}) \hat{v} d x+\int_{\Omega}(c-\hat{c}) u \hat{v} d x .
$$

Mit Lemma 2.2.1 und (4.15) folgt daher

$$
\begin{aligned}
\left|r_{h}(u, v)-r_{h}^{u p}(u, v)\right| & \leqslant\|c\|_{\infty}\|u-\hat{u}\|_{0}\|\hat{v}\|_{0}+\|c-\hat{c}\|_{\infty}\|u\|_{0}\|\hat{v}\|_{0} \\
& \leqslant\left(h\|c\|_{\infty}|u|_{1}+C h|c|_{1, \infty}\|u\|_{0}\right)\|\hat{v}\|_{0} .
\end{aligned}
$$

Nach Lemma 2.2.2 ist

$$
\|\hat{v}\|_{0} \leqslant \sqrt{12}\|v\|_{0}
$$

für Funktionen $v \in P^{1}\left(\mathcal{T}_{h}\right)$. Damit liefern (4.20) und (4.21) die Behauptung.

Mit Hilfe dieser Abschätzungen läßt sich für das upwind-stabilisierte Finite-VolumenVerfahren ein Konsistenzfehler nachweisen, der im wesentlichen dem im Abschnitt 3.1 hergeleiteten der Ordnung $O(h)$ entspricht. Im einzelnen ergibt sich, wiederum mit der abkürzenden Schreibweise $C_{\varepsilon, \eta}:=\max \left\{\frac{1}{\varepsilon}, \frac{1}{\eta}\right\}$, sofort

$$
\begin{aligned}
\left|c_{h}(u, v)-c_{h}^{u p}(u, v)\right| \leqslant & C h \max \left\{1,\|b\|_{1, \infty}\right\}\left(\frac{1}{\varepsilon}\left\|A^{\frac{1}{2}} \nabla u\right\|_{0}\left\|A^{\frac{1}{2}} \nabla v\right\|_{0}+\frac{1}{\eta}\|\sqrt{q} u\|_{0}\|\sqrt{q} v\|_{0}\right) \\
& +\|\nabla \cdot b\|_{\infty} \frac{1}{\sqrt{\varepsilon \eta}}\left\|A^{\frac{1}{2}} \nabla u\right\|_{0}\|\sqrt{q} v\|_{0} \\
\leqslant & C h \max \left\{1,\|b\|_{1, \infty}\right\}\left(C_{\varepsilon, \eta}+\frac{1}{\sqrt{\varepsilon \eta}}\right)\|u\|_{\varepsilon}\|v\|_{\varepsilon}
\end{aligned}
$$

und

$$
\begin{aligned}
\left|r_{h}(u, v)-r_{h}^{u p}(u, v)\right| & \leqslant C h\|c\|_{1, \infty}\left(\frac{1}{\sqrt{\varepsilon}}\left\|A^{\frac{1}{2}} \nabla u\right\|_{0}+\frac{1}{\sqrt{\eta}}\|\sqrt{q} u\|_{0}\right) \frac{1}{\sqrt{\eta}}\|\sqrt{q} v\|_{0} \\
& \leqslant C h\|c\|_{1, \infty} \frac{C_{\varepsilon, \eta}}{\sqrt{\eta}}\|u\|_{\varepsilon}\|\sqrt{q} v\|_{0} .
\end{aligned}
$$


Für die Differenz zwischen $a_{h}(u, v)$ und $a_{h}^{u p}(u, v)$ bedeutet dies

$$
\left|a_{h}(u, v)-a_{h}^{u p}(u, v)\right| \leqslant C h C_{K}^{u p}\|u\|_{\varepsilon}\|v\|_{\varepsilon}
$$

$\operatorname{mit} C_{K}^{u p}:=\max \left\{1,\|b\|_{1, \infty}\right\}\left(C_{\varepsilon, \eta}+\frac{1}{\sqrt{\varepsilon \eta}}\right)+\|c\|_{1, \infty} \frac{C_{\varepsilon, \eta}}{\sqrt{\eta}}$ und einer Konstante $C>0$, die weder von $h$ noch von den Daten des Problems (4.1)-(4.4) abhängt. Zieht man die Resultate aus dem Abschnitt 2.3 heran, so ergibt sich schließlich eine Schranke für den Konsistenzfehler des stabilisierten Verfahrens (4.10)-(4.11):

Lemma 4.3.2 Es seien a und $a_{h}^{u p}$ die Bilinearformen laut (4.2) bzw. (4.11), wobei $b \in$ $\left(H^{1, \infty}(\Omega)\right)^{2}$ und $c \in H^{1, \infty}(\Omega)$ vorausgesetzt sei. Dann gilt für je zwei Funktionen $u, v \in P^{1}\left(\mathcal{T}_{h}\right)$

$$
\left|a(u, v)-a_{h}^{u p}(u, v)\right| \leqslant h\left(C_{K}+C_{K}^{u p} C\right)\|u\|_{\varepsilon}\|v\|_{\varepsilon}
$$

mit einer Konstante $C>0$, die weder von $h$ noch von den Daten des Problems (4.1)-(4.4) abhängt.

Beweis. Wegen

$$
\left|a(u, v)-a_{h}^{u p}(u, v)\right| \leqslant\left|a(u, v)-a_{h}(u, v)\right|+\left|a_{h}(u, v)-a_{h}^{u p}(u, v)\right|
$$

ergibt sich die Behauptung unmittelbar aus (3.7) und (4.21).

Als Folgerung ergibt sich wie im Abschnitt 3.2 die Stetigkeit der Bilinearform $a_{h}^{u p}$ bezüglich der Norm $\|\cdot\|_{\varepsilon}$ unter Ausnutzung der Stetigkeit der Bilinearform a gemäß (3.13):

$$
\left|a_{h}^{u p}(u, v)\right| \leqslant|a(u, v)|+\left|a(u, v)-a_{h}^{u p}(u, v)\right| \leqslant\left(M_{\|\cdot\|_{\varepsilon}}+h\left(C_{K}+C_{K}^{u p} C\right)\right)\|u\|_{\varepsilon}\|v\|_{\varepsilon}
$$

$\operatorname{mit} M_{\|\cdot\|_{\varepsilon}}=1+\frac{\|b\|_{\infty}}{2 \sqrt{\varepsilon \eta}}$.

\subsection{A-priori-Fehlerabschätzung}

Abschließend übertragen wir die a-priori-Fehlerabschätzung aus Satz 3.3.3 auf das UpwindFinite-Volumen-Verfahren. Die Beschränkung an $h$ in Abhängigkeit von $\varepsilon$, die dort nötig war, kann dabei fallen gelassen werden.

Satz 4.4.1 Es sei (TR) vorausgesetzt und $u \in H^{1}(0, T ; V)$ Lösung von (4.1)-(4.4) sowie $u_{h} \in H^{1}\left(0, T ; P_{0}^{1}\left(\mathcal{T}_{h}\right)\right)$ Lösung der zugehörigen Finite-Volumen-Diskretisierung (4.10) - (4.12) zum Donald-Diagramm $\mathcal{B}_{h}^{D}$ zu $\mathcal{T}_{h}$. Bedingung (4.3) sei verschärft durch $b \in\left(H^{1, \infty}(\Omega)\right)^{2}$, $c \in H^{1, \infty}(\Omega)$. Ferner seien die Voraussetzungen aus Satz 4.2.2 und die Regularitätsforderung aus Lemma 3.3 .1 an das adjungierte Problem (3.15) erfüllt. Dann gilt fast überall in $(0, T)$ die Abschätzung

$$
\begin{aligned}
\left\|e_{h}(t)\right\|_{0}^{2}+\int_{0}^{t}\left\|e_{h}(s)\right\|_{\varepsilon}^{2} d s \leqslant & \left\|e_{h}(0)\right\|_{0}^{2}+\frac{h^{2}}{\alpha_{u p}}\left(C_{K}+C_{K}^{u p} C\right)^{2} C_{1}\left\|u_{0, h}\right\|_{0}^{2} \\
& +\left(\frac{1}{\varepsilon}+\frac{\left(C_{K}+C_{K}^{u p} C\right) C_{d}}{\eta \alpha_{u p}^{2}}\right) h^{2} C_{1} \int_{0}^{t}\|f(s)\|_{0}^{2} d s \\
& +h^{2} C_{2} \int_{0}^{t}\|u(s)\|_{2}^{2} d s+h^{2} \int_{0}^{t}\left\|u^{\prime}(s)\right\|_{0}^{2}+\left\|u_{h}^{\prime}(s)\right\|_{0}^{2} d s
\end{aligned}
$$


mit $C_{1}:=4 M_{\|\cdot\|_{\varepsilon}}^{2}, C_{2}:=2 C_{R}^{2}\left(1+M_{\|\cdot\|_{\varepsilon}}^{2}\right)$, sofern $u(t) H^{2}(\Omega)$-regulär ist für fast alle $t \in(0, T)$. Dabei ist $C_{K}+C_{K}^{u p} C$ die Konstante aus Lemma 4.3.2.

Beweis. $\quad$ Es läßt sich direkt der Beweis von Satz 3.3.3 heranziehen, wenn man die Fehlergleichung (3.4) für

$$
A_{h}(t, v):=a\left(u_{h}(t), v\right)-a_{h}^{u p}\left(u_{h}(t), v\right)
$$

aufstellt und die im genannten Beweis verwendete Abschätzung für $A_{h}(t, v)$ und $F_{h}(t, v)$ aufgrund von Lemma 4.3.2 ersetzt durch

$$
\left|A_{h}(t, v)\right|+\left|F_{h}(t, v)\right| \leqslant h\left(\left(C_{K}+C_{K}^{u p} C\right)\left\|u_{h}(t)\right\|_{\varepsilon}+\frac{\|f(t)\|_{0}}{\sqrt{\varepsilon}}\right)\|v\|_{\varepsilon} .
$$

Zur Abschätzung von $\left\|u_{h}(t)\right\|_{\varepsilon}$ durch die Daten zieht man entsprechend Lemma 4.2 .4 für $\lambda=0$, also

$$
\int_{0}^{t}\left\|u_{h}(s)\right\|_{\varepsilon}^{2} d s \leqslant \frac{1}{\alpha_{u p}}\left\|u_{0, h}\right\|_{0}^{2}+\frac{C_{d}}{\eta \alpha_{u p}^{2}} \int_{0}^{t}\|f(s)\|_{0}^{2} d s
$$

heran.

Wir haben in diesem Kapitel eine Upwind-Modifikation des in dieser Arbeit betrachteten Finite-Volumen-Verfahrens eingeführt und untersucht. Damit steht dieses Verfahren auch für konvektionsdominierte zeitabhängige Konvektions-Diffusions-Reaktions-Probleme zur Verfügung. Die Stabilität des Verfahrens zeigte sich dabei unabhängig von der Stärke $\varepsilon$ der Diffusion. Durch Satz 4.4.1 ergab sich (unter geeigneten Regularitätvorderungen) analytisch eine Fehlerordnung von $O(h)$ bezüglich der $L^{2}$-Norm. Eine Halbierung des Gitterparameters $h$ zieht hier also eine Halbierung des Fehlers nach sich.

Kritisch zu betrachten sind dabei die Koeffizienten, die reziprok von einer Potenz von $\varepsilon$ abhängen. So treten in der Abschätzung (4.23) die Faktoren $\frac{1}{\varepsilon}, \frac{1}{\sqrt{\varepsilon}}$ und $\max \left\{\frac{1}{\varepsilon}, \frac{1}{\eta}\right\}$ auf. Prinzipiell dieselben Probleme treten jedoch auch im Fall von SUPG-stabilisierten Finite-ElementVerfahren auf.

Mit diesem Kapitel schließen sich die Voraussetzung für die Formulierung der Finite-VolumenDiskretisierung im Zusammenhang mit einem Gebietszerlegungsverfahren, wie es im zweiten Teil dieser Arbeit entwickelt wird. 


\section{Teil II}

\section{Gebietszerlegungsverfahren}





\section{Kapitel 5}

\section{Gebietszerlegungsverfahren für parabolische Probleme}

In diesem Kapitel entwickeln wir ein Gebietszerlegungsverfahren bezüglich nichtüberlappender Gebiete für zeitabhängige Konvektions-Diffusions-Reaktions-Probleme. Zunächst leiten wir eine Zweigebietsformulierung für Evolutionsprobleme her, die gleichwertig ist zu der ursprünglichen Problemstellung aus Kapitel 1.

Diese Zweigebietsformulierung ist dann Ausgangspunkt für das im Rest des Kapitels konstruierte iterative Verfahren. Es handelt sich dabei um ein Verfahren mit einer Kopplung über die Austauschränder vom Dirichlet-Robin-Typ und mit zeitkontinuierlichen Problemen in jedem Teilgebiet und jedem Iterationsschritt.

\subsection{Zweigebietsformulierung}

In diesem Abschnitt geben wir eine Zweigebietsformulierung für die im Abschnitt 1.1 vorgestellten Evolutionsprobleme 1. Ordnung

Finde $u \in H^{1}(0, T ; V)$ mit

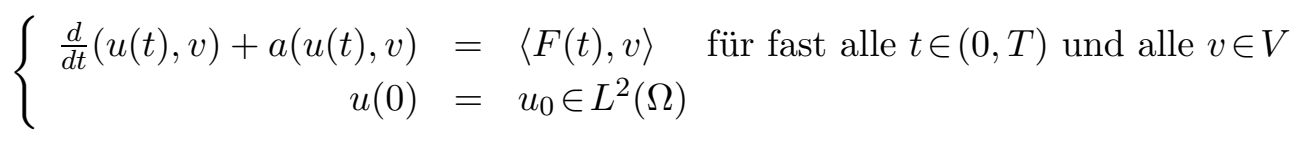

an. Dazu sei $V=H_{0}^{1}(\Omega)$ und $\left\{\Omega_{1}, \Omega_{2}\right\}$ eine Zerlegung von $\Omega$ in zwei Lipschitz-Gebiete, es gelte also

$$
\overline{\Omega_{1}} \cup \overline{\Omega_{2}}=\bar{\Omega} \quad \text { und } \quad \Omega_{1} \cap \Omega_{2}=\emptyset .
$$

Die Teilgebiete besitzen einen gemeinsamen Rand $\Gamma:=\overline{\Omega_{1}} \cap \overline{\Omega_{2}}$, der künstlicher Rand oder im Zusammenhang mit iterativen Gebietszerlegungsverfahren Austauschrand genannt wird. Abbildung 5.1 zeigt schematisch zwei mögliche Zerlegungen. 

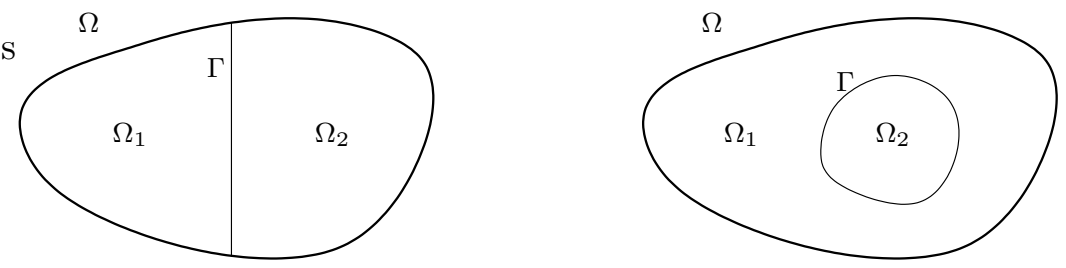

Abbildung 5.1: Zwei Zerlegungen des Gebiets $\Omega$ in Lipschitz-Gebiete $\Omega_{1}, \Omega_{2}$

Bezogen auf diese Zerlegung führen wir die Räume

$$
V_{i}:=\left.V\right|_{\Omega_{i}}=\left\{\left.v\right|_{\Omega_{i}} \mid v \in V\right\}, \quad V_{i, 0}:=H_{0}^{1}\left(\Omega_{i}\right) \quad \text { und } \quad W_{i}:=\operatorname{tr}_{\Gamma, i}\left[V_{i}\right]
$$

ein. Dabei bezeichne $\operatorname{tr}_{\Gamma, i}$ den Spuroperator auf $H^{1}\left(\Omega_{i}\right)$ bezüglich (siehe Satz A.2.3 und anschließende Bemerkung). Das folgende Lemma erlaubt die Identifizierung von Funktionen $v \in V$ mit Paaren von Funktionen über den Teilgebieten, die auf $\Gamma$ identische Randwerte besitzen.

Lemma 5.1.1 Es sei $\left\{\Omega_{1}, \Omega_{2}\right\}$ eine Zerlegung von $\Omega$ in zwei Lipschitz-Gebiete. Mit den oben eingeführten Bezeichnungen gilt dann

$$
V=\left\{\left(u_{1}, u_{2}\right) \in V_{1} \times V_{2}\left|u_{1}\right|_{\Gamma}=\left.u_{2}\right|_{\Gamma}\right\}
$$

Beweis. $\quad$ Nutze partielle Integration sowie $u_{i}=\left.u\right|_{\Omega_{i}} \in H^{1}\left(\Omega_{i}\right) \operatorname{mit} D^{\alpha} u_{i}=\left.\left(D^{\alpha} u\right)\right|_{\Omega_{i}}$ für $u \in V$. Die Gleichung $\left.u_{1}\right|_{\Gamma}=\left.u_{2}\right|_{\Gamma}$ ist als Gleichung in $H^{\frac{1}{2}}(\Gamma)$ anzusehen.

Es folgt unmittelbar $W_{1}=W_{2}=: W$. Falls $\Gamma$ und $\partial \Omega$ disjunkt sind, ist $W=H^{\frac{1}{2}}(\Gamma)$, sonst gilt $W=H_{00}^{\frac{1}{2}}(\Gamma)$. Vereinfachend schreiben wir $\|\cdot\|_{W}$ für die jeweils zugehörige Norm (siehe Anhang A.2).

Es seien $a_{1}, a_{2}$ Bilinearformen auf $H^{1}\left(\Omega_{1}\right) \times H^{1}\left(\Omega_{1}\right)$ bzw. $H^{1}\left(\Omega_{2}\right) \times H^{1}\left(\Omega_{2}\right)$ mit

$$
a(u, v)=a_{1}\left(\left.u\right|_{\Omega_{1}},\left.v\right|_{\Omega_{1}}\right)+a_{2}\left(\left.u\right|_{\Omega_{2}},\left.v\right|_{\Omega_{2}}\right) \quad \text { für alle } u, v \in V
$$

sowie $F_{i} \in L^{2}\left(0, T ; V_{i}^{*}\right), i=1,2$, mit

$$
\langle F(t), v\rangle=\left\langle F_{1}(t),\left.v\right|_{\Omega_{1}}\right\rangle+\left\langle F_{2}(t),\left.v\right|_{\Omega_{2}}\right\rangle \quad \text { für alle } v \in V \text {. }
$$

Die Zweigebietsformulierung zum Evolutionsproblem (5.1) bezüglich der Zerlegung $\left\{\Omega_{1}, \Omega_{2}\right\}$ von $\Omega$ sowie der Bilinearformen $a_{i}$ und Linearformen $F_{i}, i=1,2$, lautet: 
Finde $\left(u_{1}, u_{2}\right) \in H^{1}\left(0, T ; V_{1}\right) \times H^{1}\left(0, T ; V_{2}\right)$ mit

$$
\left\{\begin{array}{c}
\frac{d}{d t}\left(u_{i}(t), v\right)_{\Omega_{i}}+a_{i}\left(u_{i}(t), v\right)=\left\langle F_{i}(t), v\right\rangle \\
\quad \text { für fast alle } t \in(0, T) \text { und alle } v \in V_{i, 0}, \quad i=1,2 \\
\left.u_{1}(t)\right|_{\Gamma}=\left.u_{2}(t)\right|_{\Gamma} \quad \text { für fast alle } t \in(0, T) \\
\sum_{i \in \underline{2}} \frac{d}{d t}\left(u_{i}(t), r_{i} w\right)_{\Omega_{i}}+a_{i}\left(u_{i}(t), r_{i} w\right)=\sum_{i \in \underline{2}}\left\langle F_{i}(t), r_{i} w\right\rangle \\
\text { für fast alle } t \in(0, T) \text { und alle } w \in W \\
u_{i}(0)=\left.u_{0}\right|_{\Omega_{i}} \in L^{2}\left(\Omega_{i}\right), \quad i=1,2
\end{array}\right.
$$

wobei $r_{i}$ für eine beliebige Rechtsinverse $\mathrm{zu} \mathrm{tr}_{\Gamma, i}$ steht (eine solche existiert nach A.2.3).

Die beiden lokalen Probleme, aus denen sich (5.2) zusammensetzt, werden also durch die Austauschrandbedingungen $(5.2)_{1}$ und $(5.2)_{2}$ miteinander verknüpft. Die beiden Formulierungen (5.1) und (5.2) sind im folgenden Sinne äquivalent.

Lemma 5.1.2 Ist $u \in H^{1}(0, T ; V)$ eine Lösung von (5.1), so löst $\left(u_{1}, u_{2}\right)$ mit $u_{i}:=\left.u\right|_{\Omega_{i}}$ das Problem (5.2). Ist umgekehrt $\left(u_{1}, u_{2}\right)$ eine Lösung von (5.2), so ist die zusammengesetzte Funktion u mit

$$
u(t):= \begin{cases}u_{1}(t) & \text { in } \Omega_{1} \\ u_{2}(t) & \text { in } \Omega_{2}\end{cases}
$$

eine Lösung von (5.1).

Beweis. $\quad$ Es sei $u$ eine Lösung von (5.1) und $v_{i} \in V_{i, 0}$. Dann ist die triviale Fortsetzung

$$
v^{\circ}:= \begin{cases}v_{i} & \text { in } \Omega_{i} \\ 0 & \text { in } \Omega \backslash \Omega_{i}\end{cases}
$$

in $V$, nach Voraussetzung gilt daher

$$
\left\langle F_{i}(t), v_{i}\right\rangle-\frac{d}{d t}\left(u_{i}(t), v_{i}\right)_{\Omega_{i}}=\left\langle F(t), v^{\circ}\right\rangle-\frac{d}{d t}\left(u_{i}(t), v^{\circ}\right)_{\Omega_{i}}=a\left(u(t), v^{\circ}\right)=a_{i}\left(u_{i}(t), v_{i}\right) .
$$

Gleichung (5.2) 2 gilt wegen Lemma 5.1.1.

Außerdem sei für jedes $w \in W$

$$
r w:= \begin{cases}r_{1} w & \text { in } \Omega_{1} \\ r_{2} w & \text { in } \Omega_{2} .\end{cases}
$$


Wiederum mit Lemma 5.1 .1 folgt $r w \in V$, da $\left.r_{1} w\right|_{\Gamma}=\left.r_{2} w\right|_{\Gamma}$. Somit ist

$$
\begin{aligned}
\sum_{i \in \underline{2}} \frac{d}{d t}\left(u_{i}(t), r_{i} w\right)_{\Omega_{i}}+a_{i}\left(u_{i}(t), r_{i} w\right) & =\frac{d}{d t}(u(t), r w)_{\Omega}+a(u(t), r w) \\
& =\langle F(t), r w\rangle \\
& =\sum_{i \in \underline{2}}\left\langle F_{i}(t), r_{i} w\right\rangle .
\end{aligned}
$$

Umgekehrt folgt $u(t) \in V$ (mit $u$ laut (5.3)) fast überall in $(0, T)$ wegen $(5.2)_{2}$ und Lemma 5.1.1, falls $\left(u_{1}, u_{2}\right)$ das Zweigebietsproblem löst. Für jedes $v \in V$ ist

$$
w:=\operatorname{tr}_{\Gamma, 1}\left(\left.v\right|_{\Omega_{1}}\right)=\operatorname{tr}_{\Gamma, 2}\left(\left.v\right|_{\Omega_{2}}\right) \in W \text { und }\left.v\right|_{\Omega_{i}}-r_{i} w \in V_{i, 0}, \quad i=1,2
$$

Daher folgt mit $(5.2)_{3}$ und $(5.2)_{1}$

$$
\begin{aligned}
\frac{d}{d t}(u(t), v)_{\Omega}+a(u(t), v)= & \sum_{i \in \underline{2}} \frac{d}{d t}\left(u_{i}(t),\left.v\right|_{\Omega_{i}}\right)_{\Omega_{i}}+a_{i}\left(u_{i}(t),\left.v\right|_{\Omega_{i}}\right) \\
= & \sum_{i \in \underline{2}} \frac{d}{d t}\left(u_{i}(t),\left.v\right|_{\Omega_{i}}-r_{i} w\right)_{\Omega_{i}}+a_{i}\left(u(t),\left.v\right|_{\Omega_{i}}-r_{i} w\right) \\
& +\sum_{i \in \underline{2}} \frac{d}{d t}\left(u_{i}(t), r_{i} w\right)_{\Omega_{i}}+a_{i}\left(u(t), r_{i} w\right) \\
= & \sum_{i \in \underline{2}}\left\langle F_{i}(t),\left.v\right|_{\Omega_{i}}-r_{i} w\right\rangle+\sum_{i \in \underline{2}}\left\langle F_{i}(t), r_{i} w\right\rangle \\
= & \left\langle F_{1}(t),\left.v\right|_{\Omega_{1}}\right\rangle+\left\langle F_{2}(t),\left.v\right|_{\Omega_{2}}\right\rangle=\langle F(t), v\rangle
\end{aligned}
$$

für fast alle $t \in(0, T)$ und alle $v \in V$.

Offensichtlich kann die Gleichung $(5.2)_{3}$ wegen der Übereinstimmung $(5.2)_{2}$ der Randwerte ersetzt werden durch

$$
\sum_{i \in \underline{2}}\left[\frac{d}{d t}\left(u_{i}(t), r_{i} w\right)_{\Omega_{i}}+a_{i}\left(u_{i}(t), r_{i} w\right)\right]+\left\langle\left.\gamma u_{2}(t)\right|_{\Gamma}, w\right\rangle_{W}=\left\langle\left.\gamma u_{1}(t)\right|_{\Gamma}, w\right\rangle_{W}+\sum_{i \in \underline{2}}\left\langle F_{i}(t), r_{i} w\right\rangle
$$

für fast alle $t \in(0, T)$ und alle $w \in W$

für jedes Skalarprodukt $\langle\cdot, \cdot\rangle_{W}$ auf $W$ und jede Funktion $\gamma \in L^{\infty}(\Gamma)$. Dies entspricht dem Übergang der Bilinearformen von $a_{i}$ zu $\tilde{a}_{i}, i=1,2$, mit

$$
\tilde{a}_{1}(u, v):=a_{1}(u, v)-\left\langle\left.\gamma u\right|_{\Gamma},\left.v\right|_{\Gamma}\right\rangle_{W}, \quad \tilde{a}_{2}(u, v):=a_{2}(u, v)+\left\langle\left.\gamma u\right|_{\Gamma},\left.v\right|_{\Gamma}\right\rangle_{W} .
$$

Diese Form gewinnt Bedeutung, wenn wir im nächsten Abschnitt iterative Gebietszerlegungsmethoden betrachten und dabei auf einem Teilgebiet $\Omega_{i}$ ein Anfangs-Randwertproblem mit Robin-Bedingung formulieren. 
Bemerkung 5.1.3 Es sei $n_{i}$ die äußere Normale an $\Omega_{i}, n_{\Gamma, i}$ ihre Restriktion auf $\Gamma$ und $\operatorname{ntr}_{\Gamma, i}$ der Normalen-Spuroperator auf $H^{1}\left(\Omega_{i}\right)$ bezüglich $\Gamma$ (siehe A.2.4). Im Falle eines KonvektionsDiffusions-Reaktions-Problems, also

$$
a_{i}(u, v):=\int_{\Omega_{i}} \nabla v \cdot A \nabla u+v \nabla \cdot(u b)+c u v d x
$$

bezeichnet man

$$
\frac{\partial u_{i}(t)}{\partial n_{L}}:=A \nabla u_{i}(t) \cdot n_{i}=\operatorname{ntr}_{\Gamma, i}\left(A \nabla u_{i}(t)\right)
$$

auch als Konormalen-Ableitung von $u_{i}(t)$. Die Bedingung (5.2) $)_{3}$ impliziert die „physikalischere" Flußbedingung

$$
\frac{\partial u_{1}(t)}{\partial n_{L}}=-\frac{\partial u_{2}(t)}{\partial n_{L}}
$$

auf dem Austauschrand $\Gamma$, denn für die Bilinearformen $a_{1}, a_{2}$ gilt nach der Greenschen Formel (siehe A.2.5) für $u \in H_{0}^{1}(\Omega ; A \nabla):=\{u \in V \mid A \nabla u \in H(\operatorname{div} ; \Omega)\}$

$$
a_{i}(u, v)=\int_{\Omega_{i}} v L u d x+\left\langle\operatorname{ntr}_{\partial \Omega_{i}}(A \nabla u), \operatorname{tr}_{\partial \Omega_{i}} v\right\rangle \quad u, v \in V_{i}
$$

mit $L u=-\nabla \cdot(A \nabla u-b u)+c u$ wie in (1.5). Nach (5.2) 1 gilt mit $\left\langle F_{i}(t), v\right\rangle=(f(t), v)_{\Omega_{i}}$

$$
(f(t), v)_{\Omega_{i}}=\frac{d}{d t}\left(u_{i}(t), v\right)_{\Omega_{i}}+a_{i}\left(u_{i}(t), v\right)=\frac{d}{d t}\left(u_{i}(t), v\right)_{\Omega_{i}}+\int_{\Omega_{i}} v L u_{i}(t) d x \quad v \in C_{0}^{\infty}\left(\Omega_{i}\right),
$$

also $u_{i}^{\prime}(t)+L u_{i}(t)=f(t)$ in $L^{2}\left(\Omega_{i}\right)$ für fast alle $t \in(0, T)$. Mit $(5.2)_{3}$ folgt weiter

$$
\begin{aligned}
& 0= \sum_{i \in \underline{2}}\left(\frac{d}{d t}\left(u_{i}(t), r_{i} w\right)_{\Omega_{i}}+a_{i}\left(u_{i}(t), r_{i} w\right)-\left(f(t), r_{i} w\right)_{\Omega_{i}}\right) \\
&=\sum_{i \in \underline{2}}\left(\frac{d}{d t}\left(u_{i}(t), r_{i} w\right)_{\Omega_{i}}+\int_{\Omega_{i}} r_{i} w L u_{i}(t) d x\right. \\
&\left.\quad+\left\langle\operatorname{ntr}_{\partial \Omega_{i}}\left(A \nabla u_{i}(t)\right), \operatorname{tr}_{\partial \Omega_{i}} r_{i} w\right\rangle-\left(f(t), r_{i} w\right)_{\Omega_{i}}\right) \\
&=\sum_{i \in \underline{2}}\left\langle\operatorname{ntr}_{\Gamma, i}\left(A \nabla u_{i}(t)\right), w\right\rangle
\end{aligned}
$$

für jedes $w \in W$. Dies bedeutet gerade die Gültigkeit von (5.4) in $W^{*}$ für fast alle $t \in(0, T)$. $\diamond$

\subsection{Ein Dirichlet-Robin-Algorithmus}

Der im vorigen Abschnitt hergeleitete Zusammenhang zwischen globalem Problem und Zweigebietsformulierung dient uns als Vorlage, um in diesem Abschnitt ein iteratives 
Gebietszerlegungsverfahren zu konstruieren für zeitabhängige Konvektions-DiffusionsReaktions-Probleme der Form:

Finde $u \in H^{1}(0, T ; V)$ mit

$$
\left\{\begin{aligned}
\frac{d}{d t}(u(t), v)+a(u(t), v) & =(f(t), v) \quad \text { für fast alle } t \in(0, T) \text { und alle } v \in V \\
u(0) & =u_{0} \in L^{2}(\Omega)
\end{aligned}\right.
$$

mit

$$
a(u, v):=\int_{\Omega} \varepsilon \nabla v \cdot \nabla u+v \nabla \cdot(u b)+c u v d x
$$

und $V=H_{0}^{1}(\Omega)$.

Wir betrachten also speziell Probleme mit der Diffusionsmatrix $A=\left(\varepsilon \delta_{i j}\right)_{i, j \in \underline{d}}$. Außer den Anforderungen

$$
\varepsilon>0, \quad b_{j}, \nabla \cdot b, c \in L^{\infty}(\Omega), \quad f \in L^{2}\left(Q_{T}\right)
$$

an die Daten setzen wir

$$
\eta:=\underset{\Omega}{\operatorname{essinf}} q>0 \text { mit } q:=\frac{1}{2} \nabla \cdot b+c
$$

voraus, um die $V$-Koerzivität der Bilinearform $a$ zu gewährleisten. Nach (1.7) erhält man

$$
a(u, v)=\int_{\Omega} \varepsilon \nabla v \cdot \nabla u+\left(\frac{1}{2} \nabla \cdot b+c\right) u v d x+\frac{1}{2} \int_{\Omega} v b \cdot \nabla u-u b \cdot \nabla v d x,
$$

wodurch die Bilinearform $a$ in einen symmetrischen und einen schiefsymmetrischen Anteil zerlegt wird.

Nach Lemma 5.1.2 ist (5.5) gleichwertig zu der Zweigebietsformulierung

Finde $\left(u_{1}, u_{2}\right) \in H^{1}\left(0, T ; V_{1}\right) \times H^{1}\left(0, T ; V_{2}\right)$ mit

$$
\left\{\begin{array}{c}
\frac{d}{d t}\left(u_{i}(t), v\right)_{\Omega_{i}}+a_{i}\left(u_{i}(t), v\right)=(f(t), v)_{\Omega_{i}} \\
\quad \text { für fast alle } t \in(0, T) \text { und alle } v \in V_{i, 0}, \quad i=1,2 \\
\left.u_{1}(t)\right|_{\Gamma}=\left.u_{2}(t)\right|_{\Gamma} \quad \text { für fast alle } t \in(0, T) \\
\sum_{i \in \underline{2}}\left[\frac{d}{d t}\left(u_{i}(t), r_{i} w\right)_{\Omega_{i}}+a_{i}\left(u_{i}(t), r_{i} w\right)\right]+\left\langle\left.\gamma u_{2}(t)\right|_{\Gamma}, w\right\rangle_{W}=\sum_{i \in \underline{2}}\left(f(t), r_{i} w\right)_{\Omega_{i}} \\
+\left\langle\left.\gamma u_{1}(t)\right|_{\Gamma}, w\right\rangle_{W} \quad \text { für fast alle } t \in(0, T) \text { und alle } w \in W \\
u_{i}(0)=\left.u_{0}\right|_{\Omega_{i}} \in L^{2}\left(\Omega_{i}\right), \quad i=1,2,
\end{array}\right.
$$


wobei $\gamma$ eine beliebige Funktion aus $L^{\infty}(\Gamma)$ und $\langle\cdot, \cdot\rangle_{W}$ das Skalarprodukt auf $W$ ist, $r_{i}$ wiederum eine beliebige Rechtsinverse $\mathrm{zu} \operatorname{tr}_{\Gamma, i}$ bezeichnet und die Bilinearformen $a_{1}, a_{2}$ definiert sind durch

$$
a_{i}(u, v):=\int_{\Omega_{i}} \varepsilon \nabla v \cdot \nabla u+\left(\frac{1}{2} \nabla \cdot b+c\right) u v d x+\frac{1}{2} \int_{\Omega_{i}} v b \cdot \nabla u-u b \cdot \nabla v d x, \quad u, v \in V_{i} .
$$

Beide Bilinearformen sind nach Lemma 1.1 .3 stetig und koerziv auf $V_{1} \times V_{1}$ bzw. $V_{2} \times V_{2}$ :

$$
a_{i}(u, v) \leqslant M_{i}\|u\|_{1, \Omega_{i}}\|v\|_{1, \Omega_{i}}, \quad a_{i}(v, v) \geqslant \alpha_{i}\|v\|_{1, \Omega_{i}} \quad \text { für alle } u, v \in V_{i}
$$

mit den Stetigkeits- und Koerzivitätskonstanten

$$
M_{i}=M_{\Omega_{i}}=\varepsilon+\|q\|_{\infty, \Omega_{i}}+\|b\|_{\infty, \Omega_{i}} \text { und } \alpha_{i}=\alpha_{\Omega_{i}}=\min \{\varepsilon, \eta\}, \quad i=1,2 .
$$

Der klassische Weg, parabolische Anfangs-Randwertprobleme mit Hilfe von Gebietszerlegungsmethoden zu lösen, besteht darin, zunächst in der Zeit implizit zu diskretisieren, um dann in jedem Zeitschritt einen Gebietszerlegungsalgorithmus auf das entstandene stationäre (elliptische) Problem anzuwenden (siehe etwa [13, 14, 18, 46]).

Im Gegensatz dazu soll hier ein iteratives Schema konstruiert werden, dem das (zeitkontinuierliche) Problem (5.10) als Ausgangspunkt dient, so daß eine Folge von parabolischen Problemen, also Anfangsrandwertproblemen auf jedem Teilgebiet ensteht. Damit entsteht ein Gebietszerlegungsverfahren vom Waveform-Relaxation-Typ.

Wir greifen dabei Techniken aus der Arbeit [2], in der allerdings ausschließlich stationäre Probleme betrachtet werden, auf und übertragen diese auf zeitabhängige Probleme. Dort werden iterative Verfahren sowohl mit Dirichlet-Randbedingungen auf einem und RobinRandbedingungen auf dem anderen Teilgebiet (Dirichlet-Robin-Algorithmus) als auch solche mit Robin-Randbedingungen auf beiden Teilgebieten (Robin-Robin-Algorithmus) für Konvektions-Diffusions-Reaktions-Probleme betrachtet.

Durch Vorgabe nur einer Randbedingung der Art (5.10) 2 oder (5.10) 3 auf einem der Teilgebiete ist die Lösung auf diesem Teilgebiet bereits eindeutig bestimmt (vergl. Ende des Abschnitts). Die Erfüllung einer zweiten, von der ersten verschiedenen Randbedingung ist also gleichbedeutend damit, die zugehörige Lösung bereits zu kennen. Beim Übergang zum iterativen Schema wird daher pro Teilgebiet nur jeweils eine der Randbedingungen herangezogen.

Die Stetigkeitsforderung (5.10) 2 wird ersetzt durch eine Relaxationsgleichung für die Randwerte von $u_{1}$ und $u_{2}$, um in jedem Iterationsschritt eine Dirichlet-Bedingung auf dem einen Teilgebiet vorzugeben. Zur Formulierung der entsprechenden Teilprobleme mit zeitabhängigen Dirichlet-Randwerten nutzen wir den Spurraum

$$
Z:=L^{2}(0, T ; W) \cap H^{\frac{1}{4}}\left(0, T ; L^{2}(\Gamma)\right),
$$

in den die nötige Regularität in der Zeit einfließt (siehe A.3).

Die Gleichung (5.10) ${ }_{3}$ dient hingegen der Formulierung von Robin-Randbedingungen auf dem komplementären Teilgebiet. Die Einführung des zusätzlichen Parameters $\gamma$ wie in (5.10) stellt die Konvergenz des Verfahrens prinzipiell auch im konvektionsdominanten Fall sicher. 
In Gänze lautet der Dirichlet-Robin-Algorithmus mit Parameter $\gamma \in L^{\infty}(\Gamma)$ :

$\mathrm{Zu}$ einem Initialwert $g^{0} \in Z$ finde eine Folge von Funktionen $\left(u_{1}^{k}, u_{2}^{k}\right) \in H^{1}\left(0, T ; V_{1}\right) \times$ $H^{1}\left(0, T ; V_{2}\right)$ mit

$$
\begin{aligned}
& \left\{\begin{array}{c}
\frac{d}{d t}\left(u_{1}^{k}(t), v\right)_{\Omega_{1}}+a_{1}\left(u_{1}^{k}(t), v\right)=(f(t), v)_{\Omega_{1}} \\
\quad \text { für fast alle } t \in(0, T) \text { und alle } v \in V_{1,0} \\
\begin{array}{l}
\left.u_{1}^{k}(t)\right|_{\Gamma}=g^{k-1}(t) \quad \text { für fast alle } t \in(0, T) \\
u_{1}^{k}(0)=\left.u_{0}\right|_{\Omega_{1}} \in L^{2}\left(\Omega_{1}\right),
\end{array} \\
\left\{\begin{array}{c}
\frac{d}{d t}\left(u_{2}^{k}(t), v\right)_{\Omega_{2}}+a_{2}\left(u_{2}^{k}(t), v\right)=(f(t), v)_{\Omega_{2}} \\
\text { für fast alle } t \in(0, T) \text { und alle } v \in V_{2,0} \\
\frac{d}{d t}\left(u_{2}^{k}(t), r_{2} w\right)_{\Omega_{2}}+a_{2}\left(u_{2}^{k}(t), r_{2} w\right)+\left\langle\left.\gamma u_{2}^{k}(t)\right|_{\Gamma}, w\right\rangle_{W}=\left(f(t), r_{2} w\right)_{\Omega_{2}} \\
+\left(f(t), r_{1} w\right)_{\Omega_{1}}-\frac{d}{d t}\left(u_{1}^{k}(t), r_{1} w\right)_{\Omega_{1}}-a_{1}\left(u_{1}^{k}(t), r_{1} w\right)+\left\langle\left.\gamma u_{1}^{k}(t)\right|_{\Gamma}, w\right\rangle_{W} \\
\text { für fast alle } t \in(0, T) \text { und alle } w \in W
\end{array}\right. \\
u_{2}^{k}(0)=\left.u_{0}\right|_{\Omega_{2}} \in L^{2}\left(\Omega_{2}\right),
\end{array}\right.
\end{aligned}
$$

für jedes $k \geqslant 1$. Dabei ist

$$
g^{k}:=\left.\sigma u_{2}^{k}\right|_{\Gamma}+(1-\sigma) g^{k-1}
$$

mit einem Relaxationsparameter $\sigma \in(0,1]$.

Eine solche Folge $\left(\left(u_{1}^{k}, u_{2}^{k}\right)\right)_{k \in \mathbb{N}}$ nennen wir Lösung des Iterationsschemas (5.13) - (5.15). Ziel ist es, Werte der Parameter $\sigma$ und $\gamma$ zu bestimmen, so daß die Konvergenz der Folge gegen das Lösungspaar $\left(u_{1}, u_{2}\right)$ zum Problem (5.10) gewährleistet ist. In diesem Fall werden die ursprünglichen Randwerte dann im Grenzwert erfüllt.

Gleichung (5.15) ist die erwähnte Relaxationsgleichung, die in jedem Iterationsschritt $k$ die beiden Randwerte $\left.u_{2}^{k-1}\right|_{\Gamma}$ und $\left.u_{1}^{k-1}\right|_{\Gamma}$ aus dem vorausgegangenen Iterationsschritt konvex mischt.

Bemerkung 5.2.1 Als dynamische Variante der Relaxationsparameterwahl kann etwas allgemeiner auch zu jedem Iterationsschritt separat ein Relaxationsparameter $\sigma^{k}$ bestimmt werden.

Bemerkung 5.2.2 Ist $b \in\left(H^{1, \infty}(\Omega)\right)^{d}$, so gilt

$$
a_{i}(u, v)+\frac{1}{2} \int_{\Gamma} u v b \cdot n_{i} d s=\int_{\Omega_{i}} \varepsilon \nabla v \cdot \nabla u+v \nabla \cdot(u b)+c u v d x, \quad u, v \in V_{i},
$$

wobei das Randintegral als Integral über die Randwerte von $u, v, b$ zu betrachten ist und $n_{i}$ 
die äußere Normale an $\Omega_{i}$ bezeichnet. Nach Bemerkung 5.1.3 erhält man für (5.13) und (5.14) dann die Darstellung

$$
\begin{aligned}
& \left\{\begin{array}{l}
\left(u_{1}^{k}\right)^{\prime}(t)+L u_{1}^{k}(t)=f(t) \quad \text { in } \Omega_{1} \\
u_{1}^{k}(t)=0 \quad \text { auf } \partial \Omega_{1} \cap \partial \Omega \\
u_{1}^{k}(t)=g^{k-1}(t) \quad \text { auf } \Gamma
\end{array}\right. \\
& \left\{\begin{array}{l}
\left(u_{2}^{k}\right)^{\prime}(t)+L u_{2}^{k}(t)=f(t) \quad \text { in } \Omega_{2} \\
u_{2}^{k}(t)=0 \quad \text { auf } \partial \Omega_{2} \cap \partial \Omega \\
\varepsilon \frac{\partial u_{2}^{k}(t)}{\partial n}-\left(\frac{1}{2} b \cdot n-\gamma\right) u_{2}^{k}(t)=\varepsilon \frac{\partial u_{1}^{k}(t)}{\partial n}-\left(\frac{1}{2} b \cdot n-\gamma\right) u_{1}^{k}(t) \quad \text { auf } \Gamma,
\end{array}\right.
\end{aligned}
$$

$n=n_{1}$, unter Verwendung des Differentialoperators

$$
L u:=-\varepsilon \Delta u+\nabla \cdot(u b)+c u
$$

und jeweils ergänzt durch die Anfangsbedingung (5.13) $)_{3}$ bzw. (5.14) 3 . Zudem bezeichnet $\frac{\partial v(t)}{\partial n}$ die verallgemeinerte Normalenableitung $\operatorname{ntr}_{\Gamma, i} \nabla v(t), v \in v_{i}$ (vergl. Bemerkung 5.1.3).

Um die Wohlgestelltheit des Dirichlet-Robin-Algorithmus (5.13) - (5.15) zu zeigen, führen wir mit dem nächsten Lemma einen Fortsetzungsoperators für Randfunktionen aus $Z$ ein. Auch im weiteren Verlauf wird dieser Fortsetzungsoperator noch häufig zum Einsatz kommen. Für Funktionen über den Teilgebieten, die auf dem äußeren Rand $\partial \Omega$ verschwinden, verwenden wir die Bezeichnung

$$
V_{i, \partial \Omega}^{0}:=\left\{v \in H^{1}\left(\Omega_{i}\right)|v|_{\partial \Omega \cap \partial \Omega_{i}}=0\right\}, \quad i=1,2,
$$

wobei $V_{i, \partial \Omega}^{0}=H^{1}\left(\Omega_{i}\right)$, falls $\partial \Omega \cap \partial \Omega_{i}$ eine Menge vom Maß Null bildet.

Lemma 5.2.3 $Z u$ jedem $w \in Z$ existiert eine eindeutig bestimmte Funktion $E_{i} w \in$ $H^{1}\left(0, T ; V_{i, \partial \Omega}^{0}\right), i \in \underline{2}$, die das Dirichlet-Anfangsrandwertproblem

$$
\left\{\begin{array}{l}
\frac{d}{d t}\left(E_{i} w(t), v\right)_{\Omega_{i}}+a_{i}\left(E_{i} w(t), v\right)=0 \quad \text { für fast alle } t \in(0, T) \text { und alle } v \in V_{i, 0} \\
\left.E_{i} w(t)\right|_{\Gamma}=w(t) \quad \text { für fast alle } t \in(0, T) \\
E_{i} w(0)=0
\end{array}\right.
$$

erfüllt. Der dadurch definierte Operators $E_{i}: Z \mapsto H^{1}\left(0, T ; V_{i, \partial \Omega}^{0}\right)$ ist linear und erfüllt

$$
\left\|E_{i} w\right\|_{H^{1}\left(0, T ; V_{i, \partial \Omega}^{0}\right)} \leqslant C_{E_{i}}\|w\|_{Z}
$$

mit einer von $\varepsilon$ abhängigen Konstante $C_{E_{i}}=C_{E_{i}}(\varepsilon)$. Umgekehrt gibt es eine Konstante $C_{\Omega_{i}}$, so daß jede Funktionen $v \in H^{1}\left(0, T ; V_{i, \partial \Omega}^{0}\right)$ der Spurungleichung

$$
\left\|\left.v\right|_{\Gamma}\right\|_{Z} \leqslant C_{\Omega_{i}}\|v\|_{H^{1}\left(0, T ; V_{i, \partial \Omega}^{0}\right)}
$$

genügt mit $\left.v\right|_{\Gamma}(t):=\left.v(t)\right|_{\Gamma}$, insbesondere gilt $\left.v\right|_{\Gamma} \in Z$. 
Beweis. $\quad$ Vergleiche [45], Seite 83-84.

Als erste Konsequenz aus der Existenz der Fortsetzung $E_{i} w$ für jede Randfunktion $w \in Z$ und mit Lemma 5.1.1 folgt $V_{i, \partial \Omega}^{0} \subseteq V_{i}$, somit die Gleichheit $V_{i}=V_{i, \partial \Omega}^{0}$. Abschließend beweisen wir mit Hilfe von Lemma 5.16 den folgenden Satz.

Satz 5.2.4 In jedem Iterationsschritt $k \in \mathbb{N}$ besitzen beide Teilprobleme (5.13) und (5.14) eine eindeutige Lösung in $H^{1}\left(0, T ; V_{1}\right)$ bzw. $H^{1}\left(0, T ; V_{2}\right)$, falls $\gamma \geqslant \gamma_{0}>0$.

Beweis. Die Bilinearform $a_{1}$ ist laut (5.12) $V_{1}$-koerziv und stetig. Zu $g^{k-1} \in Z, k \in \mathbb{N}$, sei $u_{1}^{*}:=E_{1} g^{k-1}$ die Fortsetzung auf $\Omega_{1}$ gemäß Lemma 5.2.3. Nach Satz 1.1.1 erfüllt ein eindeutig bestimmtes $u_{1}^{\diamond} \in H^{1}\left(0, T ; V_{1,0}\right)$ das „homogenisierte“ Problem

$$
\left\{\begin{array}{l}
\frac{d}{d t}\left(u_{1}^{\diamond}(t), v\right)_{\Omega_{1}}+a_{1}\left(u_{1}^{\diamond}(t), v\right)=(f(t), v)_{\Omega_{1}}-\frac{d}{d t}\left(u_{1}^{*}(t), v\right)_{\Omega_{1}}-a_{1}\left(u_{1}^{*}(t), v\right) \\
\quad \text { für fast alle } t \in(0, T) \text { und alle } v \in V_{1,0} \\
u_{1}^{\diamond}(0)=\left.u_{0}\right|_{\Omega_{1}} .
\end{array}\right.
$$

Dann ist $u_{1}^{k}:=u_{1}^{\diamond}+u_{1}^{*}$ Lösung des Dirichlet-Schritts (5.13). Für den Nachweis der Eindeutigkeit beachte man, daß für jede weitere Lösung $w_{1}^{k}$ von (5.13) im Iterationsschritt $k$ die Differenz $u_{1}^{k}-w_{1}^{k}$ in $H^{1}\left(0, T ; V_{1,0}\right)$ ist, der Gleichung

$$
\frac{d}{d t}\left(u_{1}^{k}(t)-w_{1}^{k}(t), v\right)_{\Omega_{1}}+a_{1}\left(u_{1}^{k}(t)-w_{1}^{k}(t), v\right)=0
$$

für fast alle $t \in(0, T)$ und alle $v \in V_{1,0}$ sowie $u_{1}^{k}(0)-w_{1}^{k}(0)=0$ genügt und somit $u_{1}^{k}-w_{1}^{k}=0$ nach Satz 1.1.1 folgt.

Wiederum mit (5.12) folgt

$$
a_{2}(v, v)+\left\langle\left.\gamma v\right|_{\Gamma},\left.v\right|_{\Gamma}\right\rangle_{W} \geqslant \alpha_{2}\|v\|_{1, \Omega_{2}}^{2}
$$

für $\gamma \geqslant \gamma_{0}>0$ sowie

$$
\left|a_{2}(u, v)+\left\langle\left.\gamma u\right|_{\Gamma},\left.v\right|_{\Gamma}\right\rangle_{W}\right| \leqslant M_{2}\|u\|_{1, \Omega_{2}}\|v\|_{1, \Omega_{2}}+\left.\left.\|\gamma\|_{L^{\infty}(\Gamma)}|| u\right|_{\Gamma}\left\|{ }_{W}\right\| v\right|_{\Gamma} \|_{W}
$$

für alle $u, v \in V_{2}$. Nach dem Spursatz A.2.3 existiert eine Konstante $C_{t r, i}>0$, so daß

$$
\left\|\left.v\right|_{\Gamma}\right\|_{W} \leqslant C_{t r, i}\|v\|_{1, \Omega_{i}}
$$

für alle $v \in V_{i}, i=1,2$, gilt. Es ergibt sich also die Stetigkeitsaussage

$$
\left|a_{2}(u, v)+\left\langle\left.\gamma u\right|_{\Gamma},\left.v\right|_{\Gamma}\right\rangle_{W}\right| \leqslant\left(M_{2}+\|\gamma\|_{L^{\infty}(\Gamma)} C_{t r, 2}^{2}\right)\|u\|_{1, \Omega_{2}}\|v\|_{1, \Omega_{2}}
$$

für alle $u, v \in V_{2}$. Beachtet man, daß

$$
\begin{gathered}
\frac{d}{d t}\left(u_{2}^{k}(t), v\right)_{\Omega_{2}}+a_{2}\left(u_{2}^{k}(t), v\right)+\left\langle\left.\gamma u_{2}^{k}(t)\right|_{\Gamma},\left.v\right|_{\Gamma}\right\rangle_{W}=(f(t), v)_{\Omega_{2}}+\left(f(t),\left.r_{1} v\right|_{\Gamma}\right)_{\Omega_{2}} \\
-\frac{d}{d t}\left(u_{1}^{k}(t),\left.r_{1} v\right|_{\Gamma}\right)_{\Omega_{1}}-a_{1}\left(u_{1}^{k}(t),\left.r_{1} v\right|_{\Gamma}\right)+\left\langle\left.\gamma u_{1}^{k}(t)\right|_{\Gamma},\left.r_{1} v\right|_{\Gamma}\right\rangle_{W}
\end{gathered}
$$

für fast alle $t \in(0, T)$ und alle $v \in V_{2}$ 
die ersten beiden Gleichungen des Problems (5.14) gleichwertig ersetzt, so ist damit auch (5.14) nach Satz 1.1.1 eindeutig lösbar in $V_{2}$. Schließlich gilt $\left.u_{2}^{k}\right|_{\Gamma} \in Z$ nach Lemma 5.2.3, also auch $g^{k}=\left.\sigma u_{2}^{k}\right|_{\Gamma}+(1-\sigma) g^{k-1} \in Z$. 


\section{Kapitel 6}

\section{Konvergenzanalyse eines Dirichlet-Robin-Verfahrens}

Ziel dieses Kapitels ist ein Konvergenzresultat für das in Kapitel 5 konstruierte DirichletRobin-Verfahren für zwei Teilgebiete. Dazu geben wir Iterationsvorschriften für den Fehler zwischen einer Lösung dieses Verfahrens und der Lösung des Zweigebietproblems (5.10) auf dem Austauschrand mittels geeigneter Operatoren an.

Im zweiten Abschnitt führen wir Steklov-Poincaré-Operatoren für parabolische Probleme ein. Der Zusammenhang zwischen diesen Operatoren und dem Dirichlet-Robin-Verfahren ermöglicht den Nachweis linearer Konvergenz des Verfahres auf Grundlage einer bekannten abstrakten Konvergenzaussage im letzten Abschnitt. Allerdings kommen wir dabei nicht ohne eine Hypothese bezüglich der Steklov-Poincaré-Operatoren aus, die sinnvoll erscheint, deren Beweis jedoch offen bleibt.

\subsection{Fehlergleichungen}

Das iterative Schema ist so gewählt, daß die konstante Folge $\left(\left(u_{1}^{k}, u_{2}^{k}\right)\right)_{k \in \mathbb{N}}$ mit $u_{i}^{k}:=u_{i}, k \in \mathbb{N}$, Lösung zum Startwert $g^{0}:=\left.u_{1}\right|_{\Gamma}$ ist (bei beliebigem Relaxationsparameter $\sigma$ ), sofern $\left(u_{1}, u_{2}\right)$ dem Zweigebietsproblem (5.10) genügt.

Für die Differenzenfolge $\left(\left(\tilde{u}_{1}^{k}, \tilde{u}_{2}^{k}\right)\right)_{k \in \mathbb{N}}$ mit $\tilde{u}_{i}^{k}:=u_{i}^{k}-u_{i}, i=1,2$, einer Lösung $\left(\left(u_{1}^{k}, u_{2}^{k}\right)\right)_{k \in \mathbb{N}}$ von (5.13) - (5.15) und einer Lösung $\left(u_{1}, u_{2}\right)$ von (5.10) mit identischen Anfangswerten gelten die Fehlergleichungen

$$
\frac{d}{d t}\left(\tilde{u}_{1}^{k}(t), v\right)_{\Omega_{1}}+a_{1}\left(\tilde{u}_{1}^{k}(t), v\right)=0 \quad \text { für alle } v \in V_{1,0}
$$

und

$$
\begin{gathered}
\frac{d}{d t}\left(\tilde{u}_{2}^{k}(t), v\right)_{\Omega_{2}}+a_{2}\left(\tilde{u}_{2}^{k}(t), v\right)+\left\langle\left.\gamma \tilde{u}_{2}^{k}(t)\right|_{\Gamma},\left.v\right|_{\Gamma}\right\rangle_{W}=-\frac{d}{d t}\left(\tilde{u}_{1}^{k}(t),\left.r_{1} v\right|_{\Gamma}\right)_{\Omega_{1}} \\
-a_{1}\left(\tilde{u}_{1}^{k}(t),\left.r_{1} v\right|_{\Gamma}\right)+\left\langle\left.\gamma \tilde{u}_{1}^{k}(t)\right|_{\Gamma},\left.v\right|_{\Gamma}\right\rangle_{W} \quad \text { für alle } v \in V_{2}
\end{gathered}
$$

jeweils für fast alle $t \in(0, T)$. Für die Anfangswerte der Differenzenfolge gilt 


$$
\tilde{u}_{i}^{k}(0)=0, \quad i=1,2 .
$$

Für die Konvergenzuntersuchung dieses Kapitels ersetzen wir die zweite Fehlergleichung (6.2) durch eine schwächere Form mit erweitertem Austauschrandterm:

$$
\begin{gathered}
\int_{0}^{T}\left\langle\left(\tilde{u}_{2}^{k}\right)^{\prime}(t), v(t)\right\rangle_{V_{2}^{*} \times V_{2}}+a_{2}\left(\tilde{u}_{2}^{k}(t), v(t)\right) d t+\left\langle\left.\gamma \tilde{u}_{2}^{k}\right|_{\Gamma},\left.v\right|_{\Gamma}\right\rangle_{Z}=\int_{0}^{T}-\left\langle\left(\tilde{u}_{1}^{k}(t)\right)^{\prime},\left.r_{1} v\right|_{\Gamma}(t)\right\rangle_{V_{2}^{*} \times V_{2}} \\
-a_{1}\left(\tilde{u}_{1}^{k}(t),\left.r_{1} v\right|_{\Gamma}(t)\right) d t+\left\langle\left.\gamma \tilde{u}_{1}^{k}\right|_{\Gamma},\left.v\right|_{\Gamma}\right\rangle_{Z} \quad \text { für alle } v \in H^{1}\left(0, T ; V_{2}\right) .
\end{gathered}
$$

Dabei bezeichnet $\langle\cdot, \cdot\rangle_{Z}$ das Skalarprodukt auf $Z=L^{2}(0, T ; W) \cap H^{\frac{1}{4}}\left(0, T ; L^{2}(\Gamma)\right)$ (vergl. A.3), also

$$
\langle u(t), v(t)\rangle_{Z}=\int_{0}^{T}\langle u(t), v(t)\rangle_{W} d t+\int_{0}^{T} \frac{\langle u(t)-u(\tau), v(t)-v(\tau)\rangle_{L^{2}(\Gamma)}}{|t-\tau|^{\frac{3}{2}}} d \tau d t, \quad u, v \in Z .
$$

Für $u \in Z$ und jede konstante Funktion $v \in Z$ mit $v(t)=w$ für ein $w \in W$ fast überall in $(0, T)$ gilt offensichtlich $\langle u, v\rangle_{Z}=\int_{0}^{T}\langle u(t), w\rangle_{W} d t$. Daher hat für jede konstante Funktion $v \in V_{2}^{(0, T)} \subseteq H^{1}\left(0, T ; V_{2}\right)$ die Gleichung (6.3) die Gestalt

$$
\begin{aligned}
\int_{0}^{T}\left\langle\left(\tilde{u}_{2}^{k}\right)^{\prime}(t), v(t)\right\rangle_{V_{2}^{*} \times V_{2}}+a_{2}\left(\tilde{u}_{2}^{k}(t), v(t)\right)+\left\langle\left.\gamma \tilde{u}_{2}^{k}(t)\right|_{\Gamma},\left.v\right|_{\Gamma}(t)\right\rangle_{W} d t= \\
\quad-\int_{0}^{T}\left\langle\tilde{u}_{1}^{k}(t),\left.r_{1} v\right|_{\Gamma}(t)\right\rangle_{V_{1}^{*} \times V_{1}}+a_{1}\left(\tilde{u}_{1}^{k}(t),\left.r_{1} v\right|_{\Gamma}(t)\right)+\left\langle\left.\gamma \tilde{u}_{1}^{k}(t)\right|_{\Gamma},\left.v\right|_{\Gamma}(t)\right\rangle_{W} d t
\end{aligned}
$$

was einer schwachen (integralen) Formulierung der punktweise geltenden Fehlergleichung (6.2) entspricht.

Die oben gemachte Ersetzung der Fehlergleichung entspricht einer analogen Modifikation der des Dirichlet-Robin-Alghorithmus (5.13) - (5.15), der Zweigebietsformulierung (5.10) sowie der Bestimmungsgleichung (5.16) der Fortsetzungsoperatoren $E_{i}, i=1,2$.

Die Fehlergleichung (6.3) motiviert die folgenden Definitionen der Operatoren $K$ und $K_{\sigma}$, die wesentliche Hilfsmittel der Konvergenzanalyse dieses Abschnittes darstellen.

Mit $K$ bezeichnen wir die Abbildung $w \mapsto K w$ auf $Z$, die durch

$$
K w(t):=\left.\tilde{u}_{2}(t)\right|_{\Gamma}
$$

für fast jedes $t \in(0, T)$ definiert wird, wobei $\tilde{u}_{2}=\tilde{u}_{2}(w) \in H^{1}\left(0, T ; V_{2}\right)$ bestimmt ist durch

$$
\left\{\begin{array}{l}
\int_{0}^{T}\left\langle\left(\tilde{u}_{2}\right)^{\prime}(t), v(t)\right\rangle_{V_{2}^{*} \times V_{2}}+a_{2}\left(\tilde{u}_{2}(t), v(t)\right) d t+\left\langle\left.\gamma \tilde{u}_{2}\right|_{\Gamma},\left.v\right|_{\Gamma}\right\rangle_{Z}= \\
\quad-\int_{0}^{T}\left\langle\left(E_{1} w\right)^{\prime}(t),\left.E_{1} v\right|_{\Gamma}(t)\right\rangle_{V_{1}^{*} \times V_{1}}+a_{1}\left(E_{1} w(t),\left.E_{1} v\right|_{\Gamma}(t)\right) d t+\left\langle\gamma w,\left.v\right|_{\Gamma}\right\rangle_{Z} \\
\quad \text { für alle } v \in H^{1}\left(0, T ; V_{2}\right) \\
\tilde{u}_{2}(0)=0 \quad \text { in } L^{2}\left(\Omega_{2}\right)
\end{array}\right.
$$


Mit Hilfe des Operators $K$ definieren wir zu jedem $\sigma>0$

$$
K_{\sigma}:\left\{\begin{aligned}
Z & \rightarrow Z \\
w & \mapsto K_{\sigma} w:=\sigma K w+(1-\sigma) w .
\end{aligned}\right.
$$

Betrachtet man wie oben eine Differenzenfolge $\left(\left(\tilde{u}_{1}^{k}, \tilde{u}_{2}^{k}\right)\right)_{k \in \mathbb{N}}$ zum Relaxationsparameter $\sigma>0$, so liefert der Operator $K$ zur Spur von $\tilde{u}_{1}^{k}$ auf $\Gamma$ die Spur von $\tilde{u}_{2}^{k}$ auf $\Gamma$ im Iterationsschritt $k \in \mathbb{N}$.

Entscheidend wird sein, daß man mit Hilfe des Operators $K_{\sigma}$ eine Iterationsvorschrift für die Differenz $\left.\tilde{u}_{1}^{k}\right|_{\Gamma}$ angeben kann:

$$
\left.\tilde{u}_{1}^{k+1}\right|_{\Gamma}=\left.K_{\sigma} \tilde{u}_{1}^{k}\right|_{\Gamma}
$$

Außerdem reproduziert sich die Relaxationsgleichung (5.15) für $\left(\left(\tilde{u}_{1}^{k}, \tilde{u}_{2}^{k}\right)\right)_{k \in \mathbb{N}^{*}}$. Dies sind die Aussagen des folgenden Lemmas.

Lemma 6.1.1 Es sei $\left(u_{1}, u_{2}\right)$ eine Lösung des Zweigebietsformulierung (5.10) und $\left(\left(u_{1}^{k}, u_{2}^{k}\right)\right)_{k \in \mathbb{N}}$ eine Lösung des Dirichlet-Robin-Alghorithmus (5.13) - (5.15) zu identischen Anfangswerten. Dann gilt für die Differenzen $\tilde{u}_{i}^{k}:=u_{i}^{k}-u_{i}, i=1,2, k \in \mathbb{N}$,

$$
\begin{aligned}
\left.\tilde{u}_{1}^{k+1}\right|_{\Gamma} & =\left.\sigma \tilde{u}_{2}^{k}\right|_{\Gamma}+\left.(1-\sigma) \tilde{u}_{1}^{k}\right|_{\Gamma} \\
\left.\tilde{u}_{2}^{k}\right|_{\Gamma} & =\left.K \tilde{u}_{1}^{k}\right|_{\Gamma} \\
\left.\tilde{u}_{i}^{k+1}\right|_{\Gamma} & =\left.K_{\sigma} \tilde{u}_{i}^{k}\right|_{\Gamma}, \quad i=1,2 .
\end{aligned}
$$

Beweis. $\quad$ Nach Voraussetzung gilt $\tilde{u}_{i}^{k}=u_{i}^{k}-u_{i} \in H^{1}\left(0, T ; V_{i}\right), k \in \mathbb{N}$, somit folgt $\left.\tilde{u}_{i}^{k}\right|_{\Gamma} \in Z$ nach Lemma 5.2.3. Wegen der Randbedingungen $(5.10)_{2}$ und $(5.13)_{2}$ sowie der Relaxationsgleichung (5.15) erhält man

$$
\begin{aligned}
\left.\tilde{u}_{1}^{k+1}\right|_{\Gamma} & =\left.\sigma u_{2}^{k}\right|_{\Gamma}+\left.(1-\sigma) u_{1}^{k}\right|_{\Gamma}-\left.u_{1}\right|_{\Gamma} \\
& =\left.\sigma\left(u_{2}^{k}-u_{2}\right)\right|_{\Gamma}+\left.(1-\sigma)\left(u_{1}^{k}-u_{1}\right)\right|_{\Gamma} \\
& =\left.\sigma \tilde{u}_{2}^{k}\right|_{\Gamma}+\left.(1-\sigma) \tilde{u}_{1}^{k}\right|_{\Gamma}
\end{aligned}
$$

(in Z), und dies ist die Gleichung (6.6).

Der Nachweis der Gleichung (6.7) beruht auf der Gleichung $\tilde{u}_{1}^{k}=\left.E_{1} \tilde{u}_{1}^{k}\right|_{\Gamma}$ nach Definition von $E_{1}$. Aufgrund der Fehlergleichung (6.3) folgt

$$
\begin{aligned}
& \int_{0}^{T}\left\langle\left(\tilde{u}_{2}^{k}\right)^{\prime}(t), v(t)\right\rangle_{V_{2}^{*} \times V_{2}}+a_{2}\left(\tilde{u}_{2}^{k}(t), v(t)\right) d t+\left\langle\left.\gamma \tilde{u}_{2}^{k}\right|_{\Gamma},\left.v\right|_{\Gamma}\right\rangle_{Z}= \\
& \quad-\int_{0}^{T}\left\langle\left(\left.E_{1} \tilde{u}_{1}^{k}\right|_{\Gamma}\right)^{\prime}(t),\left.E_{1} v\right|_{\Gamma}(t)\right\rangle_{V_{1}^{*} \times V_{1}}+a_{1}\left(\left.E_{1} \tilde{u}_{1}^{k}\right|_{\Gamma}(t),\left.E_{1} v\right|_{\Gamma}(t)\right) d t+\left\langle\left.\gamma \tilde{u}_{1}^{k}\right|_{\Gamma},\left.v\right|_{\Gamma}\right\rangle_{Z}
\end{aligned}
$$


für alle $v \in H^{1}\left(0, T, V_{2}\right)$, nach Definition von $K$ ist daher $\left.K \tilde{u}_{1}^{k}\right|_{\Gamma}=\left.\tilde{u}_{2}^{k}\right|_{\Gamma}$.

Die Gleichung (6.8) folgt für $i=1$ nach Definition von $K_{\sigma}$ unmittelbar aus den bereits bewiesenen Gleichungen (6.6) und (6.7). Für $i=2$ schließt man mit diesen sowie der Linearität von $K$

$$
\begin{aligned}
\left.\tilde{u}_{2}^{k+1}\right|_{\Gamma} & =\left.K \tilde{u}_{1}^{k+1}\right|_{\Gamma} \\
& =\left.\sigma K \tilde{u}_{2}^{k}\right|_{\Gamma}+\left.(1-\sigma) K \tilde{u}_{1}^{k}\right|_{\Gamma} \\
& =\left.\sigma K \tilde{u}_{2}^{k}\right|_{\Gamma}+\left.(1-\sigma) \tilde{u}_{2}^{k}\right|_{\Gamma}=\left.K_{\sigma} \tilde{u}_{2}^{k}\right|_{\Gamma} .
\end{aligned}
$$

Um die Konvergenz $\left.\left.u_{1}^{k}\right|_{\Gamma} \rightarrow u_{1}\right|_{\Gamma}$ für $k \rightarrow \infty$ zu zeigen, reicht es also aus, ein $\sigma$ anzugeben, so daß die Abbildung $K_{\sigma}$ kontrahiert. Im weiteren Verlauf (siehe (i) des Beweises zu Satz 6.3.3) wird sich herausstellen, daß die Konvergenz $\left.\left.u_{1}^{k}\right|_{\Gamma} \rightarrow u_{1}\right|_{\Gamma}$ auch die Konvergenz $\left.\left.u_{2}^{k}\right|_{\Gamma} \rightarrow u_{2}\right|_{\Gamma}$ nach sich zieht.

\subsection{Steklov-Poincaré-Operatoren für parabolische Probleme}

In Anlehnung an den Steklov-Poincaré-Operator für elliptische Probleme (siehe etwa [58]) definieren wir geeignete Operatoren für den vorliegenden zeitabhängigen Fall. Für jedes $v \in Z$ sei $S_{i}: w \mapsto\left\langle S_{i} v, w\right\rangle$ der Operator aus $Z^{*}$ mit (siehe Lemma 6.2.1)

$$
\left\langle S_{i} v, w\right\rangle \quad:=\int_{0}^{T}\left\langle\left(E_{i} v\right)^{\prime}(t), E_{i} w(t)\right\rangle_{V_{i}^{*} \times V_{i}}+a_{i}\left(E_{i} v(t), E_{i} w(t)\right) d t, \quad i=1,2
$$

und $S$ der Summenoperator beider Operatoren, also

$$
\langle S v, w\rangle:=\left\langle S_{1} v, w\right\rangle+\left\langle S_{2} v, w\right\rangle
$$

Daran anschließend definieren wir für jede Funktion $\gamma \in L^{\infty}(\Gamma)$ die Operatoren $S_{1}^{(\gamma)}$ und $S_{2}^{(\gamma)}$ durch

$$
\begin{aligned}
& \left\langle S_{1}^{(\gamma)} v, w\right\rangle:=\left\langle S_{1} v, w\right\rangle-\langle\gamma v, w\rangle_{Z} \\
& \left\langle S_{2}^{(\gamma)} v, w\right\rangle:=\left\langle S_{2} v, w\right\rangle+\langle\gamma v, w\rangle_{Z} .
\end{aligned}
$$


$S_{i}$ läßt sich dann - bezüglich des zweiten Arguments - mit beliebigem Fortsetzungsoperator $R_{i}: Z \rightarrow H^{1}\left(0, T ; V_{i}\right)$ mit $\left.R_{i} w\right|_{\Gamma}=w, R_{i}(0)=0$ formulieren:

$$
\begin{aligned}
\left\langle S_{i} v, w\right\rangle= & \int_{0}^{T}\left\langle\left(E_{i} v\right)^{\prime}(t), E_{i} w(t)\right\rangle_{V_{i}^{*} \times V_{i}}+a_{i}\left(E_{i} v(t), E_{i} w(t)\right) d t \\
= & \int_{0}^{T}\left\langle\left(E_{i} v\right)^{\prime}(t), R_{i} w(t)\right\rangle_{V_{i}^{*} \times V_{i}}+a_{i}\left(E_{i} v(t), R_{i} w(t)\right) d t \\
& +\int_{0}^{T}\left\langle\left(E_{i} v\right)^{\prime}(t), E_{i} w(t)-R_{i} w(t)\right\rangle_{V_{i}^{*} \times V_{i}}+a_{i}\left(E_{i} v(t), E_{i} w(t)-R_{i} w(t)\right) d t \\
= & \int_{0}^{T}\left\langle\left(E_{i} v\right)^{\prime}(t), R_{i} w(t)\right\rangle_{V_{i}^{*} \times V_{i}}+a_{i}\left(E_{i} v(t), R_{i} w(t)\right) d t
\end{aligned}
$$

da $E_{i} w-R_{i} w \in V_{i, 0}$ für faßt alle $t \in(0, T)$ und $\left(E_{i} w-R_{i} w\right)(0)=0$ nach Definition von $E_{i}$, $i=1,2$.

Für die Operatoren $S_{i}, i=1,2$, erhält man mit (5.12) und dem Spursatz A.2.3

$$
\begin{aligned}
\left\langle S_{i} w, w\right\rangle & =\int_{0}^{T} \frac{1}{2} \frac{d}{d t}\left\|E_{i} w(t)\right\|_{0, \Omega_{i}}^{2}+a_{i}\left(E_{i} w(t), E_{i} w(t)\right) d t \\
& \geqslant\left\|E_{i} w(T)\right\|_{0, \Omega_{i}}^{2}+\int_{0}^{T} \alpha_{i}\left\|E_{i} w(t)\right\|_{1, \Omega_{i}}^{2} d t \\
& \geqslant\left\|E_{i} w(T)\right\|_{0, \Omega_{i}}^{2}+\alpha_{i} C_{t r, i} \int_{0}^{T}\|w\|_{W}^{2} d t \\
& =\left\|E_{i} w(T)\right\|_{0, \Omega_{i}}^{2}+\alpha_{i} C_{t r, i}\|w\|_{L^{2}(0, T ; W)}^{2}
\end{aligned}
$$

mit einer Konstante $C_{t r, i}=C_{t r, i}\left(\Omega_{i}\right)>0$.

Wesentliche Eigenschaften der Operatoren $S_{1}^{(\gamma)}$ und $S_{2}^{(\gamma)}$ hält das folgende Lemma fest.

Lemma 6.2.1 $S_{1}^{(\gamma)}$ und $S_{2}^{(\gamma)}$ sind lineare, stetige Abbildungen von $Z$ nach $Z^{*}$. Zudem ist $S_{2}^{(\gamma)}$ koerziv, sofern $\gamma \geqslant \gamma_{0}>0$.

Beweis. Zunächst wird die Existenz der Integrale durch $E_{i} w \in L^{2}\left(0, T ; V_{i}\right),\left(E_{i} w\right)^{\prime} \in$ $L^{2}\left(0, T ; V_{i}^{*}\right)$ und durch die Stetigkeit von $a_{i}$ gemäß (5.12) gesichert. Für $v, w \in Z$ ist nämlich

$$
\left|\left\langle S_{i} v, w\right\rangle\right| \leqslant \int_{0}^{T}\left\|\left(E_{i} v\right)^{\prime}(t)\right\|_{V_{i}^{*}}\left\|E_{i} w(t)\right\|_{V_{i}}+M_{i}\left\|E_{i} v(t)\right\|_{V_{i}}\left\|E_{i} w(t)\right\|_{V_{i}} d t .
$$

Zieht man die Hölder-Ungleichung für Integrale heran, so ergibt sich daher

$$
\begin{aligned}
\left|\left\langle S_{i} v, w\right\rangle\right| \leqslant & \left(\int_{0}^{T}\left\|\left(E_{i} v\right)^{\prime}(t)\right\|_{V_{i}^{*}}^{2} d t\right)^{\frac{1}{2}}\left(\int_{0}^{T}\left\|E_{i} w(t)\right\|_{V_{i}}^{2} d t\right)^{\frac{1}{2}} \\
& +M_{i}\left(\int_{0}^{T}\left\|E_{i} v(t)\right\|_{V_{i}}^{2} d t\right)^{\frac{1}{2}}\left(\int_{0}^{T}\left\|E_{i} w(t)\right\|_{V_{i}}^{2} d t\right)^{\frac{1}{2}} \\
= & \left\|\left(E_{i} v\right)^{\prime}\right\|_{L^{2}\left(0, T ; V_{i}^{*}\right)}\left\|E_{i} w\right\|_{L^{2}\left(0, T ; V_{i}\right)} \\
& +M_{i}\left\|E_{i} v\right\|_{L^{2}\left(0, T ; V_{i}\right)}\left\|E_{i} w\right\|_{L^{2}\left(0, T ; V_{i}\right)} .
\end{aligned}
$$


Zudem gilt mit der Cauchy-Schwarz-Ungleichung für $\langle\cdot, \cdot\rangle_{Z}$

$$
\left|\langle\gamma v, w\rangle_{Z}\right| \leqslant\|\gamma\|_{L^{\infty}(\Gamma)}\|v\|_{Z}\|w\|_{Z}
$$

Zum Nachweis der Stetigkeit zieht man wiederum die Ungleichung (6.14) heran. Mit Hilfe der Abschätzungen aus Lemma 5.2.3

$$
\left\|E_{i} w\right\|_{H^{1}\left(0, T ; V_{i}\right)} \leqslant C_{E_{i}}\|w\|_{Z}, \quad w \in Z,
$$

folgert man

$$
\begin{aligned}
\left|\left\langle S_{i} v, w\right\rangle\right| & \leqslant\left\|E_{i} v\right\|_{H^{1}\left(0, T ; V_{i}\right)}\left\|E_{i} w\right\|_{H^{1}\left(0, T ; V_{i}\right)}+M_{i}\left\|E_{i} v\right\|_{H^{1}\left(0, T ; V_{i}\right)}\left\|E_{i} w\right\|_{H^{1}\left(0, T ; V_{i}\right)} \\
& \leqslant\left(1+M_{i}\right) C_{E_{i}}^{2}\|v\|_{Z}\|w\|_{Z}
\end{aligned}
$$

für $i=1,2$. Dies liefert zusammen mit (6.15) und der Bezeichnung $m_{i}:=\left(1+M_{i}\right) C_{E_{i}}^{2}$ die Stetigkeitsaussage

$$
\left|\left\langle S_{i}^{(\gamma)} v, w\right\rangle\right| \leqslant\left(m_{i}+\|\gamma\|_{L^{\infty}(\Gamma)}\right)\|v\|_{Z}\|w\|_{Z}
$$

für $i=1,2$.

Schließlich folgt wegen $(6.13)$

$$
\left\langle S_{2}^{(\gamma)} w, w\right\rangle \geqslant\left\|E_{2} w(T)\right\|_{0, \Omega_{2}}^{2}+\alpha_{2} C_{t r, 2}\|w\|_{L^{2}(0, T ; W)}^{2}+\gamma_{0}\|w\|_{Z}^{2} \geqslant \gamma_{0}\|w\|_{Z}^{2}
$$

also die Koerzitivität von $S_{2}^{(\gamma)}$ für $\gamma \geqslant \gamma_{0}>0$.

Für den Fall $\gamma \geqslant \gamma_{0}>0$ schafft Lemma 6.2.1 die Voraussetzungen zur Anwendung des LaxMilgram-Theorems, wonach $S_{2}^{(\gamma)}$ bijektiv ist. Aus demselben Lemma folgt zudem, daß dann durch

$$
\langle v, w\rangle_{2}:=\frac{1}{2}\left(\left\langle S_{2}^{(\gamma)} v, w\right\rangle+\left\langle S_{2}^{(\gamma)} w, v\right\rangle\right)
$$

ein Skalarprodukt auf $Z$ definiert wird und daß die induzierte Norm ||$\cdot|| \mid$ mit

$$
\|w\|:=\sqrt{\left\langle S_{2}^{(\gamma)} w, w\right\rangle}
$$

äquivalent zur Norm $\|\cdot\|_{Z}$ ist, nämlich per

$$
\sqrt{\gamma_{0}}\|w\|_{Z} \leqslant\|w\| \leqslant \sqrt{m_{i}+\|\gamma\|_{L^{\infty}(\Gamma)}}\|w\|_{Z}
$$

Den Zusammenhang zwischen den oben eingeführten Steklov-Poincaré-Operatoren $S_{1}^{(\gamma)}$ und $S_{2}^{(\gamma)}$ sowie unserem Dirichlet-Robin-Algorithmus (5.13) - (5.15) stellt das folgende Lemma mit der Darstellung von $K_{\sigma}$ durch $S_{1}^{(\gamma)}$ und $S_{2}^{(\gamma)}$ her. Daraus ergibt sich die Bedeutung der Steklov-Poincaré-Operatoren für die nachfolgende Konvergenzuntersuchung. 
Lemma 6.2.2 Für den Operator $K_{\sigma}, \sigma>0$, laut (6.5) gilt

$$
K_{\sigma}=1-\sigma\left(S_{2}^{(\gamma)}\right)^{-1} S
$$

mit $S_{2}^{(\gamma)}$ und $S$ gemäß (6.12) bzw. (6.10).

Beweis. $\quad$ Es sei $w \in Z$. Dann gilt für die Lösung $\tilde{u}_{2}=\tilde{u}_{2}(w)$ von (6.4)

$$
\left\{\begin{array}{c}
\int_{0}^{T}\left\langle\tilde{u}_{2}^{\prime}(t), E_{2} v(t)\right\rangle_{V_{2}^{*} \times V_{2}}+a_{2}\left(\tilde{u}_{2}(t), E_{2} v(t)\right) d t+\left\langle\left.\gamma \tilde{u}_{2}\right|_{\Gamma}, v\right\rangle_{Z}= \\
\quad-\int_{0}^{T}\left\langle\left(E_{1} w\right)^{\prime}(t), E_{1} v(t)\right\rangle_{V_{1}^{*} \times V_{1}}+a_{1}\left(E_{1} w(t), E_{1} v(t)\right) d t+\langle\gamma w(t), v\rangle_{Z} \\
\quad \text { für fast alle } t \in(0, T) \text { und alle } v \in Z, \\
\int_{0}^{T}\left\langle\tilde{u}_{2}(t), v(t)\right\rangle_{V_{2}^{*} \times V_{2}}+a_{2}\left(\tilde{u}_{2}(t), v(t)\right) d t=0 \\
\quad \text { für fast alle } t \in(0, T) \text { und alle } v \in H^{1}\left(0, T ; V_{2,0}\right) .
\end{array}\right.
$$

Wegen $(6.21)_{2}$ ergibt sich nach Definition des Operators $E_{2}$

$$
\tilde{u}_{2}=\left.E_{2} \tilde{u}_{2}\right|_{\Gamma}=E_{2} K w .
$$

In Verbindung mit $(6.21)_{1}$ folgt daher für jedes $z \in Z$

$$
\begin{aligned}
\left\langle S_{2}^{(\gamma)} K w, z\right\rangle= & \int_{0}^{T}\left\langle\left(E_{2} K w\right)^{\prime}(t), E_{2} z(t)\right\rangle_{V_{2}^{*} \times V_{2}}+a_{2}\left(E_{2} K w(t), E_{2} z(t)\right) d t \\
& \quad+\langle\gamma K w, z\rangle_{Z} \\
= & \int_{0}^{T}\left\langle\tilde{u}_{2}^{\prime}(t), E_{2} z(t)\right\rangle_{V_{2}^{*} \times V_{2}}+a_{2}\left(\tilde{u}_{2}(t), E_{2} z(t)\right) d t+\left\langle\left.\gamma \tilde{u}_{2}\right|_{\Gamma}, z\right\rangle_{Z} \\
= & -\int_{0}^{T}\left\langle\left(E_{1} w\right)^{\prime}(t), E_{1} z(t)\right\rangle_{V_{1}^{*} \times V_{1}}+a_{1}\left(E_{1} w(t), E_{1} z(t)\right)-\langle\gamma w, z\rangle_{Z} d t \\
= & -\left\langle S_{1}^{(\gamma)} w, z\right\rangle,
\end{aligned}
$$

was gleichwertig ist $\mathrm{zu}$

$$
\left\langle S_{2}^{(\gamma)}(K w-w), z\right\rangle=-\left\langle S_{1}^{(\gamma)} w, z\right\rangle-\left\langle S_{2}^{(\gamma)} w, z\right\rangle=-\langle S w, z\rangle
$$

Da $S_{2}^{(\gamma)}$ unter Annahme von (6.25) invertierbar ist, folgt $\sigma K w-\sigma w=-\sigma\left(S_{2}^{(\gamma)}\right)^{-1} S w$ für jedes $\sigma \in \mathbb{R}$, mithin die behauptete Darstellung.

\subsection{Ein Konvergenzresultat}

Bezogen auf die soeben eingeführten Operatoren $S_{1}^{(\gamma)}, S_{2}^{(\gamma)}$ soll die folgende abstrakte Konvergenzaussage aus [2] zur Anwendung kommen. 
Satz 6.3.1 Es sei $X$ ein reeller Hilbert-Raum, $Q_{1}, Q_{2}$ seien lineare Operatoren $X \rightarrow X^{*}$, und es gebe positive Konstanten $l_{1}, l_{2}$, so daß

$$
\left\langle Q_{i} v, w\right\rangle \leqslant l_{i}\|v\|_{X}\|w\|_{X} \quad v, w \in X
$$

für $i=1,2$ gilt, sowie positive Konstanten $\mu_{2}, \kappa$, so daß

$$
\left\langle Q_{2} w, w\right\rangle \geqslant \mu_{2}\|w\|_{X}^{2} \quad w \in X
$$

und

$$
\left\langle Q_{2} w, Q_{2}^{-1} Q w\right\rangle+\langle Q w, w\rangle \geqslant \kappa\|w\|_{X}^{2} \quad w \in X,
$$

gilt mit $Q:=Q_{1}+Q_{2}$. Dann wird durch $\langle v, w\rangle_{Q_{2}}:=\frac{1}{2}\left(\left\langle Q_{2} v, w\right\rangle+\left\langle Q_{2} w, v\right\rangle\right)$ ein Skalarprodukt auf $X$ definiert, und die Abbildung

$$
w \mapsto\left(1-\sigma Q_{2}^{-1} Q\right) w
$$

ist für jeden Parameter $\sigma$, der

$$
0<\sigma<\frac{\kappa \mu_{2}^{2}}{l_{2}\left(l_{1}+l_{2}\right)}
$$

genügt, eine Kontraktion auf $X$ bezüglich der durch $\langle\cdot, \cdot\rangle_{Q_{2}}$ induzierten Norm.

Für die nachfolgende Anwendung von Satz 6.3.1 machen wir die Annahme, daß es ein $\alpha_{S}>0$ gibt mit

$$
\langle S w, w\rangle=\sum_{i \in \underline{2}} \int_{0}^{T}\left\langle\left(E_{i} w\right)^{\prime}(t), E_{i} w(t)\right\rangle_{V_{i}^{*} \times V_{i}}+a_{i}\left(E_{i} w(t), E_{i} w(t)\right) d t \geqslant \alpha_{S}\|w\|_{Z}^{2}
$$

für alle $w \in Z$, also die Annahme der Koerzivität von $S$. Nach Definition von $E_{i}$ ist $E_{i} w \in$ $H^{1}\left(0, T ; V_{i}\right)$, und gemäß [45], Seite 83-84, ist $H^{1}\left(0, T ; V_{i}\right) \cong Z$. Dies legt nahe, daß die Annahme (6.25) sinnvoll ist. Vergleicht man andererseits die Annahme (6.25) mit der rechte Seite der letzten Ungleichung in (6.13), so fehlt, läßt man den Zeitableitungsterm außer acht, gerade der Term $\|w\|_{H^{\frac{1}{4}}\left(0, T ; L^{2}(\Gamma)\right)}$. Leider konnten wir ein entsprechendes Ergebnis weder beweisen noch in der Literatur finden, so daß wir den Beweis der Annahme offen lassen müssen.

Dank der Darstellung von $K_{\sigma}$ gemäß Lemma 6.2.2 durch die Steklov-Poincaré-Operatoren $S_{1}^{(\gamma)}$ und $S_{2}^{(\gamma)}$ sind wir in der Lage, auf der Grundlage von Annahme (6.25) mittels Satz 6.3.1 zu zeigen, daß $K_{\sigma}$ kontrahiert.

Lemma 6.3.2 Die Gültigkeit von (6.25) sei angenommen. Dann lassen sich Konstanten $\gamma_{0}>0$ und $\sigma^{*} \in(0,1]$ angeben, so daß zu jedem $\sigma \in\left(0, \sigma^{*}\right)$ eine Konstante $C_{\sigma}<1$ existiert, für die

$$
\left\|K_{\sigma} w\right\| \leqslant C_{\sigma}\|w\| \| \quad \text { für alle } w \in Z
$$

gilt, sofern $\gamma \in L^{\infty}(\Gamma)$ und $\gamma \geqslant \gamma_{0}$. Dabei ist $\|\cdot\| \|$ die durch $\gamma$ parametrisierte Norm (6.19). 
Beweis. Um Voraussetzung (6.24) mit $Q_{i}=S_{i}^{(\gamma)}$ zu verifizieren, setzen wir $z:=\left(S_{2}^{(\gamma)}\right)^{-1} S w$. Dann ist

$$
\begin{aligned}
\left\langle S_{2}^{(\gamma)} w,\left(S_{2}^{(\gamma)}\right)^{-1} S w\right\rangle+\langle S w, w\rangle & =2\langle S w, w\rangle+\left\langle S_{2}^{(\gamma)} w, z\right\rangle-\langle S w, w\rangle \\
& =2\langle S w, w\rangle+\left\langle S_{2}^{(\gamma)} w, z\right\rangle-\left\langle S_{2}^{(\gamma)} z, w\right\rangle \\
& \geqslant 2\langle S w, w\rangle-\left|\left\langle S_{2}^{(\gamma)} w, z\right\rangle-\left\langle S_{2}^{(\gamma)} z, w\right\rangle\right| .
\end{aligned}
$$

Die Abweichung $\left|\left\langle S_{2}^{(\gamma)} w, z\right\rangle-\left\langle S_{2}^{(\gamma)} z, w\right\rangle\right| \operatorname{des}$ Operators $\left\langle S_{2}^{(\gamma)} \cdot, \cdot\right\rangle$ von der Symmetrie läßt sich abschätzen durch

$$
\begin{aligned}
\left|\left\langle S_{2}^{(\gamma)} w, z\right\rangle-\left\langle S_{2}^{(\gamma)} z, w\right\rangle\right|= & \mid \int_{0}^{T}\left\langle\left(E_{2} w\right)^{\prime}(t), E_{2} z(t)\right\rangle_{V_{2}^{*} \times V_{2}}+a_{2}\left(E_{2} w(t), E_{2} z(t)\right) \\
& -\left\langle\left(E_{2} z\right)^{\prime}(t), E_{2} w(t)\right\rangle_{V_{2}^{*} \times V_{2}}-a_{2}\left(E_{2} z(t), E_{2} w(t)\right) d t \mid \\
= & \mid \int_{0}^{T}\left\langle\left(E_{2} w\right)^{\prime}(t), E_{2} z(t)\right\rangle_{V_{2}^{*} \times V_{2}}-\left\langle\left(E_{2} z\right)^{\prime}(t), E_{2} w(t)\right\rangle_{V_{2}^{*} \times V_{2}} \\
& +\int_{\Omega} b \cdot\left(E_{2} z(t) \nabla E_{2} w(t)-E_{2} w(t) \nabla E_{2} z(t)\right) d x d t \mid \\
\leqslant & \int_{0}^{T}\left\|\left(E_{2} w\right)^{\prime}(t)\right\|_{V_{2}^{*}}\left\|E_{2} z(t)\right\|_{V_{2}}+\left\|\left(E_{2} z\right)^{\prime}(t)\right\|_{V_{2}^{*}}\left\|E_{2} w(t)\right\|_{V_{2}} \\
& +2 \beta_{2}\left\|E_{2} w(t)\right\|_{V_{2}}\left\|E_{2} z(t)\right\|_{V_{2}} d t
\end{aligned}
$$

mit der Bezeichnung $\beta_{2}:=\|b\|_{\infty, \Omega_{2}}=\left(\sum_{i \in \underline{d}}\left\|b_{i}\right\|_{\infty, \Omega_{2}}^{2}\right)^{\frac{1}{2}}$. Mittels der Hölder-Ungleichung sowie Lemma 5.2.3 kann man weiter abschätzen

$$
\begin{gathered}
\int_{0}^{T}\left\|\left(E_{2} w\right)^{\prime}(t)\right\|_{V_{2}^{*}}\left\|E_{2} z(t)\right\|_{V_{2}}+\left\|\left(E_{2} z\right)^{\prime}(t)\right\|_{V_{2}^{*}}\left\|E_{2} w(t)\right\|_{V_{2}}+2 \beta_{2}\left\|E_{2} w(t)\right\|_{V_{2}}\left\|E_{2} z(t)\right\|_{V_{2}} d t \\
\leqslant\left(\int_{0}^{T}\left\|\left(E_{2} w\right)^{\prime}(t)\right\|_{V_{2}^{*}}^{2} d t\right)^{\frac{1}{2}}\left(\int_{0}^{T}\left\|E_{2} z(t)\right\|_{V_{2}}^{2} d t\right)^{\frac{1}{2}} \\
\quad+\left(\int_{0}^{T}\left\|\left(E_{2} z\right)^{\prime}(t)\right\|_{V_{2}^{*}}^{2} d t\right)^{\frac{1}{2}}\left(\int_{0}^{T}\left\|E_{2} w(t)\right\|_{V_{2}}^{2} d t\right)^{\frac{1}{2}} \\
\quad+2 \beta_{2}\left(\int_{0}^{T}\left\|E_{2} w(t)\right\|_{V_{2}}^{2} d t\right)^{\frac{1}{2}}\left(\int_{0}^{T}\left\|E_{2} z(t)\right\|_{V_{2}}^{2} d t\right)^{\frac{1}{2}} \\
\leqslant 2\left(1+\beta_{2}\right) C_{E_{2}}^{2}\|w\|_{Z}\|z\|_{Z} .
\end{gathered}
$$


Zu jedem $\gamma_{0}>0$ ergibt sich im Falle $\gamma \geqslant \gamma_{0}$ wegen der Koerzivität von $S_{2}^{(\gamma)}$ (siehe (6.18)) und der Stetigkeit von $S_{1}$ und $S_{2}($ siehe $(6.16))$ sowie wegen der Wahl $z=\left(S_{2}^{(\gamma)}\right)^{-1} S w$

$$
\begin{aligned}
\|z\|_{Z}^{2} & \leqslant \frac{1}{\gamma_{0}}\left\langle S_{2}^{(\gamma)} z, z\right\rangle=\frac{1}{\gamma_{0}}\langle S w, z\rangle \\
& \leqslant \frac{m_{1}+m_{2}}{\gamma_{0}}\|w\|_{Z}\|z\|_{Z},
\end{aligned}
$$

also

$$
\|z\|_{Z} \leqslant \frac{m_{1}+m_{2}}{\gamma_{0}}\|w\|_{Z}
$$

Damit folgt für $\gamma \geqslant \gamma_{0}>0$

$$
\left|\left\langle S_{2}^{(\gamma)} w, z\right\rangle-\left\langle S_{2}^{(\gamma)} z, w\right\rangle\right| \leqslant 2\left(1+\beta_{2}\right) C_{E_{2}}^{2} \frac{m_{1}+m_{2}}{\gamma_{0}}\|w\|_{Z}^{2}
$$

Ferner ist unter Annahme von (6.25)

$$
\langle S w, w\rangle \geqslant \alpha_{S}\|w\|_{Z}^{2}
$$

Mit $m_{S}:=m_{1}+m_{2}$, der Stetigkeitskonstante von $S$, gilt aufgrund der Ungleichung (6.27) daher insgesamt

$$
\left\langle S_{2}^{(\gamma)} w,\left(S_{2}^{(\gamma)}\right)^{-1} S w\right\rangle+\langle S w, w\rangle \geqslant 2\left(\alpha_{S}-\left(1+\beta_{2}\right) \frac{m_{S} C_{E_{2}}^{2}}{\gamma_{0}}\right)\|w\|_{Z}^{2}
$$

Wählt man nun ein positives $\gamma_{0}>\left(1+\beta_{2}\right) \frac{m_{S} C_{E_{2}}^{2}}{\alpha_{S}}$, so wird für jedes $\gamma \geqslant \gamma_{0}$ die abgeschwächte Koerzivitätsbedingung (6.24) durch

$$
\kappa=\kappa(\gamma):=2\left(\alpha_{S}-\left(1+\beta_{2}\right) \frac{m_{S} C_{E_{2}}^{2}}{\gamma_{0}}\right)
$$

erfüllt.

Zudem sind die Stetigkeitsvoraussetzungen (6.22) wegen (6.17) mit $l_{i}:=m_{i}+\|\gamma\|_{L^{\infty}(\Gamma)}$ und die Koerzivitätsvoraussetzung (6.23) wegen (6.18) mit $\mu_{2}:=\gamma_{0}$ erfüllt. Die Behauptung folgt daher mit

$$
\sigma^{*}:=\frac{\kappa(\gamma) \mu_{2}^{2}}{l_{2}\left(l_{1}+l_{2}\right)}
$$

und $Q_{i}=S_{i}^{(\gamma)}$ nach Satz 6.3.1.

Aus dem vorausgegangenen Beweis wird eine entscheidende Wirkung des Parameters $\gamma$ deutlich. Mit diesem kann die Störung der Symmetrie von $S_{2}^{(\gamma)}$ bezüglich zweier Elemente $w$ und 
$\left(S_{2}^{(\gamma)}\right)^{-1} S w$ hinreichend klein gehalten werden. In der Randbedingung $(5.14)_{2}$ wächst mit $\gamma$ der Dirichlet-Anteil.

Wir sind nun in der Lage, unser abschließendes Resultat zur Konvergenz der Lösung des Dirichlet-Robin-Algorithmus gegen die Lösung des Zweigebietsproblems zu zeigen, nach wie vor unter der Annahme (6.25).

Satz 6.3.3 Die Gültigkeit von (6.25) sei angenommen. Dann lassen sich Konstanten $\gamma_{0}>0$ und $\sigma^{*} \in(0,1]$ angeben, so daß die Lösung $\left(\left(u_{1}^{k}, u_{2}^{k}\right)\right)_{k \in \mathbb{N}}$ des Iterationsschemas (5.13) - (5.15) für jedes $\gamma \geqslant \gamma_{0}$ und zu beliebigem Initialwert $g^{0} \in Z$ gegen die Lösung $\left(u_{1}, u_{2}\right)$ des Problems (5.10) konvergiert, sofern der Relaxationsparameter $\sigma$ aus $\left(0, \sigma^{*}\right)$ ist.

Beweis. (i) Wähle $\gamma_{0}$ und $\sigma^{*}$ gemäß Lemma 6.3.2. Dann folgt aus diesem und mit (6.8) für jedes $\sigma \in\left(0, \sigma^{*}\right)$ die Existenz einer Konstante $C_{\sigma}<1$, so daß

$$
||\left|u_{i}^{k+1}\right|_{\Gamma}-\left.u_{i}\right|_{\Gamma}\left\||=||| K_{\sigma}\left(\left.u_{i}^{k}\right|_{\Gamma}-\left.u_{i}\right|_{\Gamma}\right)\left|\left\|\left|\leqslant C_{\sigma}\right|\right\| u_{i}^{k}\right|_{\Gamma}-\left.u_{i}\right|_{\Gamma}\right\| \mid \quad \text { für alle } k \in \mathbb{N}_{0}, i=1,2 .
$$

Induktiv ergibt sich daher für alle $k \in \mathbb{N}_{0}$ die Abschätzung

$$
||\left|u_{i}^{k}\right|_{\Gamma}-\left.u_{i}\right|_{\Gamma}\left|\left\|\leqslant C_{\sigma}^{k}\left|\left\|g^{0}-\left.u_{i}\right|_{\Gamma} \mid\right\|, \quad i=1,2\right.\right.\right.
$$

also die Konvergenz $\left(\left.u_{1}^{k}\right|_{\Gamma},\left.u_{2}^{k}\right|_{\Gamma}\right) \rightarrow\left(\left.u_{1}\right|_{\Gamma},\left.u_{1}\right|_{\Gamma}\right)$ bezüglich der Norm $\|\mid \cdot\| \cdot$

(ii) Die gewünschte Konvergenz von $\left(\left(u_{1}^{k}, u_{2}^{k}\right)\right)_{k \in \mathbb{N}}$ ergibt sich als Konsequenz. Wir benutzen wieder die Bezeichnung $\tilde{u}_{i}^{k}:=u_{i}^{k}-u_{i}, i=1,2$. Nach der Fehlergleichung (6.2) gilt

$$
\int_{0}^{T}\left\langle\left(\tilde{u}_{2}^{k}\right)^{\prime}(t), v(t)\right\rangle_{V_{2}^{*} \times V_{2}}+a_{2}\left(\tilde{u}_{2}^{k}(t), v(t)\right) d t=0 \quad \text { für alle } v \in H^{1}\left(0, T ; V_{2,0}\right) .
$$

Nach Definition des Operators $E_{2}$ erhält man daher $\tilde{u}_{2}^{k}=\left.E_{2} \tilde{u}_{2}^{k}\right|_{\Gamma}$ und folglich

$$
\begin{aligned}
\left.\left\|\left|\tilde{u}_{2}^{k}\right|_{\Gamma}\right\|\right|^{2} \geqslant & \int_{0}^{T} \frac{1}{2} \frac{d}{d t}\left\|\left.E_{2} \tilde{u}_{2}^{k}\right|_{\Gamma}(t)\right\|_{0}^{2}+a_{2}\left(\left.E_{2} \tilde{u}_{2}^{k}\right|_{\Gamma}(t),\left.E_{2} \tilde{u}_{2}^{k}\right|_{\Gamma}(t)\right) d t \\
& \quad+\gamma_{0}\left\|\left.\tilde{u}_{2}^{k}\right|_{\Gamma}\right\|_{L^{2}(0, T ; W)}^{2} \\
\geqslant & \int_{0}^{T} \alpha_{2}\left\|\left.E_{2} \tilde{u}_{2}^{k}\right|_{\Gamma}(t)\right\|_{1, \Omega_{2}}^{2} d t \\
= & \int_{0}^{T} \alpha_{2}\left\|\tilde{u}_{2}^{k}(t)\right\|_{1, \Omega_{2}}^{2} d t=\alpha_{2}\left\|\tilde{u}_{2}^{k}\right\|_{L^{2}\left(0, T ; V_{2}\right)}^{2}
\end{aligned}
$$

wegen $\gamma_{0}>0$ und der Koerzivität von $a_{2}$ (siehe (5.12)). 
Wie für die Differenz $\tilde{u}_{2}^{k}$ gilt nach (6.1) analog auch $\tilde{u}_{1}^{k}=\left.E_{1} \tilde{u}_{1}^{k}\right|_{\Gamma}$. Zusammen mit der Beziehung (6.2) liefert dies

$$
\begin{aligned}
\left\langle\left. S_{2}^{(\gamma)} \tilde{u}_{2}^{k}\right|_{\Gamma},\left.\tilde{u}_{1}^{k}\right|_{\Gamma}\right\rangle= & \int_{0}^{T}\left\langle\left(\left.E_{2} \tilde{u}_{2}^{k}\right|_{\Gamma}\right)^{\prime}(t),\left.E_{2} \tilde{u}_{1}^{k}\right|_{\Gamma}(t)\right\rangle_{V_{1}^{*} \times V_{1}} \\
& \quad+a_{2}\left(\left.E_{2} \tilde{u}_{2}^{k}\right|_{\Gamma}(t),\left.E_{2} \tilde{u}_{1}^{k}\right|_{\Gamma}(t)\right) d t+\left\langle\left.\gamma \tilde{u}_{2}^{k}\right|_{\Gamma},\left.\tilde{u}_{1}^{k}\right|_{\Gamma}\right\rangle_{Z} \\
= & \int_{0}^{T}\left\langle\left(\tilde{u}_{2}^{k}\right)^{\prime}(t),\left.E_{2} \tilde{u}_{1}^{k}\right|_{\Gamma}(t)\right\rangle_{V_{1}^{*} \times V_{1}} \\
& \quad+a_{2}\left(\tilde{u}_{2}^{k}(t),\left.E_{2} \tilde{u}_{1}^{k}\right|_{\Gamma}(t)\right) d t+\left\langle\left.\gamma \tilde{u}_{2}^{k}\right|_{\Gamma},\left.\tilde{u}_{1}^{k}\right|_{\Gamma}\right\rangle_{Z} \\
= & -\int_{0}^{T}\left\langle\left(\tilde{u}_{1}^{k}\right)^{\prime}(t),\left.E_{1} \tilde{u}_{1}^{k}\right|_{\Gamma}(t)\right\rangle_{V_{1}^{*} \times V_{1}} \\
& \quad+a_{1}\left(\tilde{u}_{1}^{k}(t),\left.E_{1} \tilde{u}_{1}^{k}\right|_{\Gamma}(t)\right) d t+\left\langle\left.\gamma \tilde{u}_{1}^{k}\right|_{\Gamma},\left.\tilde{u}_{1}^{k}\right|_{\Gamma}\right\rangle_{Z} \\
= & -\int_{0}^{T} \frac{1}{2} \frac{d}{d t}\left\|\left.\tilde{u}_{1}^{k}\right|_{\Gamma}(t)\right\|_{0}^{2}+a_{1}\left(\tilde{u}_{1}^{k}(t), \tilde{u}_{1}^{k}(t)\right) d t+\left\langle\left.\gamma \tilde{u}_{1}^{k}\right|_{\Gamma},\left.\tilde{u}_{1}^{k}\right|_{\Gamma}\right\rangle_{Z}
\end{aligned}
$$

Wegen der Koerzivität von $a_{1}$ sowie der Stetigkeit von $S_{2}^{(\gamma)}$ (siehe (5.12) bzw. (6.17)) ist folglich

$$
\begin{aligned}
\int_{0}^{T} \alpha_{1}\left\|\tilde{u}_{1}^{k}(t)\right\|_{1, \Omega_{1}}^{2} d t \leqslant & \int_{0}^{T} \frac{1}{2} \frac{d}{d t}\left\|\tilde{u}_{1}^{k}(t)\right\|_{0}^{2}+a_{1}\left(\tilde{u}_{1}^{k}(t), \tilde{u}_{1}^{k}(t)\right) d t \\
= & -\left\langle\left. S_{2}^{(\gamma)} \tilde{u}_{2}^{k}\right|_{\Gamma},\left.\tilde{u}_{1}^{k}\right|_{\Gamma}\right\rangle+\left\langle\left.\gamma \tilde{u}_{1}^{k}\right|_{\Gamma},\left.\tilde{u}_{1}^{k}\right|_{\Gamma}\right\rangle_{Z} \\
\leqslant & \left(\left(m_{2}+\|\gamma\|_{L^{\infty}(\Gamma)}\right)\left\|\left.\tilde{u}_{2}^{k}\right|_{\Gamma}\right\|_{Z}\right. \\
& \left.\quad+\|\gamma\|_{L^{\infty}(\Gamma)}\left\|\left.\tilde{u}_{1}^{k}\right|_{\Gamma}\right\|_{L^{2}(0, T ; W)}\right)\left\|\left.\tilde{u}_{1}^{k}\right|_{\Gamma}\right\|_{Z} \\
\leqslant & \frac{1}{\gamma_{0}}\left(\left(m_{2}+\|\gamma\|_{L^{\infty}(\Gamma)}\right)\left\|\left.\left.\tilde{u}\right|_{\Gamma} ^{k}\right|_{\Gamma}\right\|+\|\gamma\|_{L^{\infty}(\Gamma)}\left\|\left.\tilde{u}_{1}^{k}\right|_{\Gamma}\right\| \|\right)\left\|\left.\tilde{u}_{1}^{k}\right|_{\Gamma}\right\|,
\end{aligned}
$$

wobei für die letzte Abschätzung die Äquivalenz (6.20) ausgenutzt wurde.

Insgesamt schließt man aus (6.28) und (6.29) sowie $\left.|| \tilde{u}_{2}^{k}\right|_{\Gamma}|| \stackrel{k \rightarrow \infty}{\longrightarrow} 0$ und $\left\|\left.\tilde{u}_{1}^{k}\right|_{\Gamma}\right\| \stackrel{k \rightarrow \infty}{\longrightarrow} 0$ :

$$
\left(\left\|\tilde{u}_{1}^{k}\right\|_{L^{2}\left(0, T ; V_{1}\right)},\left\|\tilde{u}_{2}^{k}\right\|_{L^{2}\left(0, T ; V_{2}\right)}\right) \stackrel{k \rightarrow \infty}{\longrightarrow}(0,0),
$$

d. h. die Konvergenz $\left(u_{1}^{k}, u_{2}^{k}\right) \stackrel{k \rightarrow \infty}{\longrightarrow}\left(u_{1}, u_{2}\right)$ in $L^{2}\left(0, T ; V_{1}\right) \times L^{2}\left(0, T ; V_{2}\right)$.

Im Beweis zu Satz 6.3.3 tritt - unter Annahme von (6.25) - die lineare Konvergenzeigenschaft unseres Dirichlet-Robin-Algorithmus hervor. Im Abschnitt 7.4 werden wir diese Eigenschaft durch eine Reihe von numerischen Beispielen illustrieren.

Lemma 6.3.2 und dessen Beweis sind wegen der Unbestimmtheit der dort auftretenden Konstanten nicht geeignet, eine untere Schranke für den Parameter $\gamma$ tatsächlich anzugeben. Es 
handelt sich lediglich um Existenzaussagen. Die Konvergenzgeschwindigkeit hängt jedoch in der Regel sensibel von der Wahl des Parameters $\gamma$ ab. In der Praxis ist es daher nicht ausreichend, irgendein hinreichend großes $\gamma$ heranzuziehen. Vielmehr macht erst die Bestimmung eines im bestimmten Sinne optimalen oder zumindest annähernd optimalen $\gamma$ den Einsatz des Dirichlet-Robin-Algorithmus attraktiv. Untersuchungen dazu werden Gegenstand von Kapitel 8 sein. 


\section{Kapitel 7}

\section{Gebietszerlegung und Finite-Volumen-Diskretisierung}

Bisher haben wir Zweigebietsformulierungen und einen Dirichlet-RobinGebietszerlegungsalgorithmus für Konvektions-Diffusions-Reaktions-Probleme über unendlich-dimensionalen Sobolev-Räumen untersucht. In diesem Kapitel sollen diese Untersuchungen auf diskretem Level geführt werden als Voraussetzung für eine numerische Lösung der Probleme.

Zur Anwendung kommt dabei die Finite-Volumen-Technik, wie sie in Kapitel 2 und 4 entwickelt wurde. Ziel ist es, die Konvergenzuntersuchung des vorausgegangenen Kapitels auf den semidiskretisierten Fall zu übertragen.

\subsection{Finite-Volumen-Approximation}

Da wir unser Gebietszerlegungsverfahren für zeitkontinuierliche Probleme entwickelt haben, können wir uns bei der Diskretisierung an das im Abschnitt 1.2 skizzierte Vorgehen mittels Linienmethode halten. Das Ausgangsproblem wird zunächst räumlich diskretisiert und für das so entstandene semidiskrete Problem der Gebietszerlegungsalgorithmus formuliert. Auf diese Weise entstehen semidiskretisierte Probleme über jedem der Teilgebiete.

Es sei $\Omega \subseteq \mathbb{R}^{d}$ ein polyedrisches Lipschitz-Gebiet und $\mathcal{T}_{h}$ eine Triangulierung darauf, wie im Abschnitt 2.1 beschrieben, mit Knoten $\left\{a_{1}, \ldots, a_{N_{h}}\right\}$. Die Anzahl der inneren Knoten sei $N_{h, 0}$, und $\mathcal{B}_{h}^{D}=\left\{B_{1}, \ldots, B_{N_{h}}\right\}$ bezeichne das Donald-Diagramm zu $\mathcal{T}_{h}$.

Wir wiederholen die stabilisierte Finite-Volumen-Diskretisierung des Konvektions-DiffusionsReaktions-Problems (5.5) - (5.8):

Finde $u_{h} \in H^{1}\left((0, T) ; V_{h}\right), V_{h}=P_{0}^{1}\left(\mathcal{T}_{h}\right)$, so daß

$$
\left\{\begin{aligned}
\frac{d}{d t}\left(u_{h}(t), v\right)+a_{h}^{u p}\left(u_{h}(t), v\right) & =\left\langle F_{h}(t), v\right\rangle \quad \text { für fast alle } t \in(0, T) \text { und alle } v \in V_{h} \\
u_{h}(0) & =u_{0, h} \in V_{h} .
\end{aligned}\right.
$$


mit Bilinearform

$$
a_{h}(u, v)=\sum_{j \in N_{h, 0}}\left(-\varepsilon v_{j} \int_{\partial B_{j}} \nabla u \cdot d s+v_{j} \sum_{k \in I_{j}}\left(u_{j} \lambda_{j k}+\left(1-\lambda_{j k}\right) u_{k}\right) \beta_{j k}+\gamma_{j} u_{j} v_{j}\right)
$$

und Linearform

$$
\left\langle F_{h}(t), v\right\rangle=\sum_{j \in \underline{N_{h, 0}}} v_{j} \int_{B_{j}} f(t) d x
$$

für $u, v \in V_{h}$. Dabei ist $u_{j}=u\left(a_{j}\right), v_{j}\left(a_{j}\right), I_{i}:=\left\{j \in N_{h, 0} \backslash\{i\} \mid B_{i} \cap B_{j} \neq \emptyset\right\}$, und die Größen $\beta_{j k}, \gamma_{j}, \lambda_{j k}$ seien wie im Abschnitt 4.1 gewählt.

Darüber hinaus geben wir eine Zerlegung von $\Omega$ in Gebiete $\Omega_{1}, \Omega_{2}$ vor, die mit der Triangulierung $\mathcal{T}_{h}$ im folgenden Sinne verträglich ist. Für jedes $T \in \mathcal{T}_{h}$ und $i \in \underline{2}$ gilt

$$
T \cap \Omega_{i} \neq \emptyset \Longrightarrow T \subset \overline{\Omega_{i}} .
$$

Jedes Teilgebiet der Zerlegung ist also Vereinigung von Simplizes der Ausgangstriangulierung, insbesondere "zerschneidet" der Austauschrand $\Gamma:=\overline{\Omega_{1}} \cap \overline{\Omega_{2}}$ kein $T \in \mathcal{T}_{h}$. Auf beiden Teilgebieten $\Omega_{1}, \Omega_{2}$ induziert $\mathcal{T}_{h}$ somit Triangulierungen $\mathcal{T}_{h, 1}$ bzw. $\mathcal{T}_{h, 2}$, die ebenfalls den Bedingungen aus dem Abschnitt 2.1 genügen, sowie die zugehörigen Donald-Diagramme $\mathcal{B}_{h, 1}^{D}$ bzw. $\mathcal{B}_{h, 2}^{D}$. Abbildung 7.1 zeigt dies schematisch.

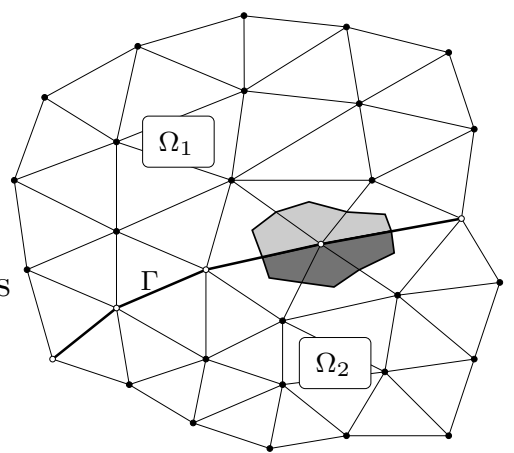

Abbildung 7.1: Zerlegung $\left\{\Omega_{1}, \Omega_{2}\right\}$ von $\Omega$ mit Triangulierung und beispielhaft dargestellten Kontrollvolumina

Mit $\left\{a_{1}^{(i)}, \ldots, a_{N_{i, h}}^{(i)}\right\}$ bezeichnen wir die Knoten der Triangulierung $\mathcal{T}_{h, i}, i \in \underline{2}$, die in $\Omega$ liegen, und mit $B_{j}^{(i)}$ das Volumen aus $\mathcal{B}_{h, i}^{D}$ zum Knoten $a_{j}^{(i)} . \mathcal{T}_{h, 1}$ und $\mathcal{T}_{h, 2}$ haben gerade die Knoten auf dem Austauschrand $\Gamma$ gemeinsam, deren Anzahl sei $N_{B, h}$, und es gelte $a_{j}^{(1)}=a_{j}^{(2)} \in \Gamma$ für $j \in N_{B, h}$.

Zur Formulierung der diskreten Probleme über den Teilgebieten nutzen wir die Räume

$$
V_{i, h}:=\left\{\left.v_{h}\right|_{\Omega_{i}} \mid v_{h} \in V_{h}\right\}, \quad V_{i, h}^{0}:=\left\{v_{h} \in V_{i, h}\left|v_{h}\right|_{\Gamma}=0\right\}
$$

und

$$
W_{h}:=\left\{\left.v_{h}\right|_{\Gamma} \mid v_{h} \in V_{h}\right\} .
$$


Wiederum können die globalen Funktionen aus $V_{h}$ mit Paaren $\left(u_{1, h}, u_{2, h}\right) \in V_{1, h} \times V_{2, h}$, für die $\left.u_{1, h}\right|_{\Gamma}=\left.u_{2, h}\right|_{\Gamma}$ gilt, identifiziert werden, und es ist $W_{h}=\operatorname{tr}_{\Gamma, 1}\left[V_{1, h}\right]=\operatorname{tr}_{\Gamma, 2}\left[V_{2, h}\right]$. Daneben bilden die Restriktionen $\left\{\left.\varphi_{j}\right|_{\overline{\Omega_{i}}} \mid a_{j} \in \overline{\Omega_{i}}\right\}$ der nodalen Basisfunktionen $\left\{\varphi_{1}, \ldots, \varphi_{N_{h}}\right\}$ eine Basis von $V_{i, h}, i=1,2$.

Wir leiten die Bilinearformen der stabilisierten Finiten-Volumen-Formulierung über den Teilgebieten aus den lokalen Bilinearformen (5.11) des kontinuierlichen Falls ab. Für diese gilt (siehe Bemerkung 5.2.2)

$$
a_{i}(u, v)=\int_{\Omega_{i}} \varepsilon \nabla v \cdot \nabla u+v \nabla \cdot(u b)+c u v d x-\frac{1}{2} \int_{\Gamma} u v b \cdot n_{i} d s, \quad u,\left.v \in H_{0}^{1}(\Omega)\right|_{\Omega_{i}} .
$$

Als entscheidend wird sich einmal mehr erweisen, daß zur Ableitung dieser lokalen Bilinearformen die Form (5.9) zugrunde gelegt wird, die in einen symmetrischen und einen schiefsymmetrischen Anteil aufspaltet, und nicht (5.6) (beide Formen unterscheiden sich über $V_{i}$ gerade durch den Randterm über dem Austauschrand $\Gamma$ ).

Die Bilinearform der entsprechenden Finiten-Volumen-Formulierung lautet (vergl. Abschnitt 2.3)

$$
\begin{aligned}
a_{i, h}(u, v):= & \sum_{B \in \mathcal{B}_{h, i}^{D}}\left(-\varepsilon \int_{\partial B \backslash \Gamma} \hat{v} \nabla u \cdot n_{i} d s+\int_{\partial B} u \hat{v} b \cdot n_{i} d s+\int_{B} c u \hat{v} d x\right) \\
& -\frac{1}{2} \int_{\Gamma} u \hat{v} b \cdot n_{i} d s \\
= & \sum_{j \in N_{i, h}}\left(-\varepsilon v_{j}^{(i)} \int_{\partial B_{j}^{(i)} \backslash \Gamma} \nabla u \cdot n_{i} d s+v_{j}^{(i)} \int_{\partial B_{j}^{(i)}} u b \cdot n_{i} d s+v_{j}^{(i)} \int_{B_{j}^{(i)}} c u d x\right) \\
& -\frac{1}{2} \sum_{j \in N_{B, h}} v_{j}^{(i)} \int_{\Gamma_{j}} u b \cdot n_{i} d s
\end{aligned}
$$

für Funktionen $u, v \in V_{i, h}$. Dabei ist $v_{j}^{(i)}:=v\left(a_{j}^{(i)}\right)$ und $\Gamma_{j}:=B_{j}^{(1)} \cap \Gamma=B_{j}^{(2)} \cap \Gamma$.

Analog zu den Bezeichnungen im Abschnitt 4.1 sei

$$
I_{j}^{(i)}:=\left\{k \in N_{i, h} \backslash\{j\} \mid B_{j}^{(i)} \cap B_{k}^{(i)} \neq \emptyset\right\}, \quad \Gamma_{j k}^{(i)}:=B_{j}^{(i)} \cap B_{k}^{(i)} .
$$

Wählen wir approximierende Größen $\beta_{j k}^{(i)}, \gamma_{j}^{(i)} \in \mathbb{R}$ wie in (4.5) und (4.6) bzw. (4.9), also $\beta_{j k}^{(i)}+\beta_{k j}^{(i)}=0$

$$
\left|\sum_{k \in I_{j}^{(i)}}\left(\int_{\Gamma_{j k}^{(i)}} b \cdot d s-\beta_{j k}^{(i)}\right)\right| \leqslant C h\left|B_{j}^{(i)}\right|, \quad\left|\int_{B_{j}^{(i)}} c d x-\gamma_{j}^{(i)}\right| \leqslant C h\left|B_{j}^{(i)}\right|, \quad j \in N_{i, h},
$$

sowie eine upwind-Funktion $\lambda: \mathbb{R} \rightarrow[0,1]$ gemäß $(\lambda 1)$ und $(\lambda 2)$, so erhalten wir schließlich 
die Bilinearform zur upwind-stabilisierten Diskretisierung über $\Omega_{i}$ :

$$
\begin{aligned}
a_{i, h}^{u p}(u, v):=\sum_{j \in N_{i, h}}( & -\varepsilon v_{j}^{(i)} \int_{\partial B_{j}^{(i)} \backslash \Gamma} \nabla u \cdot n_{i} d s+v_{j}^{(i)} \sum_{k \in I_{j}^{(i)}}\left(u_{j}^{(i)} \lambda_{j k}^{(i)}+\left(1-\lambda_{j k}^{(i)}\right) u_{k}^{(i)}\right) \beta_{j k}^{(i)} \\
& \left.+\gamma_{j}^{(i)} u_{j}^{(i)} v_{j}^{(i)}\right)+\frac{1}{2} \sum_{j \in N_{B, h}} u_{j}^{(i)} v_{j}^{(i)} \int_{\Gamma_{j}} b \cdot n_{i} d s
\end{aligned}
$$

mit $u_{j}^{(i)}:=u\left(a_{j}^{(i)}\right)$ und $\lambda_{i j}^{(i)}:=\lambda\left(\frac{\beta_{i j}^{(i)}}{\varepsilon}\right)$.

Die Bilinearform des diskreten globalen Problems (7.1) - (7.3) läßt sich in die Summe aus $a_{1, h}^{u p}$ und $a_{2, h}^{u p}$ zerlegen. Dazu setzen wir

$$
\beta_{j k}= \begin{cases}\beta_{l m}^{(i)} \operatorname{mit} a_{j}=a_{l}^{(i)}, a_{k}=a_{m}^{(i)}, & \text { falls } a_{j} \in \Omega_{i} \backslash \Gamma \text { oder } a_{k} \in \Omega_{i} \backslash \Gamma \\ \beta_{l m}^{(1)}+\beta_{l m}^{(2)} \text { mit } a_{j}=a_{l}^{(1)}=a_{l}^{(2)}, a_{k}=a_{m}^{(1)}=a_{m}^{(2)}, & \text { falls } a_{j}, a_{k} \in \Gamma\end{cases}
$$

und

$$
\gamma_{j}= \begin{cases}\gamma_{l}^{(i)} \quad \operatorname{mit} a_{j}=a_{l}^{(i)}, & \text { falls } a_{j} \in \Omega_{i} \backslash \Gamma \\ \gamma_{l}^{(1)}+\gamma_{l}^{(2)} \quad \operatorname{mit} a_{j}=a_{l}^{(1)}=a_{l}^{(2)}, & \text { falls } a_{j} \in \Gamma\end{cases}
$$

Man beachte dabei, daß $a_{j}, a_{k} \in \overline{\Omega_{i}}$ für ein $i \in \underline{2}$ gilt, sofern $a_{j}$ und $a_{k}$ benachbarte Knoten sind. Wegen (7.4) erfüllen $\beta_{j k}$ und $\gamma_{j}$ nach obiger Wahl (4.5) und (4.6) bzw. (4.9), und wegen $\int_{\Gamma_{j}} b \cdot n_{1} d s+\int_{\Gamma_{j}} b \cdot n_{2} d s=0$ gilt

$$
a_{h}^{u p}(u, v)=a_{1, h}^{u p}\left(\left.u\right|_{\overline{\Omega_{1}}},\left.v\right|_{\overline{\Omega_{1}}}\right)+a_{2, h}^{u p}\left(\left.u\right|_{\overline{\Omega_{2}}},\left.v\right|_{\overline{\Omega_{2}}}\right) .
$$

Mittels $a_{1, h}^{u p}$ und $a_{2, h}^{u p}$ können wir analog zum Abschnitt 5.2 eine zum globalen Problem gleichwertige Zweigebietsformulierung bezüglich der Zerlegung $\left\{\Omega_{1}, \Omega_{2}\right\}$ angeben:

Finde $\left(u_{1, h}, u_{2, h}\right) \in H^{1}\left(0, T ; V_{1, h}\right) \times H^{1}\left(0, T ; V_{2, h}\right)$ mit

$$
\left\{\begin{array}{l}
\frac{d}{d t}\left(u_{i, h}(t), v\right)_{\Omega_{i}}+a_{i, h}^{u p}\left(u_{i, h}(t), v\right)=(f(t), v)_{\Omega_{i}} \\
\text { für fast alle } t \in(0, T) \text { und alle } v \in V_{i, h}^{0}, \quad i=1,2 \\
\left.u_{1, h}(t)\right|_{\Gamma}=\left.u_{2, h}(t)\right|_{\Gamma} \quad \text { für fast alle } t \in(0, T) \\
\sum_{i \in \underline{2}}\left[\frac{d}{d t}\left(u_{i, h}(t), r_{i, h} w\right)_{\Omega_{i}}+a_{i, h}^{u p}\left(u_{i, h}(t), r_{i, h} w\right)\right]+\left\langle\left.\gamma u_{1, h}(t)\right|_{\Gamma}, w\right\rangle_{W}=\sum_{i \in \underline{2}}\left(f(t), r_{i, h} w\right)_{\Omega_{i}} \\
+\left\langle\left.\gamma u_{2, h}(t)\right|_{\Gamma}, w\right\rangle_{W} \quad \text { für fast alle } t \in(0, T) \text { und alle } w \in W \\
u_{i, h}(0)=\left.u_{0, h}\right|_{\Omega_{i}} \in L^{2}\left(\Omega_{i}\right), \quad i=1,2,
\end{array}\right.
$$


wobei $\gamma$ eine beliebige Funktion aus $L^{\infty}(\Gamma)$ und $\langle\cdot, \cdot\rangle_{W}$ das Skalarprodukt auf $W$ ist. Als Rechtsinverse $r_{i, h}$ zum Spuroperator $\operatorname{tr}_{\Gamma, i}: V_{i, h} \rightarrow W_{h}$ nutzen wir die Abbildung, die durch

$$
r_{i, h} w\left(a_{j}^{(i)}\right):= \begin{cases}w\left(a_{j}^{(i)}\right), & \text { falls } a_{j}^{(i)} \in \Gamma \\ 0 & \text { sonst }\end{cases}
$$

gegeben ist.

Lemma 7.1.1 Ist $u_{h} \in H^{1}\left(0, T ; V_{h}\right)$ eine Lösung von (7.1), so löst $\left(\left.u\right|_{\overline{\Omega_{1}}},\left.u\right|_{\Omega_{2}}\right)$ das Problem (7.5). Ist umgekehrt $\left(u_{1}, u_{2}\right)$ eine Lösung von (7.5), so ist die Funktion $u_{h} \in V_{h}$ mit

$$
u_{h}(t):= \begin{cases}u_{1, h}(t) & \text { in } \Omega_{1} \\ u_{2, h}(t) & \text { in } \Omega_{2}\end{cases}
$$

eine Lösung von (7.1).

Beweis. Der Beweis verläuft wie der Beweis von Lemma 5.1.2 unter Nutzung des diskreten Fortsetzungsoperators $r_{i, h}$ wie oben angegeben.

\subsection{Der Dirichlet-Robin-Algorithmus für das semidiskretisier- te Problem}

Wie für den kontinuierlichen Fall im Abschnitt 5.2 wird auf einem Teilgebiet die Randbedingung (7.5) 3 , auf dem anderen Teilgebiet die Randbedingung $(7.5)_{2}$ fallengelassen, um ein iteratives Gebietszerlegungsverfahren zu formulieren.

Der Dirichlet-Robin-Algorithmus zum Parameter $\gamma \in L^{\infty}(\Gamma)$ nach Finite-VolumenDiskretisierung lautet:

$\mathrm{Zu}$ einem Initialwert $g^{0} \in Z_{h}$ finde eine Folge von Funktionen $\left(u_{1, h}^{k}, u_{2, h}^{k}\right) \in H^{1}\left(0, T ; V_{1, h}\right) \times$ $H^{1}\left(0, T ; V_{2, h}\right)$ mit

$$
\left\{\begin{array}{c}
\frac{d}{d t}\left(u_{1, h}^{k}(t), v\right)_{\Omega_{1}}+a_{1, h}^{u p}\left(u_{1, h}^{k}(t), v\right)=\left\langle F_{1, h}(t), v\right\rangle \\
\text { für fast alle } t \in(0, T) \text { und alle } v \in V_{1, h}^{0} \\
\left.u_{1, h}^{k}(t)\right|_{\Gamma}=g^{k-1}(t) \quad \text { für fast alle } t \in(0, T) \\
u_{1, h}(0)=\left.u_{0, h}\right|_{\Omega_{1}},
\end{array}\right.
$$




$$
\begin{aligned}
& \int \frac{d}{d t}\left(u_{2, h}^{k}(t), v\right)_{\Omega_{2}}+a_{2, h}^{u p}\left(u_{2, h}^{k}(t), v\right)=(f(t), \hat{v})_{\Omega_{2}} \\
& \text { für fast alle } t \in(0, T) \text { und alle } v \in V_{2, h}^{0} \\
& \left\{\begin{array}{c}
\frac{d}{d t}\left(u_{2, h}^{k}(t), r_{2, h} w\right)_{\Omega_{2}}+a_{2, h}^{u p}\left(u_{2, h}^{k}(t), r_{2, h} w\right)+\left\langle\left.\gamma u_{2, h}^{k}(t)\right|_{\Gamma}, w\right\rangle_{W}=\left\langle F_{2, h}(t), r_{2, h} w\right\rangle \\
+\left\langle F_{1, h}(t), r_{1, h} w\right\rangle-\frac{d}{d t}\left(u_{1, h}^{k}(t), r_{1, h} w\right)_{\Omega_{1}}-a_{1, h}^{u p}\left(u_{1, h}^{k}(t), r_{1, h} w\right) \\
+\left\langle\left.\gamma u_{1, h}^{k}(t)\right|_{\Gamma}, w\right\rangle_{W} \quad \text { für fast alle } t \in(0, T) \text { und alle } w \in W_{h}
\end{array}\right. \\
& u_{2, h}(0)=\left.u_{0, h}\right|_{\Omega_{2}}
\end{aligned}
$$

für jedes $k \geqslant 1$. Dabei ist

$$
\left\langle F_{i, h}(t), v\right\rangle:=\int_{\Omega_{i}} f(t) \hat{v} d x
$$

und

$$
g^{k}:=\left.\sigma u_{2, h}^{k}\right|_{\Gamma}+(1-\sigma) g^{k-1}
$$

mit einem Relaxationsparameter $\sigma \in(0,1]$.

Dabei nutzen wir den Spurraum

$$
Z_{h}:=L^{2}\left(0, T ; W_{h}\right) \cap H^{\frac{1}{4}}\left(0, T ; L^{2}(\Gamma)\right)
$$

(vergl. A.3).

Um dem Vorgehen in Kapitel 6 folgen zu können, setzen wir (zumindest für hinreichend kleine $h$ ) die Existenz diskreter Fortsetzungsoperatoren $E_{i, h}: Z_{h} \rightarrow H^{1}\left(0, T ; V_{i, h}\right), i=1$, 2 , voraus, die sich analog zu (5.16) über

$$
\left\{\begin{array}{l}
\frac{d}{d t}\left(E_{i, h} w(t), v\right)_{\Omega_{i}}+a_{i, h}^{u p}\left(E_{i, h} w(t), v\right)=0 \quad \text { für fast alle } t \in(0, T) \\
\left.E_{i, h} w(t)\right|_{\Gamma}=w(t) \quad \text { fast alle } t \in(0, T) \text { und alle } v \in V_{i, 0} \\
E_{i, h} w(0)=0
\end{array}\right.
$$

definieren und die den Aussagen aus Lemma 5.2.3 genügen:

$$
\left\|E_{i, h} w\right\|_{H^{1}\left(0, T ; V_{i}\right)} \leqslant C_{E_{i, h}}\|w\|_{Z}, \quad\|w\|_{Z} \leqslant C_{\Omega_{i}}\left\|E_{i, h} w\right\|_{H^{1}\left(0, T ; V_{i}\right)}, \quad w \in Z_{h},
$$

mit einer von $\varepsilon$ abhängigen Konstante $C_{E_{i, h}}=C_{E_{i, h}}(\varepsilon)>0$. An dieser Stelle müssen wir den Beweis der angenommenen Existenz von $E_{i, h}$ offen lassen. Die Existenz entsprechender diskreter Fortsetzungsoperatoren im elliptischen Fall (siehe [11]) legt jedoch die Sinnhaftigkeit der Annahme nahe. Im vorliegenden zeitabhängigen Fall tritt die Forderung der zeitlichen Regularität hinzu und ist zu berücksichtigen. 
Den Nachweis, daß die lokalen Bilinearformen $a_{i, h}^{u p}, i=1,2$, die benötigten Eigenschaften Koerzivität und Stetigkeit tragen, führen wir im nächsten Lemma und gehen dabei wie in den Abschnitten 4.2 und 4.3 vor. Zur Nutzung der Konsistenzfehlerabschätzungen aus den Abschnitten 3.1 und 4.3 setzen wir

$$
b \in\left(H^{1, \infty}(\Omega)\right)^{2}, \quad c \in H^{1, \infty}(\Omega)
$$

voraus. Die Wohlgestelltheit der lokalen Probleme ergibt sich dann durch den

Satz 7.2.1 Es gibt ein $h_{0}>0$, so daß für $h \in\left(0, h_{0}\right)$ die beiden Teilprobleme (7.6) und (7.7) in jedem Iterationsschritt $k \in \mathbb{N}$ eine eindeutige Lösung in $H^{1}\left(0, T ; V_{1, h}\right)$ bzw. $H^{1}\left(0, T ; V_{2, h}\right)$ besitzen, falls $\gamma \geqslant \gamma_{0}>0$.

Beweis. $\quad$ Wir ziehen Lemma 3.1.1 und 3.1.2 sowie aufgrund der Voraussetzung (7.11) Lemma 4.3.1 und Bemerkung 3.1.4 heran und erhalten

$$
\begin{aligned}
\left|a_{i}(u, v)-a_{i, h}^{u p}(u, v)\right| & \leqslant\left|a_{i}(u, v)-a_{i, h}(u, v)\right|+\left|a_{i, h}(u, v)-a_{i, h}^{u p}(u, v)\right| \\
& \leqslant h C_{\infty, i}\|u\|_{1, \Omega_{i}}\|v\|_{1, \Omega_{i}},
\end{aligned}
$$

$u, v \in V_{i, h}$, mit $C_{\infty, i}:=C\left(1+\|b\|_{1, \infty, \Omega_{i}}+\|c\|_{1, \infty, \Omega_{i}}+\left\|\left.b\right|_{\Gamma}\right\|_{1, \infty, \Gamma}\right), i=1,2$, und einer einer sowohl von $h$ als auch von Daten des Problems (5.5) - (5.8) unabhängigen Konstante $C>0$. Die Stetigkeit von $a_{i, h}^{u p}$ ergibt sich daher infolge von (5.12) und (7.12):

$$
a_{i, h}^{u p}(u, v) \leqslant\left(M_{i}+h C_{\infty, i}\right)\|u\|_{1, \Omega_{i}}\|v\|_{1, \Omega_{i}} \quad \text { für alle } u, v \in V_{i, h}
$$

$\operatorname{mit} M_{i}=M_{\Omega_{i}}=\varepsilon+\|q\|_{\infty, \Omega_{i}}+\|b\|_{\infty, \Omega_{i}}, i=1,2$.

Um die Koerzivität von $a_{i, h}^{u p}$ zu zeigen, folgert man mit Lemma 3.1.1 und 4.1.1 sowie mit

$$
\int_{B_{j}^{(i)}} \nabla \cdot b d x=\sum_{k \in I_{j}^{(i)}} \int_{\Gamma_{j k}^{(i)}} b \cdot n_{i} d s+\int_{\Gamma_{j}} b \cdot n_{i} d s
$$

$$
\begin{aligned}
&\left(\int_{\Gamma_{j}} b \cdot n_{i} d s=\right.\left.0 \text { für } j>N_{B, h}\right) \\
& a_{i, h}^{u p}(v, v) \geqslant \varepsilon|v|_{1, \Omega_{i}}^{2}+\frac{1}{2} \sum_{j \in \underline{N_{i, h}}} \sum_{k \in I_{j}^{(i)}}\left(v_{j}^{(i)}\right)^{2} \beta_{j k}^{(i)}+\frac{1}{2} \sum_{j \in N_{B, h}}\left(v_{j}^{(i)}\right)^{2} \int_{\Gamma_{j}} b \cdot n_{i} d s+\sum_{j \in N_{i, h}} \gamma_{j}\left(v_{j}^{(i)}\right)^{2} \\
&=\varepsilon|v|_{1, \Omega_{i}}^{2}+\sum_{j \in N_{i, h}}\left(v_{j}^{(i)}\right)^{2}\left[\int_{B_{j}^{(i)}} \frac{1}{2} \nabla \cdot b+c d x-\frac{1}{2} \sum_{k \in I_{j}^{(i)}}\left(\int_{\Gamma_{j k}^{(i)}} b \cdot d s-\beta_{j k}^{(i)}\right)\right. \\
&\left.-\int_{B_{j}^{(i)}} c d x+\gamma_{j}^{(i)}\right]
\end{aligned}
$$

für alle $v \in V_{i, h}$. Völlig analog zum Beweis von Satz 4.2.2 ergibt sich dann wegen (5.8), (7.4) und Lemma 4.2.1 mit einer von $h$ unabhängigen Konstante $C_{i, 0}>0$

$$
a_{i, h}^{u p}(v, v) \geqslant \varepsilon|v|_{1, \Omega_{i}}^{2}+(\eta-C h) \sum_{j \in \underline{N_{i, h}}}\left(v_{j}^{(i)}\right)^{2}\left|B_{j}^{(i)}\right| \geqslant \varepsilon|v|_{1, \Omega_{i}}^{2}+\frac{1}{2} \eta C_{i, 0}\|v\|_{0, \Omega_{i}}^{2}
$$


für $h \leqslant h_{0}=\frac{\eta}{2 C}$. Man beachte, daß gegenüber Satz 4.2.2 hier Randterme über dem Austauschrand $\Gamma$ zu berücksichtigen sind und daß in diesem Kapitel generell die Numerierung der Knoten abweichend ist von der in Kapitel 4.

Schließlich erhält man

$$
a_{i, h}^{u p}(v, v) \geqslant \alpha_{u p, i}\|v\|_{1, \Omega_{i}}, \quad v \in V_{i, h},
$$

$\operatorname{mit} \alpha_{u p, i}:=\min \left\{\varepsilon, \frac{1}{2} \eta C_{0}\right\}, i=1,2$.

Damit läßt sich der Beweis wie der Beweis zu Satz 5.2.4 zu Ende führen.

Hier und im weiteren Verlauf des Kapitels werden Aussagen unter der Bedingung gemacht, daß $h$ hinreichend klein ist. Die obere Schranke $h_{0}$ geht dabei auf die Approximationen (7.4) zurück und hängt nur von der dort auftretenden Konstante $C$ sowie von $\eta$ ab. Verwendet man für $\beta_{j k}^{(i)}$ und $\gamma_{j}^{(i)}$ jeweils die exakten Integrale, so gelten die entsprechenden Aussagen ohne Beschränkung an $h$ (vergl. Bemerkung 4.2.3).

\subsection{Konvergenzanalyse im semidiskretisierten Fall}

Die Aussagen, die in Kapitel 6 zur Konvergenzanalyse des Dirichlet-Robin-Algorithmus im kontinuierlichen Fall gemacht wurden, hingen von der Strukur des Gebietszerlegungsalgorithmus, der Wahl der Austauschrandfunktion $\gamma$ sowie den Eigenschaften der dort auftretenden lokalen Bilinearformen, nicht jedoch - bis auf eine Ausnahme - von der konkreten Gestalt der Bilinearformen ab.

Da sich die wesentlichen Eigenschaften, Koerzivität und Stetigkeit, auf $a_{1, h}^{u p}$ und $a_{2, h}^{u p}$ zumindest für hinreichend kleine $h$ übertragen ließen, kann das Vorgehen in Kapitel 6 direkt auf den semidiskreten Fall dieses Kapitels übertragen werden. Wir zeigen die einzelnen Schritte auf, ohne erneut alle Details auszuführen. Die oben erwähnte Ausnahme tritt im Beweis von Lemma 7.3.2 auf, auch dort kann man letztlich auf den kontinuierlichen Fall zurückgreifen.

Wie im kontinuierlichen Fall betrachtet man zu einer Lösung $\left(\left(u_{1, h}^{k}, u_{2, h}^{k}\right)\right)_{k \in \mathbb{N}}$ von (7.6) (7.9) und einer Lösung $\left(u_{1, h}, u_{2, h}\right)$ von (7.5) mit identischen Anfangswerten die Differenzenfolge $\left(\left(\tilde{u}_{1, h}^{k}, \tilde{u}_{2, h}^{k}\right)\right)_{k \in \mathbb{N}}$ mit $\tilde{u}_{i, h}^{k}:=u_{i, h}^{k}-u_{i, h}, i=1,2$, und erhält die diskreten Fehlergleichungen

$$
\frac{d}{d t}\left(\tilde{u}_{1, h}^{k}(t), v\right)_{\Omega_{1}}+a_{1, h}^{u p}\left(\tilde{u}_{1, h}^{k}(t), v\right)=0 \quad \text { für alle } v \in V_{1, h}^{0}
$$

und

$$
\begin{gathered}
\frac{d}{d t}\left(\tilde{u}_{2, h}^{k}(t), v\right)_{\Omega_{2}}+a_{2, h}^{u p}\left(\tilde{u}_{2, h}^{k}(t), v\right)+\left\langle\left.\gamma \tilde{u}_{2, h}^{k}(t)\right|_{\Gamma},\left.v\right|_{\Gamma}\right\rangle_{W} \\
-a_{1, h}\left(\tilde{u}_{1, h}^{k}(t),\left.r_{1, h} v\right|_{\Gamma}\right)+\left\langle\left.\gamma \tilde{u}_{1, h}^{k}(t)\right|_{\Gamma},\left.v\right|_{\Gamma}\right\rangle_{W}
\end{gathered}
$$

Auch zu den Operatoren $K$ und $K_{\sigma}$ laut Abschnitt 6.1 lassen sich (wiederum für hinreichend kleine $h$ ) diskrete Entsprechungen $K_{h}$ bzw. $K_{h, \sigma}$ angeben, und Lemma 6.1.1 gilt übertragungsgemäß. 
Die Steklov-Poincaré-Operatoren haben hier die Gestalt

$$
\begin{aligned}
\left\langle S_{i, h} v, w\right\rangle & :=\int_{0}^{T}\left\langle\left(E_{i, h} v\right)^{\prime}(t), E_{i, h} w(t)\right\rangle_{V_{i, h}^{*} \times V_{i, h}}+a_{i, h}^{u p}\left(E_{i, h} v(t), E_{i, h} w(t)\right) d t, \quad i=1,2, \\
\left\langle S_{1, h}^{(\gamma)} v, w\right\rangle & :=\left\langle S_{1, h} v, w\right\rangle-\langle v, w\rangle_{Z} \\
\left\langle S_{2, h}^{(\gamma)} v, w\right\rangle & :=\left\langle S_{2, h} v, w\right\rangle+\langle v, w\rangle_{Z} .
\end{aligned}
$$

Zudem sei $S_{h}:=S_{1, h}+S_{2, h}$.

Mit der Koerzivität von $a_{i, h}^{u p},(7.14)$, und dem Spursatz A.2.3 folgt (vergl. (7.17))

$$
\begin{aligned}
\left\langle S_{i, h} w, w\right\rangle & =\int_{0}^{T} \frac{1}{2} \frac{d}{d t}\left\|E_{i, h} w(t)\right\|_{0, \Omega_{i}}^{2}+a_{i, h}^{u p}\left(E_{i, h} w(t), E_{i, h} w(t)\right) d t \\
& \geqslant\left\|E_{i, h} w(T)\right\|_{0, \Omega_{i}}^{2}+\alpha_{u p, i} C_{t r, i} \int_{0}^{T}\|w\|_{W}^{2} d t, \quad i=1,2
\end{aligned}
$$

Als semidiskrete Entsprechung zu Lemma 6.2.1 ergibt sich

Lemma 7.3.1 Es gibt ein $h_{0}>0$, so daß $S_{1, h}^{(\gamma)}$ und $S_{2, h}^{(\gamma)}$ lineare, stetige Abbildungen von $Z_{h}$ nach $Z_{h}^{*}$ sind für alle $h \in\left(0, h_{0}\right)$. Zudem ist $S_{2, h}^{(\gamma)}$ koerziv, sofern $\gamma \geqslant \gamma_{0}>0$ und $h \in\left(0, h_{0}\right)$.

Beweis. $\quad$ Für $v, w \in L^{2}(0, T ; W)$ gilt wegen $(7.13)$

$$
\left|\left\langle S_{i, h} v, w\right\rangle\right| \leqslant \int_{0}^{T}\left\|\left(E_{i, h} v\right)^{\prime}(t)\right\|_{V_{i}^{*}}\left\|E_{i, h} w(t)\right\|_{V_{i}}+\left(M_{i}+h C_{\infty, i}\right)\left\|E_{i, h} v(t)\right\|_{V_{i}}\left\|E_{i, h} w(t)\right\|_{V_{i}} d t .
$$

Daraus schließt man für hinreichend kleine $h$ wie im Beweis von Lemma 6.2.1 die Existenz der Steklov-Poincaré-Operatoren sowie vermöge (7.10) die Stetigkeitsaussagen

$$
\left|\left\langle S_{i, h} v, w\right\rangle\right| \leqslant m_{i, h}\|v\|_{Z}\|w\|_{Z}
$$

und

$$
\left|\left\langle S_{i, h}^{(\gamma)} v, w\right\rangle\right| \leqslant\left(m_{i, h}+\|\gamma\|_{L^{\infty}(\Gamma)}\right)\|v\|_{Z}\|w\|_{Z}
$$

mit $m_{i, h}:=\left(1+M_{i}+h C_{\infty, i}\right) C_{E_{i, h}}^{2}$ für $i=1,2$.

Wegen $\left\langle S_{2, h} w, w\right\rangle \geqslant 0$ für jedes $w \in Z_{h}$ gemäß (7.17) folgt schließlich

$$
\left\langle S_{2, h}^{(\gamma)} w, w\right\rangle \geqslant \gamma_{0}\|w\|_{Z}^{2}
$$

also die Koerzitivität von $S_{2, h}^{(\gamma)}$, für $\gamma \geqslant \gamma_{0}>0$.

Wir erhalten also analog zu Abschnitt 6.2 mit Hilfe von $S_{2, h}^{(\gamma)}$ ein Skalarprodukt - in diesem Fall auf dem räumlich diskreten Raum $Z_{h}$ : 


$$
\langle v, w\rangle_{2}:=\frac{1}{2}\left(\left\langle S_{2, h}^{(\gamma)} v, w\right\rangle+\left\langle S_{2, h}^{(\gamma)} w, v\right\rangle\right)
$$

Dieses induziert die $h$-abhängige Norm

$$
\|w\|_{h}:=\sqrt{\left\langle S_{2, h}^{(\gamma)} w, w\right\rangle}, \quad w \in L^{2}\left(0, T ; W_{h}\right),
$$

die der Abschätzung

$$
\sqrt{\gamma_{0}}\|w\|_{Z} \leqslant\|w\|_{h} \leqslant \sqrt{m_{i, h}+\|\gamma\|_{L^{\infty}(\Gamma)}}\|w\|_{Z} .
$$

genügt.

Wie in Lemma 6.2.2 erhält man die Darstellung

$$
K_{h, \sigma}=1-\sigma\left(S_{2, h}^{(\gamma)}\right)^{-1}\left(S_{1, h}+S_{2, h}\right)
$$

und schafft damit die Voraussetzung, ebenfalls unter Anwendung von Satz 6.3.1 die Kontraktionseigenschaft des Operators $K_{h, \sigma}$ zu zeigen. Dabei sind wir entsprechend zu (6.25) auch hier auf die Annahme angewiesen, daß die Summe der Steklov-Poincaré-Operatoren koerziv ist, es also ein $\omega>0$ gibt mit

$$
\left\langle S_{h} w, w\right\rangle=\int_{0}^{T}\left\langle\left(E_{i, h} w\right)^{\prime}(t), E_{i, h} w(t)\right\rangle_{V_{i, h}^{*} \times V_{i, h}}+a_{i, h}^{u p}\left(E_{i, h} w(t), E_{i, h} w(t)\right) d t \geqslant w\|w\|_{Z}^{2}
$$

Lemma 7.3.2 Die Gültigkeit von (7.23) sei angenommen. Mit der Beschränkung an $h$ aus Satz 7.2.1 lassen sich dann Konstanten $\gamma_{0}>0$ und $\sigma^{*} \in(0,1]$ angeben, so daß zu jedem $\sigma \in\left(0, \sigma^{*}\right)$ eine Konstante $C_{\sigma}<1$ existiert, für die

$$
\left\|K_{h, \sigma} w\right\|_{h} \leqslant C_{\sigma}\|w\|_{h} \quad \text { für alle } w \in L^{2}\left(0, T ; W_{h}\right)
$$

gilt, sofern $\gamma \in L^{\infty}(\Gamma)$ und $\gamma \geqslant \gamma_{0}$. Dabei ist $\|\cdot\|_{h}$ die durch $\gamma$ parametrisierte Norm (7.21).

Beweis. $\quad$ Für die Abweichung des Operators $\left\langle S_{2, h}^{(\gamma)}, \cdot\right\rangle$ von der Symmetrie ergibt sich in diesem Fall

$$
\begin{aligned}
\left|\left\langle S_{2, h}^{(\gamma)} w, z\right\rangle-\left\langle S_{2, h}^{(\gamma)} z, w\right\rangle\right|= & \mid \int_{0}^{T}\left\langle\left(E_{2, h} w\right)^{\prime}(t), E_{2, h} z(t)\right\rangle_{V_{2}^{*} \times V_{2}}+a_{2, h}^{u p}\left(E_{2, h} w(t), E_{2, h} z(t)\right) \\
& \quad-\left\langle\left(E_{2, h} z\right)^{\prime}(t), E_{2, h} w(t)\right\rangle_{V_{2}^{*} \times V_{2}}-a_{2, h}^{u p}\left(E_{2, h} z(t), E_{2, h} w(t)\right) d t \mid \\
\leqslant & \mid \int_{0}^{T}\left\langle\left(E_{2, h} w\right)^{\prime}(t), E_{2, h} z(t)\right\rangle_{V_{2}^{*} \times V_{2}}+a_{2}\left(E_{2, h} w(t), E_{2, h} z(t)\right) \\
& -\left\langle\left(E_{2, h} z\right)^{\prime}(t), E_{2, h} w(t)\right\rangle_{V_{2}^{*} \times V_{2}}-a_{2}\left(E_{2, h} z(t), E_{2, h} w(t)\right) d t \mid \\
& +\int_{0}^{T}\left|a_{2}\left(E_{2, h} z(t), E_{2, h} w(t)\right)-a_{2, h}^{u p}\left(E_{2, h} z(t), E_{2, h} w(t)\right)\right| d t .
\end{aligned}
$$


Mit Hilfe der Konsistenzfehlerabschätzung (7.12) und $\beta_{2}:=\|b\|_{\infty, \Omega_{2}}$ erhält man daher analog zum Beweis von Lemma 6.3.2

$$
\begin{aligned}
& \left\langle S_{2, h}^{(\gamma)} w,\left(S_{2, h}^{(\gamma)}\right)^{-1} S_{h} w\right\rangle+\left\langle S_{h} w, w\right\rangle \\
& \quad \geqslant 2\left\langle S_{h} w, w\right\rangle-2\left(1+\beta_{2}+h C_{\infty, 2}\right) C_{E_{2, h}}^{2}\|w\|_{Z}\left\|\left(S_{2, h}^{(\gamma)}\right)^{-1} S_{h} w\right\|_{Z}
\end{aligned}
$$

$\mathrm{Zu}$ jedem $\gamma_{0}>0$ ergibt sich im Falle $\gamma \geqslant \gamma_{0}$ wegen der Koerzivität von $S_{2}^{(\gamma)}($ siehe $(7.20))$ und der Stetigkeit von $S_{1}$ und $S_{2}$ (siehe (7.18))

$$
\left\|\left(S_{2, h}^{(\gamma)}\right)^{-1} S_{h} w\right\|_{Z} \leqslant \frac{m_{S_{h}}}{\gamma_{0}}\|w\|_{Z}
$$

mit $m_{S_{h}}:=m_{1, h}+m_{2, h}$. Daneben folgt unter Annahme von (7.23)

$$
\left\langle S_{h} w, w\right\rangle \geqslant \omega\|w\|_{Z}^{2}
$$

Insgesamt gilt

$$
\left\langle S_{2, h}^{(\gamma)} w,\left(S_{2, h}^{(\gamma)}\right)^{-1} S_{h} w\right\rangle+\left\langle S_{h} w, w\right\rangle \geqslant 2\left(\omega^{*}-\left(1+\beta_{2}+h C_{\infty, 2}\right) \frac{m_{S_{h}} C_{E_{2, h}}^{2}}{\gamma_{0}}\right)\|w\|_{Z}^{2}
$$

für $\gamma \geqslant \gamma_{0}>0$. Die Behauptung folgt nunmehr mit der Wahl eines

$$
\gamma_{0}>\max \left\{0,\left(1+\beta_{2}+h C_{\infty, 2}\right) \frac{m_{S_{h}} C_{E_{2, h}}^{2}}{\left.\omega_{2}^{*}\right\}}\right.
$$

wie im Beweis zu Lemma 6.3.2 unter Anwendung von Satz 6.3.1.

Schließlich können wir das Resultat aus Satz 6.3.3 übertragen und so mit der Annahme (7.23) die Konvergenz der Lösung des Dirichlet-Robin-Algorithmus gegen die Lösung des Zweigebietsproblems im Fall einer Finite-Volumen-Diskretisierung zeigen.

Satz 7.3.3 Die Gültigkeit von (7.23) sei angenommen. Mit der Beschränkung an $h$ aus Satz 7.2.1 lassen sich dann Konstanten $\gamma_{0}>0$ und $\sigma^{*} \in(0,1]$ angeben, so daß die Lösung $\left(\left(u_{1, h}^{k}, u_{2, h}^{k}\right)\right)_{k \in \mathbb{N}}$ des Iterationsschemas (7.6) - (7.9) für jedes $\gamma \geqslant \gamma_{0}$ und zu beliebigem Initialwert $g^{0} \in Z_{h}$ gegen die Lösung $\left(u_{1, h}, u_{2, h}\right)$ des Problems (7.5) konvergiert, sofern der Relaxationsparameter $\sigma$ aus $\left(0, \sigma^{*}\right)$ ist.

Beweis. Völlig analog zum Beweis von Satz 6.3.3 ergibt sich mit Lemma 7.3.2 für jedes $\sigma \in\left(0, \sigma^{*}\right)$ die Existenz einer Konstante $C_{\sigma}<1$, so daß

$$
||\left|u_{i, h}^{k+1}\right|_{\Gamma}-\left.\left.u_{i, h}\right|_{\Gamma}||\right|_{h}=||\left|K_{\sigma}\left(\left.u_{i, h}^{k}\right|_{\Gamma}-\left.u_{i, h}\right|_{\Gamma}\right)\right|\left\|_{h} \leqslant C_{\sigma}\right\|\left|u_{i, h}^{k}\right|_{\Gamma}-\left.u_{i, h}\right|_{\Gamma} \|_{h} \quad \text { für alle } k \in \mathbb{N}_{0} .
$$

Ebenfalls völlig analog erhält man $\tilde{u}_{i, h}^{k}=\left.E_{i, h} \tilde{u}_{i, h}^{k}\right|_{\Gamma}$ aufgrund der Fehlergleichungen (7.15) und (7.16). Folglich ist

$$
\begin{aligned}
\left\|\left.\tilde{u}_{2, h}^{k}\right|_{\Gamma}\right\| \|^{2} \geqslant & \int_{0}^{T} \frac{1}{2} \frac{d}{d t}\left\|\left.E_{2, h} \tilde{u}_{2, h}^{k}\right|_{\Gamma}(t)\right\|_{0}^{2}+a_{2, h}^{u p}\left(\left.E_{2, h} \tilde{u}_{2, h}^{k}\right|_{\Gamma}(t),\left.E_{2, h} \tilde{u}_{2, h}^{k}\right|_{\Gamma}(t)\right) d t \\
& \quad+\gamma_{0}\left\|\left.\tilde{u}_{2, h}^{k}\right|_{\Gamma}\right\|_{Z}^{2} \\
\geqslant & \alpha_{u p, 2}\left\|\tilde{u}_{2, h}^{k}\right\|_{L^{2}\left(0, T ; V_{2}\right)}^{2}
\end{aligned}
$$


wegen der Koerzivität von $a_{2, h}^{u p}($ siehe $(7.14))$ und

$$
\alpha_{u p, 1}\left\|\tilde{u}_{1, h}^{k}\right\|_{L^{2}\left(0, T ; V_{1}\right)}^{2} \leqslant\left(\left(m_{2, h}+\|\gamma\|_{L^{\infty}(\Gamma)}\right)\left\|\left.\tilde{u}_{2, h}^{k}\right|_{\Gamma}\right\|_{Z}+\|\gamma\|_{L^{\infty}(\Gamma)}\left\|\left.\tilde{u}_{1, h}^{k}\right|_{\Gamma}\right\| Z\right)\left\|\left.\tilde{u}_{1, h}^{k}\right|_{\Gamma}\right\|_{Z}
$$

wegen der Koerzivität von $a_{1, h}^{u p}$ sowie der Stetigkeit von $S_{2, h}^{(\gamma)}($ siehe (7.14) bzw. (7.19)) mit $m_{2, h}=\left(1+M_{2}+h C_{\infty, 2}\right) C_{E_{2, h}}^{2}$.

Zieht man die Äquivalenz (7.22) heran, so folgt aus der vorausgegangenen Ungleichung

$$
\begin{aligned}
\alpha_{u p, 1}\left\|\tilde{u}_{1, h}^{k}\right\|_{L^{2}\left(0, T ; V_{1}\right)}^{2} \leqslant \frac{1}{\omega_{2}^{*}+\gamma_{0}}\left(\left(m_{2, h}+\|\gamma\|_{L^{\infty}(\Gamma)}\right)\left\|\left.\tilde{u}_{2, h}^{k}\right|_{\Gamma}\right\|_{h}\right. \\
\left.+\|\gamma\|_{L^{\infty}(\Gamma)}\left\|\left.\tilde{u}_{1, h}^{k}\right|_{\Gamma}\right\|\right)\left\|\left.\tilde{u}_{1, h}^{k}\right|_{\Gamma}\right\| \|_{h} .
\end{aligned}
$$

Insgesamt erhält man wegen $\left\|\left.\tilde{u}_{2}^{k}\right|_{\Gamma}\right\|_{h} \stackrel{k \rightarrow \infty}{\longrightarrow} 0$ und $\left\|\left.\tilde{u}_{1}^{k}\right|_{\Gamma}\right\|_{h} \stackrel{k \rightarrow \infty}{\longrightarrow} 0$ die Konvergenz $\left(u_{1, h}^{k}, u_{2, h}^{k}\right) \stackrel{k \rightarrow \infty}{\longrightarrow}\left(u_{1, h}, u_{2, h}\right)$ in $L^{2}\left(0, T ; V_{1, h}\right) \times L^{2}\left(0, T ; V_{2, h}\right)$.

\subsection{Numerische Ergebnisse}

In diesem Abschnitt werden Eigenschaften des vorgestellten Dirichlet-Robin-Algorithmus für Konvektions-Diffusions-Reaktions-Probleme anhand numerischer Resultate untersucht. Insbesondere soll das lineare Konvergenzverhalten illustriert werden, das Gegenstand von Satz 7.3.3 bzw. 6.3 .3 ist.

Allen Beispielen dieses Abschnitts liegen zweidimensionale Probleme der Form

$$
u^{\prime}-\varepsilon \Delta u+\nabla \cdot(u b)+c u=f \quad \text { in }(0,1) \times \Omega
$$

über $\Omega=(0,1) \times(0,1)$ mit homogenen Randbedingungen und Anfangsbedingungen $u(0, \cdot)=$ $u_{0}$ zugrunde. Über die Problemdaten $\varepsilon, b, c, f$, die Diskretisierungsparameter $h, \Delta t$ und die Zerlegung des Gebietes $\Omega$ wird jeweils nach untersuchtem Aspekt variiert.

Als Diskretisierung nutzen wir eine verifizierte Finite-Element-Diskretisierung auf Grundlage der Programmpakete MATLAB/FEMLAB statt einer Finite-Volumen-Diskretisierung und verweisen auf den starken Zusammenhang zwischen beiden Diskretisierungen, wie er in Kapitel 3 und Kapitel 4 herausgearbeitet wurde. In konvektionsdominierten Fällen nutzen wir die SUPG-Stabilisierung (siehe etwa [37], zur SUPG-Stabilisierung bei Finite-VolumenDiskretisierung siehe [60]). Als Diskretisierung in der Zeit dient das $\theta$-Schema mit $\theta=1$ (implizites Euler-Verfahren, vergl. 3.4).

Bei der Implementierung der zusätzlichen Austauschrandterme der Robin-Randbedingung (vergl. Gleichung (5.14)) haben wir vereinfachend nicht das volle Skalarprodukt $\langle\cdot, \cdot\rangle_{W}$ sondern das $L^{2}$-Skalarprodukt auf $\Gamma$ herangezogen. Die induzierten Normen beider Skalarprodukte sind aufgrund der endlichen Dimension von $W_{h}$ äquivalent.

Eine Übersicht über die verwendeten Kombinationen von Konvektionsvektoren und Diffusionskoeffizienten sowie die dazugehörigen Abkürzungen zeigt die folgende Tabelle. 


\begin{tabular}{|c|c|c|c|c|c|}
\hline$b=$ & $\frac{1}{\sqrt{2}}(-1,1)^{T}$ & $(0,1)^{T}$ & $(1,0)^{T}$ & $(-1,0)^{T}$ & $(-y, x)^{T}$ \\
\hline$\varepsilon=1$ & $\overline{A_{1}}$ & $\overline{B_{1}}$ & $\overline{C C_{1}}$ & $\overline{D_{1}}$ & $E_{1}$ \\
\hline$\varepsilon=10^{-4}$ & $A_{10^{-4}}$ & $B_{10^{-4}}$ & $C_{10^{-4}}$ & $D_{10^{-4}}$ & $E_{10^{-4}}$ \\
\hline$\varepsilon=10^{-6}$ & $A_{10^{-6}}$ & $B_{10^{-6}}$ & $C_{10^{-6}}$ & $D_{10^{-6}}$ & $E_{10^{-6}}$ \\
\hline
\end{tabular}

Tabelle 7.1: Verwendete Kombinationen von Konvektionsvektoren und Diffusionskoeffizienten

Es werden beide Konvektionsrichtungen $(1,0)^{T}$ und $(-1,0)^{T}$ betrachtet, da der DirichletRobin-Algorithmus prinzipiell unsymmetrisch bezüglich der Zerlegung von $\Omega$ ist.

Beispiel 7.4.1 (Quantitatives Konvergenzverhalten) Wir betrachten die Fälle der obenstehenden Tabelle, jeweils mit dem konstanten Reaktionskoeffizient $c=\frac{1}{2}$ und dem Quellterm

$$
f:(t, x, y) \mapsto e^{-\frac{1}{2} t}\left((y-x) x y-y^{2}+y\right) .
$$

Das Gebiet $\Omega$ zerlegen wir durch $\Omega_{1}:=(0,0.5) \times(0,1), \Omega_{2}:=(0.5,1) \times(0,1)$. Über $\Omega_{1}$ werden die Dirichlet-Probleme, über $\Omega_{2}$ die Robin-Probleme gelöst.

Der Gitterparameter $h$ beträgt in den Berechnungen sämtlicher Fälle für beide Teilgebiete 0.025 und die Zeitschrittweite $\Delta t=0.05$.

Die Abbildung 7.2 zeigt abgetragen über der Anzahl der Iterationen im Dirichlet-RobinAlgorithmus die Entwicklung der Fehler

$$
\begin{aligned}
e_{0}^{k} & :=\left(\sum_{i=1,2}\left\|u_{i, h}^{k}-u_{i, h}\right\|_{L^{2}\left(0, T ; L^{2}\left(\Omega_{i}\right)\right)}^{2}\right)^{\frac{1}{2}}, \\
e_{\infty, \Gamma}^{k} & :=\max _{i=1,2} \frac{\left\|u_{i, h}^{k+1}-u_{i, h}^{k}\right\|_{L^{2}\left(0, T ; L^{\infty}(\Gamma)\right)}}{\left\|u_{i, h}^{k}\right\|_{L^{2}\left(0, T ; L^{\infty}(\Gamma)\right)}} \\
e_{\varepsilon}^{k} & :=\left(\sum_{i=1,2}\left\|u_{i, h}^{k}-u_{i, h}\right\|_{0, T, \varepsilon, \Omega_{i}}^{2}\right)^{\frac{1}{2}} \operatorname{mit}\|v\|_{0, T, \varepsilon, \Omega_{i}}^{2}:=\int_{0}^{T}\|v(t)\|_{\varepsilon, \Omega_{i}}^{2} d t,
\end{aligned}
$$

$\|\cdot\|_{\varepsilon, \Omega_{i}}$ gemäß (1.11), bezüglich der Zweigebietslösung $\left(u_{1, h}, u_{2, h}\right)$, d. h. bezüglich der Restriktionen der globalen diskrete Lösung $u_{h}$ auf $\Omega_{1}, \Omega_{2}$.

Die dargestellten Fehlerentwicklungen zeigen lineare Konvergenz bis unter den Wert $10^{-14}$. Die Iterierung bis zur Maschinengenauigkeit erfolgt hierbei aus analytischen Gründen. In der Praxis ist es ausreichend, die Rechnungen bis zur Größe des Diskretisierungsfehlers zu führen, also deutlich weniger Iterationen auszuführen. Die jeweils verwendeten Relaxationsparameter $\sigma$ und konstanten Austauschrandparameter $\gamma \in \mathbb{R}$ sind unter den Diagrammen angegeben. In den Fällen $\left(A_{1}\right)-\left(E_{1}\right)$ konnte mit $\gamma=0$, also ohne zusätzlichen Term auf dem Austauschrand gerechnet werden, während in den konvektionsdominierten Fällen, entsprechend den theoretischen Ergebnissen aus Abschnitt 6.3 bzw. 7.3, die Wahl eines positiven Austauschrandparameters Voraussetzung für die Konvergenz des Verfahrens war. 
Wie bereits in Abschnitt 6.3 erwähnt, erfordert der sinnvolle Einsatz des Gebietszerlegungsverfahrens in Anwendungen die Wahl eines ausreichend „guten“ $\gamma$. Überlegungen zur optimierten Wahl des Austauschrandparameter wurden hier noch nicht berücksichtigt, dies wird Gegenstand von Kapitel 8 sein.

Alle Ergebnisse dieses Beispiels (bei Größengleichheit von $\Omega_{1}$ und $\Omega_{2}$ ) konnten wir mit der Wahl $\sigma=0.6$ oder $\sigma=0.7$ erreichen. Die Sensibilität des Dirichlet-Robin-Algorithmus gegenüber Änderungen von $\sigma$ stellt sich nach unserer Erfahrung geringer als die gegenüber Änderungen von $\gamma$ dar. Für Untersuchungen zur Wahl von $\sigma$ verweisen wir ebenfalls auf Kapitel 8.

In den Fällen mit konstantem Konvektionsvektor, also $\left(A_{\varepsilon}\right)-\left(D_{\varepsilon}\right)$, ergibt sich für $\varepsilon=10^{-4}$ und $\varepsilon=10^{-6}$ ein im wesentlichen identisches Konvergenzverhalten unter Beibehaltung der Paramter $\sigma$ und $\gamma$. Dies ist ebenso in der Anwendung des Dirichlet-Robin-Algorithmus auf elliptische Probleme zu beobachten (siehe [2]). Im Fall von nicht konstantem Konvektionsvektor, $\left(E_{\varepsilon}\right)$, ergibt sich jedoch eine deutliche Verlangsamung der Konvergenz mit kleiner werdendem $\varepsilon$.

Insgesamt zeigt sich, daß in nicht konvektionsdominierten Fällen sowie in konvektionsdominierten Fällen mit Konvektion durch den Austauschrand selbst mit konstantem $\gamma$ gute Ergebnisse erzielt werden. Allerdings legen die Ergebnisse für $\left(B_{10^{-4}}\right)$ und $\left(B_{10^{-6}}\right)$ sowie die Ergebnisse für $\left(E_{10^{-4}}\right)$ und $\left(E_{10^{-6}}\right)$ die Suche nach einer Strategie zur Wahl optimierter Austauschrandbedingungen nahe.

Abschließend greifen wir noch einige der Rechnungen dieses Beispiels heraus, um die zeitliche Entwicklung der Differenz zwischen den iterativen Teilgebietslösungen und der globalen Lösung für die ersten Iterationsschritte zu demonstrieren. Jeweils für die Fälle $\left(A_{1}\right),\left(A_{10^{-4}}\right)$ - $\left(C_{10^{-4}}\right)$ zeigen die Abbildungen $7.3-7.6$ die Differenz $u_{i, h}^{k}(t)-u_{i, h}(t), i=1$, 2 , für die ersten Iterationsschritte des Dirichlet-Robin-Algorithmus zu den Zeitpunkten $t=0.1,0.5$, 1.0. Gegenüber den biherigen Rechnungen in diesem Beispiel wurde nur der Gitterparameter aus Darstellungsgründen geändert zu $h=0.04$. Im ersten Fall $\left(A_{1}\right)$ tritt der Fehler in $\Omega_{1}$ und $\Omega_{2}$ gleichermaßen auf. Im den konvektionsdominierten Fällen ist der Fehler auf dem Gebiet mit Dirichlet-Randbedingungen deutlich größer. 


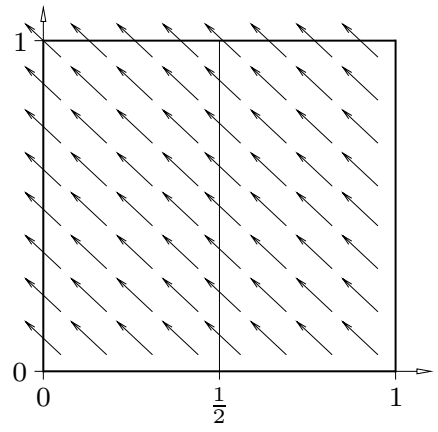

Konvektionsfeld $A$ (skaliert)

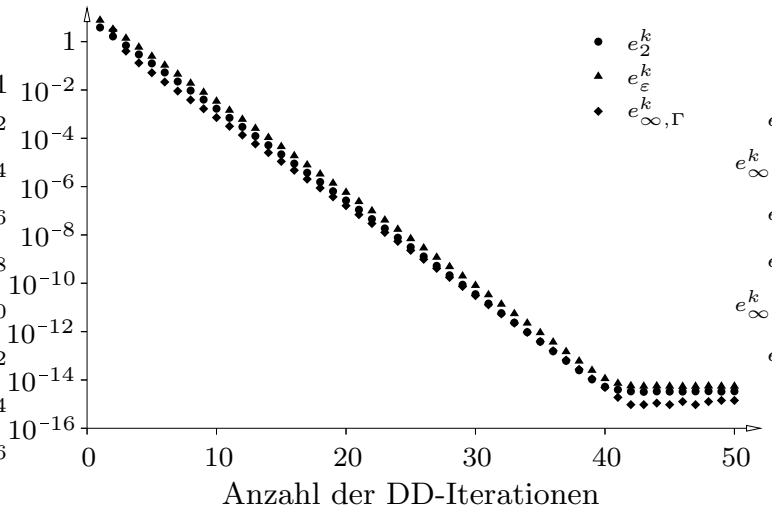

$\left(A_{10^{-4}}\right) \quad \sigma=0.6, \gamma=0.4$

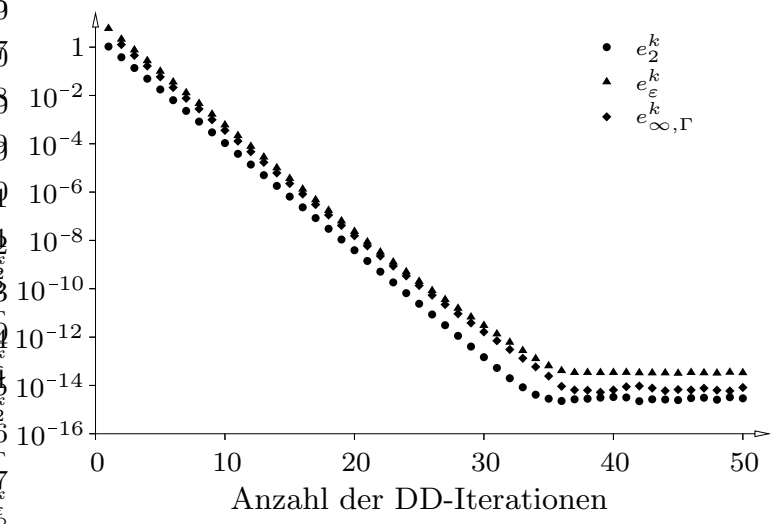

$\left(A_{1}\right) \quad \sigma=0.7, \gamma=0.0$

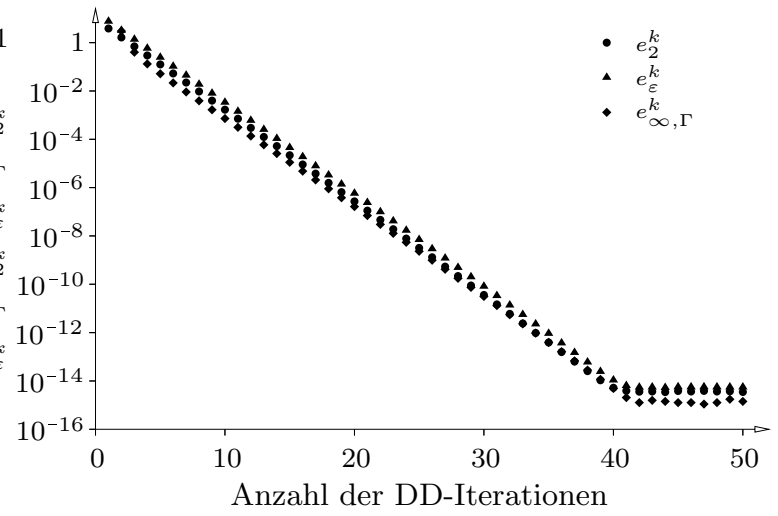

$\left(A_{10^{-6}}\right) \quad \sigma=0.6, \gamma=0.4$

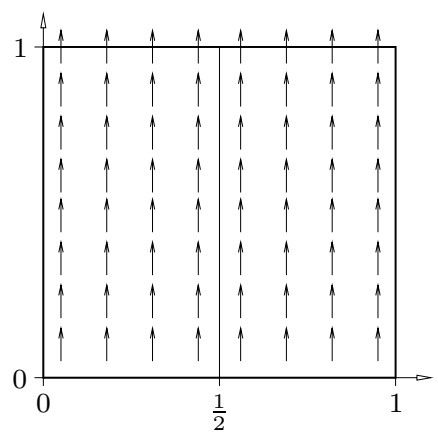

Konvektionsfeld $B$ (skaliert)

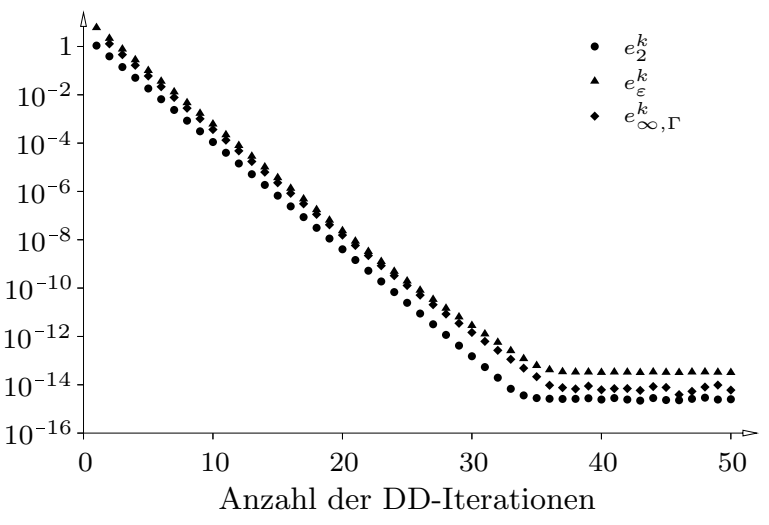

$\left(B_{1}\right) \quad \sigma=0.7, \gamma=0.0$ 


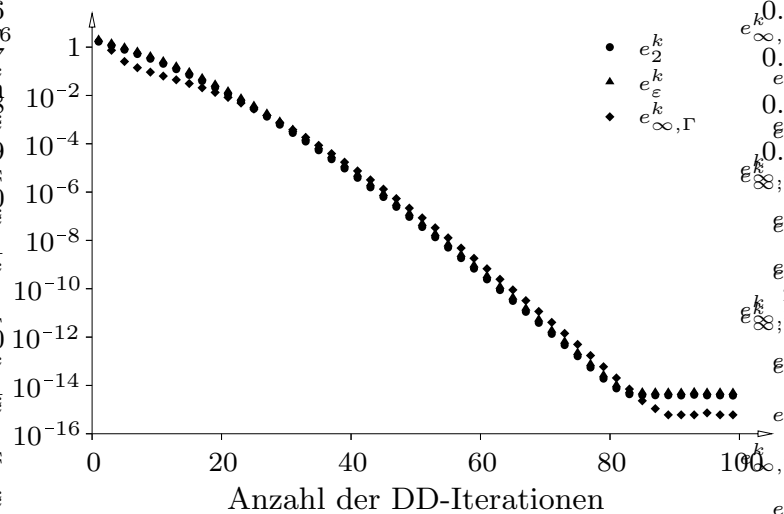

$\left(B_{10^{-4}}\right) \quad \sigma=0.6, \gamma=0.2$

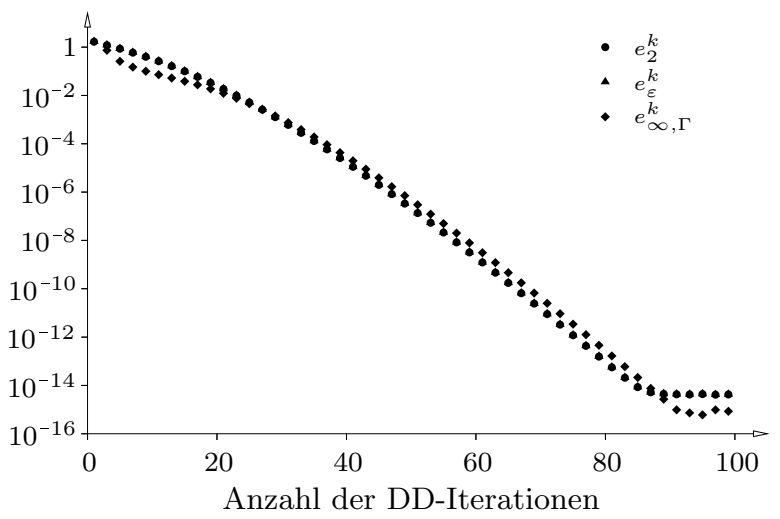

$\left(B_{10^{-6}}\right) \quad \sigma=0.6, \gamma=0.2$

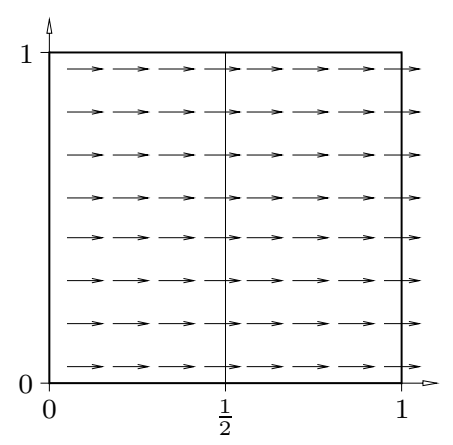

Konvektionsfeld $C$ (skaliert)

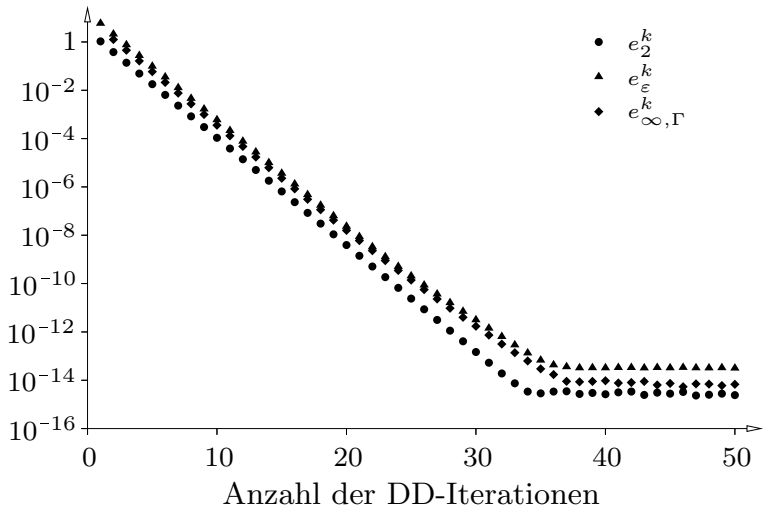

$\left(C_{1}\right) \quad \sigma=0.7, \gamma=0.0$

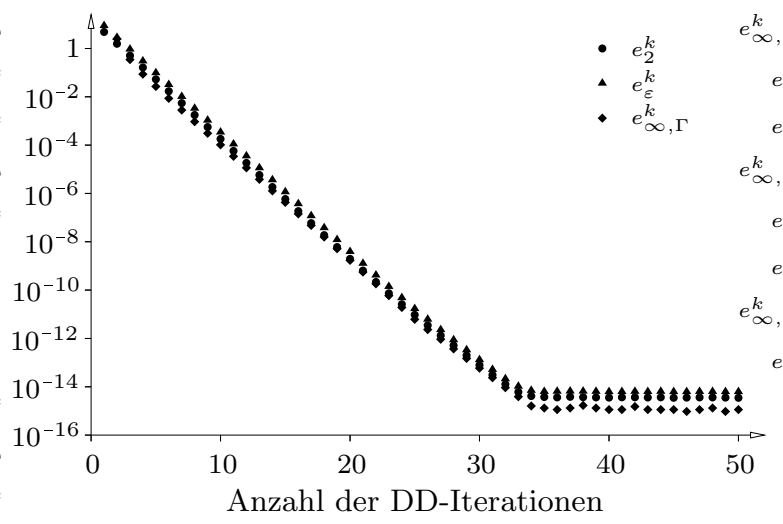

$\left(C_{10^{-4}}\right) \quad \sigma=0.6, \gamma=0.4$

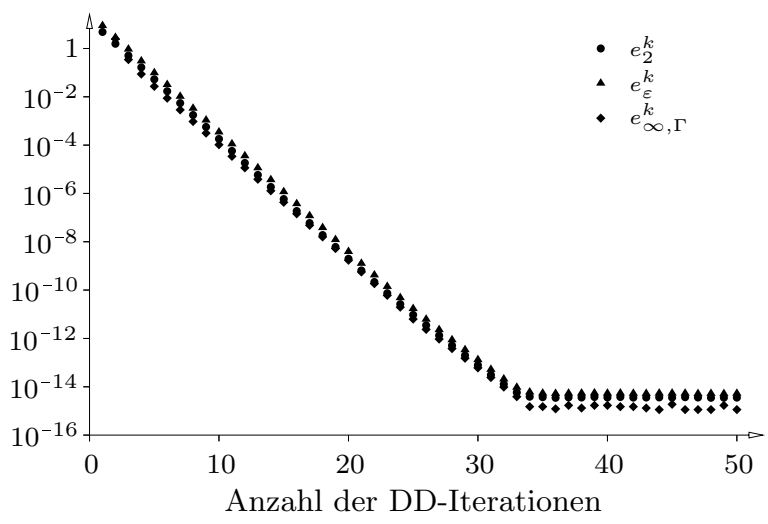

$\left(C_{10^{-6}}\right) \quad \sigma=0.6, \gamma=0.4$ 


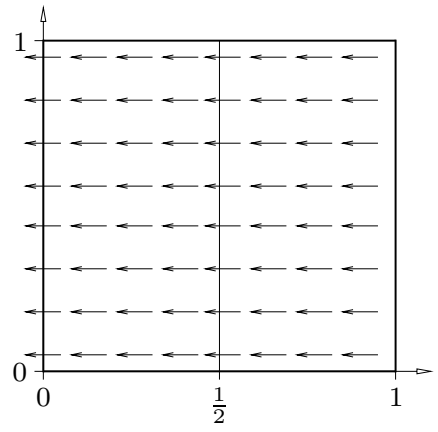

Konvektionsfeld $D$ (skaliert)

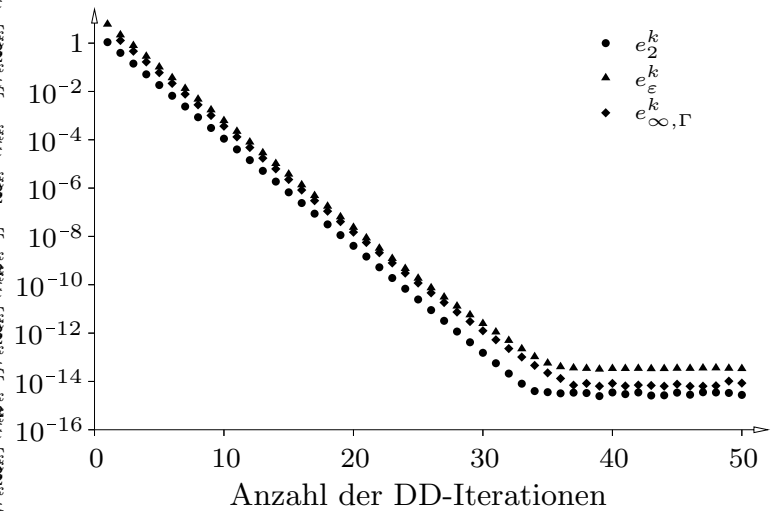

$\left(D_{1}\right) \quad \sigma=0.7, \gamma=0.0$

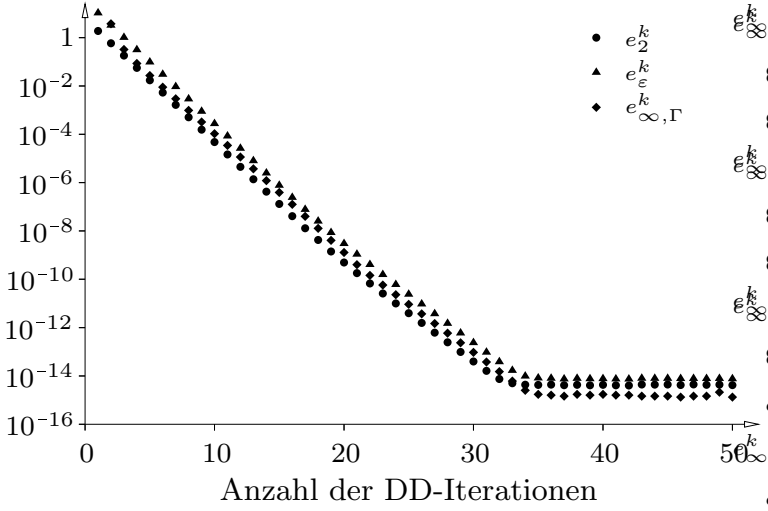

$\left(D_{10^{-4}}\right) \quad \sigma=0.6, \gamma=0.4$

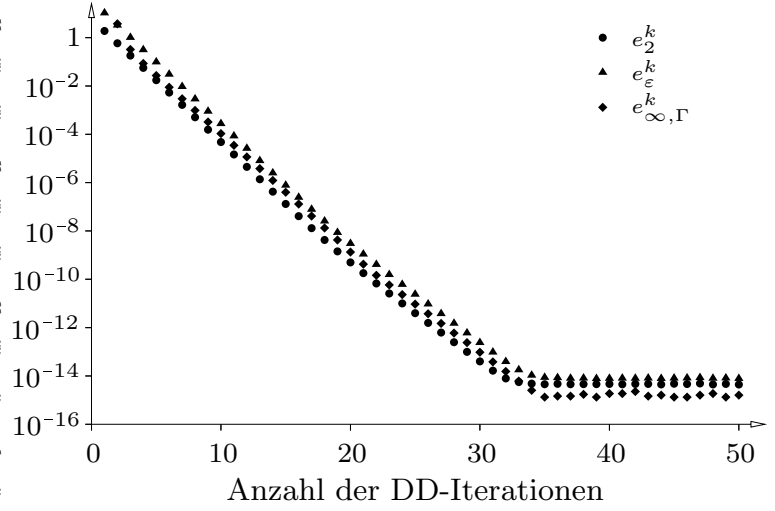

$\left(D_{10^{-6}}\right) \quad \sigma=0.6, \gamma=0.4$

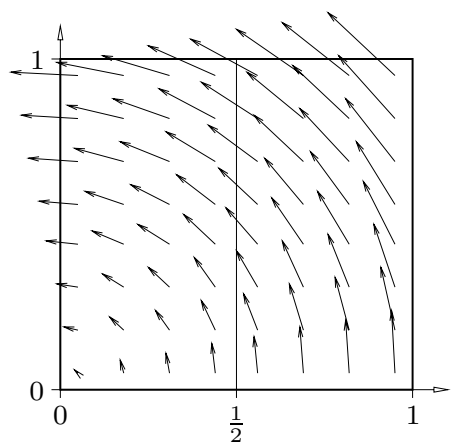

Konvektionsfeld $E$ (skaliert)

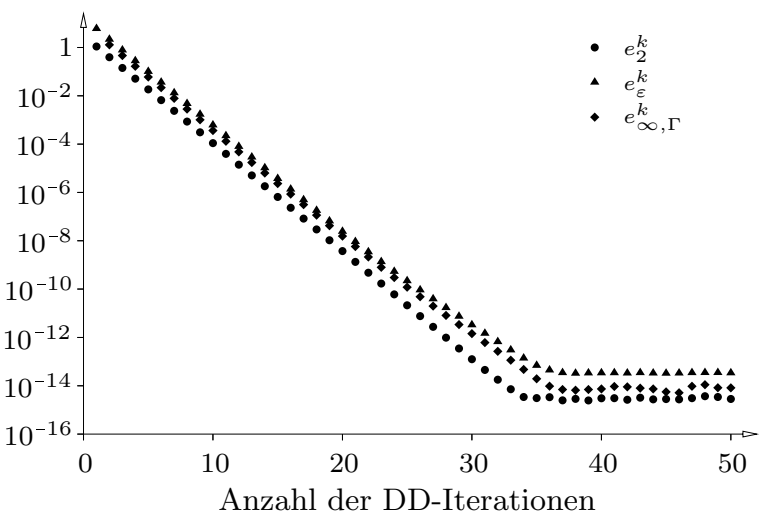

$\left(E_{1}\right) \quad \sigma=0.7, \gamma=0.0$ 


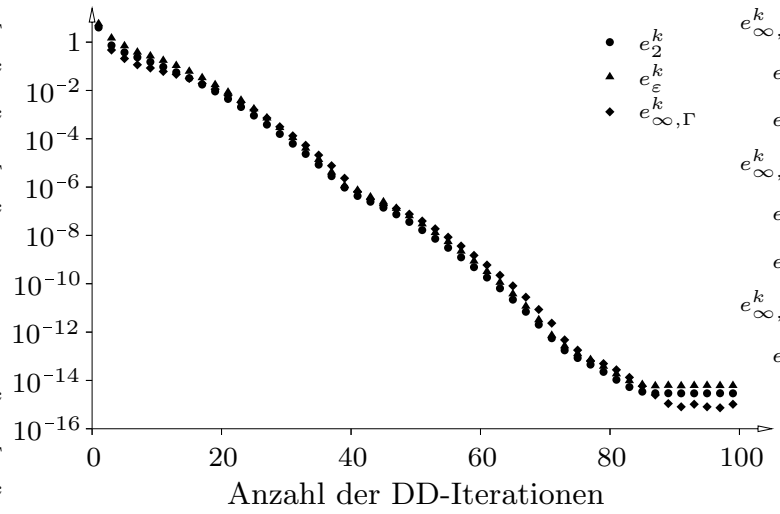

$\left(E_{10^{-4}}\right) \quad \sigma=0.6, \gamma=0.2$

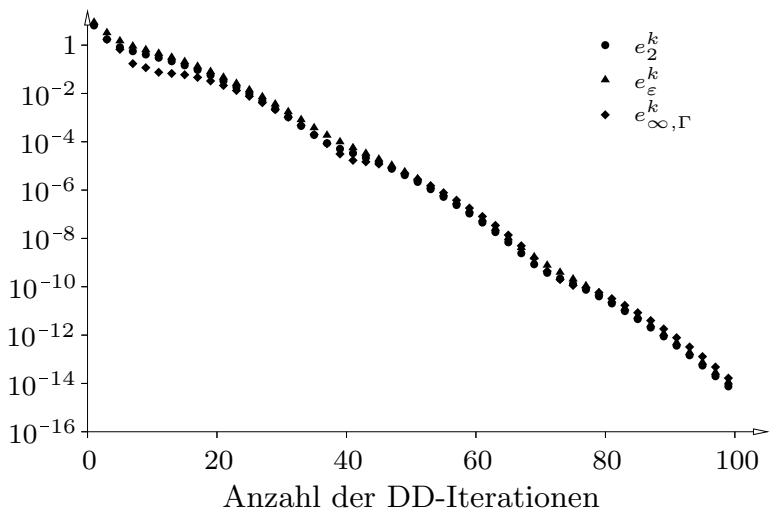

$\left(E_{10^{-6}}\right) \quad \sigma=0.6, \gamma=0.2$

Abbildung 7.2: Die Fehler $e_{2}^{k}, e_{\varepsilon}^{k}$ und $e_{\infty, \Gamma}^{k}$ in Abhängigkeit von der Anzahl der DD-Iterationen für die Fälle $\left(A_{\varepsilon}\right)-\left(E_{\varepsilon}\right), \varepsilon=1,10^{-4}, 10^{-6}$, laut Tabelle 7.1 . 


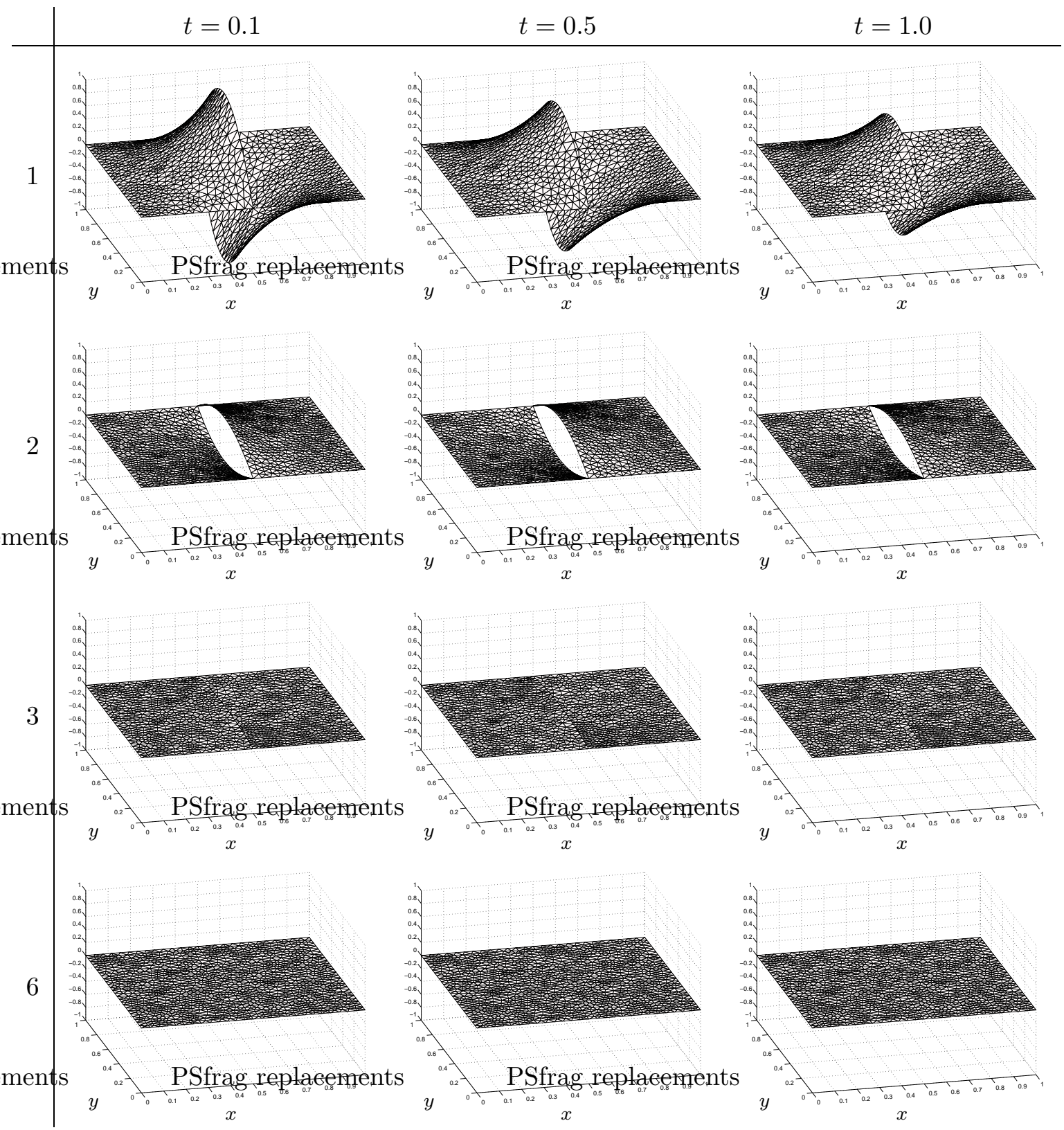

Abbildung 7.3: Differenz $u_{i, h}^{k}(t)-u_{i, h}(t), i=1,2$, im Fall $\left(A_{1}\right)$ zu unterschiedlichen Zeitpunkten für die Iterationsschritte 1,2,3,4 und 6 . 


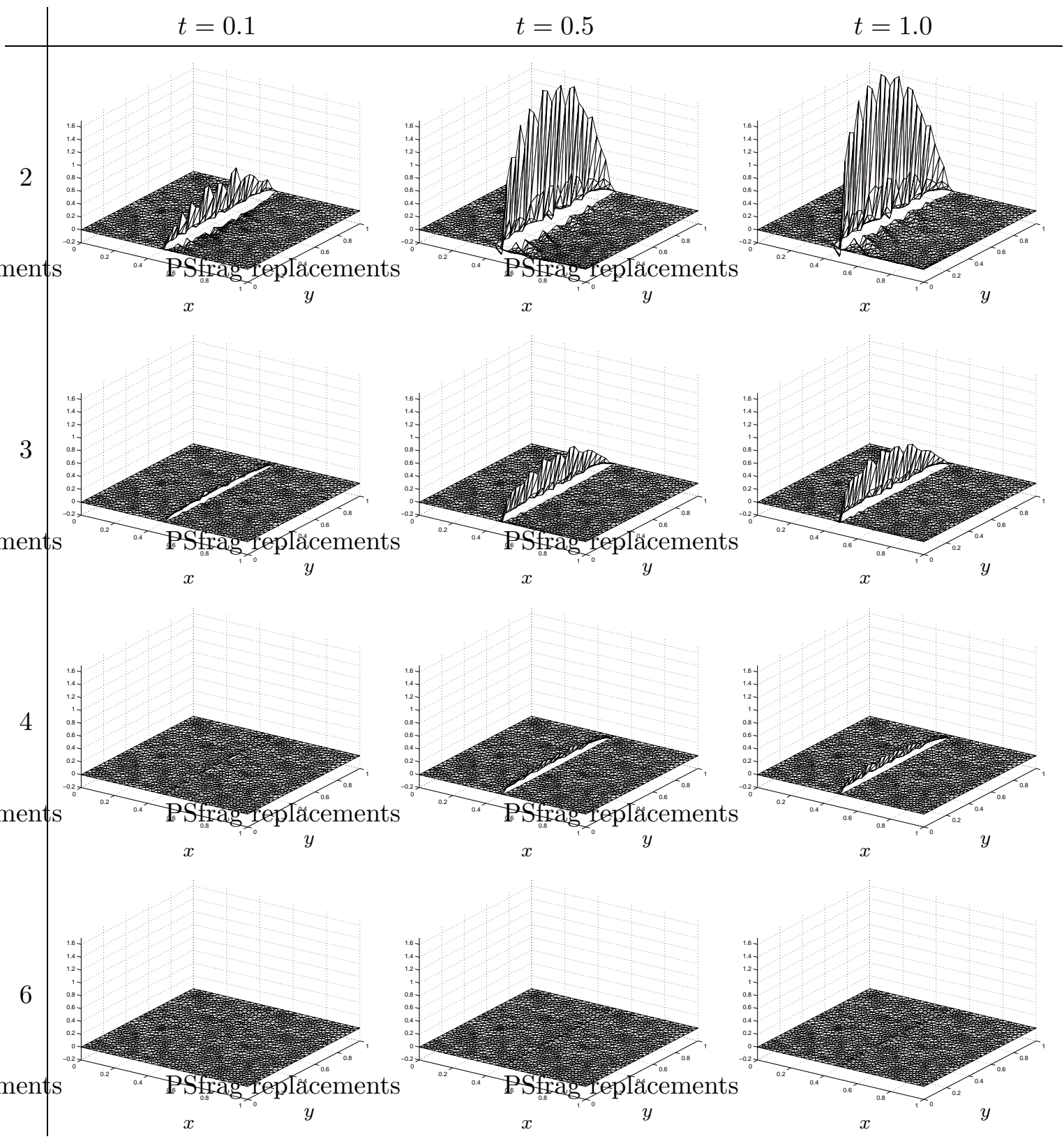

Abbildung 7.4: Differenz $u_{i, h}^{k}(t)-u_{i, h}(t), i=1,2$, im Fall $\left(A_{10^{-4}}\right)$ zu unterschiedlichen Zeitpunkten für die Iterationsschritte 2,3,4 und 6 . 


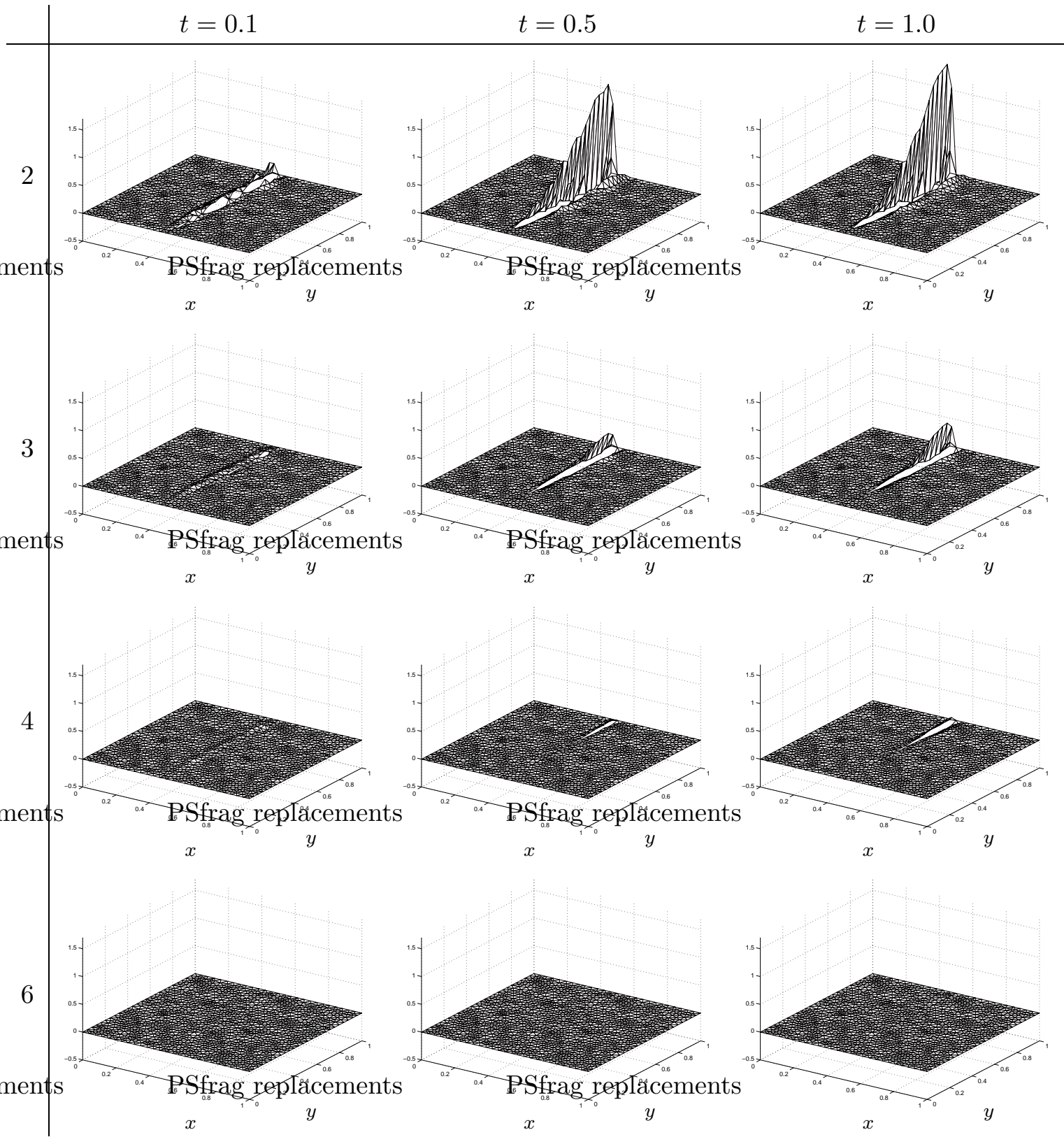

Abbildung 7.5: Differenz $u_{i, h}^{k}(t)-u_{i, h}(t), i=1,2$, im Fall $\left(B_{10^{-4}}\right)$ zu unterschiedlichen Zeitpunkten für die Iterationsschritte 2,3,4 und 6 . 


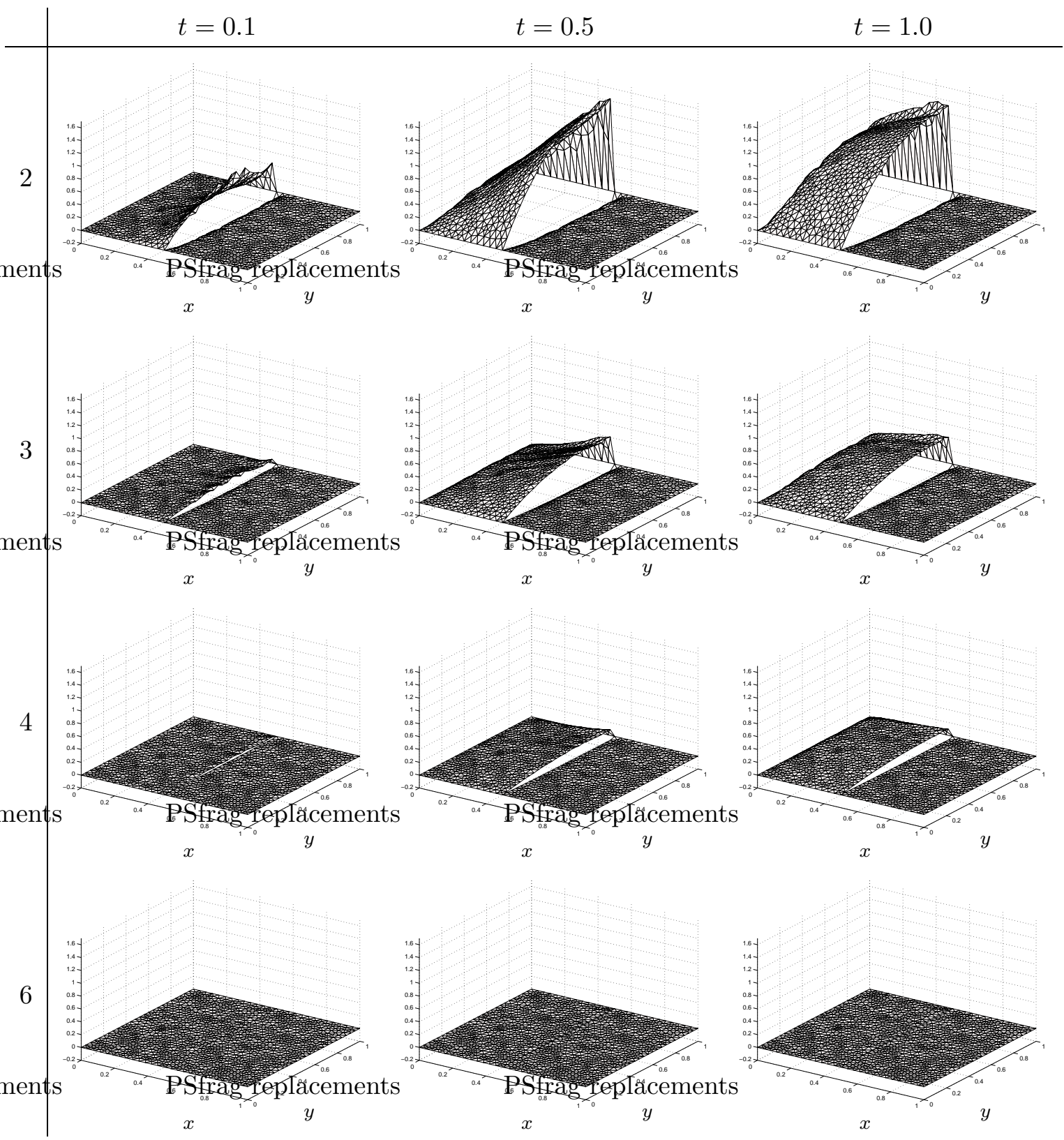

Abbildung 7.6: Differenz $u_{i, h}^{k}(t)-u_{i, h}(t), i=1,2$, im Fall $\left(C_{10^{-4}}\right)$ zu unterschiedlichen Zeitpunkten für die Iterationsschritte $2,3,4$ und 6 .

Beispiel 7.4.2 (Einfluß der Gitterweite auf die Konvergenz) In diesem Beispiel untersuchen wir den Einfluß des Gitterparameters $h$ auf die Konvergenz des Dirichlet-RobinAlgorithmus anhand der Fälle $\left(A_{\varepsilon}\right),\left(B_{\varepsilon}\right)$ und $\left(E_{\varepsilon}\right), \varepsilon=1,10^{-4}$, gemäß Tabelle 7.1 mit dem Reaktionskoeffizient $c=0$. Die Zerlegung von $\Omega$ erfolgt bei $x=0.5$ wie im Beispiel 7.4.1, und die Zeitschrittweite $\Delta t$ beträgt wiederum 0.05 .

Die Tabelle 7.2 gibt jeweils die Anzahl der Iterationen wieder, die nötig sind, um den Fehler $e_{0}^{k}$ unter die Schranken $10^{-4}, 10^{-6}, 10^{-8}$ und $10^{-10} \mathrm{zu}$ bringen. Dabei bedeutet ein Strich ,-_", daß die jeweilige Fehlerschranke innerhalb von 120 Iterationen nicht unterschritten wurde. 
Die verwendeten Werte von $\sigma$ und $\gamma$ wurden aus Beispiel 7.4.1 übernommen.

\begin{tabular}{|c|c|c|c|c|c|c|c|}
\hline \multirow{2}{*}{ Fall } & \multirow{2}{*}{$\sigma$} & \multirow[b]{2}{*}{$\gamma$} & \multirow{2}{*}{$h$} & \multicolumn{4}{|c|}{ Anzahl Iterationen zur Erreichung $e_{0}^{k}<$} \\
\hline & & & & $10^{-4}$ & $10^{-6}$ & $10^{-8}$ & $10^{-10}$ \\
\hline \multirow{5}{*}{$\left(A_{1}\right)$} & \multirow{5}{*}{0.7} & \multirow{5}{*}{0.0} & 0.16 & 11 & 15 & 20 & 24 \\
\hline & & & 0.08 & 11 & 15 & 20 & 25 \\
\hline & & & 0.04 & 11 & 15 & 20 & 24 \\
\hline & & & 0.02 & 11 & 15 & 20 & 24 \\
\hline & & & 0.01 & 11 & 15 & 20 & 24 \\
\hline \multirow{5}{*}{$\left(A_{10^{-4}}\right)$} & \multirow{5}{*}{0.6} & \multirow{5}{*}{0.4} & 0.16 & 11 & 15 & 20 & 25 \\
\hline & & & 0.08 & 12 & 16 & 20 & 25 \\
\hline & & & 0.04 & 14 & 19 & 24 & 28 \\
\hline & & & 0.02 & 15 & 20 & 25 & 30 \\
\hline & & & 0.01 & 15 & 20 & 26 & 31 \\
\hline \multirow{5}{*}{$\left(B_{1}\right)$} & \multirow{5}{*}{0.7} & \multirow{5}{*}{0.0} & 0.16 & 11 & 15 & 20 & 24 \\
\hline & & & 0.08 & 11 & 15 & 20 & 25 \\
\hline & & & 0.04 & 11 & 15 & 20 & 24 \\
\hline & & & 0.02 & 11 & 15 & 20 & 24 \\
\hline & & & 0.01 & 11 & 15 & 20 & 24 \\
\hline \multirow{5}{*}{$\left(B_{10^{-4}}\right)$} & \multirow{5}{*}{0.6} & \multirow{5}{*}{0.2} & 0.16 & 12 & 15 & 20 & 25 \\
\hline & & & 0.08 & 17 & 21 & 25 & 30 \\
\hline & & & 0.04 & 25 & 33 & 39 & 45 \\
\hline & & & 0.02 & 39 & 50 & 60 & 69 \\
\hline & & & 0.01 & 54 & - & - & - \\
\hline \multirow{5}{*}{$\left(E_{1}\right)$} & \multirow{5}{*}{0.7} & \multirow{5}{*}{0.0} & 0.16 & 11 & 15 & 20 & 24 \\
\hline & & & 0.08 & 11 & 15 & 20 & 25 \\
\hline & & & 0.04 & 11 & 15 & 20 & 24 \\
\hline & & & 0.02 & 11 & 15 & 20 & 24 \\
\hline & & & 0.01 & 11 & 15 & 20 & 24 \\
\hline \multirow{5}{*}{$\left(E_{10^{-4}}\right)$} & \multirow{5}{*}{0.6} & \multirow{5}{*}{0.2} & 0.16 & 14 & 18 & 23 & 28 \\
\hline & & & 0.08 & 18 & 22 & 27 & 32 \\
\hline & & & 0.04 & 23 & 32 & 40 & 47 \\
\hline & & & 0.02 & 29 & 39 & 49 & 59 \\
\hline & & & 0.01 & 32 & 43 & 53 & 64 \\
\hline
\end{tabular}

Tabelle 7.2: Äbhängigkeit der Konvergenzgeschwindigkeit von der Gitterweite für die Fälle $\left(A_{\varepsilon}\right),\left(B_{\varepsilon}\right)$ und $\left(E_{\varepsilon}\right), \varepsilon=1,10^{-4}$

In den Fällen $\left(A_{1}\right),\left(B_{1}\right)$ sowie $\left(E_{1}\right)$ ergibt sich die Unabhängigkeit der Konvergenz vom Gitterparameters $h$, und der Konvergenzverlauf ist in allen drei Fällen identisch (letzteres war bereits im Beispiel 7.4.1 zu sehen). 
Dagegen ändert sich in den konvektionsdominanten Fällen die Konvergenzrate mit $h$ deutlich, besonders bei $\left(B_{10^{-4}}\right)$ und $\left(E_{10^{-4}}\right)$. Mit kleiner werdendem $h$ wächst die entsprechende Iterationszahl, teilweise auf mehr als das Doppelte innerhalb des Intervalls von $h$. In Kapitel 8 werden wir jedoch bei optimal gewähltem Austauschrandparameters $\gamma$ auch in konvektionsdominanten Fällen Unabhängigkeit von $h$ erhalten (siehe Beispiel 8.3.2).

Beispiel 7.4.3 (Abhängigkeit der Konvergenz vom Teilgebietsverhältnis) Es soll nun anhand derselben Fälle wie im Beispiel 7.4.2 der Einfluß des Größenverhältnisses der beiden Teilgebiete dargestellt werden. Dazu werden die Zerlegungen

$$
\Omega_{1}:=(0, l) \times(0,1), \quad \Omega_{2}:=(l, 1) \times(0,1), \quad l=0.1,0.2,0.5,0.8,0.9
$$

des Gebiets $\Omega$ herangezogen und über $\Omega_{1}$ die Dirichlet-Probleme, über $\Omega_{2}$ die Robin-Probleme gelöst, siehe Abbildung 7.7. Diskretisiert wird mit dem Gitterparameter $h=0.025$ und der Zeitschrittweite $\Delta t=0.05$.

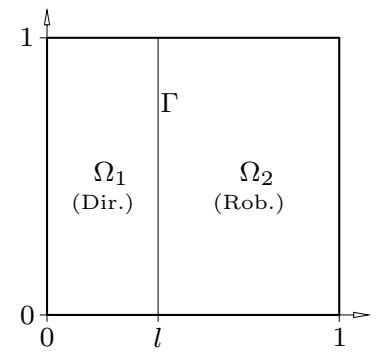

Abbildung 7.7: Zerlegung des Einheitsquadrates in $\Omega_{1}=(0, l) \times(0,1)$ und $\Omega_{2}=(l, 1) \times(0,1)$

Die Tabelle 7.3 listet für die angegebenen Werte von $l$ jeweils die Anzahl der Iterationen auf, die nötig sind, um den Fehler $e_{0}^{k}$ unter die Schranken $10^{-4}, 10^{-6}, 10^{-8}$ und $10^{-10}$ zu bringen. Wiederum bedeutet „-“, daß die jeweilige Fehlerschranke innerhalb von 120 Iterationen nicht unterschritten wurde.

In den Fällen $\left(A_{1}\right),\left(B_{1}\right)$ sowie $\left(E_{1}\right)$ ändert sich mit Variation der Werte von $l$ die Konvergenz erheblich. Diese ist hier um so schneller, je größer das Dirichlet-Teilgebiet, $\Omega_{1}$, ist. Für $l=0.1$ zeigt sich jeweils keine Konvergenz. Auch im Fall $\left(E_{10^{-4}}\right)$ besteht, wenngleich schwächer ausgeprägt, dieser Einfluß. In den Fällen $\left(A_{10^{-4}}\right)$ und $\left(B_{10^{-4}}\right)$ ist die Konvergenzgeschwindigkeit hingegen im wesentlichen gleich für alle Werte von $l$.

Im Fall konstanter Koeffizienten $b$ und $c$ bleibt also die Konvergenz unbeinflußt, sofern $\frac{|b|}{\varepsilon}$ groß gegenüber $l$ ist. Diesen Zusammenhang zwischen der Wahl von $l$ und der Konvergenz des Verfahrens werden die Untersuchungen zur Konvergenzratenfunktion in den Abschnitten 8.1 und 8.2 erhellen. 


\begin{tabular}{|c|c|c|c|c|c|c|c|}
\hline \multirow{2}{*}{ Fall } & \multirow{2}{*}{$\sigma$} & \multirow{2}{*}{$\gamma$} & \multirow{2}{*}{$l$} & \multicolumn{4}{|c|}{ Anzahl Iterationen zur Erreichung $e_{0}^{k}<$} \\
\hline & & & & $10^{-4}$ & $10^{-6}$ & $10^{-8}$ & $10^{-10}$ \\
\hline \multirow{5}{*}{$\left(A_{1}\right)$} & \multirow{5}{*}{0.7} & \multirow{5}{*}{0.0} & 0.1 & - & - & - & - \\
\hline & & & 0.2 & 37 & 49 & 60 & 70 \\
\hline & & & 0.5 & 11 & 15 & 20 & 24 \\
\hline & & & 0.8 & 7 & 11 & 15 & 19 \\
\hline & & & 0.9 & 5 & 9 & 13 & 17 \\
\hline \multirow{5}{*}{$\left(A_{10^{-4}}\right)$} & \multirow{5}{*}{0.6} & \multirow{5}{*}{0.4} & 0.1 & 12 & 17 & 22 & 27 \\
\hline & & & 0.2 & 12 & 17 & 22 & 27 \\
\hline & & & 0.5 & 12 & 17 & 22 & 27 \\
\hline & & & 0.8 & 10 & 15 & 20 & 25 \\
\hline & & & 0.9 & 9 & 14 & 20 & 25 \\
\hline \multirow{5}{*}{$\left(B_{1}\right)$} & \multirow{5}{*}{0.7} & \multirow{5}{*}{0.0} & 0.1 & - & - & - & - \\
\hline & & & 0.2 & 37 & 49 & 60 & 70 \\
\hline & & & 0.5 & 11 & 15 & 20 & 24 \\
\hline & & & 0.8 & 7 & 11 & 15 & 20 \\
\hline & & & 0.9 & 5 & 9 & 13 & 17 \\
\hline \multirow{5}{*}{$\left(B_{10^{-4}}\right)$} & \multirow{5}{*}{0.6} & \multirow{5}{*}{0.2} & 0.1 & 26 & 36 & 44 & 52 \\
\hline & & & 0.2 & 24 & 33 & 42 & 49 \\
\hline & & & 0.5 & 24 & 34 & 42 & 50 \\
\hline & & & 0.8 & 24 & 34 & 43 & 50 \\
\hline & & & 0.9 & 26 & 36 & 45 & 52 \\
\hline \multirow{5}{*}{$\left(E_{1}\right)$} & \multirow{5}{*}{0.7} & \multirow{5}{*}{0.0} & 0.1 & - & - & - & - \\
\hline & & & 0.2 & 37 & 49 & 60 & 70 \\
\hline & & & 0.5 & 11 & 15 & 20 & 24 \\
\hline & & & 0.8 & 7 & 11 & 15 & 20 \\
\hline & & & 0.9 & 5 & 9 & 13 & 17 \\
\hline \multirow{5}{*}{$\left(E_{10^{-4}}\right)$} & \multirow{5}{*}{0.6} & \multirow{5}{*}{0.2} & 0.1 & 30 & 46 & 58 & 69 \\
\hline & & & 0.2 & 24 & 36 & 45 & 55 \\
\hline & & & 0.5 & 21 & 33 & 44 & 54 \\
\hline & & & 0.8 & 17 & 25 & 33 & 40 \\
\hline & & & 0.9 & 15 & 21 & 28 & 33 \\
\hline
\end{tabular}

Tabelle 7.3: Äbhängigkeit der Konvergenzgeschwindigkeit vom Teilgebietsverhältnis für die Fälle $\left(A_{\varepsilon}\right),\left(B_{\varepsilon}\right)$ und $\left(E_{\varepsilon}\right), \varepsilon=1,10^{-4}$ 


\section{Kapitel 8}

\section{Optimale Austauschrandbedingungen}

Die numerischen Resultate des vorausgegangenen Abschnitts stehen im Einklang mit der Analyse des Dirichlet-Robin-Algorithmus aus Kapitel 6 bzw. Abschnitt 7.3. Jedoch treten besonders in konvektionsdominanten Fällen mit Konvektionsrichtung parallel zum Austauschrand oder nicht konstanter Konvektion unbefriedigende Konvergenzraten zu Tage. Daher untersuchen wir in diesem Kapitel eine Strategie zur bezüglich der Konvergenzrate optimierten Wahl von $\gamma$.

Wir stellen eine solche Strategie erst für eindimensionale Probleme dar und übertragen unser Vorgehen dann auf zweidimensionale Probleme. Es handelt sich dabei um die Approximation von Randoperatoren, Pseudo-Differentialoperatoren, wie sie auch als absorbierende Randbedingungen genutzt werden, durch lokale Operatoren, deren Fourier-Transformierte Polynome sind.

Abschließend vergleichen wir auf diese Weise bestimmte Austauschrandparameter $\gamma$ mit Ergebnissen aus numerischen Tests mit dem Dirichlet-Robin-Algorithmus und betrachten die Auswirkungen der optimierten Wahl von $\gamma$ auf das Gebietszerlegungsverfahren.

\subsection{Untersuchung des Dirichlet-Robin-Algorithmus im eindi- mensionalen Fall}

Den Inhalt dieses Abschnitts bildet eine Analyse unseres Gebietszerlegungsverfahrens für eindimensionale Konvektions-Diffusions-Reaktions-Gleichungen. Wir orientieren uns an der Arbeit [26], insbesondere den Untersuchungen dort zur Lösung zeitabhängiger, eindimensionaler Probleme mit Hilfe der Schwarz-Waveform-Relaxation für nicht überlappende Teilgebiete. Nach Fourier-Transformation der Problemgleichungen bezüglich der Zeit kann man, sofern man sich auf Probleme mit konstanten Koeffizienten beschränkt, für jeden Iterationsschritt eine exakte Lösung angeben. Daraus läßt sich ein optimaler Randoperator ableiten, so daß das Gebietszerlegungsverfahren (bei zwei Teilgebieten) in zwei Schritten konvergiert. 
Wir übertragen diese Vorgehen auf unseren Dirichlet-Robin-Algorithmus. Betrachtet werden also Konvektions-Diffusions-Reaktions-Probleme mit konstanten Koeffizienten über dem Einheitsintervall $\Omega:=(0,1)$, nämlich

$$
\left\{\begin{array}{l}
\partial_{t} u+L u=f \quad \text { in }(0, T) \times \Omega \\
u(t, 0)=u(t, 1)=0 \\
u(0, x)=u_{0}(x)
\end{array}\right.
$$

wobei hier

$$
L u=-\varepsilon u_{x x}+b u_{x}+c u
$$

mit $\varepsilon>0, b, c \geqslant 0$. Die Zerlegung in zwei Teilintervalle geschieht durch $\Omega_{1}:=(0, l)$ und $\Omega_{2}:=(l, 1)$ für ein $l \in \Omega$. Den künstlichen Rand $\Gamma$ bildet also die Menge $\{l\}$, siehe Abbildung 8.1 .

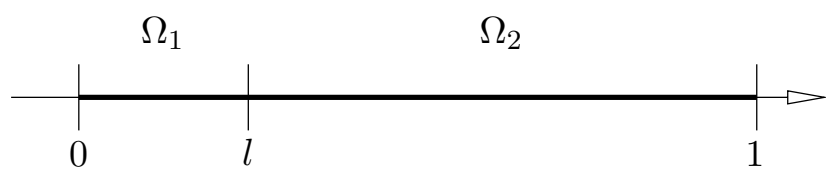

Abbildung 8.1: Die Zerlegung des Einheitsintervalls in $\Omega_{1}=(0, l)$ und $\Omega_{2}=(l, 1)$

Der Dirichlet-Robin-Algorithmus (5.13) - (5.15) gewinnt dann die Form (siehe Bemerkung 5.2 .2

$$
\begin{aligned}
& \left\{\begin{array}{l}
\partial_{t} u_{1}^{k}+L u_{1}^{k}=f \quad \text { in }(0, T) \times \Omega_{1} \\
u_{1}^{k}(t, 0)=0 \\
u_{1}^{k}(t, l)=g^{k-1}(t)
\end{array}\right. \\
& \left\{\begin{array}{l}
\partial_{t} u_{2}^{k}+L u_{2}^{k}=f \quad \text { in }(0, T) \times \Omega_{2} \\
u_{2}^{k}(t, 1)=0 \\
\varepsilon\left(u_{2}^{k}\right)_{x}(t, l)-\left(\frac{1}{2} b-\gamma\right) u_{2}^{k}(t, l)=\varepsilon\left(u_{1}^{k}\right)_{x}(t, l)-\left(\frac{1}{2} b-\gamma\right) u_{1}^{k}(t, l)
\end{array}\right.
\end{aligned}
$$

mit den Anfangsbedingungen $u_{j}^{k}(0, x)=\left.u_{0}\right|_{\Omega_{i}}(x), j=1,2$, sowie

$$
g^{k}(t)=\sigma u_{2}^{k}(t, l)+(1-\sigma) g^{k-1}(t) .
$$

Analog zum Vorgehen in [26] ersetzen wir in unserem iterativen Gebietszerlegungsverfahren den $\gamma$-Term der Robin-Randbedingung durch einen allgemeineren (noch zu bestimmenden) 
linearen Randoperator $\Lambda$ :

$$
\varepsilon\left(u_{2}^{k}\right)_{x}(t, l)-\frac{1}{2} b u_{2}^{k}(t, l)+\Lambda\left(u_{2}^{k}(t, l)\right)=\varepsilon\left(u_{1}^{k}\right)_{x}(t, l)-\frac{1}{2} b u_{1}^{k}(t, l)+\Lambda\left(u_{1}^{k}(t, l)\right)
$$

Nach Lemma 6.1.1 reproduziert sich die Relaxationsgleichung für die Differenzenfolge $\left(\left(\tilde{u}_{1}^{k}, \tilde{u}_{2}^{k}\right)\right)_{k \in \mathbb{N}}$ mit $\tilde{u}_{j}^{k}:=u_{j}^{k}-\left.u\right|_{\Omega_{j}}, j=1,2$. Setzt man $\tilde{g}^{k-1}:=\tilde{u}_{1}^{k}(\cdot, l), k \geqslant 1$, so ist daher

$$
\tilde{g}^{k}(t)=\sigma \tilde{u}_{2}^{k}(t, l)+(1-\sigma) \tilde{g}^{k-1}(t)
$$

Desweiteren ergeben sich über den Teilgebieten homogene Gleichungen mit analogen Randbedingungen am Austauschrand:

$$
\begin{aligned}
& \left\{\begin{array}{l}
\partial_{t} \tilde{u}_{1}^{k}+L \tilde{u}_{1}^{k}=0 \quad \text { in }(0, T) \times \Omega_{1} \\
\tilde{u}_{1}^{k}(t, 0)=0 \\
\tilde{u}_{1}^{k}(t, l)=\tilde{g}^{k-1}(t)
\end{array}\right. \\
& \left\{\begin{array}{l}
\partial_{t} \tilde{u}_{2}^{k}+L \tilde{u}_{2}^{k}=0 \quad \text { in }(0, T) \times \Omega_{2} \\
\tilde{u}_{2}^{k}(t, 1)=0 \\
\varepsilon\left(\tilde{u}_{2}^{k}\right)_{x}(t, l)-\frac{1}{2} b \tilde{u}_{2}^{k}(t, l)+\Lambda\left(\tilde{u}_{2}^{k}(t, l)\right)=\varepsilon\left(\tilde{u}_{1}^{k}\right)_{x}(t, l)-\frac{1}{2} b \tilde{u}_{1}^{k}(t, l)+\Lambda\left(\tilde{u}_{1}^{k}(t, l)\right)
\end{array}\right.
\end{aligned}
$$

mit jeweils verschwindenden Anfangswerten.

Wendet man die Fourier-Transformation $\mathcal{F}$ bezüglich der Zeit $t$ gemäß

$$
\mathcal{F} v(s, x)=\frac{1}{\sqrt{2 \pi}} \int_{\mathbb{R}} e^{-\imath s t} v(t, x) d t .
$$

auf die Gleichungen in (8.5) - (8.7) für $T=\infty$ an, so führt dies auf zwei gewöhnliche Differentialgleichungen (in $x$ ). Mit Hilfe der zugehörigen Lösungen kann man einen optimalen Randoperator $\Lambda$ bestimmen.

Lemma 8.1.1 Der Algorithmus (8.2) - (8.4) mit $T=\infty$ generiert in zwei Iterationen die globale Lösung von (8.1), d. h. $u_{1}^{k}=\left.u\right|_{\Omega_{1}}$ und $u_{2}^{k}=\left.u\right|_{\Omega_{2}}$ für $k \geqslant 2$, falls $\Lambda$ der lineare Operator mit dem zugehörigen Fourier-Symbol

$$
\lambda=\varepsilon \rho((1-\sigma) \operatorname{coth}(\rho(1-l))-\sigma \operatorname{coth}(\rho l))
$$

ist, wobei

$$
\rho=\rho(s)=\frac{1}{2 \varepsilon} \sqrt{b^{2}+4 \varepsilon(c+\imath s)} .
$$


Beweis. Um die Fourier-Transformation anwenden zu können, seien $\tilde{u}_{1}^{k}$, $\tilde{u}_{2}^{k}$ für $t<0$ fortgesetzt durch $\tilde{u}_{1}^{k}(t, x)=\tilde{u}_{2}^{k}(t, x)=0$. Mit dem Differentationssatz für $\mathcal{F}$ folgt wegen $(8.6)_{1}$ bzw. $(8.7)_{1}$

$$
\begin{aligned}
\imath s \mathcal{F} \tilde{u}_{j}^{k}(s, x) & =\mathcal{F}\left(\partial_{t} \tilde{u}_{j}^{k}\right)(s, x) \\
& =\varepsilon \mathcal{F}\left(\tilde{u}_{j}^{k}\right)_{x x}(s, x)-b \mathcal{F}\left(\tilde{u}_{j}^{k}\right)_{x}(s, x)-c \mathcal{F} \tilde{u}_{j}^{k}(s, x) \\
& =\varepsilon\left(\mathcal{F} \tilde{u}_{j}^{k}\right)_{x x}(s, x)-b\left(\mathcal{F} \tilde{u}_{j}^{k}\right)_{x}(s, x)-c \mathcal{F} \tilde{u}_{j}^{k}(s, x)
\end{aligned}
$$

für $x \in \Omega_{j}, j=1,2$.

Man gelangt so zu den zwei gewöhnlichen Differentialgleichungen

$$
\varepsilon\left(w_{j}^{k}\right)_{x x}-b\left(w_{j}^{k}\right)_{x}-(c+\imath s) w_{j}^{k}=0, \quad \mathrm{j}=1,2,
$$

für $w_{j}^{k}(s, x):=\mathcal{F} \tilde{u}_{j}^{k}(s, x)$ in $x$, die allgemein erfüllt werden durch

$$
w_{j}^{k}(s, x)=A_{j}^{k}(s) e^{(\omega+\rho) x}+B_{j}^{k}(s) e^{(\omega-\rho) x}
$$

mit den Koeffizientenfunktionen $A_{j}^{k}, B_{j}^{k}$. Dabei nutzen wir abkürzend

$$
\omega:=\frac{b}{2 \varepsilon}, \quad \rho=\rho(s):=\frac{1}{2 \varepsilon} \sqrt{b^{2}+4 \varepsilon(c+\imath s)} .
$$

Aufgrund der Randbedingung bei $x=0$ und $x=1$ folgt

$$
w_{1}^{k}(s, x)=2 A_{1}^{k}(s) \sinh (\rho x) e^{\omega x}, \quad w_{2}^{k}(s, x)=2 A_{2}^{k}(s) e^{\rho} \sinh (\rho(x-1)) e^{\omega x} .
$$

Zieht man die Randbedingungen am Austauschrand $x=l$ heran, so gelangt man durch Fourier-Tranformation zum einen zu der Gleichung

$$
\mathcal{F} \tilde{g}^{k-1}(s)=w_{1}^{k}(s, l)=2 A_{1}^{k}(s) \sinh (\rho l) e^{\omega l},
$$

die erfüllt wird durch $A_{1}^{k}(s)=\frac{1}{2} \mathcal{F} \tilde{g}^{k-1}(s) e^{-\omega l} \sinh ^{-1}(\rho l)$. Somit erhält $w_{1}^{k}$ die Gestalt

$$
w_{1}^{k}(s, x)=\sinh ^{-1}(\rho l) \mathcal{F} \tilde{g}^{k-1}(s) \sinh (\rho x) e^{\omega(x-l)}
$$

Führt man die Bezeichnung $\Phi(v):=\left.\left(\varepsilon v_{x}-\frac{1}{2} b v+\Lambda(v)\right)\right|_{x=l}$ ein, so ergibt sich zum anderen aus der Randbedingung $(8.7)_{3}$

$$
\mathcal{F} \Phi\left(\tilde{u}_{2}^{k}\right)=\mathcal{F} \Phi\left(\tilde{u}_{1}^{k}\right)
$$

Ist nun $\Lambda$ Operator zum Symbol $\lambda$, also $\mathcal{F}(\Lambda(v))=\lambda \mathcal{F} v$, so folgt

$$
\begin{aligned}
\mathcal{F} \Phi\left(\tilde{u}_{1}^{k}\right)(s) & =\varepsilon\left(w_{1}^{k}\right)_{x}(s, l)-\left(\frac{1}{2} b-\lambda\right) w_{1}^{k}(s, l)=\mathcal{F} \tilde{g}^{k-1}(s)(\varepsilon \rho \operatorname{coth}(\rho l)+\lambda) \\
\mathcal{F} \Phi\left(\tilde{u}_{2}^{k}\right)(s) & =\varepsilon\left(w_{2}^{k}\right)_{x}(s, l)-\left(\frac{1}{2} b-\lambda\right) w_{2}^{k}(s, l) \\
& =2 A_{2}^{k}(s) e^{\rho+\omega l}(\varepsilon \rho \cosh (\rho(l-1))+\lambda \sinh (\rho(l-1)))
\end{aligned}
$$


und somit

$$
A_{2}^{k}(s)=\frac{1}{2} \mathcal{F} \tilde{g}^{k-1}(s) e^{-(\rho+\omega l)} \frac{\varepsilon \rho \operatorname{coth}(\rho l)+\lambda}{\varepsilon \rho \cosh (\rho(l-1))+\lambda \sinh (\rho(l-1))} .
$$

Für die Gestalt von $w_{2}^{k}$ bedeutet dies

$$
w_{2}^{k}(s, x)=\mathcal{F} \tilde{g}^{k-1}(s) \frac{\varepsilon \rho \operatorname{coth}(\rho l)+\lambda}{\varepsilon \rho \cosh (\rho(l-1))+\lambda \sinh (\rho(l-1))} \sinh (\rho(x-1)) e^{\omega(x-l)} .
$$

Es folgt für den Randwert von $w_{2}^{k}(s, x)$ am Austauschrand $x=l$

$$
w_{2}^{k}(s, l)=\mathcal{F} \tilde{g}^{k-1}(s) \frac{\varepsilon \rho \operatorname{coth}(\rho l)+\lambda}{\varepsilon \rho \operatorname{coth}(\rho(l-1))+\lambda} .
$$

Mit der Relaxationsgleichung (8.5) erhält man

$$
\mathcal{F} \tilde{g}^{k}(s)=\left(1-\sigma\left(1+C_{\lambda}(s)\right)\right) \mathcal{F} \tilde{g}^{k-1}(s),
$$

wobei

$$
C_{\lambda}(s):=\frac{\varepsilon \rho \operatorname{coth}(\rho l)+\lambda}{\varepsilon \rho \operatorname{coth}(\rho(1-l))-\lambda} .
$$

Wegen

$$
\begin{aligned}
1-\sigma\left(1+C_{\lambda}(s)\right)=0 & \Longleftrightarrow \frac{1-\sigma}{\sigma}=C_{\lambda}(s) \\
& \Longleftrightarrow \lambda=\varepsilon \rho((1-\sigma) \operatorname{coth}(\rho(1-l))-\sigma \operatorname{coth}(\rho l))
\end{aligned}
$$

für alle $s \in \mathbb{R}$ erfüllen $\tilde{u}_{1}^{k}$ und $\tilde{u}_{2}^{k}$ für $k \geqslant 2$ die Probleme (8.6) $)_{1}$ bzw. (8.7) $)_{1}$ mit homogenen Randbedingungen. Dies zeigt die Behauptung.

Randoperatoren, ebenso konstruiert wie der Operator $\Lambda$ aus Lemma 8.1.1, treten auch in anderem Zusammenhang auf, nämlich als absorbierende Randbedingungen bei Berechnungen zu Problemen über unbeschränkten Gebieten (siehe etwa [19] und [36] für hyperbolische Probleme bzw. Konvektions-Diffusions-Probleme). Andere Bezeichnungen sind künstliche, transparente oder offene Randbedingungen.

Dieser Einsatz zur Beschränkung des Rechengebietes kann als Vorlage für den Einsatz als Randbedingung am Austauschrand bei Gebietszerlegungsverfahren angesehen werden. In beiden Fällen wird der entsprechende Rand „künstlich“ eingeführt, die Problemgleichungen sind also über diesen künstlichen Rand hinweg gültig. Die Randbedingungen sollten daher möglichst durchlässig sein. Die Anwendung bei Gebietszerlegungsverfahren für stationäre Konvektions-Diffusions-Probleme wurde erstmals in [16] gezeigt.

In dem hier untersuchten Fall läßt sich zwar speziell bei gleichgewichteter Gebietsteilung und Relaxation, also für $l=\frac{1}{2}$ und $\sigma=\frac{1}{2}, \Lambda$ explizit bestimmen. Für das Fourier-Symbol $\lambda$ aus Lemma 8.1.1 erhält man dann nämlich 


$$
\lambda=(1-2 \sigma) \varepsilon \rho \operatorname{coth}\left(\frac{1}{2} \rho\right)=0 .
$$

Somit ist hier der Nulloperator optimaler Randoperator. Jedoch spiegelt sich dieses Verhalten in den numerischen Test nicht wieder, siehe Abschnitt 8.3. Wir betrachten daher Werte für den Relaxationsparameter $\sigma$ über das gesamte Intervall $(0,1]$ verteilt.

Im allgemeinen ist $\lambda$ nicht polynomial in der Frequenz $s$. Daher stellt der Operator $\Lambda$ mit $\mathcal{F}(\Lambda(v))=\lambda \mathcal{F} v$ einen nicht lokalen Operator dar, dessen explizite Form nicht bekannt ist und dessen numerische Auswertung aufwendig ist (entsprechend dem Lösen des Randproblems selbst, siehe [20]). Eine geeignete Approximation von $\Lambda$ durch lokale Operatoren erhält man jedoch durch polynomiale Approximation von $\lambda$ in $s$. Im Fall einer Approximation nullter und erster Ordnung ersetzt man dazu $\lambda$ durch

$$
\lambda_{0}:=p \quad \text { bzw. } \quad \lambda_{1}:=p+i q s
$$

mit noch zu bestimmenden Koeffizienten $p, q \in \mathbb{R}$. Dies führt auf die zugehörigen Randoperatoren

$$
\Lambda_{0}:=p \quad \text { bzw. } \quad \Lambda_{1}:=p+q \partial_{t} .
$$

Eine Approximation des Fourier-Symbols für kleine Frequenzen durch Taylor-Polynome wird in [54] im Fall eines Dirichlet-Neumann-Algorithmus für stationäre Konvektions-DiffusionsProbleme betrachtet. Dies entspricht im wesentlichen einer Taylor-Approximation von $\rho$ um $s=0$ :

$$
\frac{1}{2 \varepsilon} \sqrt{b^{2}+4 \varepsilon(c+\imath s)}=\frac{\sqrt{b^{2}+4 \varepsilon c}}{2 \varepsilon}+\frac{1}{\sqrt{b^{2}+4 \varepsilon c}} \imath s+O\left(s^{2}\right) .
$$

In der zitierten Arbeit werden überlappende Teilgebiete genutzt. Dies sorgt in der Konvergenzrate für einen Faktor, der große Frequenzen mit wachsener Überlappungsbreite exponentiell dämpft.

Die Nutzung von Taylor-Polynomen für kleine Frequenzen wird in [26] für zeitabhängige Konvektions-Diffusions-Reaktions-Probleme über eindimesionalen Gebieten wieder aufgegriffen, jedoch auch für nicht überlappende Teilgebiete untersucht. Zudem wird dort alternativ die Wahl der Koeffizienten mit Hilfe einer Optimierungsrechnung für die Konvergenzratenfunktion betrachtet, die sich insbesondere im Fall nicht überlappender Teilgebiete als überlegen erweist. Die Koeffizienten $p$ bzw. $p$ und $q$ werden numerisch so bestimmt, daß sie die Konvergenzrate minimieren.

Übertragen auf den hier untersuchten Dirichlet-Robin-Algorithmus mit Relaxationsparameter $\sigma$ bedeutet das bei Approximation nullter Ordnung, den Koeffizienten $p$ so zu wählen, daß er das Problem

$$
\min _{p} \max _{s}\left|1-\sigma\left(1+C_{\lambda_{0}}(s)\right)\right|=\min _{p} \max _{s}\left|1-\sigma\left(1+\frac{\varepsilon \rho \operatorname{coth}(\rho l)+p}{\varepsilon \rho \operatorname{coth}(\rho(1-l))-p}\right)\right|
$$

löst. Dabei ist zu berücksichtigen, daß durch die Diskretisierung des Problems nicht sämtliche Frequenzen $s \in \mathbb{R}$ aufgelöst werden. Vielmehr ist $\frac{\pi}{T}$ die minimale und $\frac{\pi}{\Delta t}$ die maximale 
Frequenz, die bei einem Zeitinvall $[0, T]$ und der Zeitschrittweite $\Delta t$ repräsentiert wird. Bei dem obigen Min-max-Problem ist also $\left[\frac{\pi}{T}, \frac{\pi}{\Delta t}\right]$ das für $|s|$ relevante Intervall.

Die Untersuchungen dieses Abschnitts lassen sich mit geringem Aufwand auf den zweidimensionalen Fall übertragen (vergl. [49]).

\subsection{Untersuchung des Dirichlet-Robin-Algorithmus im zwei- dimensionalen Fall}

Ausgangspunkt der folgenden zweidimensionalen Analyse sind Konvektions-DiffusionsReaktions-Probleme über dem Einheitsquadrat $\Omega:=(0,1) \times(0,1)$

$$
\left\{\begin{array}{l}
\partial_{t} u+L u=f \quad \text { in }(0, T) \times \Omega \\
u=0 \quad \text { auf }(0, T) \times \partial \Omega \\
u(0, x, y)=u_{0}(x, y)
\end{array}\right.
$$

mit

$$
L u=-\varepsilon u_{x x}-\varepsilon u_{y y}+b_{1} u_{x}+b_{2} u_{y}+c u
$$

und konstanten Koeffizienten $\varepsilon>0, b_{1}, b_{2}, c \geqslant 0$. Das Gebiet $\Omega$ wird zerlegt durch die zwei Teilgebiete $\Omega_{1}:=(0, l) \times(0,1)$ und $\Omega_{2}:=(l, 1) \times(0,1)$ für ein $l \in(0,1)$. Den künstlichen Rand $\Gamma$ bildet also die Menge $\{l\} \times(0,1)$, siehe dazu auch Abbildung 7.7.

Wiederum betrachten wir unseren Gebietszerlegungsalgorithmus (5.13) - (5.15) mit einem allgemeinen linearen Randoperator $\Lambda$ statt des $\gamma$-Terms der Robin-Randbedingung:

$$
\begin{aligned}
& \left\{\begin{array}{l}
\partial_{t} u_{1}^{k}+L u_{1}^{k}=f \quad \text { in }(0, T) \times \Omega_{1} \\
u_{1}^{k}=0 \quad \text { auf }(0, T) \times \partial \Omega_{1} \cap \partial \Omega \\
u_{1}^{k}(t, l, y)=g^{k-1}(t, y)
\end{array}\right. \\
& \left\{\begin{array}{l}
\partial_{t} u_{2}^{k}+L u_{2}^{k}=f \quad \text { in }(0, T) \times \Omega_{2} \\
u_{2}^{k}(t)=0 \quad \text { auf }(0, T) \times \partial \Omega_{2} \cap \partial \Omega \\
\varepsilon\left(u_{2}^{k}\right)_{x}-\frac{1}{2} b_{1} u_{2}^{k}+\Lambda\left(u_{2}^{k}\right)=\varepsilon\left(u_{1}^{k}\right)_{x}-\frac{1}{2} b_{1} u_{1}^{k}+\Lambda\left(u_{1}^{k}\right) \quad \text { auf }(0, T) \times \Gamma
\end{array}\right.
\end{aligned}
$$

mit den Anfangsbedingungen $u_{j}^{k}(0, x, y)=\left.u_{0}\right|_{\Omega_{i}}(x, y), j=1,2$, und der Relaxationsgleichung

$$
g^{k}:=\left.\sigma u_{2}^{k}\right|_{\Gamma}+(1-\sigma) g^{k-1} .
$$

Auch hier bezeichne $\left(\left(\tilde{u}_{1}^{k}, \tilde{u}_{2}^{k}\right)\right)_{k \in \mathbb{N}}$ die Differenzenfolge mit $\tilde{u}_{j}^{k}:=u_{j}^{k}-\left.u\right|_{\Omega_{j}}, j=1,2$, und es sei $\tilde{g}^{k-1}=\left.\tilde{u}_{1}^{k}\right|_{\Gamma}, k \geqslant 1$. 
Im vorliegenden zweidimensionalen Fall bezeichne $\mathcal{F}$ die Fourier-Transformation bezüglich der Zeit $t$ und der Raumvariablen $y$ gemäß

$$
\mathcal{F} v(s, x, z)=\frac{1}{\sqrt{2 \pi}} \int_{\mathbb{R}} \int_{\mathbb{R}} e^{-\imath(s t+y z)} v(t, x, y) d y d t
$$

Es ist $s$ also die Fourier-Variable zu $t, z$ die zu $y$. Um $\mathcal{F}$ auf $\tilde{u}_{1}^{k}, \tilde{u}_{2}^{k}$ anwenden zu können, seien diese sowohl für $t<0$ als auch für $|y|>1$ fortgesetzt durch $\tilde{u}_{1}^{k}(t, x, y)=\tilde{u}_{2}^{k}(t, x, y)=0$. Analog zum eindimensionalen Fall ergeben sich dann die zwei gewöhnlichen Differentialgleichungen

$$
\varepsilon\left(w_{j}^{k}\right)_{x x}-b_{1}\left(w_{j}^{k}\right)_{x}-\left(c+\imath\left(s+b_{2} z\right)\right) w_{j}^{k}=0, \quad j=1,2,
$$

für $w_{j}^{k}(s, x, z):=\mathcal{F} \tilde{u}_{j}^{k}(s, x, z)$ in $x$, die allgemein erfüllt werden durch

$$
w_{j}^{k}(s, x, z)=A_{j}^{k}(s, z) e^{(\omega+\rho) x}+B_{j}^{k}(s, z) e^{(\omega-\rho) x}
$$

mit den Koeffizientenfunktionen $A_{j}^{k}, B_{j}^{k}$. Dabei ist

$$
\omega:=\frac{b_{1}}{2 \varepsilon}, \quad \rho=\rho(s, z):=\frac{1}{2 \varepsilon} \sqrt{b_{1}^{2}+4 \varepsilon c+4 \varepsilon^{2} z^{2}+\imath 4 \varepsilon\left(s+b_{2} z\right)} .
$$

Wie im Beweis von Lemma 8.1.1 zeigt man

Lemma 8.2.1 Der Algorithmus (8.13) - (8.15) mit $T=\infty$ generiert in zwei Iterationen die globale Lösung von (8.12), d. $h . u_{1}^{k}=\left.u\right|_{\Omega_{1}}$ und $u_{2}^{k}=\left.u\right|_{\Omega_{2}}$ für $k \geqslant 2$, falls $\Lambda$ der lineare Operator mit dem zugehörigen Fourier-Symbol

$$
\lambda=\varepsilon \rho((1-\sigma) \operatorname{coth}(\rho(l-1))-\sigma \operatorname{coth}(\rho l))
$$

ist, wobei

$$
\rho=\rho(s, z)=\frac{1}{2 \varepsilon} \sqrt{b_{1}^{2}+4 \varepsilon c+4 \varepsilon^{2} z^{2}+\imath 4 \varepsilon\left(s+b_{2} z\right)} .
$$

Analog zu (8.8) ergibt sich die Relaxationsgleichung für $\mathcal{F} \tilde{g}^{k} \mathrm{zu}$

$$
\mathcal{F} \tilde{g}^{k}(s, z)=\left(1-\sigma\left(1+C_{\lambda}(s, z)\right)\right) \mathcal{F} \tilde{g}^{k-1}(s, z),
$$

mit

$$
C_{\lambda}(s, z):=\frac{\varepsilon \rho(s, z) \operatorname{coth}(\rho(s, z) l)+\lambda}{\varepsilon \rho(s, z) \operatorname{coth}(\rho(s, z)(1-l))-\lambda} .
$$

Eine polynomiale Approximation nullter und erster Ordnung von $\lambda$ der Form

$$
\lambda_{0}:=p \quad \text { bzw. } \quad \lambda_{1}:=p+\imath q\left(s+b_{2} z\right)
$$

mit Koeffizient $p \in \mathbb{R}$ und dem für $\imath s$ und $\imath b_{2} z$ gemeinsamen Koeffizient $q \in \mathbb{R}$ (vergl. [49]) führt auf die jeweiligen lokalen Randoperatoren

$$
\Lambda_{0}:=p \quad \text { bzw. } \quad \Lambda_{1}:=p+q \partial_{t}+q b_{2} \partial_{y} .
$$


Die Approximation nullter Ordnung und der zugehörige Randoperator $\Lambda_{0}$ bedeutet am Austauschrand eine übliche Robin-Randbedingung. Dies wird also durch die in Kapitel 6 geführte Konvergenzanalyse abgedeckt. Um eine optimale Konvergenzrate bei Approximation nullter Ordnung zu erreichen, wählen wir $p=p_{\min }$ als Lösung des Min-max-Problems

$$
\min _{p} \max _{s}\left|1-\sigma\left(1+C_{\lambda_{0}}(s, z)\right)\right|=\min _{p} \max _{s, z}\left|1-\sigma\left(1+\frac{\varepsilon \rho \operatorname{coth}(\rho l)+p}{\varepsilon \rho \operatorname{coth}(\rho(1-l))-p}\right)\right| .
$$

Dabei sind die für die Frequenzen $s$ und $z$ relevanten Intervalle wiederum durch die minimal und maximal auflösbaren Frequenzen bestimmt, also $|s| \in\left[\frac{\pi}{T}, \frac{\pi}{\Delta t}\right]$ und $|z| \in\left[\frac{\pi}{l}, \frac{\pi}{h}\right]$ (Vergl. 8.11 und nachfolgende Anmerkungen sowie [49]).

Wir haben in unsere Analyse die Teilgebietsweite $l$ (bzw. $1-l$ ) mit einbezogen, indem wir beschränkte Gebiete betrachten. Daraus resultieren die Faktoren $\operatorname{coth}(\rho l)$ und $\operatorname{coth}(\rho(1-l))$. Solange $\frac{b}{\varepsilon}$ groß gegenüber $l$ bleibt, spielt die Größe von $l$, also das Verhältnis von $l$ zu $l-1$ keine entscheidende Rolle für die Konvergenz. Dies war bereits an den numerischen Resultaten aus Beispiel 7.4.3 zu beobachten.

Betrachtet man hingegen den Dirichlet-Robin-Algorithmus für die unbeschränkten Gebiete $\Omega_{1}=\mathbb{R}^{<0} \times(0,1), \Omega_{2}=\mathbb{R}^{>0} \times(0,1)$ oder $\Omega_{1}=\mathbb{R}^{<0} \times \mathbb{R}, \Omega_{2}=\mathbb{R}^{>0} \times \mathbb{R}$, so gelangt man durch ähnliches Vorgehen wie in Lemma 8.1.1 bzw. Lemma 8.2.1 speziell im Fall ohne Relaxation $(\sigma=1)$ zu der Konvergenzratenfunktion

$$
\left|C_{\lambda_{0 / 1}}(s)\right|=\left|\frac{\frac{1}{2} \sqrt{b^{2}+4 \varepsilon(c+\imath s)}+\lambda_{0 / 1}}{\frac{1}{2} \sqrt{b^{2}+4 \varepsilon(c+\imath s)}-\lambda_{0 / 1}}\right|
$$

im eindimensionalen Fall und

$$
\left|C_{\lambda_{0 / 1}}(s, z)\right|=\left|\frac{\frac{1}{2} \sqrt{b_{1}^{2}+4 \varepsilon c+4 \varepsilon^{2} z^{2}+\imath 4 \varepsilon\left(s+b_{2} z\right)}+\lambda_{0 / 1}}{\frac{1}{2} \sqrt{b_{1}^{2}+4 \varepsilon c+4 \varepsilon^{2} z^{2}+\imath 4 \varepsilon\left(s+b_{2} z\right)}-\lambda_{0 / 1}}\right|
$$

im zweidimensionalen Fall. Interessanterweise stimmen diese mit den Konvergenzratenfunktionen überein, die man erhält, wenn man optimierte Randbedingungen für beide Teilgebiete heranzieht (siehe [28], Abschnitt 5.2 für eindimensionale Probleme, [49], Abschnitt 8.1 für zweidimensionale Probleme).

\subsection{Numerische Ergebnisse}

Nachdem wir durch die Analysis zu Konvektions-Diffusions-Reaktions-Problemen mit konstanten Koeffizienten über rechteckigen Teilgebieten eine Möglichkeit vorliegen haben, die Austauschrandbedingungen unseres Gebietszerlegungsverfahrens zu optimieren, sollen nun die Auswirkungen dieser Optimierung numerisch dargestellt werden.

Wir beziehen uns auf dieselben zweidimensionalen Probleme, wie sie eingangs im Abschnitt 7.4 angegeben wurden und nutzen weiterhin die Bezeichnungen aus Tabelle 7.1. Auch bei der räumlichen und zeitlichen Diskretiesierung gehen wir wie dort beschrieben vor. Auf die Abhängigkeit von den Diskretisierungsparametern $h$ und $\Delta t$ wird eingegangen. 
Beispiel 8.3.1 (Optimierte Randbedingungen) In diesem Beispiel betrachten wir die Konvergenzgeschwindigkeit des Dirichlet-Robin-Algorithmus in Abhängigkeit von der Wahl von $\sigma$ sowie $\gamma$ und vergleichen eine Reihe von numerischen Ergebnissen mit Berechnungen zu optimalen Austauschrandbedingungen auf der Grundlage des vorausgegangenen Abschnitts 8.2 .

Dargestellt sind Ergebnisse für die Fälle $\left(A_{1}\right),\left(A_{10^{-4}}\right)$ und $\left(B_{10^{-4}}\right)$ jeweils ohne Reaktionsterm $(c=0)$ über dem Gebiet $\Omega:=(0,1) \times(0,1)$. Die Zerlegung von $\Omega$ durch zwei Teilgebiete erfolgt mit $l=\frac{1}{2}$ wie im Abschnitt 8.2 angegeben. Die Diskretisierung geschieht für beide Teilgebiete mit dem Gitterparameter $h=0.025$ und der Zeitschrittweite $\Delta t=0.05$.

Um die Auswirkungen durch Variation über die Anzahl der Zeitschritte bei der Berechnung der diskreten Norm in der Zeit gering zu halten, betrachten wir den relativen Fehler

$$
r_{2}^{k}:=\left(\sum_{i=1,2} \frac{\left\|u_{i, h}^{k-1}-u_{i, h}^{k}\right\|_{L^{2}\left(0, T ; L^{2}\left(\Omega_{i}\right)\right)}^{2}}{\left\|u_{i, h}^{k}\right\|_{L^{2}\left(0, T ; L^{2}\left(\Omega_{i}\right)\right)}^{2}}\right)^{\frac{1}{2}}
$$

$\mathrm{Zu}$ Beginn geben wir die Konvergenzgeschwindigkeit bei Variation des Relaxationsparameters $\sigma$ global über das Intervall $(0,1]$ an. In Konturdiagrammen für die Fälle $\left(A_{1}\right)$ (Abbildung 8.2) und $\left(A_{10^{-4}}\right)$ (Abbildung 8.3) sind zum einen die Anzahlen der Iterationen abgetragen, die der Dirichlet-Robin-Algorithmus benötigt, um $r_{2}^{k}<10^{-12}$ zu erreichen. Im ersten Fall variiert $\gamma \in \mathbb{R}$ dabei über $[0,45]$, im zweiten Fall über $[0,3]$. Zum anderen sind für $(\sigma, p) \in(0,1] \times[0,45]$ bzw. $(\sigma, p) \in(0,1] \times[0,3]$ die Maxima

$$
\max _{s, z}\left|1-\sigma\left(1+C_{\lambda_{0}}(s, z)\right)\right|, \quad|s| \in\left[\frac{\pi}{T}, \frac{\pi}{\Delta t}\right],|z| \in\left[\frac{\pi}{l}, \frac{\pi}{h}\right]
$$

dargestellt.

Für $\sigma>\frac{1}{2}$ liegen die Werte für $\gamma$, die die Konvergenzratenfunktion bzw. die Iterationszahlen des Dirichlet-Robin-Algorithmus minimieren, dicht beieinander. Dies bestätigt numerisch die Eignung der Analysis aus Abschnitt 8.2 zur Bestimmung optimierter Randbedingungen. Die Minima sind in jeweils beiden Konturdiagrammen mit einem + gekennzeichnet. Auch global bildet das Konturdiagramm der Konvergenzratenfunktion $1-\sigma\left(1+C_{\lambda}(s, z)\right)$ das Konvergenzverhalten des Algorithmus gut ab. Allerdings mußte im Fall $\left(A_{10^{-4}}\right)$ der Koeffizient $p$ mit dem Faktor 3 skaliert werden. Diese Diskrepanz zwischen dem optimalen $\gamma$ und $p$ bedarf als offene Frage noch weiterer Klärung.

Im Abschnitt 8.1 wurde bereits deutlich, daß für $\sigma=\frac{1}{2}$ der Nulloperator der (exakte) optimale Randoperator ist. Entsprechend zeigen die Konturdiagramme der Konvergenzratenfunktion das globale Minimum bei $\left(\frac{1}{2}, 0\right)$. Dies wird jedoch durch die Rechnungen mit dem DirichletRobin-Algorithmus nicht wiedergegeben. Hier werden die minimalen Iterationszahlen für $\sigma>$ $\frac{1}{2}$ erreicht. 

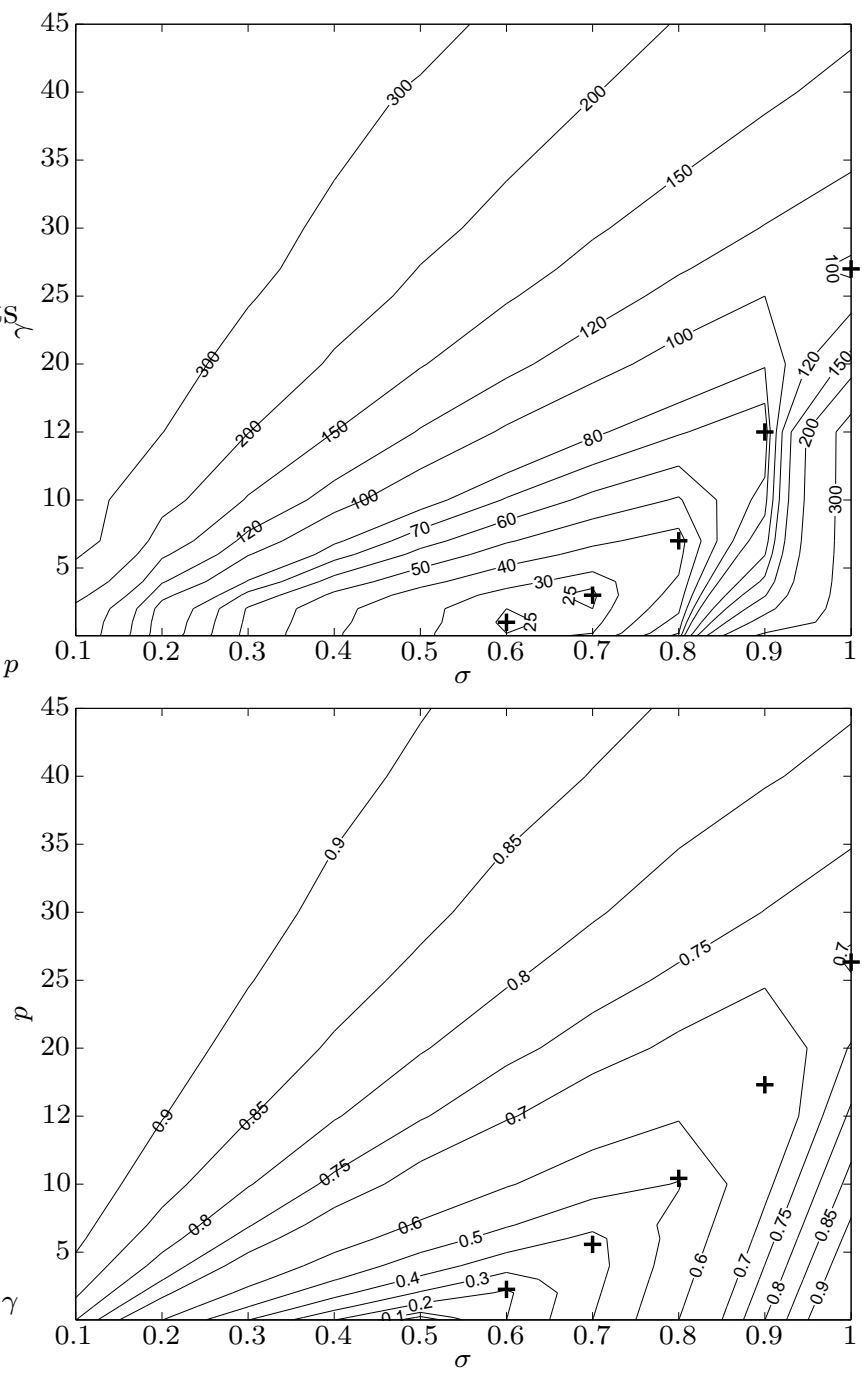

Abbildung 8.2: Anzahl der Iterationen zur Erreichung $r_{2}^{k}<10^{-12}$ (oben) und Maxima der Konvergenzratenfunktion $\left|1-\sigma\left(1+C_{\lambda_{0}}(s, z)\right)\right|$ in Abhängigkeit von $\sigma$ und $\gamma$ für den Fall $\left(A_{1}\right)$. 

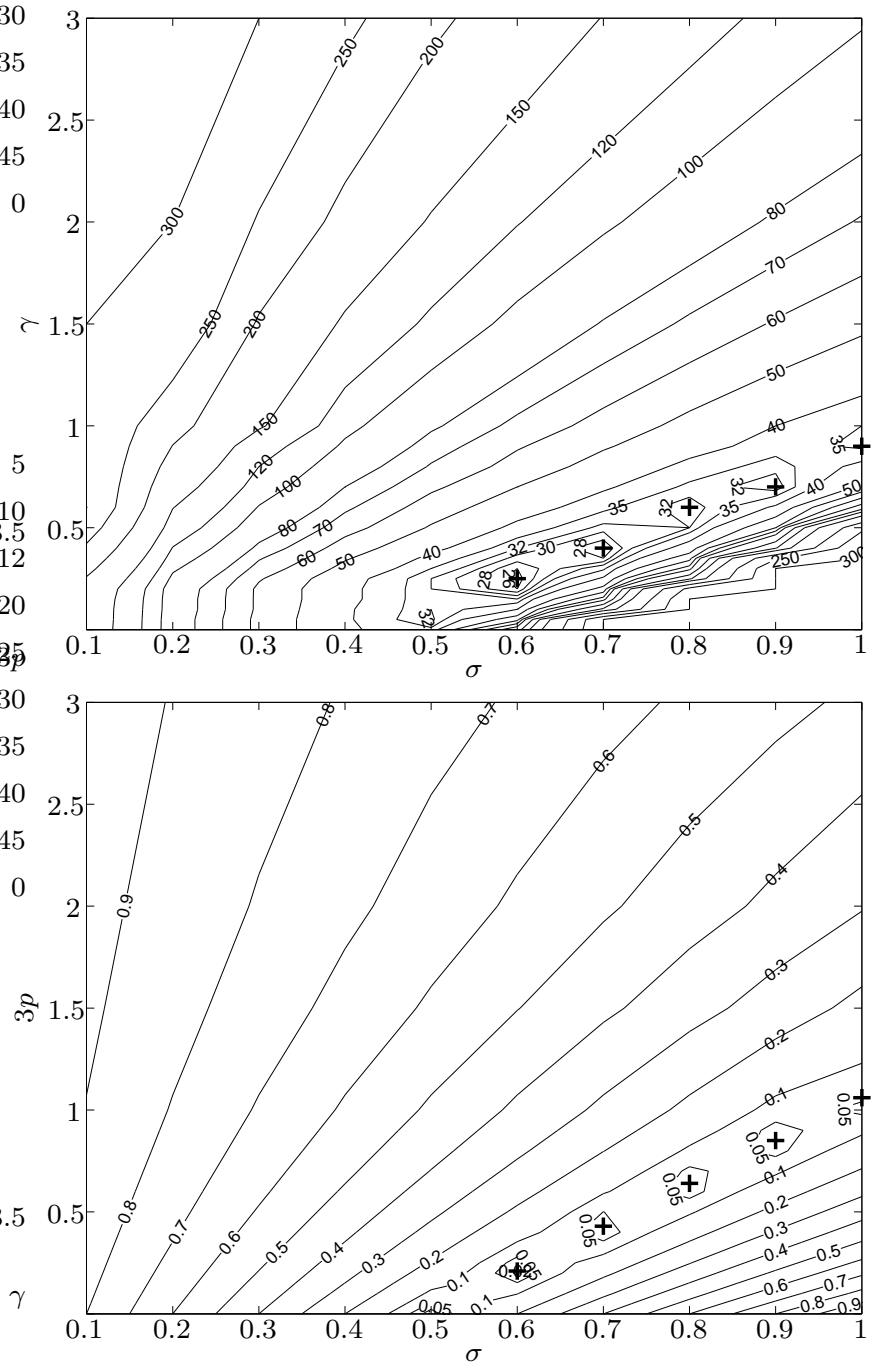

Abbildung 8.3: Anzahl der Iterationen zur Erreichung $r_{2}^{k}<10^{-12}$ (oben) und Maxima der Konvergenzratenfunktion $\left|1-\sigma\left(1+C_{\lambda_{0}}(s, z)\right)\right|$ in Abhängigkeit von $\sigma$ und $\gamma$ für den Fall $\left(A_{10^{-4}}\right)$

Zur detaillierten Betrachtung der Konvergenzverläufe greifen wir als nächstes einzelne Werte für $\sigma$ heraus, nämlich $\sigma=1,0.6,0.5$. Die Abbildungen 8.4 zeigt für $\left(A_{1}\right)$ die Iterationszahlen, die zur Unterschreitung der Fehlerschranke $10^{-12}$ benötigt werden, in Abhängigkeit von $\gamma$ für die Zeitschrittweiten $\Delta t=\frac{1}{10}, \frac{1}{20}, \frac{1}{40}, \frac{1}{100}$ und stellt die entsprechenden Verläufe der Konvergenzratenfunktion gegenüber. Weiterhin ist $h=0.025$. Abbildung 8.5 zeigt dasselbe für $\left(A_{10^{-4}}\right)$.

Im Fall $\left(A_{10^{-4}}\right)$ ohne Relaxation $(\sigma=1)$ erkennt man eine Aufsplittung nach den unterschiedlichen Zeitschrittweiten, die Verschiebung des optimalen Randparameters und der minimalen Iterationszahlen. Auch die Analysis zeigt ein solches Verhalten, jedoch viel schwächer ausgeprägt, und die optimalen Werte für den Randparameter werden hier nicht exakt getroffen.

In allen anderen Fällen liegen die Resultate für sämtliche Zeitschrittweiten übereinander, zeigt sich also keine $\Delta t$-Abhängigkeit. Die kleinsten Iterationszahlen werden für $\sigma=0.6$ erreicht. 
Hier werden die optimalen Werte für $\gamma$ durch die Analysis sehr gut prognostiziert. Wie bereits erwähnt, findet sich die Optimalität des Nulloperators für $\sigma=0.5$ nicht in den Rechnungen mit dem Algorithmus. Es bildet sich vielmehr ein die Null einschließendes Intervall von $\gamma$ Werten mit minimaler Iterationszahl.
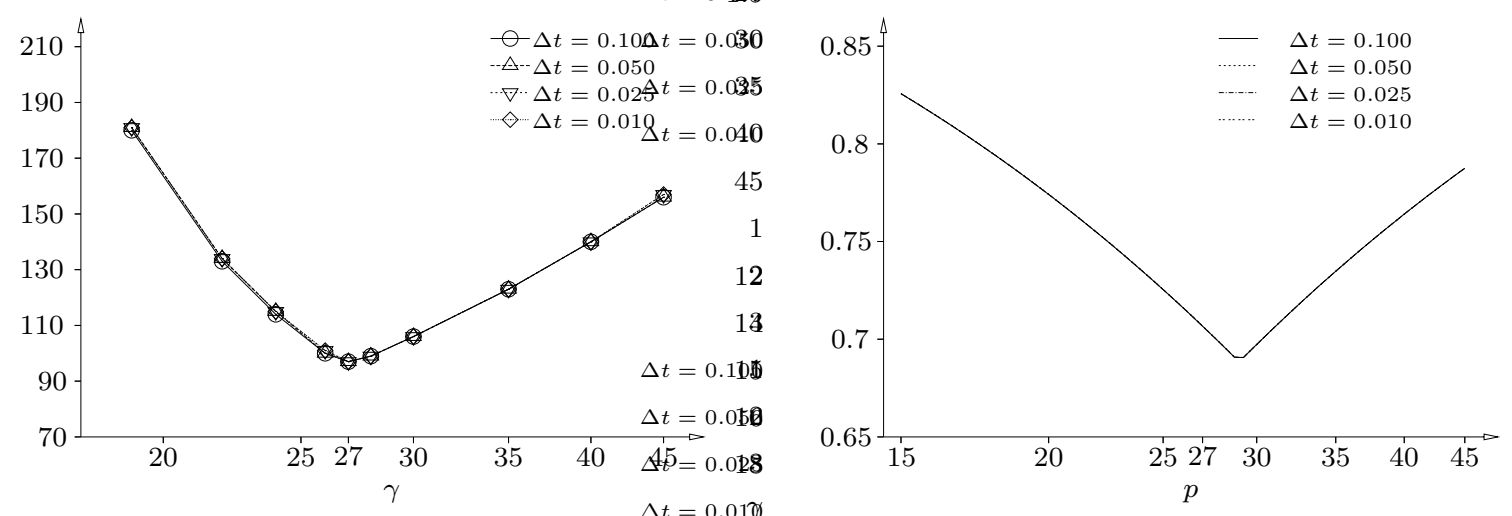

$$
\sigma=1.0
$$
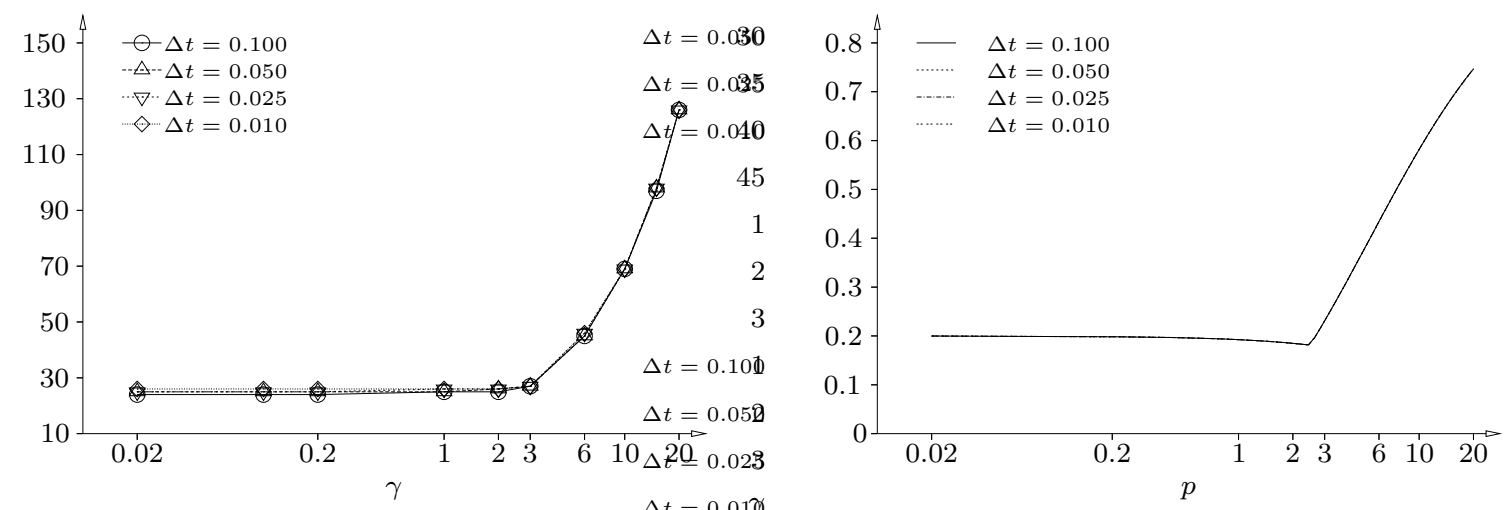

$\sigma=0.6$
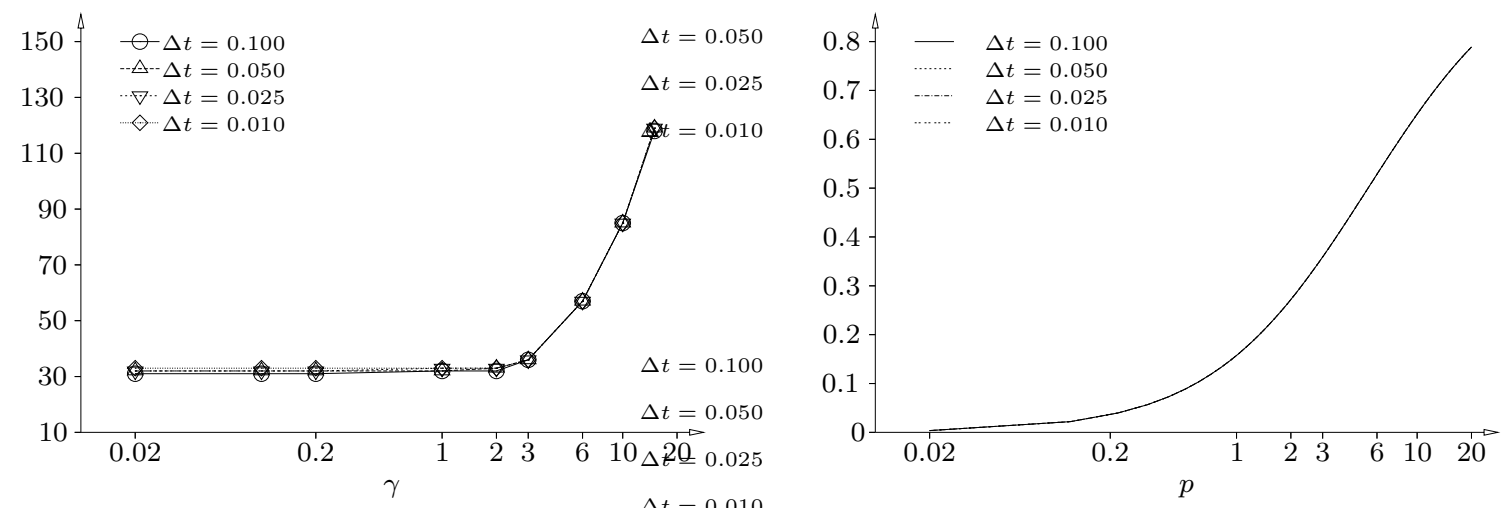

$$
\sigma=0.5
$$

Abbildung 8.4: Mindestanzahl der DD-Iterationen, die nötig sind, um $r_{2}^{k}<10^{-12}$ zu erreichen, und Maxima der Konvergenzratenfunktion $\left|1-\sigma\left(1+C_{\lambda_{0}}(s, z)\right)\right|$ in Abhängigkeit der Wahl von $\gamma$ bzw. $p$ für unterschiedliche Zeitschrittweiten $\Delta t$, Fall $\left(A_{1}\right)$. 

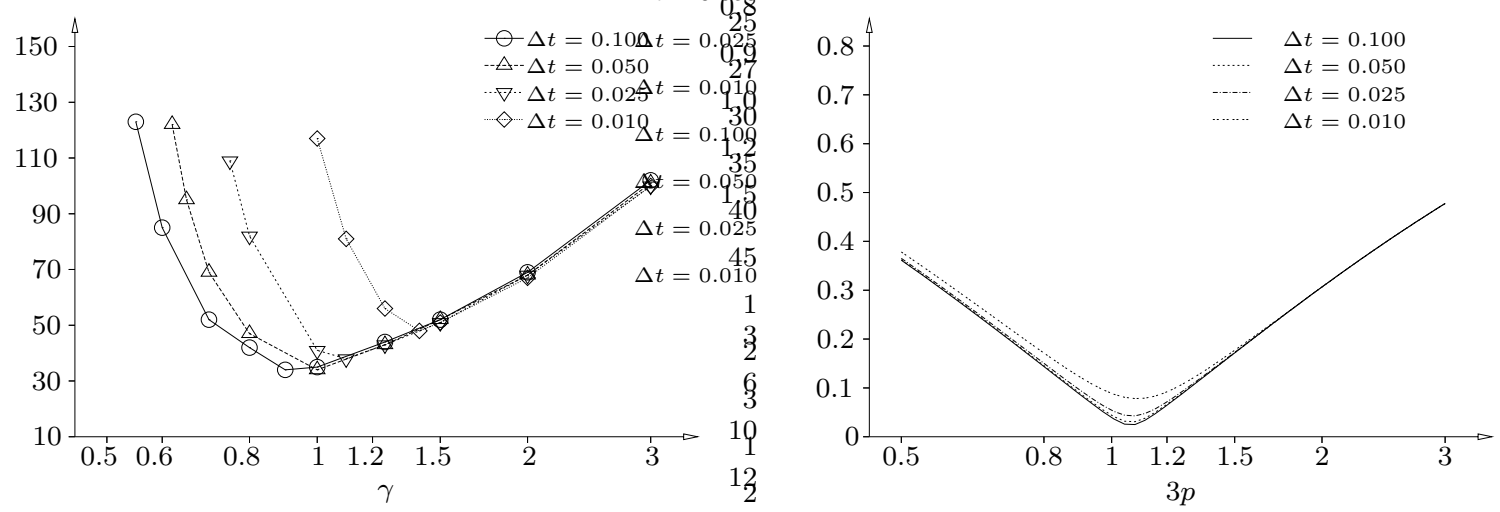

$\sigma=1.0$
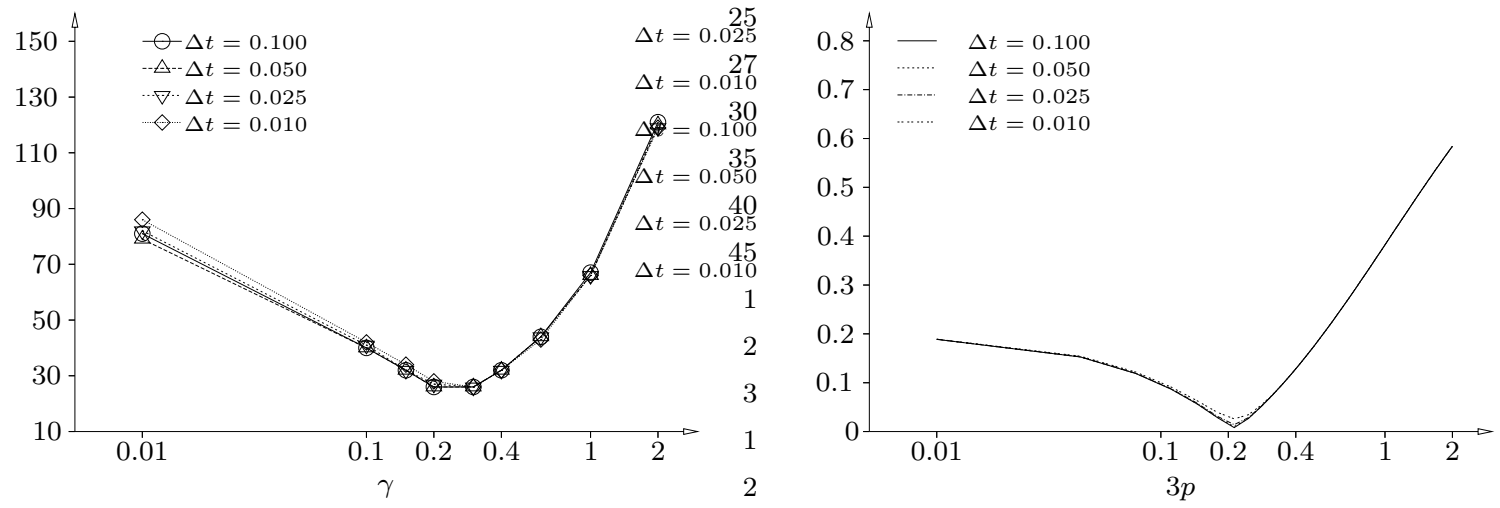

$\sigma=0.6$
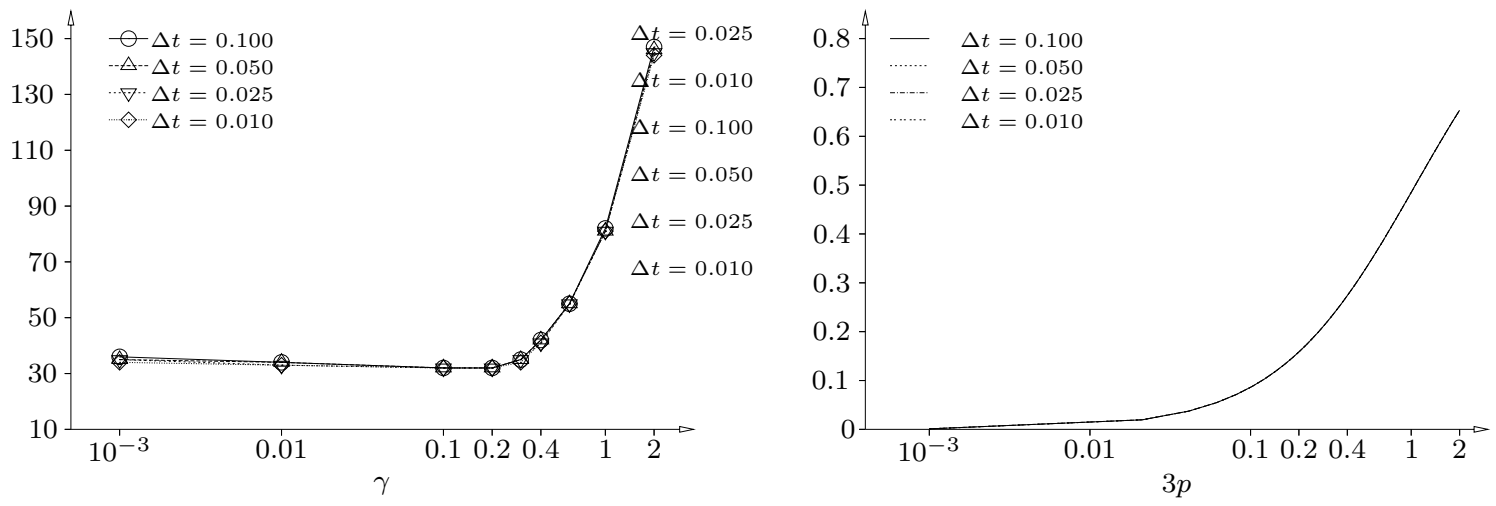

$\sigma=0.5$

Abbildung 8.5: Mindestanzahl der DD-Iterationen, die nötig sind, um $r_{2}^{k}<10^{-12}$ zu erreichen, und Maxima der Konvergenzratenfunktion $\left|1-\sigma\left(1+C_{\lambda_{0}}(s, z)\right)\right|$ in Abhängigkeit der Wahl von $\gamma$ bzw. $p$ für unterschiedliche Zeitschrittweiten $\Delta t$, Fall $\left(A_{10^{-4}}\right)$. 

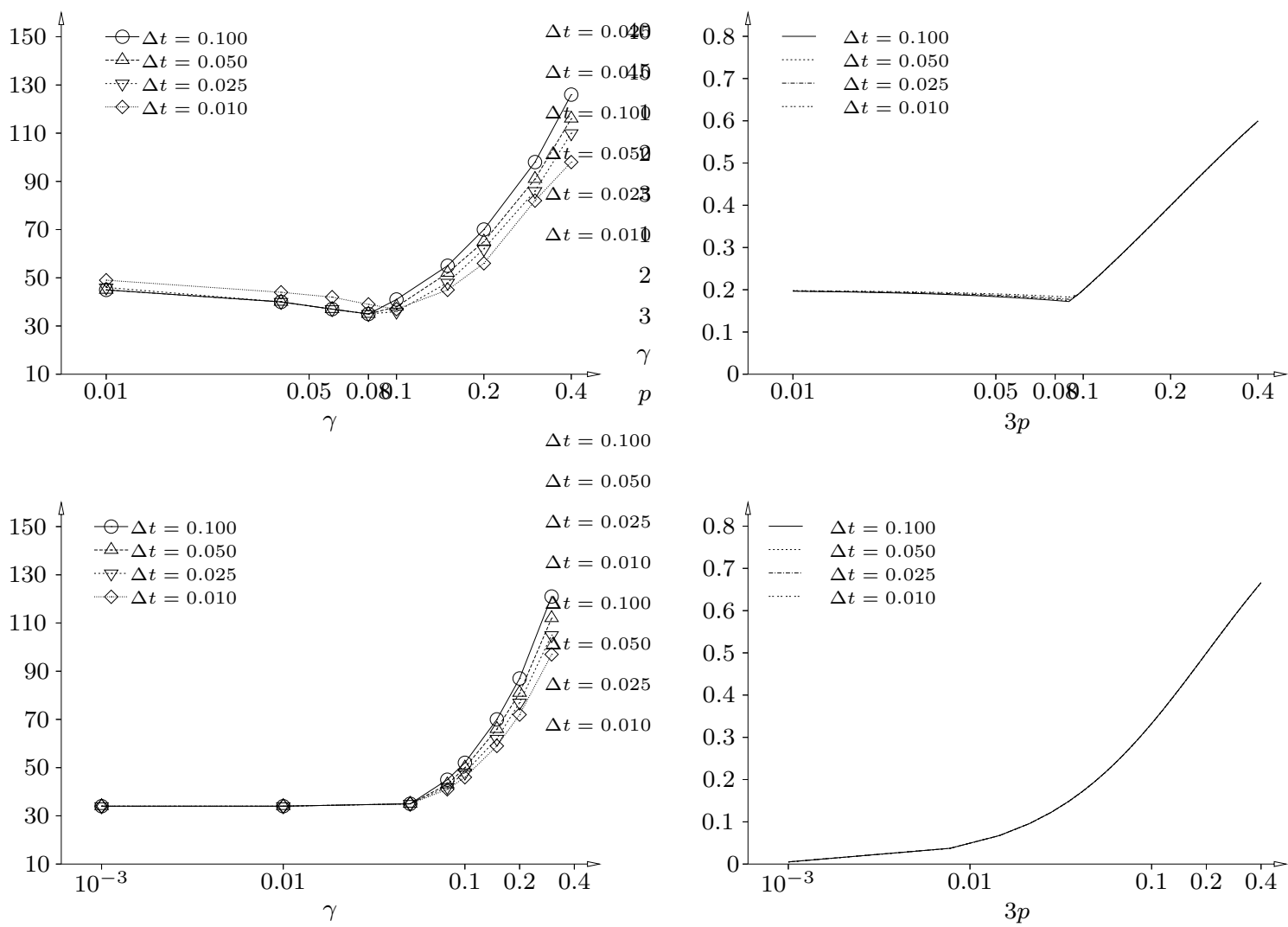

Abbildung 8.6: Mindestanzahl der DD-Iterationen, die nötig sind, um $r_{2}^{k}<10^{-12}$ zu erreichen, und Maxima der Konvergenzratenfunktion $\left|1-\sigma\left(1+C_{\lambda_{0}}(s, z)\right)\right|$ in Abhängigkeit der Wahl von $\gamma$ bzw. $p$ für unterschiedliche Zeitschrittweiten $\Delta t$, Fall $\left(B_{10^{-4}}\right)$.

Im Fall $\left(B_{10^{-4}}\right)$ bestätigen sich die Übereinstimmung von analytisch prognostizierten und numerisch ermittelten minimalen Iterationszahlen für Werte $\sigma \in(0.5,1)$ außer in der Nähe von $\sigma=0.5$, wo die Analysis die Optimalität des Nulloperators erwarten lässt. Abbildung 8.6 gibt die Kurven für $\sigma=0.6$ und $\sigma=0.5$ wieder. Die geringste Iterationszahl wird wiederum mit $\sigma=0.6$ erreicht, hier stellt sich allerdings diese Iterationszahl auch mit $\sigma=0.5$ ein.

Bei diesem deutlich kritischeren Verhähltnis von Konvektionsrichtung zu Austauschrand (Parallelströmung) zeigt sich oberhalb des optimalen Wertes für $\gamma$ eine geringe $\Delta t$-Abhängigkeit.

\section{Beispiel 8.3.2 (Einfluß der Gitterweite auf die Konvergenz bei optimiertem $\gamma$ )}

Wir greifen nun Beispiel 7.4.2 wieder auf, und zeigen, daß sich bei optimal gewähltem Austauschrandparameter $\gamma$ auch im konvektionsdominanten Fall Unabhängigkeit der Konvergenzgeschwindigkeit vom Gitterparameter $h$ ergibt. Tabelle 8.1 belegt dies durch numerische Ergebnisse für $\left(A_{10^{-4}}\right)$ und $\left(B_{10^{-4}}\right)$, während Tabelle 7.2 noch eine deutliche Abhängigkeit von $h$ zeigte. Es liegen dieselben Rechnungen wie in Beispiel 7.4.2 zugrunde, jedoch mit Werten für $\sigma$ und $\gamma$, wie sie im Beispiel 8.3.1 zur Erlangung minimaler Iterationszahlen angegeben wurden. 


\begin{tabular}{|c|c|c|c|c|c|c|c|}
\hline \multirow{2}{*}{ Fall } & \multirow{2}{*}{$\sigma$} & \multirow[b]{2}{*}{$\gamma$} & \multirow{2}{*}{$h$} & \multicolumn{4}{|c|}{ Anzahl Iterationen zur Erreichung $e_{2}^{k}<$} \\
\hline & & & & $10^{-4}$ & $10^{-6}$ & $10^{-8}$ & $10^{-10}$ \\
\hline \multirow{5}{*}{$\left(A_{10^{-4}}\right)$} & \multirow{5}{*}{0.6} & \multirow{5}{*}{0.25} & 0.16 & 9 & 14 & 19 & 24 \\
\hline & & & 0.08 & 11 & 15 & 19 & 24 \\
\hline & & & 0.04 & 9 & 13 & 18 & 23 \\
\hline & & & 0.02 & 9 & 13 & 18 & 23 \\
\hline & & & 0.01 & 10 & 15 & 20 & 25 \\
\hline \multirow{5}{*}{$\left(B_{10^{-4}}\right)$} & \multirow{5}{*}{0.5} & \multirow{5}{*}{0.02} & 0.16 & 11 & 17 & 23 & 29 \\
\hline & & & 0.08 & 10 & 17 & 23 & 29 \\
\hline & & & 0.04 & 10 & 16 & 22 & 29 \\
\hline & & & 0.02 & 9 & 16 & 22 & 29 \\
\hline & & & 0.01 & 12 & 17 & 22 & 30 \\
\hline
\end{tabular}

Tabelle 8.1: Äbhängigkeit der Konvergenzgeschwindigkeit von der Gitterweite für die Fälle $\left(A_{10^{-4}}\right)$ und $\left(B_{10^{-4}}\right)$ bei optimal gewähltem $\gamma$

\section{Beispiel 8.3.3 (Einfluß des Austauschrand-Startwerts auf die Konvergenz)}

Anhand der Fälle $\left(A_{\varepsilon}\right), \varepsilon=1,10^{-4}, 10^{-6}$, zeigen wir, daß die Wahl des Startwertes $g^{0}$ auf dem Austauschrand nicht von wesentlichem Einfluss auf den Konvergenzverlauf des DirichletRobin-Algorithmus ist. Wir führen dieselben Rechnungen für unterschiedliche Gitterweiten wie in den entsprechenden Fällen aus den Beispielen 7.4.2 und 8.3.2 durch. Während

\begin{tabular}{|c|c|c|c|c|c|c|c|}
\hline \multirow{2}{*}{ Fall } & \multirow[b]{2}{*}{$\sigma$} & \multirow[b]{2}{*}{$\gamma$} & \multirow{2}{*}{$h$} & \multicolumn{4}{|c|}{ Anzahl Iterationen zur Erreichung $e_{2}^{k}<$} \\
\hline & & & & $10^{-4}$ & $10^{-6}$ & $10^{-8}$ & $10^{-10}$ \\
\hline \multirow{5}{*}{$\left(A_{1}\right)$} & \multirow{5}{*}{0.7} & \multirow{5}{*}{0.0} & 0.16 & 10 & 15 & 20 & 24 \\
\hline & & & 0.08 & 11 & 16 & 22 & 27 \\
\hline & & & 0.04 & 10 & 16 & 22 & 28 \\
\hline & & & 0.02 & 10 & 15 & 21 & 27 \\
\hline & & & 0.01 & 10 & 15 & 21 & 27 \\
\hline \multirow{5}{*}{$\left(A_{10^{-4}}\right)$} & \multirow{5}{*}{0.6} & \multirow{5}{*}{0.25} & 0.16 & 9 & 14 & 19 & 24 \\
\hline & & & 0.08 & 9 & 14 & 20 & 25 \\
\hline & & & 0.04 & 10 & 13 & 18 & 23 \\
\hline & & & 0.02 & 10 & 14 & 18 & 22 \\
\hline & & & 0.01 & 11 & 14 & 18 & 22 \\
\hline \multirow{5}{*}{$\left(A_{10^{-6}}\right)$} & \multirow{5}{*}{0.6} & \multirow{5}{*}{0.25} & 0.16 & 8 & 13 & 18 & 23 \\
\hline & & & 0.08 & 9 & 14 & 19 & 25 \\
\hline & & & 0.04 & 10 & 13 & 17 & 22 \\
\hline & & & 0.02 & 10 & 14 & 18 & 22 \\
\hline & & & 0.01 & 10 & 14 & 18 & 22 \\
\hline
\end{tabular}

Tabelle 8.2: Äbhängigkeit der Konvergenzgeschwindigkeit von der Gitterweite für die Fälle $\left(A_{\varepsilon}\right), \varepsilon=1,10^{-4}$, bei zufällig gewähltem Startwert $g^{0}$ 
jedoch dort durchweg mit dem Startwert $g^{0}=0$ gerechnet wurde, belegen wir in diesem Beispiel für jede einzelne Rechnug $g^{0}$ in jedem Austauschrandpunkt mit zufällig gewählten Werten. Die Ergebnisse gibt die Tabelle 8.2 wieder.

Über das Spektrum der Gitterweite $h$ sowie im Vergleich zu den entsprechenden Ergebnissen in Tabelle 7.2 bzw. Tabelle 8.1 zeigen die Konvergenzverläufe keine Abhängigkeit von der Wahl von $g^{0}$. Man beachte, daß im Fall $\left(A_{1}\right)$ nicht mit optimierter Randbedingung gerechnet wurde, um die Vergleichbarkeit zu den Daten in Beispiel 7.4.2 herzustellen.

Beispiel 8.3.4 (Nicht konstante Konvektion) Von besonderer Relevanz für Anwendungen sind die Fälle mit nicht konstantem Konvektionsvektor, die wir in diesem Beispiel untersuchen. Wir kehren dazu zu Beispiel 7.4.1 zurück und betrachten die Fälle $\left(E_{10^{-4}}\right)$ und $\left(E_{10^{-6}}\right)$, bei denen der Konvektionsvektor durch $b=(-y, x)^{T}$ gegeben ist, erneut. Dort wurden Rechnungen mit dem Dirichlet-Robin-Algorithmus präsentiert, die mit konstantem $\gamma \in \mathbb{R}$ durchgeführt wurden. Dabei zeigte sich eine sehr unbefriedigende Konvergenzgeschwindigkeit und eine deutliche Abhängigkeit von der Stärke $\varepsilon$ der Diffusion.

Hier sollen die Ergebnisse aus den vorangegangenen Abschnitten zur optimalen Wahl von $\gamma$ im Fall von konstanten Koeffizienten auch auf Fälle $\left(E_{10^{-4}}\right)$ und $\left(E_{10^{-6}}\right)$ mit nicht konstantem Konvektionsvektor angewendet werden. Sind $b_{1}:=-y$ und $b_{2}:=x$ die Komponenten der Konvektion in jedem Punkt $(x, y)$ auf dem Austauschrand $\Gamma$, so erhalten wir mit $\rho=\frac{1}{2 \varepsilon} \sqrt{b_{1}^{2}+4 \varepsilon c+4 \varepsilon^{2} z^{2}+\imath 4 \varepsilon\left(s+b_{2} z\right)}$ (vergl. Lemma 8.2.1) eine Funktion $\gamma=\gamma_{\text {opt }}$, die je$\operatorname{dem}(x, y) \in \Gamma$ gemäß des Min-max-Problems (8.16) einen optimierten Koeffizienten $p=p_{\text {opt }}$ zuordnet. Abbildung 8.7 zeigt $b$ in einzelnen Punkten auf dem Austauschrand. Wir bringen diese Funktion $\gamma=\gamma_{\text {opt }}$ bei unserem Gebietszerlegungsalgorithmus (5.13) - (5.15) zum Einsatz. Ein solches Vorgehen wird durch die Konvergenzuntersuchungen aus Kapitel 6 bzw. 7 abgedeckt, da dort auf dem Austauschrand Funktionen $\gamma \in L^{\infty}(\Gamma)$ zugelassen wurden.

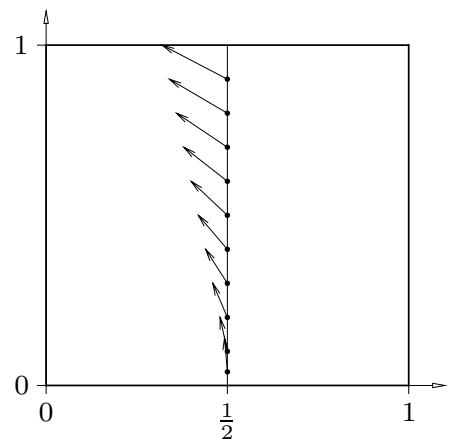

Abbildung 8.7: Konvektionsvektor $b$ in einzelnen Punkten auf dem Austauschrand

Die Abbildung 8.8 zeigt abgetragen über der Anzahl der Iterationen im Dirichlet-RobinAlgorithmus die Entwicklung der Fehler $e_{0}^{k}, e_{\infty, \Gamma}^{k}$ und $e_{\varepsilon}^{k}$ (vergl. (7.26)). Den Ergebnissen liegen dieselben Rechnungen zugrunde, die auch in Beispiel 7.4.1 durchgeführt wurden. Allein die Wahl des Austauschrandparameters $\gamma$ wurde wie oben beschrieben angepaßt. Damit konnte eine deutlich schnellere Konvergenz erreicht werden, und auch die Abhängigkeit von der Stärke der Diffusion ist verringert. Bei der Berechnung der optimierten Koeffizienten wurde wie in Beispiel 8.3.1 mit dem Faktor 3 skaliert. 


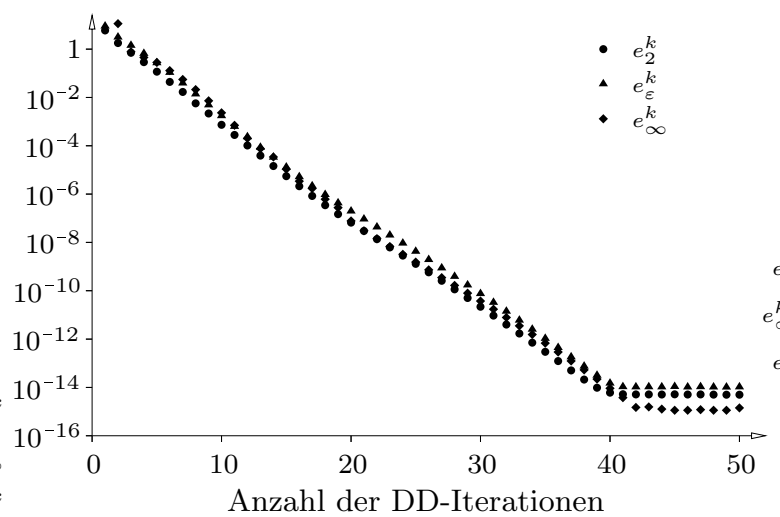

$\left(E_{10^{-4}}\right)$

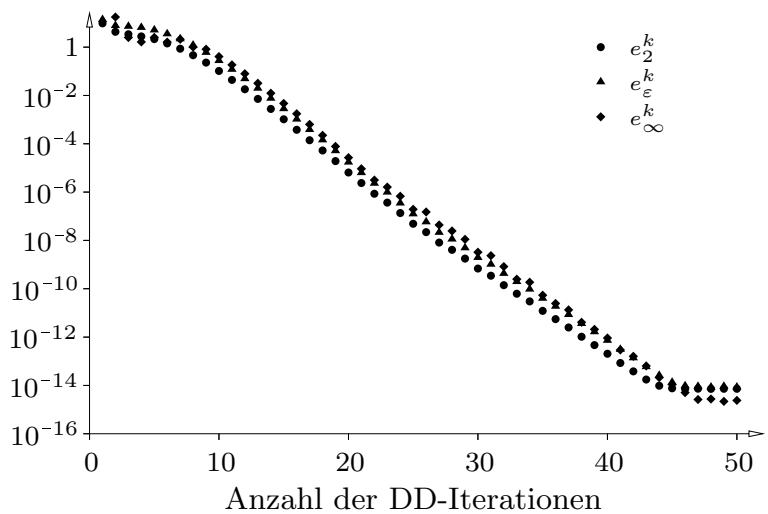

$\left(E_{10^{-6}}\right)$

Abbildung 8.8: Die Fehler $e_{2}^{k}, e_{\varepsilon}^{k}$ und $e_{\infty}^{k}$ in Abhängigkeit von der Anzahl der DD-Iterationen für die Fälle $\left(E_{10^{-4}}\right)$ und $\left(E_{10^{-6}}\right)$ bei punktweise optimiertem $\gamma$.

\subsection{Ausblick}

Ein Blick in die Literatur der letzten Jahre zeigt, daß Gebietszerlegungsalgorithmen für Evolutionsprobleme auf Grundlage der Waveform-Relaxation-Methode zu einem sehr aktivem Forschungfeld geworden sind. Insbesondere Verfahren, die mit Hilfe von optimierten SchwarzMethoden generierte, möglichst durchlässige Austausrandbedingungen nutzen, stehen im Zentrum des Interesses.

Im Rahmen der vorliegenden Arbeit wurde ein Dirichlet-Robin-Gebietszerlegungsverfahren vom Waveform-Relaxation-Typ eingeführt und für den Fall von zwei nicht überlappenden Teilgebieten untersucht. Zudem stellt diese Arbeit den Kontext für die Nutzung des Gebietszerlegungsverfahres im Zusammenhang mit einer Finite-Volumen-Diskretisierung bereit. Durch die Untersuchungen aus den Abschnitten 8.1 und 8.2 liegt für den betrachteten Algorithmus eine Strategie zur bezüglich der Konvergenzrate optimierten Wahl der Austauschrandbedingungen vor. Das Beispiel 8.3.4 demonstriert, wie sich die Strategie zur Optimierung der Austauschrandbedingungen auch in dem für Anwendungen relevanten Fall nicht konstanter Koeffizienten einsetzen läßt. Offen blieb die Frage nach der Ursache für den Skalierungsfaktor, der abhängig von der Stärke der Diffusion in unseren numerischen Resultaten auftrat. Diese Abweichung zwischen theoretischer Prognose und numerischen Resultaten bedarf weiterer Untersuchung.

Als ein Vorteil der Gebietszerlegungsverfahren vom Waveform-Relaxation-Typ („Gebietszerlegung vor Zeitdiskretisierung“") wurde bereits die Möglichkeit erwähnt, von Teilgebiet zu Teilgebiet unterschiedliche Zeitschrittweiten zu wählen. Diese Flexibilität in der Zeitdiskretisierung anhand von Testfällen zu demonstrieren, wird Gegenstand zukünftiger Arbeiten sein, auch vor dem Hintergrund nur weniger entsprechender Anwendungen in der Literatur.

Wir haben in dieser Arbeit ausschließlich polynomiale Approximationen des optimalen, globalen Randoperators $\Lambda$ von nullter Ordnung berücksichtigt, da man auf diese Weise gerade zu einer übliche Robin-Randbedingung am Austauschrand gelangt. Dies geschah im Einklang 
mit der Formulierung unseres Dirichlet-Robin-Gebietszerlegungsverfahrens und den anschließenden Konvergenzuntersuchungen aus Kapitel 6 bzw. Kapitel 7. Die Konvergenzraten, die in unseren numerischen Resultaten zu Tage traten, bleiben nicht hinter den Konvergenzraten zurück, die sich für zweidimensionale numerische Beispiele mit Approximation nullter Ordnung der optimalen Randbedingungen für beide Teilgebiete (also für Robin-Robin-Verfahren) in der Literatur finden (vergl. [49]). Vielfach erreichen wir sogar bessere Konvergenzraten. Die bezüglich der Konvergenzgeschwindigkeit überlegenen Verfahren für zweidimensionale Fälle werden in [49] stets mit einer Approximation von $\Lambda$ erster Ordnung erzielt. Eine entsprechende Erweiterung unseres Dirichlet-Robin-Verfahrens, also die Nutzung des Randoperators

$$
\Lambda_{1}=p+q \partial_{t}+q b_{2} \partial_{y}, \quad p, q \in \mathbb{R},
$$

(siehe Abschnitt 8.2) steht noch aus und erscheint uns vielversprechend.

Bei einem direkten Vergleich zwischen Dirichlet-Robin- und Robin-Robin-Algorithmen für elliptische Probleme in der Arbeit [2] liefert der Dirichlet-Robin-Algorithmus sehr gute Ergebnisse, speziell ohne zusätzlichen Austauschrandterm (d. h. $\gamma=0$ ). Damit entfällt dort eine Prozedur zur Optimierung der Austauschrandbedingungen ganz. Auch in unserem Fall ergab sich aus der Theorie, daß für gleichgewichtete Gebietsteilung und Relaxation mit $\sigma=\frac{1}{2}$ der Nulloperator optimaler Randoperator ist, somit explizit angegeben werden kann. Dieses Verhalten spiegelte sich jedoch in den numerischen Test nicht wieder, stattdessen stellten sich die besten Resultate für Werte von $\sigma$ etwas oberhalb von $\frac{1}{2}$ ein. Hier sehen wir ebenfalls weiteren Untersuchungsbedarf.

Insbesondere aus Sicht des Performance-Gewinns durch Parallelisierung der Rechnung ergibt sich der Bedarf nach einer Formulierung des Dirichlet-Robin-Algorithmus für mehr als zwei Teilgebiete. Liegt eine Zerlegung $\left\{\Omega_{1}, \ldots, \Omega_{\}}\right.$des Rechengebiets $\Omega$ vor mit einer Schwarz-Weiß-Einfärbung der Teilgebiete, so daß je zwei Teilgebiete $\Omega_{i}$ und $\Omega_{j}$ mit einer gemeinsamen Kante $\Gamma_{i j}$ von unterschiedlicher Färbung sind, so ergibt sich die Erweiterung des Dirichlet-Robin-Algorithmus unmittelbar. Auf allen Teilgebieten einer Färbung werden Dirichlet-, auf allen anderen Teilgebieten Robin-Probleme definiert. Liegt eine solche SchwarzWeiß-Einfärbung nicht vor, so können Teilgebiete auftreten, auf deren Austauschrändern abschnittsweise sowohl Dirichlet- als auch Robin-Bedingungen definiert werden. Der Algorithmus muß dann additiv formuliert werden. Von Interesse in diesem Zusammenhang ist die Frage nach dem Einfluß der Anzahl der Teilgebiete auf das Konvergenzverhalten. Auch dies betrifft zukünftige Untersuchungen. Zum Vergleich siehe Abschnitt 6.3 in [28], dort sind numerische Resultate für einen optimierten Robin-Robin-Algorithmus im Fall von mehr als zwei Teilgebieten zu finden. 


\section{Anhang}

\section{A.1 Notation}

Es bezeichne $\mathbb{N}_{0}$ die Menge der natürlichen Zahlen (einschließlich der 0 ). Soll die 0 ausgeschlossen sein, so wird dies durch $\mathbb{N}$ ausgedrückt. Jede natürliche Zahl $n$ wird auch als Menge $\{0, \ldots, n-1\}$ aufgefaßt. Für Abzählungen die bei 1 statt bei 0 beginnen, ist die Bezeichnung $\underline{n}=\{1, \ldots, n\}$ nützlich, dabei sei $\underline{n}=\emptyset$ für $n=0$. Dies erklärt kompakte Schreibweisen wie $i \in n$ oder $i \in \underline{n}$.

Ein System $\mathcal{Y}$ von Teilmengen einer Menge $X \subseteq \mathbb{R}^{d}$ heißt eine Zerlegung von $X$, falls gilt:

(Z1) $\bigcup \overline{\mathcal{Y}}=\bar{X}$

(Z2) $Y, Z \in \mathcal{Y}, Y^{\circ} \cap Z^{\circ} \neq \emptyset \Longrightarrow Y=Z$.

Für Mengen $A, B \subseteq \mathbb{R}^{d}$ schreiben wir abkürzend $B \Subset A$, falls $B$ präkompakt (d. h. beschränkt) ist und $\bar{B} \subseteq A$ gilt. $B^{A}$ steht für die Menge aller Abbildungen von einer Menge $A$ in eine Menge $B$.

Den Dualraum eines normierten Raumes $X$ bezeichnen wir mit $X^{*}$, das Dualitätsprodukt zwischen $X^{*}$ und $X$ mit $\langle\cdot, \cdot\rangle_{X^{*} \times X}$.

Darüber hinaus erinnern wir an die gebräuchliche Multiindexschreibweise. Für jedes $\alpha \in \mathbb{N}_{0}^{d}$ schreiben wir

$$
|\alpha|:=\sum_{i \in \underline{d}} \alpha_{i}, \quad D^{\alpha}:=\frac{\partial^{|\alpha|}}{\partial x_{1}^{\alpha_{1}} \cdots \partial x_{d}^{\alpha_{d}}}, \quad D^{j}:=D^{\alpha} \operatorname{mit} \alpha=\left(\delta_{i j}\right)_{i \in \underline{d}} .
$$




\section{A.2 Funktionenräume ortsabhängiger Funktionen}

\section{Lebesgue-Räume}

Es sei $\Omega$ ein Gebiet in $\mathbb{R}^{d}$. Dann bezeichnen wir mit $C(\Omega)$ die Menge aller stetigen reellwertigen Funktionen auf $\Omega$ und mit $C(\bar{\Omega})$ die Menge aller dieser Funktionen, die gleichmäßig stetig sind auf $\Omega$. Zu jedem $m \in \mathbb{N}_{0}$ definieren wir

$$
C^{m}(X):=\left\{u \in \mathbb{R}^{X} \mid \forall \alpha \in \mathbb{N}_{0}^{d} \text { mit }|\alpha| \leqslant m: D^{\alpha} u \in C(X)\right\}
$$

mit $X=\Omega$ oder $X=\bar{\Omega}$. Davon leiten wir die Bezeichnungen für glatte Funktionen und Testfunktionen

$$
C^{\infty}(X):=\bigcap_{m \in \mathbb{N}} C^{m}(X), \quad C_{0}^{\infty}(\Omega):=\left\{u \in C^{\infty}(\Omega) \mid \operatorname{supp}(u) \Subset \Omega\right\}
$$

ab, wobei die Menge $\operatorname{supp}(u):=\overline{\{x \in A \mid u(x) \neq 0\}}$ als Träger einer reellwertigen Funktion $u$ auf einer Menge $A$ bezeichnet wird.

Desweiteren geben wir die Standardräume Lebesgue-integrierbarer Funktionen wieder. Dazu sei $\mathbb{R}^{d}$ mit dem Lebesgue-Maß versehen. Für $p \in[1, \infty]$ sei dann

$$
L^{p}(\Omega):=\left\{v \in \mathbb{R}^{\Omega} \mid v \text { ist meßbar mit }\|v\|_{L^{p}(\Omega)}<\infty\right\}
$$

wobei

$$
\|v\|_{L^{p}(\Omega)}:= \begin{cases}\left(\int_{\Omega}|v|^{p} d t\right)^{\frac{1}{p}}, & p<\infty \\ \inf \left\{\sup _{x \in \Omega \backslash N}|v(x)| \mid N \subseteq \Omega \text { ist eine Nullmenge }\right\}, & p=\infty .\end{cases}
$$

Mit der Äquivalenzrelation $u \sim v: \Leftrightarrow\left(u=v\right.$ fast überall) wird die Quotientenmenge $L^{p}(\Omega) / \sim$ versehen mit $\|\cdot\|_{L^{p}(\Omega)}$ zu einem Banach-Raum (siehe [1], Theorem 2.10). Man schreibt für diesen wieder $L^{p}(\Omega)$. Speziell auf $L^{2}(\Omega)$ wird durch

$$
(u, v)_{L^{2}(\Omega)}:=\int_{\Omega} u v d x, \quad u, v \in L^{2}(\Omega),
$$

ein Skalarprodukt definiert, so daß $L^{2}(\Omega)$ mit diesem versehen ein Hilbert-Raum ist. Häufig schreiben wir einfach $(\cdot, \cdot)_{\Omega}$ oder $(\cdot, \cdot)$. Schließlich heißen Funktionen aus dem Raum

$$
L_{l o c}^{1}(\Omega):=\left\{v \in \mathbb{R}^{\Omega} \mid v \in L^{1}\left(\Omega^{\prime}\right) \text { für jedes Gebiet } \Omega^{\prime} \Subset \Omega\right\}
$$

lokal integrierbar über $\Omega$.

\section{Schwach differenzierbare Funktionen}

Die nachfolgende Definition der verallgemeinerten Ableitungen stellt eine Abschwächung des klassischen Differenzierbarkeitsbegriffs dar. Eine Funktion $v \in L_{l o c}^{1}(\Omega)$ besitzt eine verallgemeinerte (auch schwache) Ableitung zum Multiindex $\alpha \in \mathbb{N}_{0}^{d}$, falls ein $w \in L_{l o c}^{1}(\Omega)$ existiert mit 


$$
\int_{\Omega} v D^{\alpha} \varphi d x=(-1)^{|\alpha|} \int_{\Omega} w \varphi d x
$$

für alle $\varphi \in C_{0}^{\infty}(\Omega)$. Im Fall der Existenz ist $w$ in $L_{l o c}^{1}(\Omega)$ eindeutig bestimmt und wird mit $D^{\alpha} v$ bezeichnet. Es gilt $D^{0} v=v$. Als Sobolev-Räume der Funktionen mit verallgemeinerten Ableitungen definieren wir nun für $k \in \mathbb{N}_{0}$ und $p \in[1, \infty]$

$$
H^{k, p}(\Omega):=\left\{v \in L^{p}(\Omega) \mid \forall \alpha \in \mathbb{N}_{0}^{d} \text { mit }|\alpha| \leqslant k: D^{\alpha} u \in L^{p}(\Omega)\right\} .
$$

Versehen mit der Norm

$$
\|v\|_{k, p, \Omega}:= \begin{cases}\left(\sum_{|\alpha| \leqslant k}\left\|D^{\alpha} v\right\|_{L^{p}(\Omega)}^{p} d t\right)^{\frac{1}{p}}, & p<\infty \\ \max _{|\alpha| \leqslant k}\left\|D^{\alpha} v\right\|_{L^{\infty}(\Omega)}, & p=\infty\end{cases}
$$

ist $H^{k, p}(\Omega)$ ein Banach-Raum (siehe [1], Theorem 3.2), und speziell $H^{k}(\Omega):=H^{k, 2}(\Omega)$ wird daher mit dem Skalarprodukt

$$
(u, v)_{k, \Omega}:=\sum_{|\alpha| \leqslant k}\left(D^{\alpha} u, D^{\alpha} v\right)_{\Omega}, \quad u, v \in H^{k, 2}(\Omega),
$$

zu einem Hilbert-Raum. Wir schreiben kürzer $\|v\|_{k, \Omega}:=\|v\|_{k, 2, \Omega}$. Wegen $D^{0} v=v$ ist $\|v\|_{0, p, \Omega}=\|v\|_{L^{p}(\Omega)}$. Darüber hinaus nutzen wir die Halbnormen

$$
\begin{aligned}
|v|_{k, \Omega} & :=\left(\sum_{|\alpha|=k}\left\|D^{\alpha} v\right\|_{L^{2}(\Omega)}^{2} d t\right)^{\frac{1}{2}}, v \in H^{k}(\Omega), \\
|v|_{k, \infty, \Omega} & :=\max _{|\alpha|=k}\left\|D^{\alpha} v\right\|_{L^{\infty}(\Omega)} .
\end{aligned}
$$

Im Kontext geben wir das zugrundeliegende Gebiet im Index nicht an, schreiben also

$$
\|v\|_{k, p},\|v\|_{k},|v|_{k},\|v\|_{\infty} \quad \text { statt } \quad\|v\|_{k, p, \Omega},\|v\|_{k, \Omega},|v|_{k, \Omega},\|v\|_{L^{\infty}(\Omega)} .
$$

Schließlich bezeichne $H_{0}^{k}(\Omega)$ den Abschluß von $C_{0}^{\infty}(\Omega)$ bezüglich der Norm $\|\cdot\|_{k}$. Es ist $\left(H_{0}^{k}(\Omega),\|\cdot\|_{k}\right)$ ein Banach-Raum, da $H_{0}^{k}(\Omega)$ ein abgeschlossener Unterraum von $H^{k}(\Omega)$ ist.

Analog zu der verallgemeinerten Ableitung läßt sich die schwache Divergenz eines Vektorfeldes einführen. Eine Funktion $v \in\left(L_{l o c}^{1}(\Omega)\right)^{d}$ besitzt die schwache Divergenz $w \in L_{l o c}^{1}(\Omega)$, falls

$$
\int_{\Omega} v \cdot \nabla \varphi d x=-\int_{\Omega} w \varphi d x
$$

für alle $\varphi \in C_{0}^{\infty}(\Omega)$ gilt. Im Fall der Existenz schreiben wir $\nabla \cdot v$ für $w$. Man beachte, daß die schwache Divergenz $\nabla \cdot v$ existieren kann, selbst wenn nicht alle partiellen schwachen Ableitungen $D^{i} u, i \in \underline{d}$, existieren. Als Sobolev-Raum der Funktionen mit schwacher Divergenz definieren wir

$$
H(\operatorname{div} ; \Omega):=\left\{v \in\left(L^{2}(\Omega)\right)^{d} \mid \nabla \cdot v \in L^{2}(\Omega)\right\} .
$$

Für Funktionen, deren schwache Divergenz sogar wesentlich beschränkt bleibt, gilt die verallgemeinerte Produktregel. 
Satz A.2.1 Sei $u \in H^{1}(\Omega)$ und $v \in\left(L_{l o c}^{1}(\Omega)\right)^{d}$ ein Vektorfeld mit schwacher Divergenz $\nabla \cdot v \in L^{\infty}(\Omega)$. Dann existiert auch $\nabla \cdot(u v) \in H(\operatorname{div} ; \Omega)$ und es gilt

$$
\nabla \cdot(u v)=u \nabla \cdot v+v \cdot \nabla u \text {. }
$$

Beweis. $\quad$ Siehe [9], Lemma 1.1.40. 
Unter einer ganzen Reihe von Einbettungssätzen zitieren wir

Satz A.2.2 Ist $\Omega \subseteq \mathbb{R}^{2}$ ein polygonales Gebiet, so existiert die stetige Einbettung

$$
H^{1, p}(\Omega) \hookrightarrow C(\Omega)
$$

für $p>2$.

Satz A.2.2 läßt sich allgemeiner formulieren für Gebiete $\Omega \subseteq \mathbb{R}^{d}$, die die sogenannte Kegeleigenschaft besitzen (siehe [1], Theorem 5.4).

\section{Randwerte, Spursätze}

Unser nächstes Ziel ist es, Randwerte für Funktionen aus $H^{1}(\Omega)$ und $H$ (div; $\Omega$ ) zu definieren. Dazu setzen wir voraus, daß $\Omega$ einen Lipschitz-stetigen Rand besitzt. Es sei $\Gamma$ eine nicht leere, relativ offene, zusammenhängende Teilmenge von $\partial \Omega$. Wir definieren den Sobolev-Raum

$$
\begin{aligned}
H^{\frac{1}{2}}(\Gamma):=\left\{v \in L^{2}(\Gamma) \mid\|v\|_{H^{\frac{1}{2}(\Gamma)}}<\infty\right\} \\
\operatorname{mit}\|v\|_{H^{\frac{1}{2}(\Gamma)}}^{2}:=\langle v, v\rangle_{H^{\frac{1}{2}}(\Gamma)} \text { und } \\
\langle u, v\rangle_{H^{\frac{1}{2}(\Gamma)}}:=\int_{\Gamma} u v d s+\iint_{\Gamma \times \Gamma} \frac{(u(x)-u(y))(v(x)-v(y))}{|x-y|^{d}} d s(x, y), \quad u, v \in H^{\frac{1}{2}}(\Gamma) .
\end{aligned}
$$

$H^{\frac{1}{2}}(\Gamma)$ bildet mit dem Skalarprodukt $\langle\cdot, \cdot\rangle_{H^{\frac{1}{2}}(\Gamma)}$ einen Hilbert-Raum (siehe [56], Abschnitt 1.2.3). Wir greifen zudem auf einen Teilraum von $H^{\frac{1}{2}}(\Gamma)$ zurück, nämlich

$$
H_{00}^{\frac{1}{2}}(\Gamma):=\left\{v \in L^{2}(\Gamma) \mid v^{\circ} \in H^{\frac{1}{2}}(\partial \Omega)\right\},
$$

wobei $w^{\circ}$ für jede Funktion $w$ auf $\Gamma$ deren Fortsetzung auf $\partial \Omega$ durch Null darstellt. Durch das Skalarprodukt

$$
\langle u, v\rangle_{H_{00}^{\frac{1}{2}}(\Gamma)}:=\langle u, v\rangle_{H^{\frac{1}{2}}(\Gamma)}+\int_{\Gamma} \frac{u v}{\operatorname{dist}(x, \partial \Gamma)} d s, \quad u, v \in H_{00}^{\frac{1}{2}}(\Gamma)
$$

wird $H_{00}^{\frac{1}{2}}(\Gamma)$ zum Hilbert-Raum mit der Norm $\|u\|_{H_{00}^{\frac{1}{2}}(\Gamma)}^{2}:=\langle v, v\rangle_{H_{00}^{\frac{1}{2}}(\Gamma)}$, die äquivalent ist zur Norm $\left\|v^{\circ}\right\|_{H^{\frac{1}{2}}(\Gamma)}$ (siehe [66], Abschnitt 4.1). In der Definition von $\langle\cdot, \cdot\rangle_{H_{00}^{\frac{1}{2}}(\Gamma)}$ bezeichne $\operatorname{dist}(x, \partial \Gamma)$ den Abstand von $x \in \Gamma$ zu $\partial \Gamma$ ( $\partial \Gamma$ Rand von $\Gamma$ bezüglich der Relativtopologie auf $\partial \Omega) \operatorname{mit} \operatorname{dist}(x, \emptyset):=\infty$. Falls $\Gamma$ eine Zusammenhangskomponente von $\partial \Omega$ ist, so folgt $\partial \Gamma=\emptyset$, in diesem Falle stimmen die Räume $H^{\frac{1}{2}}(\Gamma)$ und $H_{00}^{\frac{1}{2}}(\Gamma)$ überein.

Durch Fortsetzung des Spuroperators für stetige Funktionen werden nun Randwerte für Funktionen aus $H^{1}(\Omega)$ erklärt. 
Satz A.2.3 Der Spuroperator

$$
\left.u \mapsto u\right|_{\Gamma}, \quad u \in C^{\infty}(\bar{\Omega})
$$

kann eindeutig fortgesetzt werden zu einem linearen und stetigen Operator

$$
\operatorname{tr}_{\Gamma}: H^{1}(\Omega) \rightarrow H^{\frac{1}{2}}(\Gamma),
$$

ebenfalls Spuroperator genannt. $Z u \operatorname{tr}_{\Gamma}$ existiert eine lineare und stetige Rechtsinverse $\operatorname{tr}_{\Gamma}{ }^{-}$, d. h. $\operatorname{tr}_{\Gamma} \operatorname{otr}_{\Gamma}^{-}=\mathrm{id}_{H^{\frac{1}{2}}(\Gamma)}$.

Beweis. $\quad$ Siehe [52], Theorem 3.37.

$\operatorname{tr}_{\Gamma} u$ wird als Randwerte von $u$ (auf $\Gamma$ ) im Sinne der Spur bezeichnet. Gelegentlich schreiben wir $\left.u\right|_{\Gamma}$ statt $\operatorname{tr}_{\Gamma} u$ auch für Funktionen $u \in H^{1}(\Omega)$.

Das entsprechende Resultat für das Produkt einer Funktion $u \in H(\operatorname{div} ; \Omega)$ mit der äußeren Normale („Fluß von $u$ durch Berandung von $\left.\Omega^{\prime \prime}\right)$ lautet

Satz A.2.4 Es sei $\Omega$ ein beschränktes Lipschitz-Gebiet. Dann kann der NormalenSpuroperator

$$
\left.u \mapsto u\right|_{\partial \Omega} \cdot n, \quad u \in\left(C^{\infty}(\bar{\Omega})\right)^{d}
$$

zur äußeren Normale $n$ von $\partial \Omega$ eindeutig fortgesetzt werden zu einem linearen und stetigen Operator

$$
\operatorname{ntr}_{\partial \Omega}: H(\operatorname{div} ; \Omega) \rightarrow\left(H^{\frac{1}{2}}(\partial \Omega)\right)^{*}
$$

ebenfalls Normalen-Spuroperator genannt. $\mathrm{ntr} \partial \Omega$ besitzt eine lineare und stetige Rechtsinverse $\operatorname{ntr}_{\partial \Omega^{-}}$.

Beweis. [32], Seite 27.

Folgerung A.2.5 Es sei $\Omega$ ein beschränktes Lipschitz-Gebiet. Für Funktionen $(u, v) \in H(\operatorname{div} ; \Omega) \times H^{1}(\Omega)$ gilt die (verallgemeinerte) Greensche Formel

$$
\int_{\Omega} v \nabla \cdot u d x+\int_{\Omega} u \cdot \nabla v d x=\left\langle\operatorname{ntr}_{\partial \Omega} u, \operatorname{tr}_{\partial \Omega} v\right\rangle .
$$

Satz A.2.6 Es sei $\Omega \subset \mathbb{R}^{d}$ ein beschränktes Lipschitz-Gebiet und $\Gamma$ eine nicht leere, relativ offene, zusammenhängende Teilmenge von $\partial \Omega$. Dann gilt für alle $v \in H^{1}(\Omega) \operatorname{mit} \operatorname{tr}_{\Gamma} v=0$ die Ungleichung

$$
\|v\|_{0} \leqslant C|v|_{1}
$$

mit einer Konstante $C$, die allein von $\Omega$ und $\Gamma$ abhängt.

Beweis. $\quad$ Siehe [57], Theorem 1.3.3. 


\section{A.3 Funktionenräume zeitabhängiger Funktionen}

\section{Lebesgue-Räume vektorwertiger Funktionen}

Zur Behandlung zeitabängiger Probleme geben wir hier die gängige Verfahrensweise wieder, bei der Funktionen über einem Raum-Zeit-Zylinder $(0, T) \times \Omega$ aufgefaßt werden als vektorwertige Funktionen $(0, T) \rightarrow V$ mit einem geeigneten Banach-Raum $\left(V,\|\cdot\|_{V}\right)$. Der Ortsund Zeitabhängigkeit werden also unterschiedlich Rechnung getragen. In diesem Zusammenhang bedient man sich eines speziellen Integralbegriffs, des Bochner-Integrals $\int_{a}^{b} v(t) d t$ für meßbare Funktionen $v \in V^{(a, b)}$ (für Details siehe [65], 24). Dessen Existenz läßt sich auf die Existenz eines Lebesgue-Integrals zurückführen, denn für meßbare Funktionen $v \in V^{(a, b)}$ existiert $\int_{a}^{b} v(t) d t$ genau dann, wenn $\int_{a}^{b}\|v(t)\|_{V} d t$ existiert.

Für $p \in[1, \infty]$ definieren wir die Lebesgue-Räume vektorwertiger Funktionen

$$
L^{p}(0, T ; V):=\left\{v \in V^{(0, T)} \mid v \text { ist meßbar mit }\|v\|_{L^{p}(0, T ; V)}<\infty\right\}
$$

wobei

$$
\|v\|_{L^{p}(0, T ; V)}:= \begin{cases}\left(\int_{0}^{T}\|v(t)\|_{V}^{p} d t\right)^{\frac{1}{p}}, & p<\infty \\ \inf \left\{C>0 \mid\|v(t)\|_{V}<C \text { für fast alle } t \in(0, T)\right\}, & p=\infty\end{cases}
$$

Identifiziert man Funktionen, die sich lediglich auf einer Menge vom Lebesgue-Maß Null unterscheiden, so wird $L^{p}(0, T ; V)$ versehen mit $\|\cdot\|_{L^{p}(0, T ; V)}$ zum Banach-Raum. Ist $\left(V,(\cdot, \cdot)_{V}\right)$ ein Hilbert-Raum, so wird speziell $L^{2}(0, T ; V)$ mit dem Skalarprodukt

$$
(u, v)_{L^{2}(0, T ; V)}:=\int_{0}^{T}(u(t), v(t))_{V} d t, \quad u, v \in L^{2}(0, T ; V),
$$

ebenfalls zu einem Hilbert-Raum (siehe [67], Proposition 23.2).

Für den Dualraum von $L^{p}(0, T ; V)$ ergibt sich der folgende Zusammenhang:

Lemma A.3.1 Es sei $(V,\|\cdot\|)$ ein Banach-Raum. Dann gilt

$$
L^{p}(0, T ; V)^{*}=L^{q}\left(0, T ; V^{*}\right)
$$

für alle $p, q \in(1, \infty)$ mit $\frac{1}{p}+\frac{1}{q}=1$.

Beweis. $\quad$ Siehe [67], Proposition 23.7.

\section{Funktionen mit schwacher Zeitableitung}

Die klassische partielle Integration für Funktionen ist Vorlage für den Begriff der verallgemeinerten Zeitableitung. Sind $V$ und $W$ Banach-Räume und ist $v \in L^{1}(0, T ; V)$, so heißt $w \in L^{1}(0, T ; W)$ n-te verallgemeinerte (zeitliche) Ableitung von $v$, falls 


$$
\int_{0}^{T} \varphi^{(n)}(t) v(t) d t=(-1)^{n} \int_{0}^{T} \varphi(t) w(t) d t
$$

für alle $\varphi \in C_{0}^{\infty}(0, T)$. Für $n=1$ bezeichnen wir gegebenenfalls $w$ mit $v^{\prime}$. Verallgemeinerte Zeitbleitung lassen sich auch mit Hilfe von verktorwertigen Distributionen formulieren, denn nach der obigen Definition ist $w$ die Distributionsableitung von $u$ (siehe [67], Remark 23.17).

Mit dem nächsten Satz wird die Existenz und Eindeutigkeit von verallgemeinerten Zeitableitungen im Fall von Evolutionsgleichungen sichergestellt. Es sei $U$ ein reeller, separabler Hilbert-Raum und $V$ ein reeller, reflexiver Banach-Raum, stetig und dicht in $U$ eingebettet. Dann bezeichnet man das Tripel $\left(V, U, V^{*}\right)$ als Evolutionstripel (auch Gelfand-Dreier). Für die Abbildung $\Phi: U \ni u \mapsto f_{u}$ mit $f_{u}: V \ni v \mapsto(u, v)_{U}$ gilt dann $\Phi[U] \subseteq V^{*}$. Ferner ist $\Phi$ injektiv, stetig und linear, daher identifiziert man $U$ mit $\Phi[U]$ und schreibt abkürzend $V \subseteq U \subseteq V^{*}$

Satz A.3.2 Es sei $V \subseteq U \subseteq V^{*}$ ein Evolutionstripel, und es seien $p, q \in[1, \infty]$. Für jedes $u \in L^{p}(0, T ; V)$ ist dann $u^{\prime} \in L^{q}\left(0, T ; V^{*}\right)$ eindeutig bestimmt, und es gilt

$$
\frac{d}{d t}(u(t), v)_{U}=\left\langle u^{\prime}(t), v\right\rangle_{V^{*} \times V}
$$

für fast alle $t \in(0, T)$ und alle $v \in V$.

Beweis. $\quad$ Siehe [67], Proposition 23.20.

Für $p, q \in(1, \infty)$ mit $\frac{1}{p}+\frac{1}{q}=1$ definieren wir die Sobolev-Räume vektorwertiger Funktionen

$$
W^{1, p}(0, T ; V):=\left\{v \in L^{p}(0, T ; V) \mid v^{\prime} \in L^{q}\left(0, T ; V^{*}\right)\right\} .
$$

Ist $V \subseteq U \subseteq V^{*}$ ein Evolutionstripel, dann bildet $W^{1, p}(0, T ; V)$ mit der Norm

$$
\|v\|_{W^{1, p}(0, T ; V)}:=\|v\|_{L^{p}(0, T ; V)}+\left\|v^{\prime}\right\|_{L^{q}\left(0, T ; V^{*}\right)}
$$

einen Banach-Raum (siehe [67], Proposition 23.23). Speziell für $p=2$ schreiben wir $H^{1}(0, T ; V):=W^{1,2}(0, T ; V)$.

Der nachfolgende Satz stellt klar, in welchem Sinne Anfangsbedingungen $u(0)=u_{0}$ für Funktionen $u \in W^{1, p}(0, T ; V)$ zu verstehen sind.

Satz A.3.3 Es sei $V \subseteq U \subseteq V^{*}$ ein Evolutionstripel, und es seien $p, q \in(1, \infty)$ mit $\frac{1}{p}+\frac{1}{q}=1$. Dann ist $W^{1, p}(0, T ; V)$ stetig eingebettet in den Raum $C([0, T] ; U)$ der stetigen Abbildungen aus $U^{[0, T]}$. Es gibt also zu jedem $u \in W^{1, p}(0, T ; V)$ ein eindeutig bestimmtes $v \in C([0, T] ; U)$, das fast überall in $[0, T]$ mit u übereinstimmt.

Beweis. $\quad$ Siehe [67], Proposition 23.23.

Für Funktionen mit verallgemeinerter Zeitableitung besteht die folgende Regel zur partiellen Integration. 
Satz A.3.4 Es sei $V \subseteq U \subseteq V^{*}$ ein Evolutionstripel, und es seien $p, q \in(1, \infty)$ mit $\frac{1}{p}+\frac{1}{q}=1$. Dann gilt für alle $u, v \in W^{1, p}(0, T ; V)$ und $0 \leqslant s \leqslant t \leqslant T$

$$
(u(t), v(t))_{U}-(u(s), u(s))_{U}=\int_{s}^{t}\left\langle u^{\prime}(\tau), v(\tau)\right\rangle_{V^{*} \times V}+\left\langle v^{\prime}(\tau), u(\tau)\right\rangle_{V^{*} \times V} d \tau .
$$

Beweis. $\quad$ Siehe [67], Proposition 23.23.

Wie in der klassischen Analysis zeigt man damit und per Induktion eine Darstellung durch Taylorpolynom und Integralrestglied für hinreichend differenzierbare Funktionen $u$ :

$$
u(t)=\sum_{i=0}^{n} \frac{u^{(i)}(s)}{i !}(t-s)^{i}+\frac{1}{n !} \int_{s}^{t}(t-\tau)^{n} u^{(n)}(\tau) d \tau
$$

\section{Sobolev-Räume gebrochener Ordnung}

Abschließend führen wir noch die folgenden Sobolev-Räume gebrochener Ordnung ein. Für $s \in(0,1)$ sei

$$
H^{s}\left(0, T ; L^{2}(\Gamma)\right):=\left\{v \in L^{2}(\Gamma)^{(0, T)} \mid\|v\|_{H^{s}\left(0, T ; L^{2}(\Gamma)\right)}<\infty\right\}
$$

mit

$$
\|v\|_{H^{s}\left(0, T ; L^{2}(\Gamma)\right)}:=\left(\int_{0}^{T}\|v\|_{L^{2}(\Gamma)}^{2} d t+\int_{0}^{T} \int_{0}^{T} \frac{\|v(t)-v(\tau)\|_{L^{2}(\Gamma)}^{2}}{|t-\tau|^{d+2 s}} d \tau d t\right)^{\frac{1}{2}}
$$

(vergl. [12], Abschnitt 2.10). Man beachte, daß der Raum $H^{s}\left(0, T ; L^{2}(\Gamma)\right)$ auch per Interpolation $\left[H^{m}\left(0, T ; L^{2}(\Gamma)\right), H^{0}\left(0, T ; L^{2}(\Gamma)\right)\right]_{\theta},(1-\theta) m=s$, definiert werden kann, wie dies durchweg in [45] geschieht. Zur Gleichwertigkeit beider Definitionen siehe [63], Abschnitte 1.2.4 und 1.6.4.

Speziell greifen wir auf den Raum $H^{\frac{1}{4}}\left(0, T ; L^{2}(\Gamma)\right)$ zurück und definieren

$$
Z:=Z(0, T ; \Gamma):=L^{2}\left(0, T ; H^{\frac{1}{2}}(\Gamma)\right) \cap H^{\frac{1}{4}}\left(0, T ; L^{2}(\Gamma)\right) .
$$

Versehen mit dem Skalarprodukt

$$
\langle v(t), w(t)\rangle_{Z}:=\int_{0}^{T}\langle v(t), w(t)\rangle_{H^{\frac{1}{2}(\Gamma)}}+\int_{0}^{T} \frac{\langle v(t)-v(\tau), w(t)-w(\tau)\rangle_{L^{2}(\Gamma)}}{|t-\tau|^{\frac{3}{2}}} d \tau d t, \quad v, w \in Z,
$$

ist $Z$ ein Hilbert-Raum, siehe [45], Seiten 6 und 8 . 


\section{Literaturverzeichnis}

[1] R. A. Adams Sobolev Spaces, Academic Press Inc., New York, 1975.

[2] A. Alonso, R. L. Trotta and A. Valli Coercive domain decomposition algorithms for advection-diffusion equations and systems, J. Comput. Appl. Math. 96 (1998), 51-76.

[3] L. Angermann Numerical solution of second-order elliptic equations on plane domains, $\mathrm{M}^{2}$ An 25 (1991) 169-191.

[4] L. Angermann Error estimates for the finite-element solution of an elliptic singularly perturbed problem, IMA J. Numer. Anal. 15 (1995), 161-196.

[5] L. Angermann An upwind scheme of finite volume type with reduced crosswind diffusion, Bericht 165, Universität Erlangen-Nürnberg (1995).

[6] L. Angermann Skript zu Finite-Volumen-Methoden (2000).

[7] R. E. BANK and D. J. Rose Some error estimates for the box method, SIAM J. Numer. Anal. 24 (1987), 777-787.

[8] T. J. Barth and M. Ohlberger Finite Volume Methods: Foundation and Analysis, in: Encyclopedia of Computational Mechanics, Volume 1, Fundamentals, John Wiley and Sons, Ltd, 2004.

[9] J. Bey Finite-Volumen- und Mehrgitter-Verfahren für elliptische Randwertprobleme, PhD thesis, Eberhard-Karls-Universität, Tübingen, 1997.

[10] M. BJøRHUs A note on the convergence of discretized dynamic iteration, BIT 35 (1995), 291-296.

[11] P. E. BJøRstad and O. B. Widlund Iterative methods for the solution of elliptic problems on regions partitioned into substructures, SIAM J. Numer. Anal., 23 (1986), 1097-1120.

[12] F. Brezzi and G. Gilardi Functional Spaces, in: H. Kardestuncer and D. H. Norrie (Eds.), Finite Element Handbook McGraw-Hill Publ. , New York, 1987.

[13] X.-C. CAI Additive Schwarz algorithms for parabolic convection-diffusion equations, Numer. Math. 60 (1991), 41-61.

[14] X.-C. CAI Multiplicative Schwarz methods for parabolic problems, SIAM J. Sci. Comput. 15 (1994), 587-603. 
[15] Z. CAI On the finite volume element method, Numer. Math. 58 (1991), 713-735.

[16] P. Charton, F. Nataf and F. Rogier Méthode de décomposition e domaine pour l'équation d'advection-diffusion, C. R. Acad. Sci. 313(9) (1991), 623-626.

[17] Ph. G. Ciarlet Basic error estimates for elliptic problems, in: Ph. G. Ciarlet and J.L. Lions (Eds.), Handbook of Numerical Analysis II, North-Holland, Amsterdam, 1991.

[18] M. Dryja Substructuring methods for parabolic problems, in: R. Glowinski, Y. A. Kuznetsow, G. A. Meurant, J. Périaux, O. Widlund (Eds.), Fourth International Symposium on Doamin Decompostion Methods for Partial Differential Equations, SIAM, Philadelphia (1991).

[19] B. Engquist and A. MAJdA Absorbing boundary conditions for the numerical simulation of waves, Math. Comp. 31 (1977), 629-651.

[20] B. Engquist and H.-K. Zhao Absorbing boundary conditions for domain decomposition, Appl. Numer. Math. 27 (1998), 341-365.

[21] R. Eymard, T. Gallouet and R. Herbin The finite volume method, in: Ph. Ciarlet J. L. Lions (Eds.), Handbook for Numerical Analysis, North Holland, 2000, 715-1022.

[22] M. Feistauer, J. Felcman and M. Lukáčová-Medvid'ová On the convergence of a combined finite volume - finite element method for nonlinear convection - diffusion problems. Num. Methods for Part. Diff. Eqs., 13 (1997), 163-190.

[23] M. Feistauer, J. Felcman, M. Lukáčová-Medvid’ová and G. Warnecke Error estimates for a combined finite volume-finite element method for nonlinear convectiondiffusion problems, SIAM J. Numer. Anal. 36(5) (1999), 1528-1548.

[24] H. Fujita and T. Suzuki Evolution Problems in: P. G. Ciarlet and J. L. Lions (Eds.) Handbook of Numerical Analysis, Vol. II North-Holland, Amsterdam, 1991.

[25] M. J. GAnder Overlapping Schwarz waveform relaxation for parabolic problems, in: J. Mandel, C. Farhat, X.-C. Cai (Eds.), Tenth International Conference on Domain Decomposition Methods, AMS, Contemporary Mathematics 218 (1998).

[26] M. J. Gander, L. Halpern and F. Nataf Optimal convergence for overlapping an non-overlapping Schwarz waveform relaxation, in: C.-H. Lai, P. Bjøstad, M. Cross, O. Widlund (Eds.), Eleventh International Conference on Domain Decomposition Methods (1999), 27-36.

[27] M. J. GAnder and L. HALPERn Absorbing boundary conditions for the wave equation and parallel computing, Math. Comp. 74 (2005), 153-176.

[28] M. J. Gander and L. Halpern Optimized Schwarz Waveform Relaxation Methods for Advection Reaction Diffusion Problems, eingereicht.

[29] M. J. Gander, L. Halpern and M. Kern A Schwarz-Waveform Relaxation Method for Advection-Diffusion-Reaction Problems with Discontinuous Coefficients and non-Matching Grids, Proc. 16. Conf. Domain Decomposition Methods 2005, eingereicht. 
[30] M. J. GANDER and A. M. Stuart Space-time continuous analysis of waveform relaxation for the heat equation, SIAM J. Sci. Comput. 19 (1998), 2014-2031.

[31] E. Giladi and H. B. Keller Space-time domain decomposition for parabolic problems, Numerische Mathematik 93 (2002), 279-313.

[32] V. Girault and P.-A. Raviart Finite Element Methods for Navier-Stokes Equations, vol. 5 of Springer Series in Computational Mathematics, Springer-Verlag, Berlin, 1986.

[33] P. Grisvard Behaviour of the solutions of an elliptic boundary value problem in a polygonal or polyhedral domain, in: Numerical Solution of Partial Differential Equations, III, B. Hubbard (Eds.), Academic Press, New York, 1976.

[34] P. Grisvard Elliptic problems in nonsmooth domains, Pitman, Marshfield, 1985.

[35] W. HackBusch On first and second order box schemes, Computing 41 (1989), 277-296.

[36] L. Halpern Artificial Boundary Conditions for the Advection-Diffusion Equations, Math. Comp. vol 174 (1986), 425-438.

[37] R. Hangleiter and G. Lube Stabilized Galerkin methods and layer-adapted grids for elliptic problems, Comput. Methods Appl. Mech. Engrg. 166 (1998), 165-182.

[38] C. Hirsch Numerical Computation of Internal and External Flows, Vol. I: Fundamentals of Numerical Discretization. Wiley, New York, 1988.

[39] C. Japhet, F. Nataf and F. Rogier The optimized Order 2 Method. Application to Convection-Diffusion Problems, Future Generation Computer Systems 18 (2000).

[40] R. Jeltsch and B. Prohl Waveform relaxation with overlapping splittings, SIAM J. Sci. Comput. 19 (1995), 2014-2031.

[41] P. Knabner und L. Angermann Numerical Methods for Elliptic and Parabolic Partial Differential Equations Springer-Verlag, New York, 2003.

[42] O. A. Ladyženskaja, V. A. Solonnikov and N. N. Ural'Ceva Linear and Quasilinear Equations of Parabolic Type, AMS Translations of Mathematical Monograhps, Vol. 23 (1968).

[43] R. D. Lazarov, I. D. Mishev and P. S. Vassilevski Finite volume methods for convection-diffusion problems, SIAM J. Numer. Anal. 33 (1996), 31-55.

[44] E. Lelarasmee, A. E. Ruehli and A. L. Sangiovanni-Vincentelli The waveform relaxation method for time-domain analysis of large scale integrated circuits, IEEE Trans. on CAD of IC and Syst. 1 (1982), 131-145.

[45] J. L. Lions and E. Magenes Non-Homogeneous Boundary Value Problems and Applications, Vol. II Springer-Verlag, Berlin, 1972.

[46] G. Lube, F. C. Oтto and L. Müller A non-overlapping domain decomposition method for parabolic initial-boundary value problems, Applied Numerical Mathematics 28 (1998), 359-369. 
[47] G. LubE Numerik elliptischer Randwertprobleme, Vorlesung Wintersemester 2001/2002, Göttingen.

[48] V. MARTin An optimized Schwarz waveform relaxation method for the unsteady convection diffusion equation, Comput. Fluids 33 (2004), 829-837.

[49] V. Martin An optimized Schwarz waveform relaxation method for the unsteady convection diffusion equation, Applied Numerical Mathematics 52 (2005), 401-428.

[50] R. W. MacCormack and A. J. Paullay Computational efficiency achieved by time splitting of finite difference operators AIAA paper 72-154 (1972).

[51] P. W. MCDonald The computation of transonic flow through two-dimensional gas turbine cascades, ASME Paper 71-GT-89 (1971).

[52] W. McLean Strongly Elliptic Systems and Boundary Integral Equations University Press, Cambridge, 2000.

[53] U. MiekKala and O. Nevanlinna Convergence of dynamic iteration methods for initial value problems, SIAM J. Sci. Stat. Computing 8 (1987), 459-482.

[54] F. NAtaF and F. Rogier Factorization of the convection-diffusion operator and the Schwarz algorithm, $\mathrm{M}^{3}$ AS 5(1) (1995), 67-93.

[55] O. Nevanlinna Remarks on Picard-Lindelöf iterations, BIT 29 (1989), 328-346, 535562 .

[56] S. Nicaise Polygonal Interface Problems, Methoden und Verfahren der mathematischen Physik Band 39 Peter Lang, Frankfurt am Main, 1993.

[57] A. Quarteroni and A. Valli Numerical Approximation of Partial Differential Equations Springer-Verlag, Berlin, 1997.

[58] A. Quarteroni and A. Valli Domain Decomposition Methods for Partial Differential Equations Oxford University Press, Oxford, New York, 1999.

[59] H.-G. Roos, M. Stynes und L. Tobiska Numerical Methods for Singularly Perturbed Differential Equations Springer-Verlag, Berlin, 1996.

[60] T. SkalickÝ Vergleich einer Finite-Element- und einer Finite-Volumen-Methode: I. skalares Konvektions-Diffusions-Problem, Bericht B 891 Technische Universität Dresden (1996).

[61] V. Thомeе Galerkin Finite Element Methods for Parabolic Problems Springer-Verlag, Berlin, 1997.

[62] A. Toselli and O. B. Widlund Domain decomposition methods-algorithms and theory, Springer, New York, 2005.

[63] H. Triebel Theory of Function Spaces II, Birkhäuser, Basel, 1992. 
[64] O. B. Widlund Domain Decomposition Algorithms and the Bicentennial of the French Revolution, , in: T.-F. Chan, R. Glowinski, J. Périaux, and O. B. Widlund (Eds.), Third International Symposium on Domain Decomposition Methods for Partial Differential Equations, SIAM, Philadelphia (1990), xv-xx.

[65] J. Wloka Partielle Differentialgleichungen, Teubner Verlagsanstalt, Leipzig, 1982.

[66] J. XU and J. Zou Some nonoverlapping domain decomposition methods, SIAM Rev. 40 (1998), $857-914$.

[67] E. ZEIDLER Nonlinear Functional Analysis and its Applications II/A: Linear Monotone Operators, Springer-Verlag, New York (1990). 